\title{
Aluminum nitride deposition/characterization \& pMEMs/SAW device simulation/fabrication
}

Vincent Richard Pagan

West Virginia University

Follow this and additional works at: https://researchrepository.wvu.edu/etd

\section{Recommended Citation}

Pagan, Vincent Richard, "Aluminum nitride deposition/characterization \& pMEMs/SAW device simulation/ fabrication" (2009). Graduate Theses, Dissertations, and Problem Reports. 2012.

https://researchrepository.wvu.edu/etd/2012

This Thesis is protected by copyright and/or related rights. It has been brought to you by the The Research Repository @ WVU with permission from the rights-holder(s). You are free to use this Thesis in any way that is permitted by the copyright and related rights legislation that applies to your use. For other uses you must obtain permission from the rights-holder(s) directly, unless additional rights are indicated by a Creative Commons license in the record and/ or on the work itself. This Thesis has been accepted for inclusion in WVU Graduate Theses, Dissertations, and Problem Reports collection by an authorized administrator of The Research Repository @ WVU. For more information, please contact researchrepository@mail.wvu.edu. 
ALUMINUM NITRIDE DEPOSITION / CHARACTERIZATION \& PMEMS / SAW DEVICE SIMULATION / FABRICATION

\author{
by \\ Vincent Richard Pagán \\ Thesis submitted to the College of Engineering and Mineral Resources \\ at West Virginia University \\ in partial fulfillment of the requirements \\ for the degree of \\ Master of Science \\ in \\ Electrical Engineering
}

\author{
Approved by \\ Dimitris Korakakis, Ph.D., Committee Chairperson \\ Parviz Famouri, Ph.D. \\ Lawrence A. Hornak, Ph.D. \\ Osama M. Mukdadi, Ph.D.
}

Lane Department of Computer Science and Electrical Engineering

$$
\text { Morgantown, West Virginia }
$$

2009

Keywords: Aluminum Nitride, Microelectromechanical Systems, Surface Acoustic Wave, Finite Element Analysis

Copyright 2009 Vincent R. Pagán 


\section{Abstract \\ ALUMINUM NITRIDE DEPOSITION / CHARACTERIZATION \& PMEMS / SAW DEVICE SIMULATION / FABRICATION}

\section{by Vincent R. Pagán}

Aluminum Nitride (AIN) is a promising material for piezoelectric MicroElectroMechanical Systems (pMEMS) and Surface Acoustic Wave (SAW) devices. AlN is a direct bandgap semiconductor possessing moderate piezoelectric coefficients, a high Curie temperature, and a high acoustic velocity. Potential applications of AIN thin film devices include high temperature pMEMS microvalves for use in Solid Oxide Fuel Cell (SOFC) flow control systems and high frequency/sensitivity SAW platforms for use in biosensors.

Since AlN is a robust material capable of operating at high temperatures and harsh environments, it can be used in settings where other widely used piezoelectrics such as Lead Zirconate Titanate (PZT) and Zinc Oxide $(\mathrm{ZnO})$ fail. Piezoelectric beams are commonly used in MEMS and have many possible applications in smart sensor and actuator systems. In this work, the results of 3-dimensional Finite Element Analysis (FEA) of AlN homogeneous bimorphs ( $d_{31}$ mode) are shown. The coupled-field FEA simulations were performed using the commercially available software tool ANSYS $^{\circledR}$ Academic Research, v.11.0. The effect of altering the contact geometry and position on the displacement, electric field, stress, and strain distributions for the static case is reported.

Surface acoustic wave devices have drawn increasing interest for use as highly sensitive sensors. Specifically, SAW platforms are being explored for chemical and biological sensor applications. Because $\mathrm{AlN}$ has one of the highest acoustic velocities of all the piezoelectric materials, high frequency (and thus highly sensitive) sensors are feasible. In this work, AIN SAW Rayleigh wave platforms were designed, fabricated, and tested. The insertion loss of the SAW platforms for two InterDigitated Transducers (IDTs) separation distances is also presented. 


\section{ACKNOWLEDGMENTS}

I would like to acknowledge and thank the many people who helped to make this research possible. First, I would like to express my sincere appreciation to Dr. Dimitris Korakakis, my research advisor and committee chair, for his encouragement and guidance. He gave me the opportunity, freedom, and financial support to pursue the research topics that I found most interesting. I would like to thank Dr. Parviz Famouri, Dr. Lawrence Hornak, and Dr. Osama Mukdadi, my committee, for their technical help, support, and guidance. I would also like to thank Dr. David Lederman, Dr. Nianqiang Wu, Dr. Kolin Brown, Dr. Andrew Woodworth, and Mr. Eric Schires for their patience and help with the laboratory equipment. I am grateful to all my lab colleagues, both past and present, for their encouragement and help. In particular, I would like to acknowledge Adam Kabulski, Richard Farrell, John Harman, Joshua Nightingale, Sridhar Kuchibhatla, Kaylan Reddy Kasarla, Lee Rodak, Daniel Cortes, Cameron Keenan, and Smiti Bhattacharya. Without their valuable discussions and help in the lab, this work would have been impossible. Last, but not least, I would like to express my deep gratitude to my family and friends whose encouragement and support have been second to none. 


\section{TABLE OF CONTENTS}

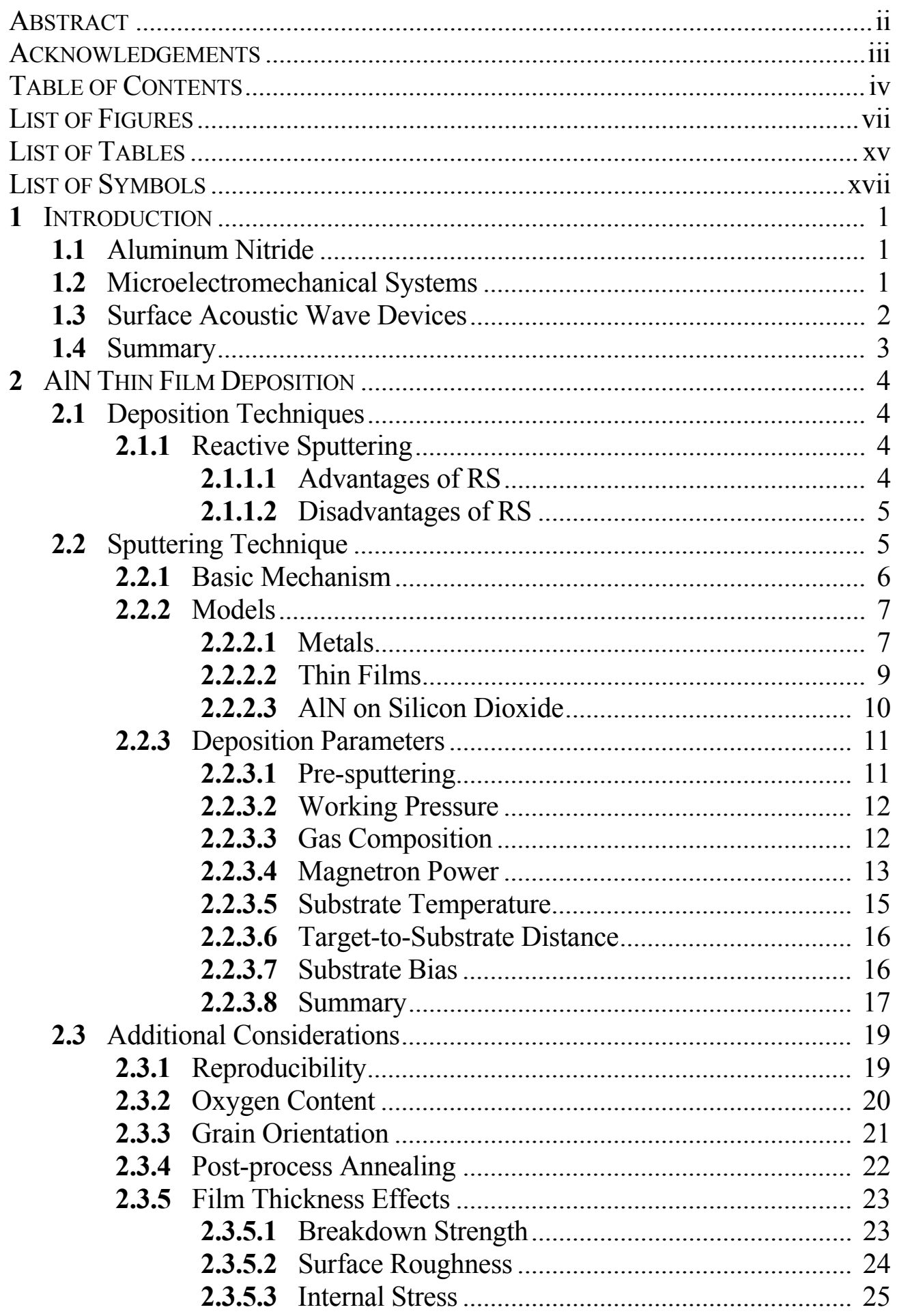


2.3.5.4 Crystallinity ………………………………….... 26

2.3.5.5 Piezoelectric Response ......................................... 27

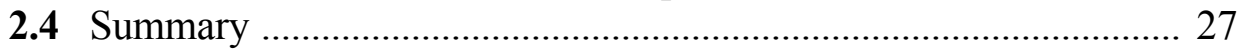

3 THIN FILM CHARACTERIZATION ……………………............................. 29

3.1 Characterization Techniques.......................................................... 29

3.1.1 AFM .................................................................... 29

3.1.1.1 Tapping Mode AFM.............................................. 30

3.1.1.2 van der Waals Force …………………………..... 31

3.1.1.3 Examples from Literature ...................................... 32



3.1.2.1 Effective Piezoelectric Coefficient ........................ 35

3.1.2.2 Effect of Contact Metal....................................... 37

3.1.2.3 Vibrational Modes ................................................ 38

3.1.3 Ellipsometry ……………………………………............. 39

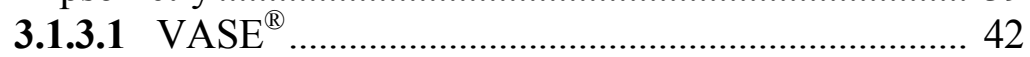

3.1.3.2 AlN Optical Model................................................ 43

3.1.3.3 Examples from Literature ....................................... 43

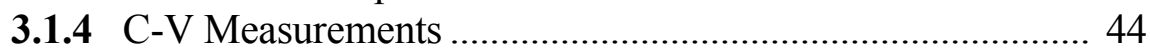

3.1.4.1 MIS Structures........................................................ 44

3.1.4.2 Silicon Electrical Properties ..................................... 46

3.1.4.3 Band Diagrams ..................................................... 47

3.1.4.4 Examples from Literature ………………………... 49

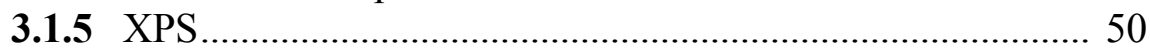

3.1.5.1 Operational Parameters ........................................... 51

3.1.5.2 Chemical Shift ……………………………….... 52

3.1.5.3 Elemental Composition........................................... 53

3.1.5.4 ARXPS ……………………………………..... 55

3.1.5.5 Destructive Depth Profiling …………………….... 56

3.1.5.6 Examples from Literature ………………………..... 56

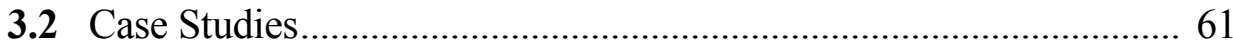

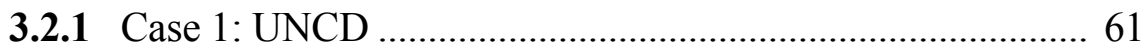



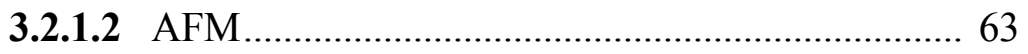



3.2.2 Case 2: AlN and Er-Doped AlN ........................................... 66

3.2.2.1 Deposition Parameters............................................ 67

3.2.2.2 Ellipsometry ......................................................... 68

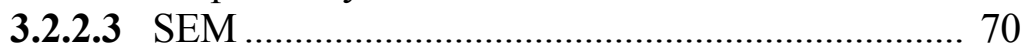

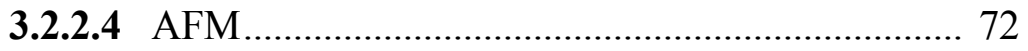

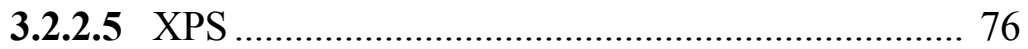

3.2.2.6 Summary ……………………………………...... 84

3.2.3 Case 3: AlN MIS Structures ................................................... 85

3.2.3.1 AlN Deposition........................................................ 85

3.2.3.2 Contact Deposition …………………………......... 86

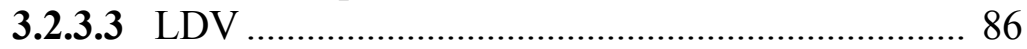


3.2.3.4 C-V Measurements................................................ 86

3.2.3.5 Summary …………………………………….... 91

4 Thin Film Piezoelectric DeVICES.......................................................... 93



4.1.1 Definition of Piezoelectricity ................................................. 93

4.1.2 Piezoelectric Materials....................................................... 95

4.1.3 Constituent Equations .......................................................... 95

4.1.4 Enhanced Piezoelectric Effect............................................. 98

4.1.4.1 Metal Contact Work Function .............................. 98

4.1.4.2 Rare Earth Doping ............................................... 99

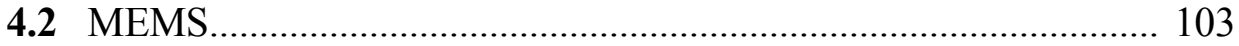

4.2.1 pMEMS ………………………………………........... 103

4.2.2 Piezoelectric Materials....................................................... 104

4.2.3 Piezoelectric Structures...................................................... 105

4.2.3.1 Electrode Configurations ....................................... 106

4.2.3.2 Diaphragms........................................................ 106

4.2.3.3 Piezoelectric Beams ........................................... 111

4.2.4 Summary ……………………………………….......... 122

5 Surface Acoustic Wave Devices ....................................................... 123

5.1 Background............................................................................. 123

5.1.1 Acoustic Waves................................................................... 123

5.1.2 Acoustic Wave Modes...................................................... 124

5.1.3 Surface Acoustic Waves ................................................... 125

5.1.4 SAW Materials................................................................. 125

5.1.5 SAW Devices................................................................... 126

5.1.5.1 SAW Delay Lines................................................. 127

5.1.5.2 Differential Sensors ............................................. 128

5.1.5.3 Acoustoelectric Convolvers .................................. 129

5.1.6 IDT Position .................................................................. 130

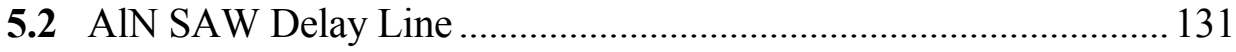

5.2.1 IDT Design ................................................................... 131

5.2.2 AlN Deposition ................................................................ 133

5.2.3 IDT Deposition ................................................................... 134

5.2.4 Dynamic Measurements ................................................... 136

5.2.5 Platinum Electrode Strips ................................................. 139

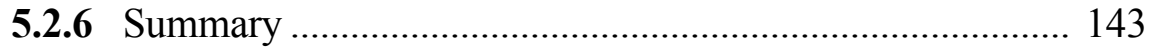

6 CONCLUSIONS AND FUtURE DIRECTIONS .................................................. 145

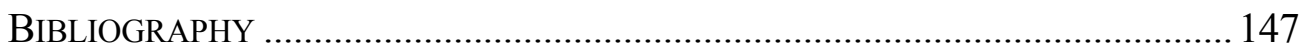

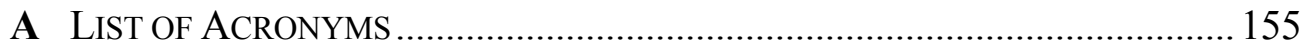

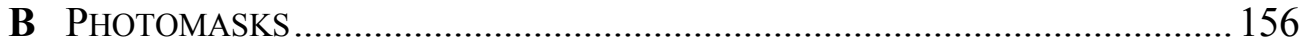



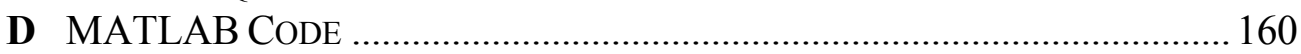

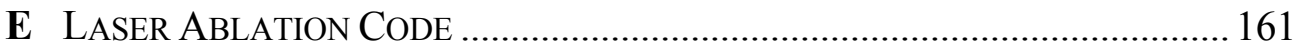

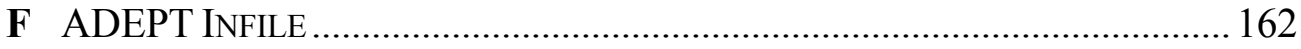

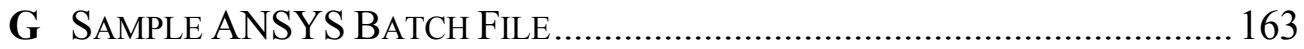




\section{LIST OF FIGURES}

Figure 2.1. Schematic of a typical sputtering system ..................................... 5

Figure 2.2. Sputtering energy transfer mechanisms. After Campbell

[Campbell, 2001] .................................................................... 7

Figure 2.3. Four zone structural model proposed by Thornton [Thornton,

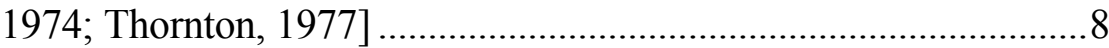

Figure 2.4. Structure of AlN films reactively sputtered $\mathrm{SiO}_{2} /(100) \mathrm{Si}$. After Meng [Meng, 1994]

Figure 2.5. Dependence of the film thickness of reactively sputtered AlN films on magnetron power

Figure 2.6. Dependence of the refractive index@588.947 nm of reactively sputtered AlN films on magnetron power.

Figure 2.7. Dependence of the surface roughness of reactively sputtered AlN

films on magnetron power

Figure 2.8. Dependence of the residual stress in reactively sputtered AlN films on substrate potential, flow rates, pressure, and magnetron power. After Dubois and Muralt [Dubois, 2001] 17

Figure 2.9. Oxidation of reactively sputtered AlN thin films. After Farrell et al. [Farrell, 2008] 23

Figure 2.10. Dependence of the breakdown strength of reactively sputtered AlN films on film thickness. After Liufu and Cao [Liufu, 1998]....24

Figure 2.11. Dependence of the roughness of reactively sputtered AlN films on film thickness. After Martin et al. [Martin, 2004] 25

Figure 2.12. Dependence of the residual stress of reactively sputtered AlN films on film thickness. After Martin et al. [Martin, 2004] 26

Figure 2.13. Dependence of the piezoelectric response of reactively sputtered AlN films on film thickness. After Kabulski et al. [Kabulski, 2009] 
Figure 3.1. Schematic of AFM beam deflection measurement system

[Nanoscience Instruments, 2008] 29

Figure 3.2. Plot of the Lennard-Jones 6-12 potential 31

Figure 3.3. Topography of $2 \mu \mathrm{m}$ thick reactively sputtered AlN film. (a) $1 \mu \mathrm{mx}$ $1 \mu m$ scan and (b) 5 um $x 5$ um scan. After Assouar et al. [Assouar, 2004]. 32

Figure 3.4. Topography of (a) $100 \mu \mathrm{m}$ thick polycrystalline diamond film and (b) $1 \mu \mathrm{m}$ thick reactively sputtered AlN film on silicon [Mortet, 2003] 33

Figure 3.5. Schematic of $L D V$ setup 34

Figure 3.6. Schematic of a Metal-Insulator-Semiconductor (MIS) structure 36

Figure 3.7. Displacement of a $1.1 \mu \mathrm{m}$ thick reactively sputtered AlN film with various metal top contacts. After Harman et al. [Harman, 2008]. 38

Figure 3.8. Vibrational modes of piezoelectric films with circular contacts. (a) regular modal shape and (b) distorted modal shape. After Yao et

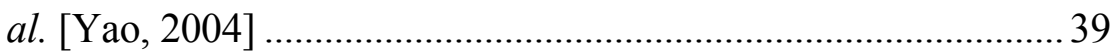

Figure 3.9. Reflection ellipsometry setup [Woollam, 2001] ............................ 40

Figure 3.10. Ellipsometric process flow for determining the optical properties of a material [Woollam, 2001]

Figure 3.11. Three layer model of a reactively sputtered AlN film on silicon. After Mahmood et al. [Mahmood, 2003]

Figure 3.12. Band diagram of Pt/ AlN/p-Si/ Pt MIS structure for an applied voltage of $0 \mathrm{~V}$ 47

Figure 3.13. Band diagram of Pt/ AlN/p-Si/ Pt MIS structure for an applied voltage of $5 \mathrm{~V}$

Figure 3.14. Band diagram of Pt/ AlN/p-Si/ Pt MIS structure for an applied voltage of $-5 \mathrm{~V}$. 48

Figure 3.15. Typical C-V curve of an Al/ AlN/p-Si/MIS capacitor. After Sharma et al. [Sharma, 2003] 49 
Figure 3.16. Direct photoelectron ejection mechanism. After Moulder et al.

[Moulder, 1995]

Figure 3.17. Relaxation mechanism resulting in the ejection of an Auger

photoelectron. After Moulder et al. [Moulder, 1995] 51

Figure 3.18. XPS survey scan spectra of reactively sputtered AlN. After

Engelmark et al. [Engelmark, 2000] 56

Figure 3.19. XPS survey scan spectra of reactively sputtered AlN. After

Sharma et al. [Sharma, 2003] 57

Figure 3.20. XPS detailed scan spectra of the Al2p peak of reactively sputtered AlN. After Sharma et al. [Sharma, 2003] .58

Figure 3.21. XPS depth profile of a columnar AlN film after annealing. After

Watanabe et al. [Watanabe, 2000] 58

Figure 3.22. XPS depth profile of a granular AlN film after annealing. After

Watanabe et al. [Watanabe, 2000] 59

Figure 3.23. Binding energies of the $3 d$ and $4 d$ electrons of rare earth ions. After Bernhardt et al. [Bernhardt, 2002]

Figure 3.24. $500 \mathrm{~nm} x 500 \mathrm{~nm}$ scan of an UNCD film. (a) 2-d topography image and (b) 3-d topography image

Figure 3.25. $2 \mu \mathrm{m} \times 2 \mu \mathrm{m}$ scan of an UNCD film. (a) 2-d topography image and (b) 3-d topography image 64

Figure 3.26. $5 \mu \mathrm{m} \times 5 \mu \mathrm{m}$ scan of an UNCD film. (a) 2-d topography image and (b) 3-d topography image

Figure 3.27. Schematic of an AlN thin film on a 3 " silicon wafer 66

Figure 3.28. Surface map of AlN films with a nominal thickness of $0.1 \mu \mathrm{m}$. (a) undoped and (b) Er-doped

Figure 3.29. Surface map of AlN films with a nominal thickness of $0.3 \mu \mathrm{m}$. (a) undoped and (b) Er-doped 69

Figure 3.30. Surface map of AlN films with a nominal thickness of $0.5 \mu \mathrm{m}$. (a) undoped and (b) Er-doped. 70 
Figure 3.31. Cross-sectional SEM images of AlN with a nominal thickness of $0.1 \mu \mathrm{m}$. (a) undoped $(t \approx 85 \mathrm{~nm})$ and (b) Er-doped $(t \approx 75 \mathrm{~nm})$.... 71

Figure 3.32. Cross-sectional SEM images of AlN with a nominal thickness of $0.3 \mu \mathrm{m}$. (a) undoped ( $t \approx 298 \mathrm{~nm})$ and (b) Er-doped $(t \approx 252 \mathrm{~nm}) 71$

Figure 3.33. Cross-sectional SEM images of AlN with a nominal thickness of $0.5 \mu \mathrm{m}$. (a) undoped $(t \approx 375 \mathrm{~nm})$ and (b) Er-doped $(t \approx 274 \mathrm{~nm}) 72$

Figure 3.34. Topography of AlN with a nominal thickness of $0.1 \mu \mathrm{m}$. (a) undoped and (b) Er-doped. 73

Figure 3.35. Topography of AlN with a nominal thickness of $0.3 \mu \mathrm{m}$. (a) undoped and (b) Er-doped..... 73

Figure 3.36. Topography of AlN with a nominal thickness of $0.5 \mu \mathrm{m}$. (a) undoped and (b) Er-doped 74

Figure 3.37. Measured surface roughness as a function of film thickness for undoped and Er-doped AlN films .75

Figure 3.38. Measured grain height as a function of film thickness for undoped and Er-doped AlN films 75

Figure 3.39. XPS survey scan of an undoped AlN film with a nominal thickness of $0.1 \mu \mathrm{m}$ 76

Figure 3.40. XPS survey scan of an undoped AlN film with a nominal thickness of $0.3 \mu \mathrm{m}$ 77

Figure 3.41. XPS survey scan of an undoped AlN film with a nominal thickness of $0.5 \mu \mathrm{m}$.

Figure 3.42. XPS survey scan of an Er-doped AlN film with a nominal thickness of $0.1 \mu \mathrm{m}$. 78

Figure 3.43. XPS survey scan of an Er-doped AlN film with a nominal thickness of $0.3 \mu \mathrm{m}$. 78

Figure 3.44. XPS survey scan of an Er-doped AlN film with a nominal thickness of $0.5 \mu \mathrm{m}$

Figure 3.45. XPS depth profile of an undoped AlN film with a nominal thickness of $0.1 \mu \mathrm{m}$ 81 
Figure 3.46. XPS depth profile of an undoped AlN film with a nominal thickness of $0.3 \mu \mathrm{m}$.

Figure 3.47. XPS depth profile of an undoped AlN film with a nominal thickness of $0.5 \mu \mathrm{m}$. (Note: the number of data points shown in the plot was reduced by a factor of 3)

Figure 3.48. XPS depth profile of an Er-doped AlN film with a nominal thickness of $0.1 \mu \mathrm{m}$. 82

Figure 3.49. XPS depth profile of an Er-doped AlN film with a nominal thickness of $0.3 \mu \mathrm{m}$

Figure 3.50. XPS depth profile of an Er-doped AlN film with a nominal thickness of $0.5 \mu \mathrm{m}$. (Note: the number of data points shown in the plot was reduced by a factor of 3)

Figure 3.51. Typical C-V curve of an Al/ AlN/p-Si/ Al structure with a $1 \mu \mathrm{m}$ AlN insulating layer

Figure 3.52. Typical C-V curve of a Pt/ AlN/ p-Si/ Al structure with a $1 \mu \mathrm{m}$ AlN insulating layer

Figure 3.53. Typical C-V curve of an Al/ AlN/p-Si/ Pt structure with a $1 \mu \mathrm{m}$ AlN insulating layer

Figure 3.54. Typical C-V curve of a Pt/ AlN/ p-Si/ Pt structure with a $1 \mu \mathrm{m}$ AlN insulating layer

Figure 3.55. Measured charge and measured effective longitudinal piezoelectric coefficient for four types of MIS structures

Figure 3.56. Measured effective longitudinal piezoelectric coefficient as a function of applied dc offset for four types of MIS structures

Figure 3.57. Band diagrams of heterogeneous LEDs fabricated by growing AlN on p-Si wafers. (a) zero applied bias, (b) negative applied bias, and (c) positive applied bias. The bias polarity was defined in the terms of the Au/AlN interface [Zhao, 2009] 92

Figure 4.1. Stress-strain curves for a brittle and ductile material [Campbell, 2001]. 
Figure 4.2. Effective piezoelectric strain coefficient of various AlN thin films. After Harman et al. [Harman, 2008] . 99

Figure 4.3. Photoluminescence spectra of Er-doped reactively sputtered AlN films. After Brien et al. [Brien, 2007] 101

Figure 4.4. Various types of defects occurring in a crystal. (A) vacancies, (B) self-interstitials, (C) substitutional impurities, (D) edge dislocations, and (E) dislocation loops. After Campbell [Campbell, 2001]. 102

Figure 4.5. Common piezoelectric structures 105

Figure 4.6. Common electrode configurations. (a) planar capacitor and (b) interdigitated transducer. After Trolier-McKinstry and Muralt [Trolier-McKinstry, 2004]

Figure 4.7. Cantilever and diaphragm based microvalve system. After Koch et al. [Koch, 1996] 107

Figure 4.8. SEM images of diaphragms fabricated with a circular mask. (a) backside, $a_{\text {diaphragm }} \approx 43 \mu \mathrm{m}$, and (b) backside, $a_{\text {diaphragm }} \approx 230 \mu \mathrm{m}$

Figure 4.9. SEM images of diaphragms fabricated with the square diaphragm mask (see Appendix B). (a) cross-sectional image and (b) backside

Figure 4.10. SEM images of pure silicon diaphragms fabricated with the square diaphragm mask (see Appendix B). (a) backside and (b) corner

Figure 4.11. Various cantilever configurations. After Chapuis et al. [Chapuis, 2006].

Figure 4.12. Serial bimorph. After Huang et al. [Huang, 2004] 113

Figure 4.13. Parallel bimorphs. (a) parallel and (b) bipolar parallel. After Huang et al. [Huang, 2004]. 
Figure 4.14. Optical microscope images of a laser ablated, diaphragm based cantilever. (a) topside image before solvent bath and (b) backside image after solvent bath 115

Figure 4.15. (a) Bulk micromachined diaphragm structure and (b) cantilever structure simulated using finite element analysis

Figure 4.16. Bimorph deflection along the "Mid" path ............................... 120

Figure 4.17. Bimorph deflection along the "Edge" path .............................. 120

Figure 4.18. Bimorph deflection along the "Tip" path ............................... 121

Figure 4.19. Derivative with respect to position of the deflection along the

"Mid" path (see Figure 4.15(b))

Figure 5.1. Longitudinal wave. After Krautkrämer and Krautkrämer [Krautkrämer, 1990] 124

Figure 5.2. Transverse wave. After Krautkrämer and Krautkrämer [Krautkrämer, 1990] 124

Figure 5.3. SAW two port delay line. After Pearce et al. [Pearce, 1981]..... 127

Figure 5.4. SAW velocity dispersion curves. (a) $2.8 \mu \mathrm{m}$ high quality AlN, (b) $1.0 \mu \mathrm{m}$ high quality AlN, (c) and (d) 1.0 um lower quality AlN. After Hickernell [Hickernell, 1995].... 128

Figure 5.5. $S A W$ differential sensor. The gold colored region in between the IDTs. After Branch and Brozik [Branch, 2004]

Figure 5.6. SAW acoustoelectric convolver. After Pearce et al. [Pearce, 1981]

Figure 5.7. Two common IDT placements. After Nakahata et al. [Nakahata, $1995]$ 130

Figure 5.8. IDT pattern used in this work 132

Figure 5.9. Rayleigh-wave mode $S A W$ delay line fabricated in this work .... 134

Figure 5.10. SEM images of an AIN SAW delay line with shorted IDTs. (a) 50 times magnification of entire device and 180 times magnification of IDTS 135 
Figure 5.11. SEM images of an AlN SAW delay line. (a) 40 times magnification of entire device and 200 times magnification of IDTs

Figure 5.12. SEM images of IDTs on AlN. (a) $1.00 \mathrm{k}$ times magnification of top IDT and (b) $1.00 \mathrm{k}$ times magnification of bottom IDT

Figure 5.13. I/O response of a $S A W$ delay line consisting of a $300 \mathrm{~nm}$ AlN thin

$$
\text { film, freq }=50 \mathrm{MHz}
$$

Figure 5.14. I/O response of a $S A W$ delay line consisting of a $300 \mathrm{~nm}$ AlN thin film, 20 ns pulse 138

Figure 5.15. I/O response of a $S A W$ delay line consisting of a $300 \mathrm{~nm}$ AlN thin film, 20 ns pulse (zoomed) 138

Figure 5.16. I/O response of a $S A W$ delay line consisting of a $300 \mathrm{~nm}$ AlN thin film, freq $=100 \mathrm{kHz}$. 139

Figure 5.17. SEM images of an AlN SAW delay line with a platinum electrode strip. (a) 45 times magnification of entire device and (b) 200 times magnification of IDTs 140

Figure 5.18. I/O response of a $S A W$ delay line consisting of a $300 \mathrm{~nm}$ AlN thin film and a $70 \mathrm{~nm}$ platinum electrode strip, $250 \mu \mathrm{m}$ separation, freq $=100 \mathrm{kHz}$

Figure 5.19. I/O response of a $S A W$ delay line consisting of a $300 \mathrm{~nm}$ AlN thin film and a $70 \mathrm{~nm}$ platinum electrode strip, $180 \mu \mathrm{m}$ separation, freq $=100 \mathrm{kHz}$

Figure 5.20. I/O response of a $S A W$ delay line consisting of a $300 \mathrm{~nm}$ AlN thin film and a $70 \mathrm{~nm}$ platinum electrode strip, $180 \mu \mathrm{m}$ separation, freq $=100 \mathrm{kHz}$. The sinusoidal signals are the normalized output when the platinum strip is biased at $0 \mathrm{~V}$ and $25 \mathrm{~V}$. The ratio of these two signals is also plotted. 142

Figure 5.21. Comparison of $S A W$ devices with and without a platinum electrode strip 143 


\section{LIST OF TABLES}

Table 2.1. Summary of the deposition parameters reported in the literature for reactively sputtering AlN thin films..... 18

Table 2.2. Summary of the optimal deposition parameters found for reactively sputtering AlN films using a CVC 610 DC magnetron sputter deposition system 18

Table 3.1. Atomic sensitivity factors or spectral lines of relevance to this work. After Moulder et al. [Moulder, 1995].

Table 3.2. Binding energies of rare earth ions. After Bernhardt et al.

[Bernhardt, 2002] 60

Table 3.3. Optical model for UNCD over the spectral range of $400 \mathrm{~nm}$ to 1000 $n m$ 62

Table 3.4. Optical model for UNCD over the spectral range of $700 \mathrm{~nm}$ to 1000 $n m$

Table 3.5. Pre-sputtering and sputtering parameters 67

Table 3.6. Information derived using VASE ${ }^{\circledR}$..... 68

Table 4.1. Coefficients of various piezoelectrics. After Trolier-McKinstry and Muralt [Trolier-Mckinstry, 2004] 95

Table 4.2. Constituent equations for a piezoelectric material. After Jaffe [Jaffe, 1965; Jaffe, 1971] 96

Table 4.3. Symbols and units for electroelastic constants. After Jaffe [Jaffe, 1965]

Table 4.4. Electroelastic matrix for the 6mm crystal class 98

Table 4.5. Material properties of RE-doped PZT films. After Shannigrahi et al.[Shannigrahi, 2004] 100

Table 4.6. $A N S Y S^{\circledR}$ Results Viewer plots of the electric potential and total strain intensity of the "Full Contact" and the "Half Contact" .. 117 
Table 4.7. ANSYS ${ }^{\circledR}$ Results Viewer plots of the electric potential and total strain intensity of the "Diamond Shaped Contact" and the "T Shaped Contact"

Table 4.8. $A N S Y S^{\circledR}$ Results Viewer plots of the electric potential and total strain intensity of the "Cantilever Shaped Contact" and "Vertical Contacts" 118

Table 4.9. $A N S Y S^{\circledR}$ Results Viewer plots of the electric potential and total strain intensity of "Vertical Contacts (4/3)" " and "Vertical Contacts $(3 / 2)^{n,}$ 119

Table 4.10. Maximum values of the deflection, stress, strain, and area for the contacts defined in Tables 4.6-4.9 122

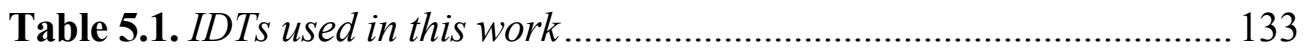




\section{LIST OF SYMBOLS}

$\begin{array}{lll}\varepsilon & : & \text { Dielectric constant (or permittivity) } \\ \varepsilon & : & \text { Strain } \\ \rho & : & \text { Density } \\ \sigma & : & \text { Stress } \\ \beta & : & \text { Wavenumber } \\ \lambda & : & \text { Wavelength } \\ \Gamma & : & \text { Reflection coefficient } \\ & & \\ c & : & \text { Elastic stiffness constant } \\ d & : & \text { Piezoelectric strain constant } \\ e & : & \text { Piezoelectric stress constant } \\ f & : & \text { Frequency } \\ k & : & \text { Electromechanical coupling coefficient } \\ k & : & \text { Wavenumber } \\ p & : & \text { Pyroelectric coefficient } \\ S & : & \text { Elastic compliance constant } \\ t & : & \text { Thickness } \\ v_{p} & : & \text { Phase velocity } \\ v_{s} & : & \text { Sound velocity } \\ D & & \\ E & : & \text { Electric flux density } \\ Q & : & \text { Electric field } \\ S & : & \text { Quality factor } \\ S & : & \text { Scattering parameter } \\ T & : & \text { Strain } \\ Y & : & \text { Stress } \\ Y & : & \text { Admittance } \\ & : & \text { Young's modulus } \\ & & \end{array}$




\section{H A P T E R 1: I N T R O D U C T I O N}

This work addresses the deposition and characterization of AlN thin films and their application in the design and fabrication of pMEMS/SAW devices. Additionally, methods of enhancing the piezoelectric response of AlN based devices are considered.

\subsection{AluminUm NitRide}

Aluminum Nitride (AIN) is a III-V semiconductor material [Carlotti, 1995] that grows in the wurzite crystal structure [Martin, 2004]. AlN is a direct wide bandgap semiconductor with a bandgap, $E_{g}$ of $6.2 \mathrm{eV}$ [Carlotti, 1995; Elmazria, 2003]. It is a promising material for piezoelectric MicroElectroMechanical Systems (pMEMS) and Surface Acoustic Wave (SAW) devices due to its high acoustic velocity and favorable electrical, mechanical, and chemical properties [Benetti, 2007]. The advantages of using AlN include a perfect compatibility with silicon semiconductor technology [TrolierMckinstry, 2004], chemical stability [Sharma, 2003; Iriarte, 2003; Cheng, 2003; Caliendo, 1993], a wide bandgap [Trolier-Mckinstry, 2004; Iriarte, 2003; Caliendo, 1993], high thermal conductivity [Sharma, 2003; Iriarte, 2003], high thermal stability [Sharma, 2003], high dielectric strength [Sharma, 2003], and excellent acoustic properties [Clement, 2003]. In addition, since AlN is completely compatible with current silicon and Complementary Metal-Oxide-Semiconductor (CMOS) technologies, it is possible to develop Integrated Circuits (ICs) and MEMS on a single chip [Carrillo, 2003].

Some of the common applications for AIN thin films include SAW devices operating in the megahertz $(\mathrm{MHz})$ and the gigahertz $(\mathrm{GHz})$ range [Tsubouchi, 1985], Bulk Acoustic Wave (BAW) resonators and filters [Wang, 2005], MEMS devices [Iborra, 2004], electrical insulation [Elmazria, 2003; Mahmood, 2003], and optoelectronic devices such as Light Emitting Diodes (LEDs) and Laser Diodes (LDs) [Shimada, 1998; Elmazria, 2003].

\subsection{MicROELECTROMECHANICAL SYSTEMS}

MicroElectroMechanical Systems (MEMS) are microscopic devices that interact with their environment. They typically translate an electrical stimulus into a mechanical 
response, or vice versa [Walraven, 2003]. MEMS devices offer several advantages over their bulk counterparts which include reduced power consumption, reduced weight, lower cost and in some cases improved performance [Walraven, 2003] In addition, MEMS devices can be fabricated using traditional semiconductor manufacturing techniques [Carrillo, 2003]. This means that current micromachining techniques such as photolithography, pattern transfer by etching, and thin film deposition techniques used to fabricate modern integrated circuits can be employed to fabricate silicon ( $\mathrm{Si}$ ) based MEMS devices [Baborowski, 2004]. Finally, since the current semiconductor technology is based on silicon, hybrid devices that incorporate integrated circuits and MEMS functionality can be fabricated on the same chip.

\subsection{Surface Acoustic Wave Devices}

Acoustic waves have been used for many years to determine the mechanical properties of materials. For example, internal defects have been detected in forgings and castings for centuries by observing the ringing tone generated when struck by a hammer [Krautkrämer, 1990]. Acoustic waves can be described as the periodic mechanical disturbances of particles composing the propagation medium. Surface acoustic waves (SAWs) travel near the surface of the propagation medium. SAWs typically experience a strong reduction in amplitude as a function of distance from the interface [Kallard, 1971]. The penetration depth of the SAWs decreases as the frequency is increased. Thus, the surface sensitivity is increased as a function of the square of the frequency [Gronewold, 2007]. The wave velocity and amplitude are parameters commonly measured to determine interactions such as mass loading, viscosity, and changes in conductivity [Gronewold, 2007; Länge, 2008]. Thus, SAW devices have much potential as chemical and biological sensors.

\subsection{SUMMARY}

In this work, the deposition and characterization of AlN thin films is reported. Properties of the films such as the crystallinity, effective piezoelectric coefficient, and surface roughness were optimized for use in pMEMS and SAW devices. AIN based 
diaphragm structures were fabricated and AIN cantilevers were simulated. Finally, AlN Rayleigh wave mode SAW devices were developed and tested at frequencies up to 50 MHz. 


\section{H A P T E R 2: A $1 \mathrm{~N}$ T H I N F I L M D E P O S I T I O N}

\subsection{Deposition TeChNiQueS}

Aluminum nitride thin films have been prepared using a variety of techniques. The most common include Chemical Vapor Deposition (CVD) [Iriarte, 2003; Muhl, 1997; Fujiki, 2000], thermal evaporation [Iriarte, 2003; Fujiki, 2000], Molecular Beam Epitaxy (MBE) [Iriarte, 2003; Muhl, 1997], Pulsed Laser Deposition (PLD) [Dubois, 2001], ion beam nitridation [Fujiki, 2000], and Reactive Sputtering (RS) [Iriarte, 2003; Muhl, 1997; Fujiki, 2000].

\subsubsection{REACTIVE SPUTTERING}

Reactive sputtering is an inexpensive and low-temperature technique for producing good quality polycrystalline films [Fujiki, 2000]. Highly-oriented, polycrystalline AlN films with piezoelectric responses similar to those of epitaxial films have been deposited using RS [Clement, 2003]. The advantages and disadvantages of this technique for the deposition of AlN films are discussed next.

\subsubsection{ADVANTAGES OF RS}

Reactive sputtering offers many advantages for the deposition of AlN films. Reactive sputtered films are generally smoother than films grown by CVD. This is mainly because of the high-temperatures $\left(>1000 \mathrm{C}^{\circ}\right)$ required for the CVD growth of AlN on sapphire [Iriarte, 2003]. This gives rise to another advantage of using RS for the deposition of AlN films. The use of sapphire substrates in growth techniques including CVD result in an incompatibility with silicon micromachining processes. Since AlN can be deposited directly on silicon substrates with RS [Dubois, 2001], any subsequent silicon processing steps can still be performed. Additionally, AlN can be reactively sputtered on materials or structures that are unable to withstand the high-temperatures used in CVD, PLD, and MBE techniques [Dubois, 2001]. 


\subsubsection{DisADVANTAGES OF RS}

Aluminum nitride films deposited using RS are typically of lesser quality than those grown using CVD or MBE. Additionally, the mechanics for depositing AlN using RS are not fully understood which makes optimizing the deposition parameters difficult. Proper selection of the deposition parameters is essential for depositing AIN films with a polycrystalline orientation along the $c$-axis.

\subsection{SPUTtering TeChNiQue}

Sputtering is a plasma based process in which material from an ion bombarded target is ejected onto a substrate. The process is reactive if the ejected material reacts with the gas inside the sputtering chamber. A typical sputtering station consists of a vacuumsealed chamber, vacuum pump(s), gas inlets and valves, gauges and controls, sputter gun(s), and a power supply as shown in Figure 2.1.

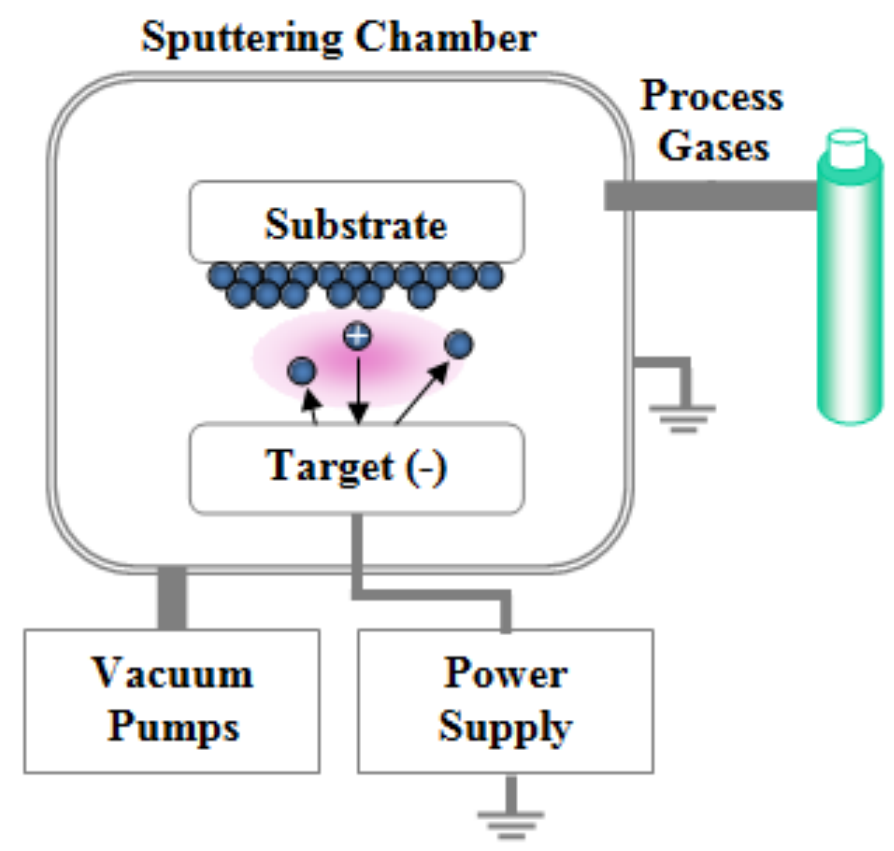

Figure 2.1. Schematic of a typical sputtering system. 


\subsubsection{BASIC MECHANISM}

Since sputtering is a plasma based technique, a basic knowledge of plasma is advantageous. Plasma is the fourth state of matter. It is a partially ionized gas that consists of free moving electrons and ions. Plasma is ignited in a sputtering chamber by applying a voltage greater than the breakdown voltage across two electrodes surrounding a lowpressure gas. Common process gases used in sputtering include nitrogen $\left(\mathrm{N}_{2}\right)$, oxygen $\left(\mathrm{O}_{2}\right)$, krypton $(\mathrm{Kr})$, and argon (Ar). The breakdown voltage of the gas is given by Paschen's law (2.1) where $P$ is the chamber pressure, $L$ is distance between the two electrodes, and $b$ is a constant [Campbell, 2001].

$$
V_{b d} \propto \frac{P \times L}{\log P \times L+b}
$$

Due to the potential difference between the chamber sidewalls and the target, the ions in the plasma are accelerated toward the target situated over the sputter gun cathode. The ions strike the target surface releasing secondary electrons which are then repelled away from the cathode. Energy from the secondary electrons can be transferred to neutral plasma species via collision events. The transferred energy can either ionize or excite the species to energetic states. In the former case, the ionized species are accelerated toward the target/sputter gun cathode. In the latter case, the energetic atoms decay to their ground states through light emitting transitions [Campbell, 2001].

Several energy transfer mechanisms are possible when an energetic ion strikes the target as shown in Figure 2.2. Low energy ions $(E<10 \mathrm{eV})$ may bounce off or adsorb to the surface of the target causing target heating. High energy ions $(E>10 \mathrm{keV})$ may be implanted deep within the target causing physical rearrangement of the target material. Ions with moderate energies may be involved in a combination of low and high energy transfer mechanisms. However, instead of penetrating deep into the target (as in the high energy case), they are typically stopped through nuclear interactions within a distance of a few atomic layers into the target material. In this case, the target material is ejected with energies between 10 and $50 \mathrm{eV}$. Typically, $95 \%$ of the target material is ejected in the form 
of atoms while the remaining $5 \%$ is ejected as small particles of target material [Campbell, 2001].

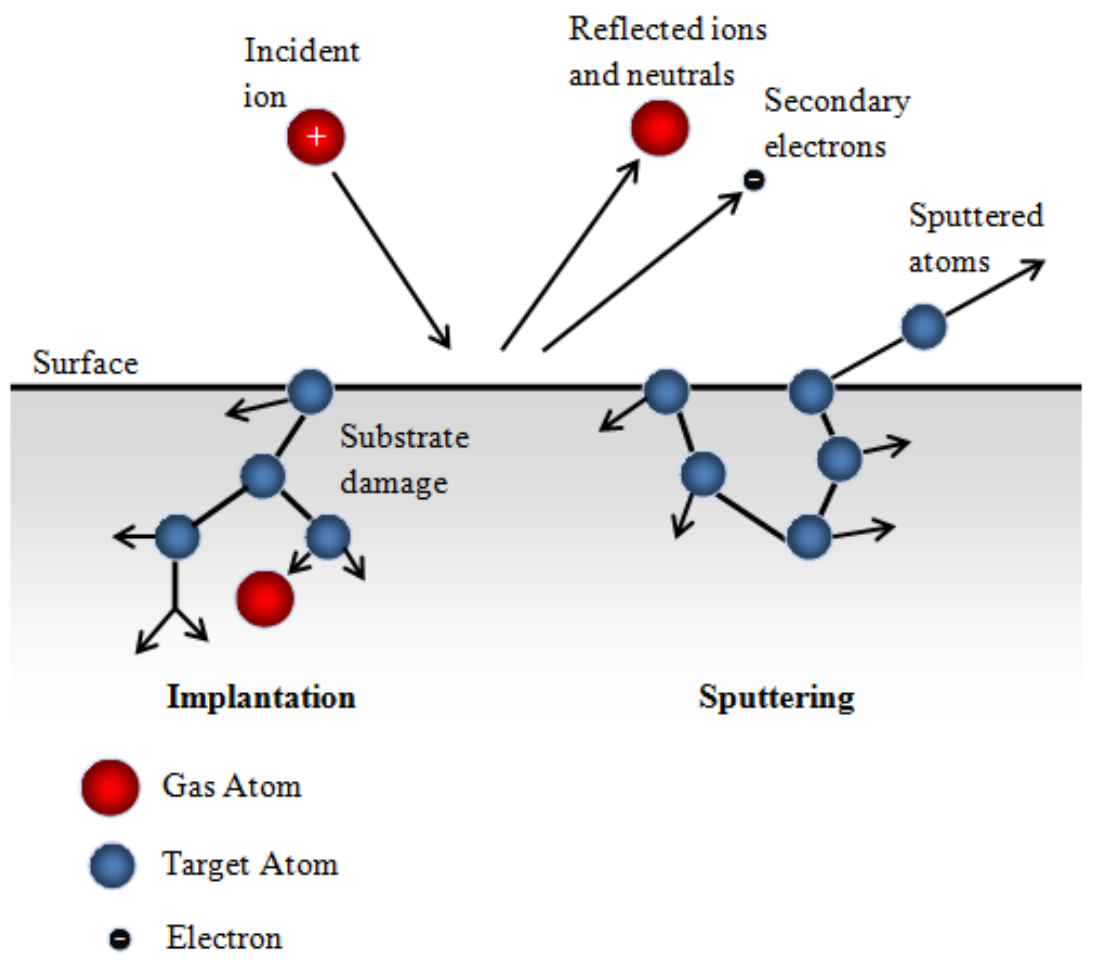

Figure 2.2. Sputtering energy transfer mechanisms. After Campbell [Campbell, 2001].

\subsubsection{MODELS}

Various models of film deposition using reactive sputtering have been proposed. Three models will be presented in this section. The first addresses the deposition of relatively thick metals ( 25 to $250 \mu \mathrm{m}$ ), the second addresses the deposition of thin films, and the third addresses the reactive deposition of AIN on silicon dioxide. A basic familiarity with these models is helpful when determining the optimal sputtering deposition parameters.

\subsubsection{METALS}

Thornton proposed a model for the depositions of thick metal films based off of the zone model proposed by Movchan and Demchishin [Thornton, 1977]. Metal films of 
titanium, chromium, iron, copper, molybdenum, and aluminum alloy were deposited on metal and glass substrates. The thickness of the metal films ranged from 25 to $250 \mu \mathrm{m}$. Four zones were defined as shown in Figure 2.3 where $T$ is the substrate temperature and $T_{m}$ is the melting temperature of the target material.

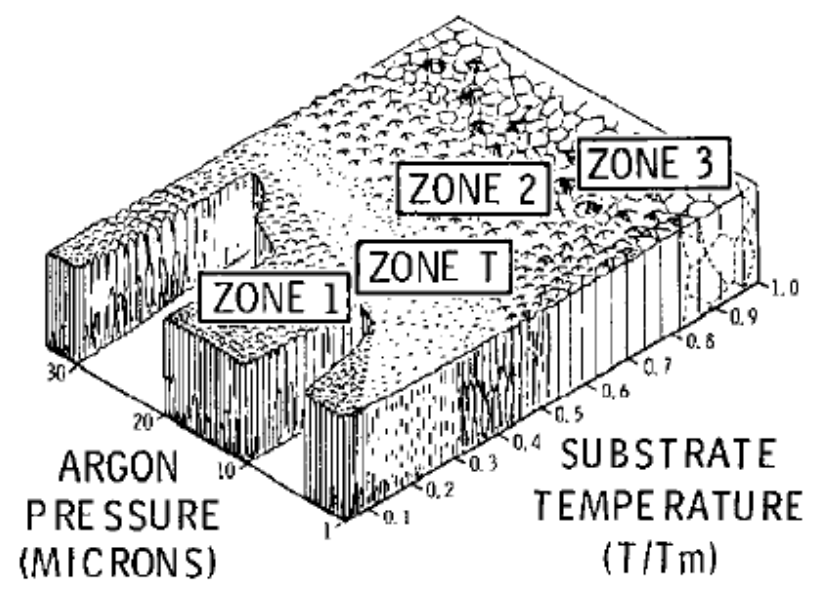

Figure 2.3. Four zone structural model proposed by Thornton [Thornton, 1974; Thornton, 1977].

The Zone 1 structure was characterized by an exaggerated substrate roughness and a dense, fibrous appearance. Films having this structure were obtained when high argon pressures and low temperatures ( $T / T_{m}<0.3$ ) were used. The Zone T (or "transition zone") structure was characterized by a smooth surface and a dense, poorly defined, fibrous appearance. This structure was reported to be the limiting case for the Zone 1 structure when a perfectly smooth substrate was used. Zone 1 and Zone T structures comprise the regime in which surface diffusion of loosely bonded "adatoms" (defined in Section 2.2.2.2) are limited by geometric considerations such as "shadowing." Shadowing is a geometric interaction in which the roughness on the surface of the growing films blocks incoming material arriving at certain angles with respect to the substrate [Thornton, 1977].

The Zone 2 structure was characterized by a highly faceted surface and dense, columnar grains. The grain size was found to increase and the surface roughness was found to decrease with increasing $T / T_{m}$. The Zone 2 structure comprises the regime in 
which the film deposition process is dominated by surface diffusion of the adatoms [Thornton, 1977].

The Zone 3 structure was characterized by broad columnar and/or "equiaxed" grains. The Zone 3 structure is typically produced at $T / T_{m}$ between 0.5 and 1 and comprises the regime in which the film deposition process is dominated by bulk diffusion. The relationship between the formation of columnar and equiaxed grains is not fully understood [Thornton, 1977].

\subsubsection{THIN FILMS}

The process of depositing thin films of compound materials such as AlN is more complicated than the process of sputtering pure metals. The growth of thin films generally occurs in a series of four stages [Pashley, 1964]. The four stages are 1) nucleation and island structure, 2) coalescence of islands, 3) channel formation and 4) continuous film formation [Iriarte, 2003].

In the first stage, atoms absorbed on the surface of the deposited film ("adatoms") collect and form clusters called nuclei. The result is a number of separate islands. The nuclei continue grow until they reach a critical nuclei size. If the critical nucleus size is large and the energy of formation is high, the grain of the film will be coarse; likewise, if the critical nucleus size is small and the energy of formation is high, the grain of the film will be fine. In the second stage, the islands of nuclei combine to form a continuous film. This is attributed to the increased nuclei density on the surface of the substrate. Nuclei with well-defined crystallographic shapes tend to become rounded during this stage. In the third stage, the islands form networks of narrow channels of elongated islands. This produces an alignment of islands along the crystal structures in the growing film. Finally, in the fourth state, the orientation of the islands changes which gives rise to various degrees of recrystallization in the film. The final grain size of the film is determined primarily by the recrystallization that occurs in this stage [Iriarte, 2003]. 


\subsubsection{AlN ON SILICON DiOXIDE}

The deposition of AlN thin films on silicon dioxide $\left(\mathrm{SiO}_{2}\right)$ films was investigated by Meng et al. AlN films reactively sputtered on $\mathrm{SiO}_{2} /(100) \mathrm{Si}$ were characterized using X-Ray Diffraction (XRD), Convergent Beam Electron Diffraction (CBED), Transmission Electron Microscopy (TEM), and Scanning Electron Microscopy (SEM) [Meng, 1994]. The films were found to consist of four separate layers distinguished by their crystalline phase and grain orientation. The layers, as ordered from the closest to the farthest from the substrate, are reaction, transition, alignment and surface as shown in Figure 2.4.

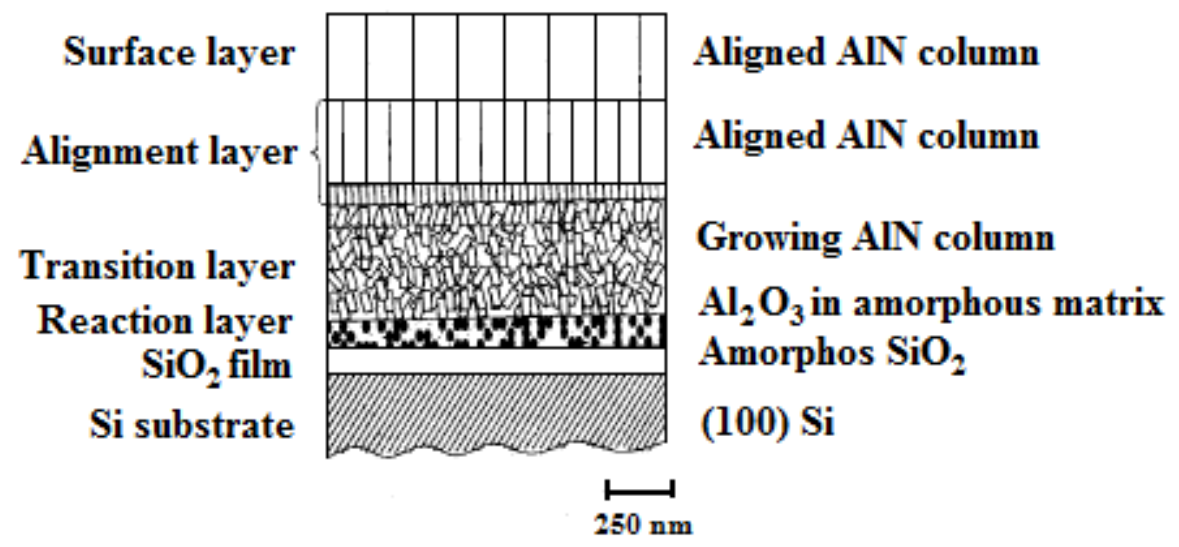

Figure 2.4. Structure of AlN films reactively sputtered on $\mathrm{SiO}_{2} /(100) \mathrm{Si}$. After Meng [Meng, 1994].

Meng's results indicated the formation of a thin amorphous $\mathrm{Al}_{2} \mathrm{O}_{3}$ layer at the $\mathrm{AlN} / \mathrm{SiO}_{2}$ interface. This was attributed to the residual oxygen present in the chamber during deposition. It is also possible that the oxygen from the $\mathrm{SiO}_{2}$ layer reacted with sputtered aluminum atoms. Their results show that the epitaxial growth of AlN thin films on $\mathrm{SiO}_{2}$ is inhibited. However, polycrystalline growth was observed in the AlN film above the amorphous $\mathrm{Al}_{2} \mathrm{O}_{3}$ layer. So, if the piezoelectric response is a function of grain orientation (discussed in Section 2.3.3) the piezoelectric response of AlN films deposited on silicon dioxide most likely differ from the piezoelectric response of AIN films deposited directly on silicon. 


\subsubsection{DEPOSITION PARAMETERS}

A brief survey of the deposition parameters found in the literature for reactively sputtering AlN thin films is presented in this section. A large variance in the deposition parameters used by different research groups was found. This variance is more than likely due to differences in the sputtering system setups used and the complexity of plasma based processes. The deposition parameters that influence the quality and structure of reactively sputtered AlN thin films are 1) the inclusion of a pre-sputtering step, 2) working pressure, 3) gas composition, 4) magnetron power, 5) substrate temperature, 6) target-to-substrate distance, and 7) substrate bias voltage.

\subsubsection{PRE-SPUTTERING}

The importance of incorporating an adequate pre-sputtering step cannot be overstated. Pre-sputtering serves three purposes. First, the targets used for reactively sputtering AlN films are typically made of pure aluminum. Aluminum is known to oxidize in ambient environments. Thus, a thin layer of aluminum oxide $\left(\mathrm{Al}_{2} \mathrm{O}_{3}\right)$ will almost always be present on the target surface. Second, impurities can be deposited on the target surface during sputtering. Pre-sputtering with a noble gas such as argon removes the oxide layer and other impurities reducing their incorporation in the sputtered film. Third, presputtering with a mixture of deposition gases such as argon and nitrogen can be used to form a surface layer of AlN on the target. This process is sometimes referred to "poisoning" the target [Boeshore, 2005].

Various pre-sputtering processes have been reported in the literature. Mahmood et al. included a 5 to 10 minute pre-sputter step using nominal deposition conditions [Mahmood, 2003] while Jones et al. included a 60 minute pre-sputter step using only argon gas [Jones, 1999]. We have found that a two-step pre-sputter process provides the best results. First the target is pre-sputtered for 10 minutes using only argon gas to remove the oxide layer and impurities. The target is then sputtered for an additional 5 minutes in a mixture of argon and nitrogen gases to poison the target. 


\subsubsection{WORKING PRESSURE}

The working pressure has been reported to affect the deposition by influencing the mean free path of the discharge gas [Clement, 2003]. The mean free path and the working pressure have been shown to be inversely proportional to each other by considering the kinetic theory of gases [Iriarte, 2003]. At lower pressures, the mean free path of the gas atoms is extended since the scattering probability of the reactive species in the gas is decreased [Cheng, 2003]. A higher density of energetic particles will impinge on the substrate surface if the mean free path is increased or the substrate to target distance is decreased. This increases the amount of energy transferred from the gas particles to the adatoms on the surface of the substrate. Higher adatom energies are reported to promote AlN film growth along the (002) preferred orientation [Cheng, 2003; Clement, 2003]. At higher pressures (which leads to a reduction of adatom energy) the film structures have been found to be dominated by the (100) orientation [Cheng, 2003]. These findings suggest that low working pressures are necessary in order to deliver adequate energy to the adatoms on the growing film surface.

\subsubsection{GAS COMPOSITION}

The gas composition of nitrogen $\left(\mathrm{N}_{2}\right)$ to argon (Ar) has been reported to affect the stoichiometry and structure of the film [Iriarte, 2003]. The plasma generated while reactively sputtering aluminum nitride is composed of electrons, argon and nitrogen atoms, divalent nitrogen molecules, and argon and nitrogen ions. One of the results of varying the gas composition is a change in the cation composition. Since the argon ions $\left(\mathrm{Ar}^{+}\right)$carry a higher momentum than either of the nitrogen ions $\left(\mathrm{N}^{+}, \mathrm{N}^{2+}\right)$, a reduction in the argon composition decreases the energy transferred to the substrate [Clement, 2003]. Clement et al. reported that an increase in the nitrogen concentration led to an enhancement of the (102) and (101) orientations and a reduction of the preferential (002) orientation [Clement, 2003]. This is contrary to the observations made by Cheng et al. They observed an increase in preferred orientation (002) at high nitrogen concentrations and an increase in other structural orientations (001) at low concentrations [Cheng, 2003]. 
Additionally, since aluminum and AlN have different deposition rates, it is important that the $\mathrm{N}_{2}$ partial pressure be high enough to keep the target poisoned while reactive sputtering. This ensures that pure aluminum is not incorporated into the film [Boeshore, 2005].

\subsubsection{MAGNETRON POWER}

The discharge power has been observed to affect the deposition rate. An increase in the power has been reported to increase the deposition rate while a decrease in the power has been reported to decrease the deposition rate. The deposition rate has also been observed to affect the structure of the film [Iriarte, 2003; Hwang, 2002]. Experiments conducted by Cheng et al. show that the film growth shifts from the (002) preferred orientation to a less densely packed structure with a (101) orientation at high powers (500W) [Cheng, 2003]. They also reported that at low powers $(<100 \mathrm{~W})$, a smooth surface and small grain sizes were observed. This was attributed to the low energy and mobility of the adatoms at low rf powers [Cheng, 2003]. A high deposition rate may also negatively influence the quality of the films by increasing the surface roughness and tensile stress. This has been attributed to the reduction in ion/atom flux during ion assisted deposition [Iriarte, 2003].

We performed an experiment to determine the effect of varying the magnetron power on the film thickness, refractive index, and surface roughness. Prior to sputtering, silicon wafers were cleaned in a 5 minute bath in acetone followed by a 5 minute bath in methanol and were dried in $\mathrm{N}_{2}$ gas. The wafers were dipped in a 10:1 bath of de-ionized water to $49 \%$ J.T. Baker hydrofluoric acid for 5 minutes and were rinsed in a cascading bath of de-ionized water for 10 minutes. The wafers were immediately loaded and the sputtering chamber was pumped down to a base pressure of less than $5 \times 10^{-6}$ Torr before introducing the process gases. The nitrogen and argon flow rates were set to $27 \mathrm{sccm}$ and 3 sccm, respectively, and the working pressure was adjusted until it stabilized at 30 mTorr. An 8”, $99.999 \%$ pure aluminum target was pre-sputtered prior to depositing the films.

Four films were reactively sputtered for 50 minutes with magnetron powers ranging from 200 to $1100 \mathrm{~W}$ in increments of $300 \mathrm{~W}$. The magnetron power was limited to $1100 \mathrm{~W}$ due 
to excessive arcing at higher powers. The films were characterized using variable angle spectroscopic ellipsometry with incident angles of $55^{\circ}, 60^{\circ}$, and $65^{\circ}$ and a spectral range of $400 \mathrm{~nm}$ to $1000 \mathrm{~nm}$. The results are shown in Figures 2.5-2.7.

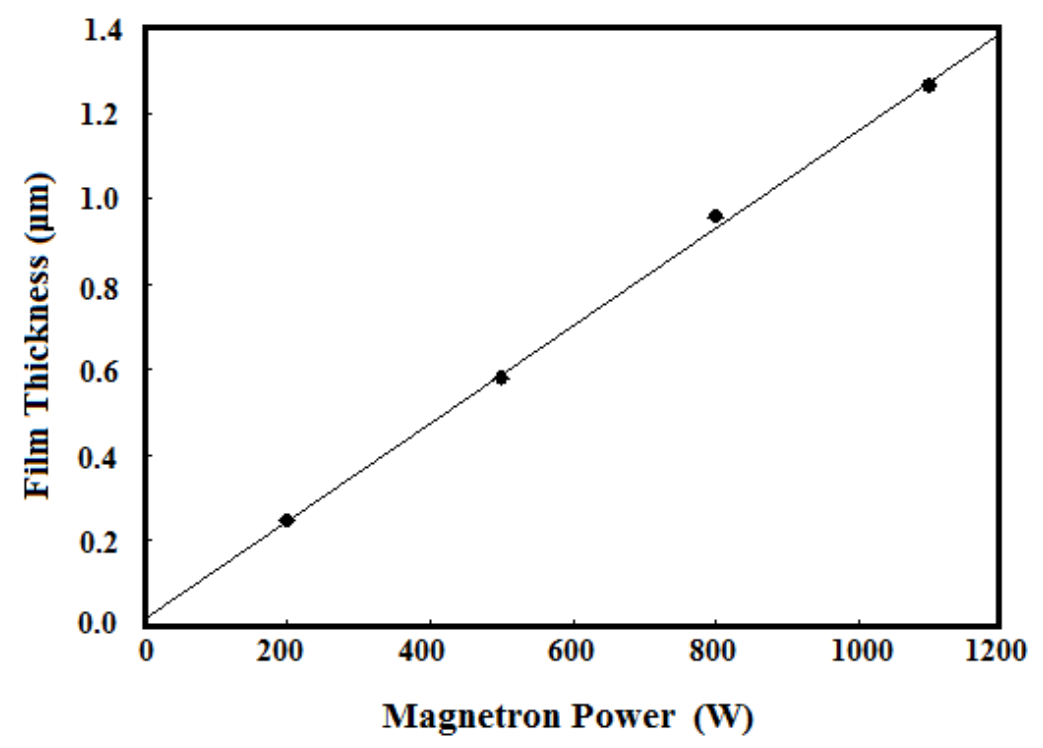

Figure 2.5. Dependence of the film thickness of reactively sputtered AlN films on magnetron power.

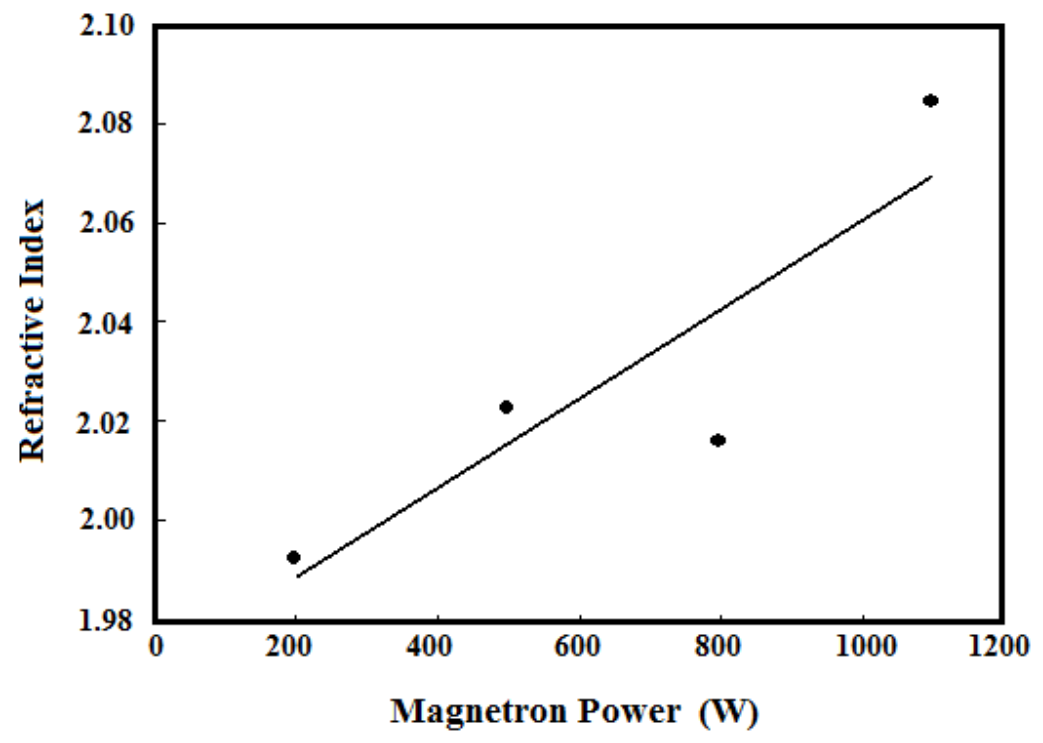

Figure 2.6. Dependence of the reactive index@588.947 nm of reactively sputtered AlN films on magnetron power. 




Figure 2.7. Dependence of the surface roughness of reactively sputtered AlN films on magnetron power.

As shown, the film thickness, refractive index, and surface roughness all were found to increase as the magnetron power was increased. The increase in film thickness (and thus surface roughness) agrees with the literature. In addition, it makes sense that films deposited at higher powers had slightly higher refractive indices indicating slightly better quality films. The magnetron power influences the average energy of adatoms on the surface of the deposited film. Thus, if the power is increased, the adatoms have more energy to migrate over the film forming larger crystalline regions.

\subsubsection{SUBSTRATE TEMPERATURE}

The substrate temperature during deposition has been reported to influence the energy available to the adatoms on the surface. The temperature can affect the film growth along the (002) preferred orientation in an equivalent manner to decreasing the substrate to target distance [Clement, 2003]. At higher temperatures $\left(500^{\circ} \mathrm{C}\right)$, the grains have been found to be more rounded than at lower temperatures $\left(250^{\circ} \mathrm{C}\right)$ [Cheng, 2003]. This was explained by the roughening effect caused by increased mobility of the adatoms at high temperatures [Cheng, 2003]. A correlation between the film density and the substrate 
material was noted by Fujiki et al. [Fujiki, 2000]. They noticed that the density of the film increased as the temperature decreased. This was attributed to the higher energy available to the adatoms during film growth [Fujiki, 2000]. A further increase of the substrate temperature has been found to have negative effects on the structure of the film. The negative effects include heightened processing costs, increased thermal stresses in the film, and an increase in the number of impurity inclusions [Iriarte, 2003].

\subsubsection{TARGET-TO-SUBSTRATE DiSTANCE}

The target-to-substrate distance has been reported to affect the energy delivered to the adatoms on the substrate surface. The effect on the AIN film has been observed to be similar to increasing the mean free path (see Section 2.2.3.2).

\subsubsection{SUBSTRATE BIAS}

A bias can be applied to the substrate to increase the kinetic energy translated to the substrate surface. By applying a moderate negative bias $(<-50 \mathrm{~V})$, positive ions will be accelerated into the growing film. Clement et al. suggested that the increase in the bias voltage enhances the (002) preferred orientation of AlN films [Clement, 2003]. It has also been observed that the surface roughness of the films can be lowered through increasing the surface diffusion. This can be accomplished by applying a bias voltage [Iriarte, 2003]. However, if an excessively high bias is applied, film damage due to ions with high kinetic energies can occur [Iriarte, 2003]. These findings show that a moderate substrate biasing may be optimal for producing high quality films.

Dubois et al. performed a study to determine the built-in stress and piezoelectric properties of reactively sputtered AlN films. AlN thin films with thicknesses of $0.5 \mu \mathrm{m}$ were reactively sputtered on hydrogen passivated silicon substrates coated with different metals including platinum. The authors noticed that the zero point on the residual stress depended on the substrate potential as shown in Figure 2.8. Additionally, the residual stress was a function of the $\mathrm{Ar} / \mathrm{N}_{2}$ flow rates, pressure, and $\mathrm{rf}$ power. It is interesting that these parameters did not influence the plasma in the same manner as the change in bias voltage did [Dubois, 2001]. 


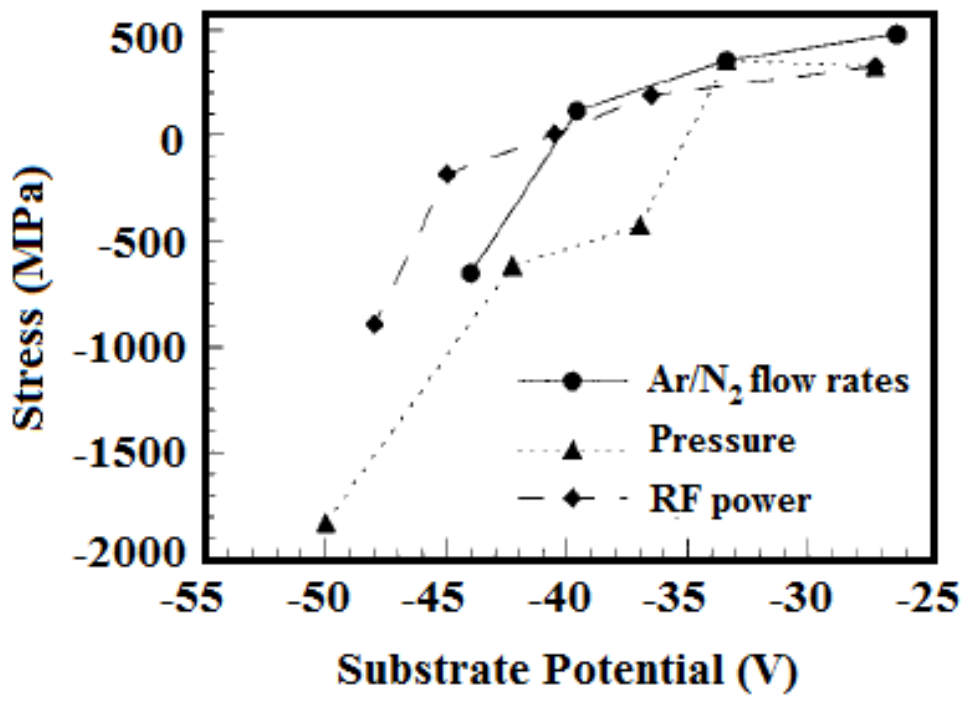

Figure 2.8. Dependence of the residual stress in reactively sputtered AlN films on substrate potential, flow rates, pressure, and magnetron power. After Dubois and Muralt [Dubois, 2001].

\subsubsection{SUMMARY}

Good quality aluminum nitride films have been deposited using reactive sputtering. The deposition parameters used by some research groups are summarized in Table 2.1. 
Table 2.1. Summary of deposition parameters reported in the literature for reactively sputtering AlN thin films.

\begin{tabular}{|c|c|c|c|c|c|c|}
\hline & $\begin{array}{l}\text { [Caliendo, } \\
\text { 1993] }\end{array}$ & $\begin{array}{l}\text { [Dogheche, } \\
\text { 1999] }\end{array}$ & $\begin{array}{l}\text { [Fujiki, } \\
\text { 2000] }\end{array}$ & $\begin{array}{l}\text { [Leung, } \\
\text { 2003] }\end{array}$ & $\begin{array}{l}\text { [Muhl, } \\
\text { 1997] }\end{array}$ & $\begin{array}{l}\text { [Cheng, } \\
\text { 2003] }\end{array}$ \\
\hline $\begin{array}{l}\text { Working } \\
\text { Pressure } \\
\text { (mTorr) }\end{array}$ & 1 to 8 & 900 & 6 to 37.5 & 5 & 3 & 1.4 to 20 \\
\hline $\begin{array}{l}\text { Gas } \\
\text { Composition } \\
\left(\% \mathrm{~N}_{2}\right)\end{array}$ & 50 to 100 & 30 & 100 & 80 & 50 to 90 & 0 to 100 \\
\hline $\begin{array}{l}\text { Magnetron } \\
\text { Power (W) }\end{array}$ & 500 & 300 & 100 & $\begin{array}{l}100 \text { to } \\
250\end{array}$ & 500 & $\begin{array}{c}100 \text { to } \\
500\end{array}$ \\
\hline $\begin{array}{l}\text { Substrate } \\
\text { Temperature } \\
\left({ }^{\circ} \mathrm{C}\right)\end{array}$ & 200 to 500 & 400 & $\sim 700$ & $\begin{array}{c}\sim 37 \text { to } \\
700\end{array}$ & 250 & $\begin{array}{c}100 \text { to } \\
500\end{array}$ \\
\hline $\begin{array}{l}\text { Target-to- } \\
\text { Substrate } \\
\text { Distance } \\
(\mathrm{mm})\end{array}$ & 55 & 50 & 30 to 65 & 100 & 5 & 140 \\
\hline $\begin{array}{l}\text { Substrate } \\
\text { Bias (V) }\end{array}$ & 150 to 200 & - & - & - & - & - \\
\hline
\end{tabular}

The optimal deposition parameters that we found for reactively sputtering aluminum nitride thin films using a CVC 610 DC magnetron sputter deposition system are provided in Table 2.2.

Table 2.2. Summary of optimal deposition parameters found for reactively sputtering AlN using a CVC 610 DC magnetron sputter deposition system.

\begin{tabular}{|c|c|c|c|}
\hline & 8" Al Target & 2" Al Target & $\begin{array}{c}2 " \mathrm{Al}_{0.97} \mathrm{Er}_{0.03} \\
\text { Target [Kabulski, } \\
\text { 2009] }\end{array}$ \\
\hline $\begin{array}{l}\text { Working Pressure } \\
\text { (mTorr) }\end{array}$ & 30 & 30 & 30 \\
\hline $\begin{array}{l}\mathrm{N}_{2} \text { Flow, Ar Flow } \\
(\mathrm{sccm})\end{array}$ & 27,3 & 27,3 & 27,3 \\
\hline Magnetron Power (W) & 500 & $100-200$ & $100-200$ \\
\hline $\begin{array}{l}\text { Substrate Temperature } \\
\left({ }^{\circ} \mathrm{C}\right)\end{array}$ & - & - & - \\
\hline Substrate Bias (V) & 0 & 0 & 0 \\
\hline
\end{tabular}




\subsection{ADDITIONAL CONSIDERATIONS}

Since reactive sputtering of AIN thins films is a complex process, several additional considerations are discussed in this section. These topics include film reproducibility, oxygen content, grain orientation, post-deposition annealing, and film thickness effects.

\subsubsection{REPRODUCIBILITY}

In order for meaningful relationships to be developed between the deposition parameters and the film properties, the sputtered AlN thin films must be reproducible. Muhl et al. performed a study to determine the reproducibility of the bandgap and refractive index of reactively sputtered AlN films. The authors found that the bandgap of these films varied by less than $2 \%$ and the index of refraction varied by $\pm 3 \%$ [Muhl, 1997]. Mahmood et al. also concluded that fluctuations in the deposition parameters (and thus the properties of deposited films) are unavoidable with RS [Mahmood, 2003]. Thus, there is an inherent limitation to the degree of reproducibility that can be achieved in reactively sputtered AlN films. However, the influence of external sources that increase the variation in the properties of sputtered films can be minimized through inclusion of a couple of procedures.

The first is the consistent use of an adequate cleaning method. Prior to sputtering, our group cleans the silicon wafers in a 5 minute bath in acetone followed by a 5 minute bath in methanol. The wafers and are then dried in $\mathrm{N}_{2}$ gas. The wafers are dipped in a 10:1 bath of de-ionized water to $49 \%$ J.T. Baker hydrofluoric acid for 5 minutes and are rinsed for 10 minutes in a cascading bath of de-ionized water. The hydrogen passivated silicon wafers are dried in $\mathrm{N}_{2}$ gas and are immediately loaded into the sputtering chamber.

The second is the consistent use of an adequate pre-sputtering procedure. This is important for the removal of native oxide and impurities on the aluminum target. Additionally, this ensures that the target is sufficiently poisoned prior to film deposition (see Section 2.2.3.1). A two-step pre-sputtering procedure was developed by our group that consists of 1) pre-sputtering the target for 10 minutes using pure argon gas followed by 2) pre-sputtering the target for 5 minutes using a mixture of nitrogen and argon gases. The 
consistent use of these two procedures has reduced the variation in the properties of our reactively sputtered films.

\subsubsection{OXYGEN CONTENT}

The unintentional incorporation of impurities such as oxygen in aluminum nitride films is typically undesirable. Oxygen has been shown to be detrimental to both the structural and the piezoelectric properties of polycrystalline AlN films [Engelmark, 2000; Iborra, 2004; Yu, 2005]. Additionally, oxygen incorporation can lower the thermal conductivity [McNeil, 1993; Mahmood, 2003]. However, the incorporation of some amount of oxygen is unavoidable due to the greater affinity of oxygen (compared with nitrogen) toward aluminum [Engelmark, 2000]. Furthermore, a background level of oxygen is always present in the sputtering chamber. Thus, almost all background levels of oxygen are incorporated into reactively sputtered AIN films [Engelmark, 2000] which can lead to a variety of intermediate phases referred to collectively as Aluminum OxyNitrides (AlON) [McNeil, 1993].

Various methods such as X-ray Photoelectric Spectroscopy (XPS), Rutherford Backscattering Spectroscopy (RBS), Auger Electron Spectroscopy, and Energy Dispersive Analysis of X-rays (EDAX) have been used to determine the concentration of oxygen in AlN films. Oxygen concentrations ranging from 0.5 to 15 atomic percent have be reported in the literature [Engelmark, 2000; Iborra, 2004; Muhl, 1997; Elmazria, 2003; Chowdhury, 1997; $\mathrm{Yu}, 2005]$. The intensity of the (002) X-ray diffraction peak has been shown to decrease while the FWHM has been shown to increase from $5.6^{\circ}$ to $11.4^{\circ}$ as the atomic concentration of oxygen in the film increases from $\sim 5 \%$ to $15 \%$ [Yu, 2005].

The variance in the oxygen content reported for reactively sputtered AIN films may be due to several factors. First, differences in the equipment and sputtering materials are

likely causes. Furthermore, differences in deposition parameters may affect the amount of oxygen included in the films since it is possible that some deposition conditions are more favorable for oxygen incorporation than others. In addition, the characterization methods used to determine the film compositions vary. For example, XPS can provide more accurate elemental composition information than EDAX resulting in lower uncertainty. 
However, since XPS is more surface sensitive, it may overestimate the oxygen concentration in the film if adventitious carbon contamination or surface oxidation is present.

\subsubsection{GRAIN ORIENTATION}

The measured piezoelectric response of an aluminum nitride thin film effectively provides the net piezoelectric response of the individual grains in the measurement area [Tonisch, 2006]. It has been reported that the highest piezoelectric response occurs when the grains are aligned in the same direction and have the same polarity [Xu, 2002; Iborra, 2004; Tonisch, 2006]. Thus, various efforts have been made to determine the deposition parameters that result in the highest degree of grain orientation. Chowdhury et al. found that lower working pressures and higher rf power result in stronger preferential orientations [Chowdhury, 1997]. However, Iborra et al. later reported that the crystalline quality of an AlN film (on its own) does not provide a good figure of merit for the piezoelectric response of the film. AlN films having high crystallinity were found to exhibit a poor piezoelectric response while AlN films having moderate crystallinity were found to exhibit almost ideal piezoelectric response [Iborra, 2005].

In addition to the deposition parameters used, it has been found that the crystallographic and piezoelectric properties are influenced by the substrate chosen [Dubois, 2001; Andrei, 2008]. Much work on the topic of sputtering AlN thin films on metal substrates has been reported in the literature [Dubois, 2001; Akiyama, 2004; Boeshore, 2005; Andrei, 2008]. Dubois et al. found that AIN films deposited on platinum,

aluminum, and titanium all exhibited columnar microstructures and a $c$-texture. They suggested that textured growth along the [001] direction was related to the quality of the metal substrate [Dubois, 2001]. Additionally, they found that the largest piezoelectric coefficients were obtained when platinum was used even though platinum had the largest lattice mismatch with AlN of the three metals investigated. Films deposited on platinum and aluminum were found to exhibit similar trends in their piezoelectric responses as the deposition parameters were varied while films deposited on titanium were found to be less dependent on the processing conditions [Dubois, 2001]. 
Akiyama et al. performed a comprehensive study to determine the influence of the substrate on the crystallinity and crystal orientation of AlN thin films. Fifteen metals were considered. They found that all of the AlN films deposited (regardless of the metal substrate used) exhibited some degree of $c$-axis orientation. However, it was noted that the XRD peak intensity of the AlN films varied with the metal used. AlN films deposited on metals having face centered cubic (fcc) lattices such as aluminum and platinum had a high degree of grain orientation while films deposited on metals having body center cubic (bcc) and hexagonal close packed (hcp) lattices did not. Thus, it was concluded that the substrate lattice type strongly influenced the growth mechanisms of reactively sputtered AIN films [Akiyama, 2004].

\subsubsection{Post-Deposition ANNEALING}

Post-deposition annealing has been reported to enhance the crystalline quality of reactively sputtered AlN films [Dogheche, 1999; Kar, 2005]. It was found that annealed films were also more transparent [Kar, 2005]. However, care must be taken if films are annealed in an oxygen containing environment. Since $\mathrm{N}_{2}$ is the strongest bonded diatomic molecule [Porowski, 2002] and since oxygen has a higher affinity for aluminum than nitrogen [Engelmark, 2000], annealing in high temperatures in the presence of oxygen can lead to film oxidation [Chowdhury, 1997].

We conducted a study to determine the oxidative behavior of reactively sputtered aluminum nitride films. A $0.18 \mu \mathrm{m}$ thick AlN film was reactively sputtered on a 3" $p$-type (100) silicon substrate (deposition parameters similar to those in Table 2.2). The wafer was cut into sixteen pieces and the pieces were separated into eight pairs by size. The stoichiometry of the AlN films were determined prior to annealing using EDAX. The two pieces comprising each pair were stacked on top of each other such that the AlN coated sides faced each other. The pairs were annealed at a temperature in the range of $750^{\circ} \mathrm{C}$ to $1100^{\circ} \mathrm{C}$ (in $50^{\circ} \mathrm{C}$ intervals) in an oxygen containing environment. The stoichiometries of the annealed films were determined using EDAX. Figure 2.9 shows that the threshold oxidation temperature for reactively sputtered AlN films is approximately $800^{\circ} \mathrm{C}$. 


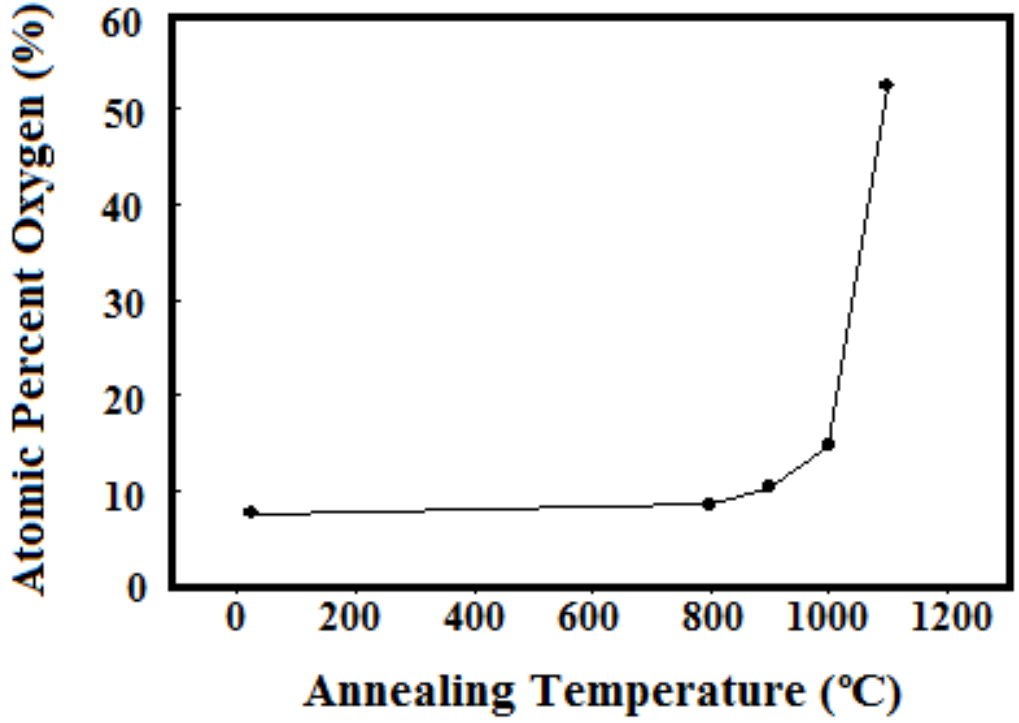

Figure 2.9. Oxidation of reactively sputtered AlN thin films. After Farrell et al. [Farrell, 2008].

Thus, if a post-deposition annealing step is employed, it should be done so in a nitrogen environment. Typical temperatures and times for this step are 600 to $800^{\circ} \mathrm{C}$ and $\sim 30$ minutes, respectively [Kar, 2005].

\subsubsection{FILM THICKNESS EFFECTS}

The material properties of sputtered AlN films have been found to be dependent on the film thickness. Models for the deposition process were discussed in Section 2.2.2. In this section, the effect of thickness on breakdown strength, surface roughness, crystalline quality, internal stress, and piezoelectric response is addressed.

\subsubsection{BREAKDOWN STRENGTH}

One of the main advantages of aluminum nitride has over materials such as zinc oxide is its high breakdown strength [Akiyama, 2004; Martin, 2004]. The breakdown strength for reactively sputtered AlN has been reported to be between 3 and $12 \mathrm{MV} \mathrm{cm}^{-1}$ [Liufu, 1998; Adam, 2000]. However, the breakdown strength has been found to vary with film thickness. Liufu et al. studied the dependence of the breakdown strength of reactively sputtered AlN films as a function of film thickness. They reported that the breakdown 
strength decreased from $\sim 3.8 \mathrm{MV} \mathrm{cm}^{-1}$ to $\sim 3.1 \mathrm{MV} \mathrm{cm}^{-1}$ as the film thickness increased from $\sim 0.3 \mu \mathrm{m}$ to $\sim 2.6 \mu \mathrm{m}$ as shown in Figure 2.10 [Liufu, 1998].

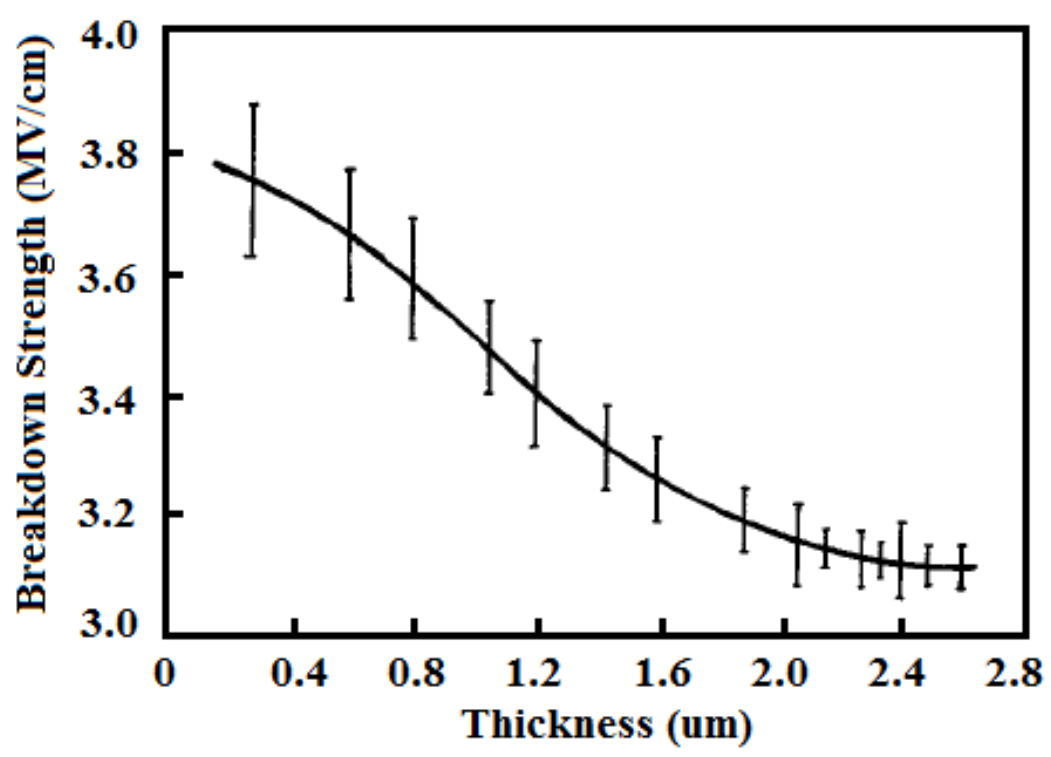

Figure 2.10. Dependence of the breakdown strength of reactively sputtered AlN films on film thickness. After Liufu and Cao [Liufu, 1998].

\subsubsection{SURFACE ROUGHNESS}

Films with low surface roughness are especially important to the performance of surface acoustic wave devices. A low surface roughness minimizes the SAW energy propagation loss and thus reduces the insertion loss of the device [Assouar, 2004; Fansler, 2008]. However, the surface roughness has been found to vary with film thickness. Martin et al. studied the dependence of the surface roughness of reactively sputtered AlN films as a function of thickness. The authors found that although the magnitude of surface roughness increased with increased thickness, the fraction of surface roughness to film thickness decreased. The surface roughness was found to increase from $\sim 0.4 \mathrm{~nm}$ to $\sim 1.8$ $\mathrm{nm}$ as the film thickness increased from $35 \mathrm{~nm}$ to $2 \mu \mathrm{m}$ as shown in Figure 2.11 [Martin, 2004]. This agrees with the results that our group found in Section 2.2.3.4. 


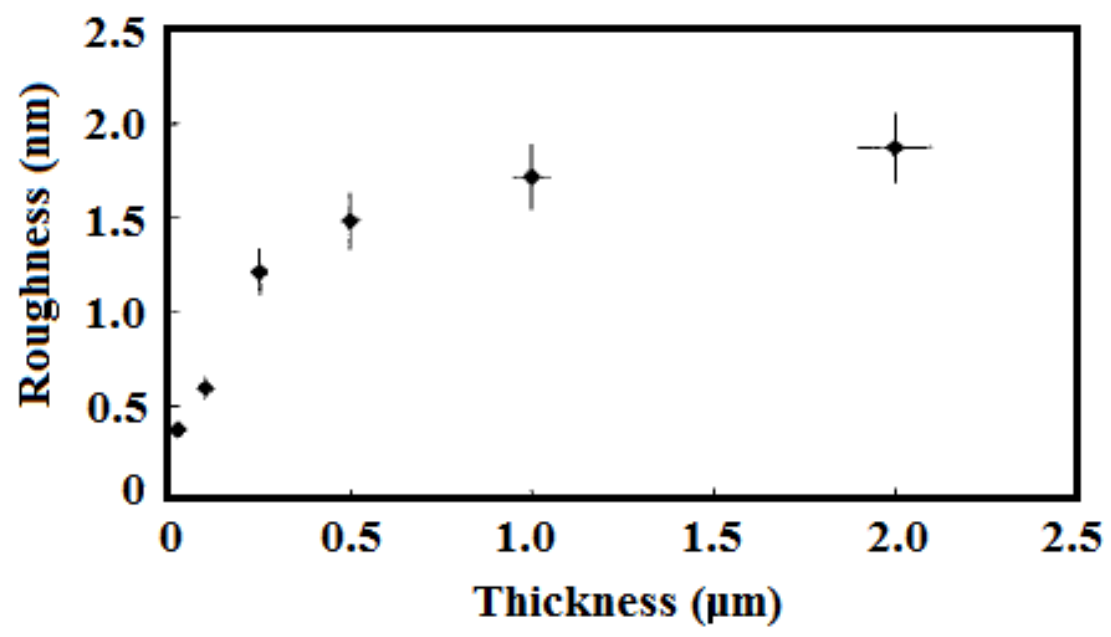

Figure 2.11. Dependence of the roughness of reactively sputtered AlN films on film thickness. After Martin et al. [Martin, 2004].

\subsubsection{INTERNAL STRESS}

The high residual stress frequently present in polycrystalline AlN thin films can complicate their integration in MEMS devices. Studies on this topic have shown that the residual stress is a function of film thickness. Jergal et al. found that very thin films $(<50$ $\mathrm{nm}$ ) have a high tensile in-plane stress. The high stress was attributed to lattice distortions resulting from the small size of the grains comprising the films. Additionally, they found that the tensile in-plane was relieved as the film thickness was increased [Jergal, 2003]. Martin et al. also studied the dependence of the residual stress in reactively sputtered AlN films as a function of thickness. They found that the stress smoothly changed from compressive to tensile as the film thickness increased from $35 \mathrm{~nm}$ to $2 \mu \mathrm{m}$ as shown in Figure 2.12. The maximum residual stress was present in very thin films while the minimum was present for films with a thickness of $1 \mu \mathrm{m}$ [Martin, 2004]. It can also be observed that the stress in the films began to saturate as the film thickness increased. 


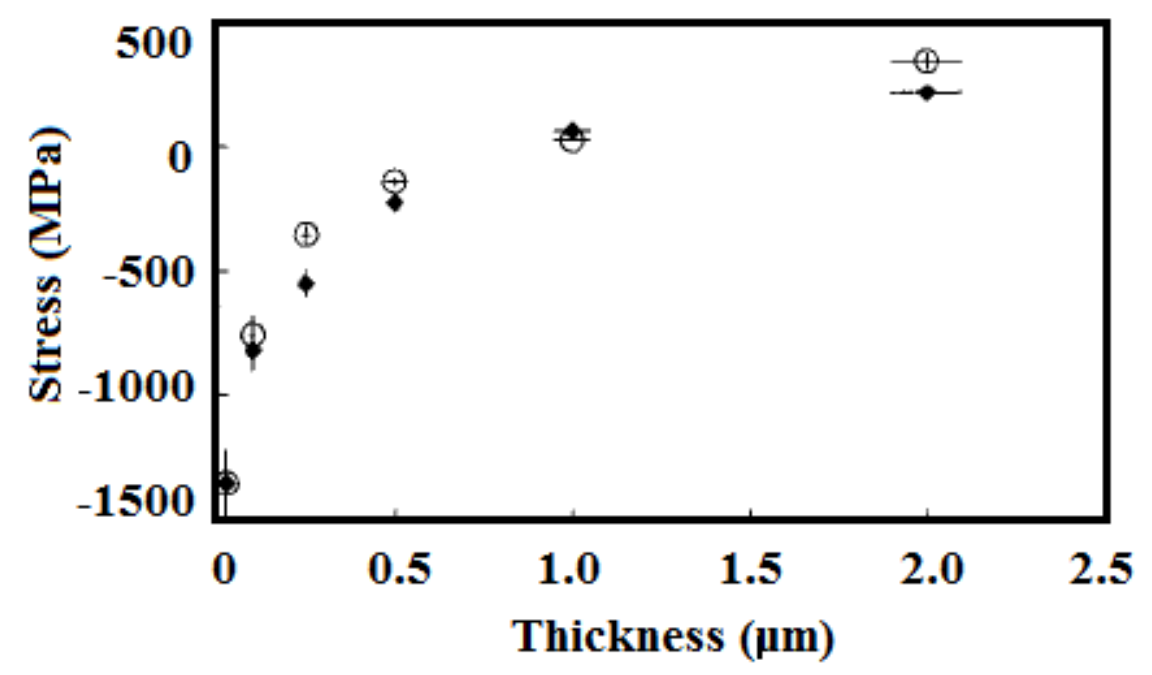

Figure 2.12. Dependence of the residual stress of reactively sputtered AlN films on film thickness. After Martin et al. [Martin, 2004].

The residual stress is commonly determined by measuring the radius of curvature of the substrate prior to and after the deposition of the film [Olivares, 2005]. Stoney's equation is used to calculate the stress in the film from the change in the radius of curvature when the substrate thickness, $t_{s}$, film thickness, $t_{f}$, Young's modulus of the substrate, $E_{s}$, and Poisson's ratio of the substrate, $v_{s}$, are known as given in (2.2) [Campbell, 2001; Dubois, 2001].

$$
\sigma=\frac{E_{s}}{6\left(1-v_{s}\right)} \frac{t_{s}^{2}}{t_{f}}\left[\frac{1}{R}-\frac{1}{R_{0}}\right]
$$

\subsubsection{CRYSTALLINITY}

The effect of the crystallinity and grain orientation on the piezoelectric response of reactively sputtered AlN thin films was discussed in Section 2.3.3. Reactively sputtered films with thicknesses of $35 \mathrm{~nm}$ to $2 \mu \mathrm{m}$ were all reported to have $c$-axis orientation [Martin, 2004]. However, crystallinity has been found to improve with film thickness. Jergal et al. found that although the (002) texture improved with film thickness, (002) texture is not directly proportional to the film thickness [Jergal, 2003]. 


\subsubsection{Piezoelectric ReSPONSE}

One of the most important characteristics of an actuator layer for MEMS applications is its piezoelectric response [Iborra, 2004]. Thus, maximizing the response of piezoelectric thin films has been the subject of much research. The piezoelectric response of reactively sputtered aluminum nitride films has been found to increase with film thickness. Martin et al. found that $2 \mu \mathrm{m}$ AIN films had the highest piezoelectric response of films within a thickness range of $35 \mathrm{~nm}$ to $2 \mu \mathrm{m}$ [Martin, 2004]. We have also observed that films with thicknesses below $0.2 \mu \mathrm{m}$ have negligible piezoelectric responses as shown in Figure 2.13 [Kabulski, 2009].

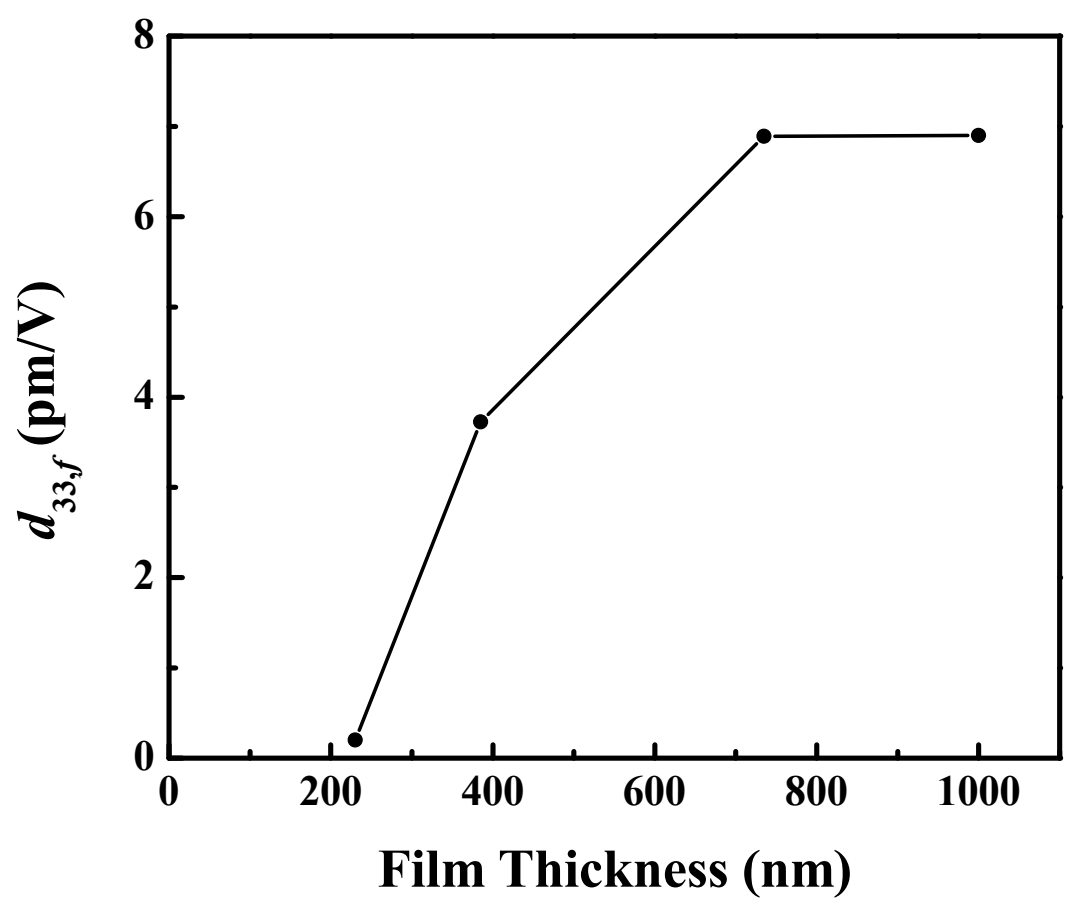

Figure 2.13. Dependence of the piezoelectric response of reactively sputtered AlN films on film thickness. After Kabulski et al. [Kabulski, 2009].

\subsection{SUMMARY}

High quality thin films of aluminum nitride have been deposited using reactive sputtering. This technique has proven to be a competitive technique for the inexpensive, low temperature deposition of AlN. Since RS is plasma based, it is inherently complex and 
can be hard to optimize. In this work, the deposition parameters were optimized for crystallinity and piezoelectric response. It was found that the consistent use of adequate cleaning and pre-sputtering procedures lead to the most reproducible depositions of AlN thin films. However, the oxygen contents in our films were relatively high (see Section 3.2.2). Finally, we determined that our reactively sputtered AIN films are piezoelectrically active for films having thicknesses greater than $200 \mathrm{~nm}$. We believe that this can be explained in terms of the quality of the films as a function of film thickness. 


\section{H A P T E R 3 : T H I N F I L M C H A R A C T E R I Z A T I O N}

\subsection{ChaRACTERIZATION TECHNIQUES}

In order to optimize the deposition parameters discussed in Chapter 2, the films must be characterized. Thus, the deposition and characterization techniques are often performed sequentially. In this work, a variety of characterization techniques including $\mathrm{VASE}^{\circledR}, \mathrm{AFM}, \mathrm{LDV}, \mathrm{XPS}$, and $\mathrm{C}-\mathrm{V}$ measurements were used to characterize aluminum nitride and UltraNanoCrystalline Diamond (UNCD) thin films. Case studies are presented in Section 3.2.

\subsubsection{AFM}

Atomic Force Microscopy, or AFM, is a technique used to measure extremely small surface features. This technique involves scanning the surface of a sample with a very sharp tip mounted on the end of a cantilever. A laser is shone on the cantilever and the reflected beam is directed onto a position detector as shown in Figure 3.1.



Figure 3.1. Schematic of AFM beam deflection measurement system [Nanoscience Instruments, 2008].

AFM offers several modes of operation including tapping mode, contact mode, Lateral Force Microscopy (LFM), Electrostatic Force Microscopy (EFM), Magnetic Force Microscopy (MFM), and Scanning Kelvin Probe Microscopy (SKPM), among others. Depending on the mode used, information about the sample's topography and/or material properties can be obtained. 


\subsubsection{TAPPING MODE AFM}

Tapping mode AFM can be used to simultaneously measure the topography and phase of a sample. In this mode, the cantilever is harmonically oscillated. The tip deflection, $\delta_{1}$, of the cantilever when it is far from the sample can be expressed as (3.1) where $\omega$ is the frequency, $A_{1}$ is the amplitude, and $\phi_{1}$ is the phase.

$$
\delta_{1}(t)=A_{1} \cos \left(\omega t+\phi_{1}\right)
$$

The amplitude and phase of the vibrating cantilever change as the cantilever is brought close to the sample from far away. The tip deflection, $\delta_{2}$, of the cantilever when it is close to the sample can be expressed as (3.2) where $\omega$ is the driving frequency, $A_{2}$ is the amplitude, and $\phi_{2}$ is the phase.

$$
\delta_{2}(t)=A_{2} \cos \left(\omega t+\phi_{2}\right)
$$

The change in the tip deflection in the two cases is due to the van der Waals force interaction between the tip and the sample when they are brought close to each other. This interaction reduces the amplitude and shifts the phase of the vibrating cantilever. The amplitude attenuation, $\alpha$, can be expressed as (3.3) and the phase shift, $\Delta \phi$, can be expressed as (3.4).

$$
\begin{aligned}
& \alpha=\frac{A_{2}}{A_{1}} \\
& \Delta \phi=\phi_{2}-\phi_{1}
\end{aligned}
$$

The change in phase between the driven oscillations and the detected oscillations can provide information on the sample's properties such as viscoelasticity, friction 
coefficient, and stiffness. In this work, all AFM measurements were performed using tapping mode.

\subsubsection{VAN DER WAALS FORCE}

The van der Waals force between two atoms located at a distance $r$ from each other can be approximated using the Lennard-Jones 6-12 potential [Ashcroft, 1976]. The Lennard-Jones 6-12 potential can be expressed as (3.5) where $\sigma=(B / A)^{1 / 6}$, $\varepsilon=A^{2} / 4 B$, and $A$ and $B$ are fitting constants.

$$
\phi(r)=4 \varepsilon\left[\left(\frac{\sigma}{r}\right)^{12}-\left(\frac{\sigma}{r}\right)^{6}\right]
$$

Thus, the Lennard-Jones 6-12 potential consists of a long-distance attractive force $\left(1 / r^{6}\right)$ and a short-distance repulsive force $\left(1 / r^{12}\right)$. A plot of the Lennard-Jones 6-12 is shown in Figure 3.2.

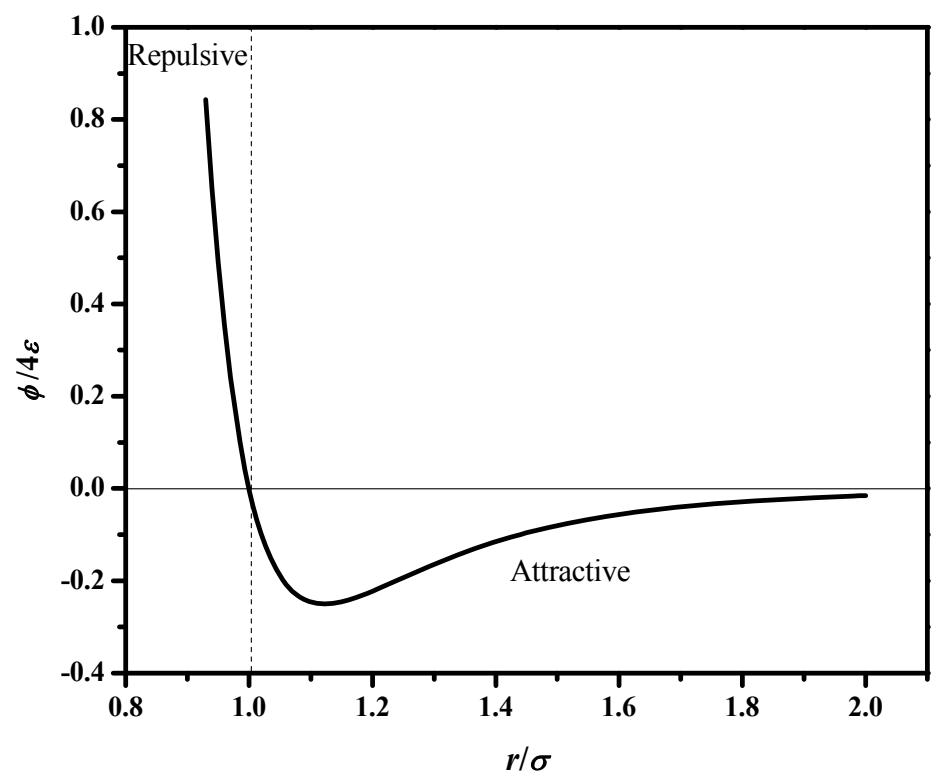

Figure 3.2. Plot of the Lennard-Jones 6-12 potential. 


\subsubsection{EXAMPLES FROM LITERATURE}

AFM is commonly used to measure the surface roughness of thin AlN films, among others [Mortet, 2002; Iriarte, 2003; Assouar, 2004]. Assouar et al. deposited $2 \mu \mathrm{m}$ thick aluminum nitride films using reactive sputtering for use in surface acoustic wave devices. The root-mean-squared (rms) roughness was measured to be $6.15 \mathrm{~nm}$ over a $1 \mu \mathrm{m}$ x $1 \mu \mathrm{m}$ scan and $5.86 \mathrm{~nm}$ over a $5 \mu \mathrm{m} \times 5 \mu \mathrm{m}$ scan using AFM. The 2- $d$ and 3-d topography images are shown in Figure 3.3. The low surface roughness was suggested to be one of the reasons that SAW devices using these films exhibited low acoustic losses [Assouar, 2004].

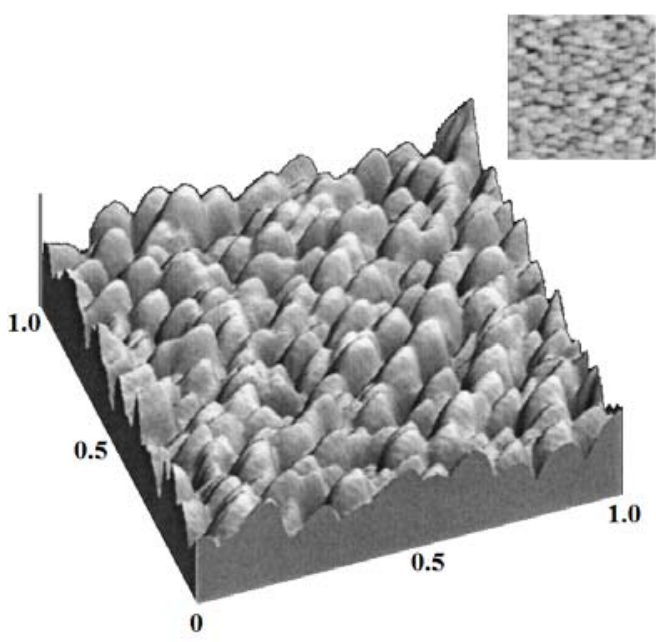

(a)

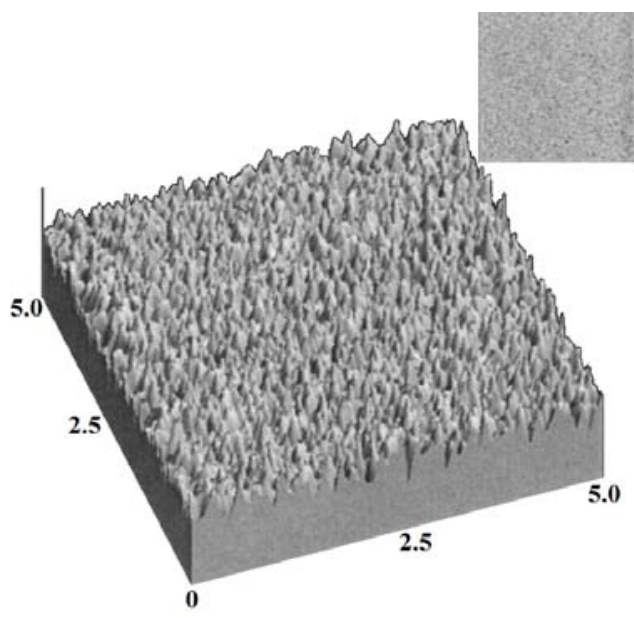

(b)

Figure 3.3. Topography of $2 \mu \mathrm{m}$ thick reactively sputtered AlN film. (a) $1 \mu \mathrm{m} \times 1 \mu \mathrm{m}$ scan and (b) $5 \mu \mathrm{m} \times 5$ um scan. After Assouar et al. [Assouar, 2004].

Mortet et al. deposited $100 \mu \mathrm{m}$ thick polycrystalline diamond films and $1 \mu \mathrm{m}$ thick aluminum nitride films using Plasma Enhanced Chemical Vapor Deposition (PECVD) and reactive sputtering, respectively. The rms roughness was measured to be $<15 \mathrm{~nm}$ for the diamond films and $<1 \mathrm{~nm}$ for the AlN films using AFM. The 3- $d$ topography images are shown in Figure 3.4. 


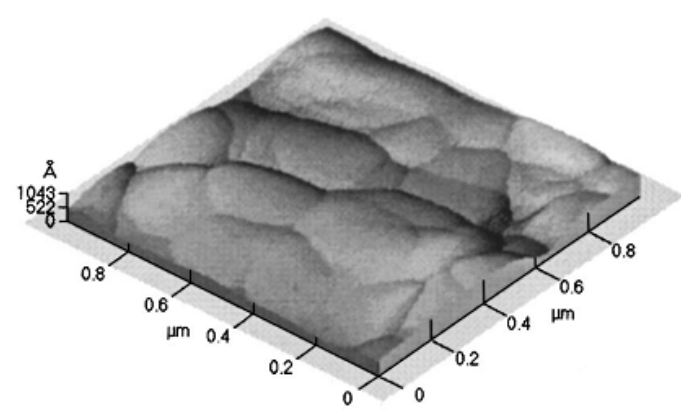

(a)

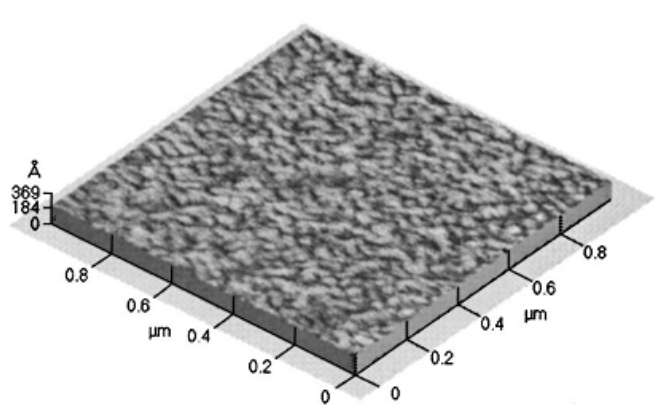

(b)

Figure 3.4. Topography of (a) $100 \mu \mathrm{m}$ thick polycrystalline diamond film and (b) $1 \mu \mathrm{m}$ thick reactively sputtered AlN film on silicon [Mortet, 2003].

\subsubsection{LDV}

Laser Doppler Vibrometry (LDV) is a technique used to determine the acceleration, velocity, and displacement of surface vibrations. Typically, a single laser beam is split into two beams. At the optical splitter, both beams have the same phase. One beam, termed the "reference beam", is sent along an optical path with a fixed distance. The other beam, termed the "measurement beam", is sent along an optical path whose total distance varies as a function of the surface vibrations. Both beams are reflected back to a detector where their phase difference is measured as shown in Figure 3.5. 


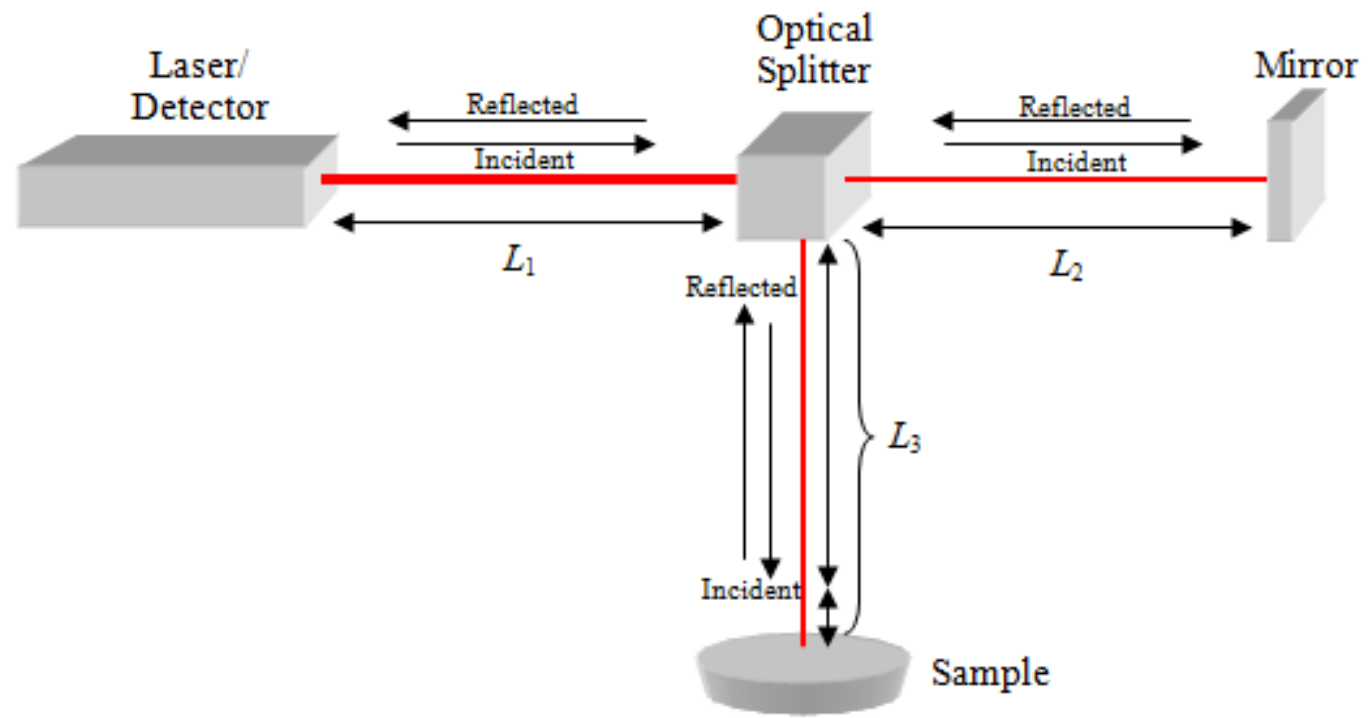

Figure 3.5. Schematic of $L D V$ setup.

The reference beam path length, $L_{\text {ref }}$, and measurement beam path length, $L_{\text {meas }}$, can be expressed as (3.6) and (3.7), respectively.

$$
\begin{aligned}
& L_{\text {ref }}=2\left(L_{1}+L_{2}\right) \\
& L_{\text {meas }}=2\left(L_{1}+L_{3}\right)=2\left(L_{1}+\left(L_{2}+\Delta L\right)\right)
\end{aligned}
$$

During alignment, the path lengths are set to be approximately equal (i.e. $\Delta L \approx 0$ ). When the sample vibrates, the measurement path length will change as function of the sample vibration as expressed in (3.8).

$$
L_{\text {meas }}(t)=2\left(L_{1}+L_{3}(t)\right)=2\left(L_{1}+L_{2}+\Delta L(t)\right)
$$

Thus, the measurement path length will be a function of time and will lead to a time-dependent phase difference, $\Delta \phi(t)$, between the two beams producing a phasedependent interference intensity, $I(\Delta \phi(t))$. The phase difference and interference intensity 
can be expressed as (3.9) and (3.10), respectively, where $\lambda$ is the wavelength of the laser and $I_{\max }$ is the maximum interference intensity [Yao, 2004].

$$
\begin{aligned}
& \Delta \phi(t)=\frac{2 \pi \Delta L(t)}{\lambda} \\
& I(\Delta \phi(t))=\frac{1}{2} I_{\text {max }}(1+\cos \Delta \phi(t))
\end{aligned}
$$

The interference fringe pattern also moves on the detector as the sample vibrates allowing the displacement to be determined. Additionally, the measurement beam is subjected to a small Doppler frequency shift, $f_{D}$, due to the moving sample surface which allows the velocity, $v$, to be determined. The expression for the Doppler frequency shift is given in (3.11) [Yao, 2004].

$$
f_{D}=\frac{2|v|}{\lambda}
$$

\subsubsection{EFFECTIVE Piezoelectric Coefficient}

Typically, LDV is used to measure the piezoelectric response of clamped films. A clamped film is one that is constrained in one or more dimensions by its physical surroundings. All reactively sputtered AIN thin films are clamped to their substrates. Figure 3.6 shows a clamped AIN film on an electrically conducting silicon substrate with a top and bottom metal contact. 


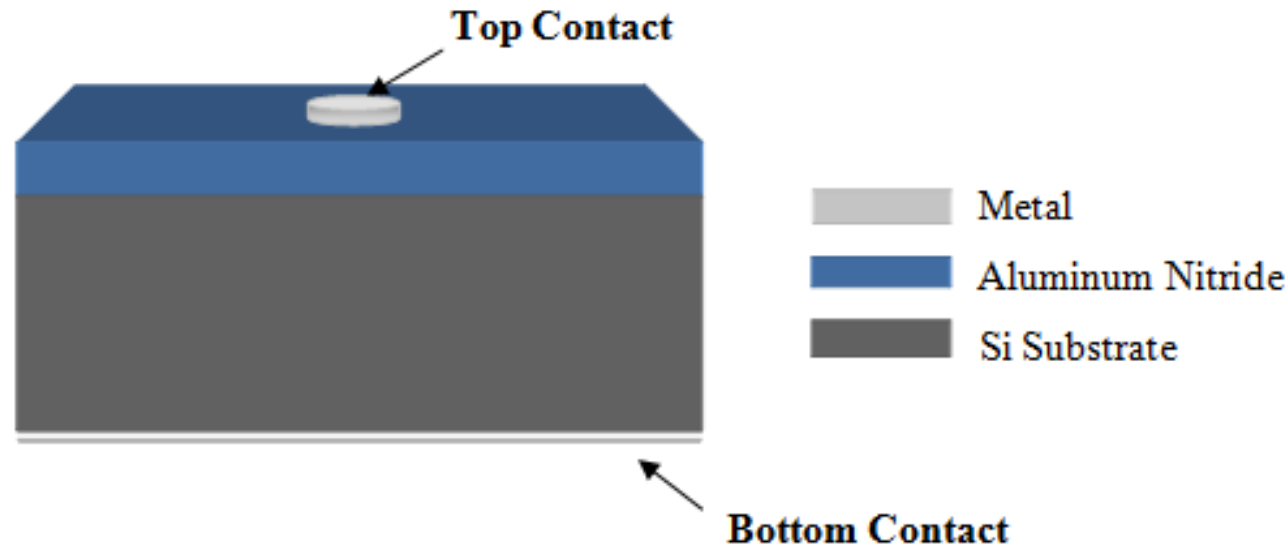

Figure 3.6. Schematic of a Metal-Insulator-Semiconductor (MIS) structure.

The contacts shown in Figure 3.6 are used to apply an ac voltage across the aluminum nitride film. The effect of the metal used for the contact is discussed in Section 3.1.2.2. The displacement of the film in the direction normal to the film surface can be expressed as (3.12) where $\delta_{f i m}$ and $\delta_{\text {sub }}$ are the deflections of the film and substrate, respectively [Yao, 2004].

$$
\delta=\delta_{\text {film }}-\delta_{\text {sub }}
$$

To calculate the effective longitudinal piezoelectric constant, $d_{33}$, assumptions have to be made about how the film is constrained. Yao et al. discussed the two extremes, namely, free in-plane constraint and extremely high in-plane constraint. In the free inplane constraint case, the longitudinal piezoelectric constant is calculated based on an assumption that the film experiences zero in-plane constraint. This case can be expressed in equation form as (3.13) where $\delta$ is given by (3.12) and $V$ is the applied voltage [Yao, 2004].

$$
d_{33, f}=\frac{\delta}{V}
$$


In the extremely high in-plane constraint case, the longitudinal piezoelectric constant is calculated based on an assumption that the film experiences zero in-plane strain. This case can be expressed in equation form as (3.14) where $\delta$ is given by (3.12), $V$ is the applied voltage, $d_{31}$ is the transverse piezoelectric constant, and $s^{\mathrm{E}}$ are components of the compliance tensor at a constant electric field [Yao, 2004].

$$
d_{33, c}=\frac{\delta}{V}+\frac{2 d_{31} s_{13}^{E}}{s_{11}^{E}+s_{12}^{E}}
$$

Yao et al. compared the results from using the two extreme cases with the results of using Finite Element Analysis (FEA). They found that in comparison to the FEA results, the first case underestimated the longitudinal piezoelectric coefficient while the second case overestimated it [Yao, 2004]. In addition, the compliance coefficients of the film must be known prior to calculating the extremely high in-plane constraint case [Dubois, 1999]. Thus, in this work the zero in-plane constraint case was used when calculating the effective piezoelectric coefficient.

\subsubsection{EFFeCt Of CONTACT Metal}

We found that effective longitudinal piezoelectric coefficient, $d_{33, f}$, was dependent on the work function of the metal used [Harman, 2008]. Aluminum nitride films with a nominal thickness of $1.1 \mu \mathrm{m}$ were reactively sputtered on double side polished $p$-type (100) silicon substrates with resistivities between 1 and $20 \Omega \mathrm{cm}$. An aluminum backside contact was sputtered on the side opposite to the AIN side. Circular metal contacts of aluminum, gold, titanium, and platinum with radii of $400 \mu \mathrm{m}$ were deposited onto the AIN film. The resulting configuration was similar to the one shown in Figure 3.6. A square wave voltage with a peak-to-peak voltage varying from $5 \mathrm{~V}$ to $40 \mathrm{~V}$ was applied between the top and bottom contacts. The resulting displacements are shown in Figure 3.7 [Harman, 2008]. 




Figure 3.7. Displacement of a $1.1 \mu \mathrm{m}$ thick reactively sputtered AlN film with various metal top contacts. After Harman et al. [Harman, 2008].

As shown in Figure 3.7, higher displacement values corresponded to higher metal work functions. Thus, platinum contacts were mostly used in this work since they provided the highest piezoelectric response.

\subsubsection{VibRATIONAL MODES}

Yao et al. investigated the vibrational modes produced when exciting a piezoelectric material with an ac signal using circular contacts. They found that some films exhibited the expected regular vibrational modes while other films exhibited distorted ones as shown in Figure 3.8. 


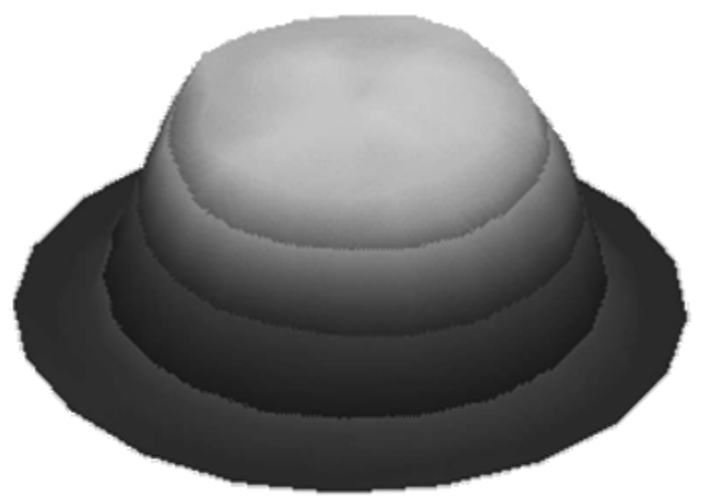

(a)

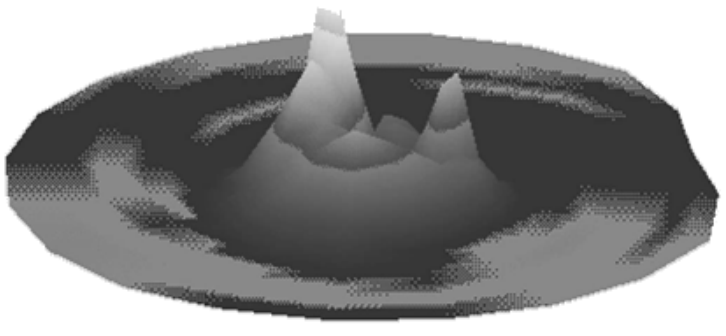

(b)

Figure 3.8. Vibrational modes of piezoelectric films with circular contacts. (a) regular modal shape and (b) distorted modal shape. After Yao et al. [Yao, 2004].

Yao et al. suggested that the distorted modalities displayed by some films were due to defects or unstable clamping [Yao, 2004]. Our group has also noticed similar modalities in AlN samples with $400 \mu \mathrm{m}$ in radius circular metal contacts [Farrell, 2009]. It is also important to note that the displacement of the piezoelectric film is a function of position. Thus, in this work the center of the contact is probed when measuring the piezoelectric response of circular contacts.

\subsubsection{ELLIPSOMETRY}

Ellipsometry is a technique used to measure the optical properties of a material. Light with a known polarization state is shone on a material and the polarization state of the reflected/transmitted light is measured. In this work, reflection measurements were performed at three angles using variable angle spectroscopic methods (see Section 3.1.3.1). A schematic illustrating a typical reflection ellipsometry setup is shown in Figure 3.9. 


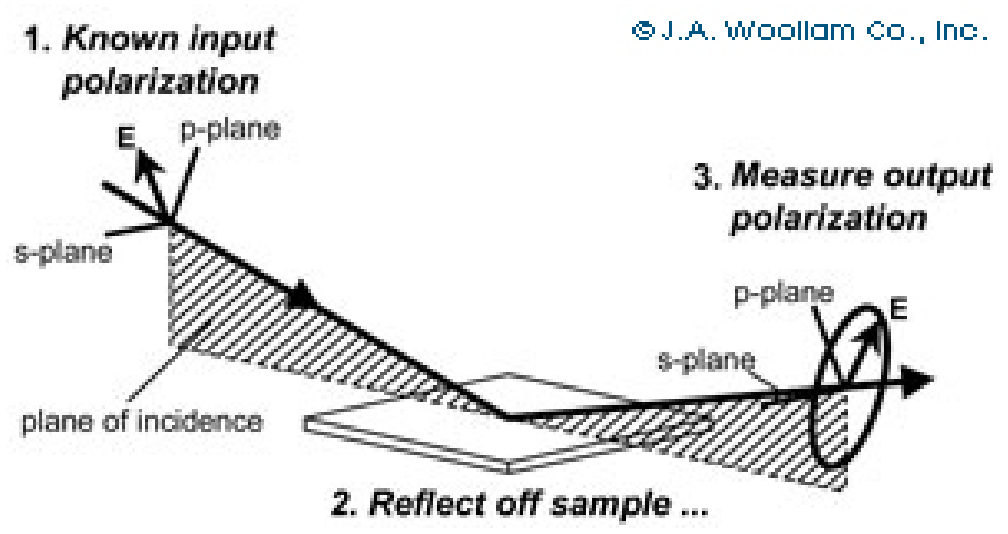

Figure 3.9. Reflection ellipsometry setup [Woollam, 2001].

Ellipsometry determines the parameters $\psi$ and $\Delta$ by measuring the complex ratio of the $p$ - and $s$ - polarized components, $R_{P} / R_{S}$, as given in (3.15) [Jones, 1999].

$$
\tan (\Psi) e^{\Delta i}=\frac{R_{P}}{R_{S}}
$$

Since ellipsometry measures a ratio, the results should be accurate and reproducible without the use of a reference material [Woollam, 2000]. The parameters $\psi$ and $\Delta$ are extracted using (3.15). The parameters are then used to fit the measured spectra to the model generated spectra using the process shown in Figure 3.10. 


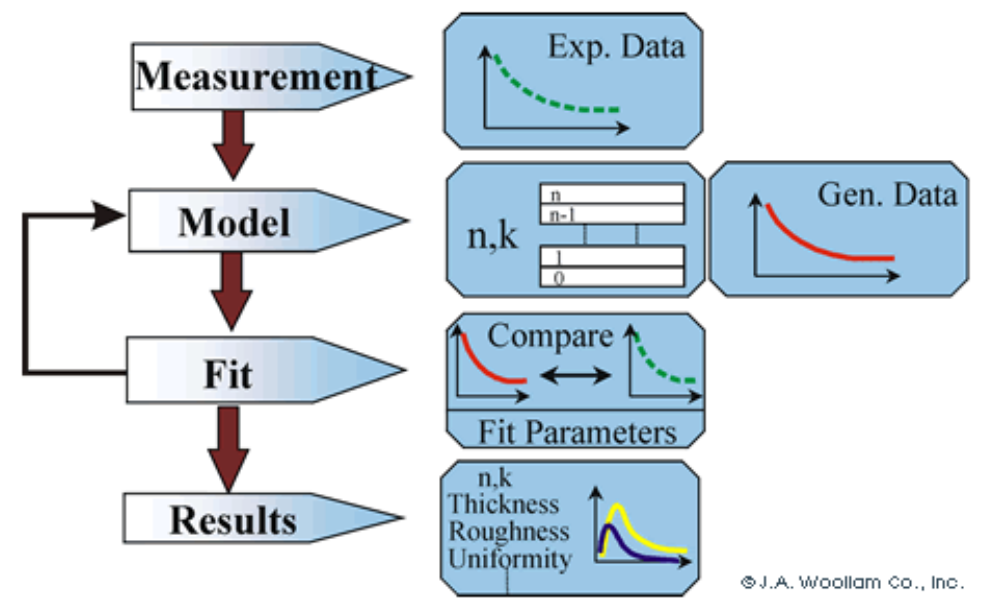

Figure 3.10. Ellipsometric process flow for determining the optical properties of a material [Woollam, 2001].

As shown in Figure 3.10, the construction of a reasonable model of the material is imperative for accurately determining the material's optical properties. In this work, the Cauchy dispersion model was used for the AlN layer. This model is well suited for dielectrics and semiconductors that are transparent over the measured spectral range [Woollam, 2001]. The real and imaginary components of the complex refractive index used in the Cauchy dispersion model can be expressed as functions of wavelength, $\lambda$, and fitting parameters, $A_{n}, B_{n}, C_{n}, \ldots, A_{k}, B_{k}$. The expressions for the real and imaginary components of the complex refractive index are given in (3.16) and (3.17), respectively [Woollam, 2001].

$$
\begin{aligned}
& n=A_{n}+\frac{B_{n}}{\lambda^{2}}+\frac{C_{n}}{\lambda^{4}}+\ldots \\
& k=A_{k} e^{-B_{k} \lambda}
\end{aligned}
$$

One figure of merit for determining how well the constructed model fits the measured data is the Mean Square Error (MSE). Ideally, the model will fit the measured data perfectly leading to a zero MSE. However, in practice, this is rarely the case. The 
MSE can be expressed as (3.18) where $N$ is the number of wavelengths, $M$ is the number of unknowns in the model, and $\sigma$ is the standard deviation [Woollam, 2001].

$$
M S E=\frac{1}{2 N-M} \sum_{i=1}^{N}\left[\left(\frac{\psi_{i}^{\bmod }-\psi_{i}^{\exp }}{\sigma_{\psi}^{\exp }}\right)^{2}+\left(\frac{\Delta_{i}^{\bmod }-\Delta_{i}^{\exp }}{\sigma_{\Delta}^{\exp }}\right)^{2}\right]
$$

In general, the $M S E / t_{f i m}$ should be less than $10 \%$ before the optical properties provided by the fit can be used with any degree of confidence.

\subsubsection{1 $\operatorname{VASE}^{\circledR}$}

Variable-Angle Spectroscopic Ellipsometry ${ }^{\circledR}\left(\mathrm{VASE}^{\circledR}\right)$ determines the parameters $\psi$ and $\Delta$ at multiple angles. Since $\operatorname{VASE}^{\circledR}$ is typically performed over a range of wavelengths, the thickness and optical constants of a material can be determined in a single ellipsometry measurement [Wang, 2005]. Typically, the measurements are performed around the Brewster angle of the material. The Brewster angle is defined (in ellipsometric terms) as the angle of incidence, $\theta$, in which the phase parameter, $\Delta$, is equal to $90^{\circ}$ [Woollam, 2001]. This angle leads to a purely imaginary ratio of $R_{P} / R_{S}$ by considering (3.15). From a more physical point of view, the Brewster angle is simply the angle of incidence where the reflected and transmitted rays are normal to each other. This relation can be expressed as (3.19) where $n_{1}$ is the refractive index of medium 1 and $n_{2}$ is the refractive index of medium 2 .

$$
\theta_{B}=\tan ^{-1}\left(\frac{n_{2}}{n_{1}}\right)
$$

It is recommended that the ellipsometry measurements be taken on or around the Brewster angle in order to improve the sensitivity of the measurement [Woollam, 2001]. For the ellipsometry measurements, medium 1 is air $\left(n_{1} \approx 1\right)$ and medium 2 is $\operatorname{AlN}\left(n_{2} \approx 2\right)$. 
Thus, the Brewster angle is $\theta_{B} \approx 63^{\circ}$. In this work, the ellipsometry measurements were taken at $55^{\circ}, 60^{\circ}$, and $65^{\circ}$.

\subsubsection{AIN OPTICAL MODEL}

Mahmood et al. used spectroscopic ellipsometry to determine the optical properties of reactively sputtered samples. Mahmood et al. found that when a uniform AlN film was assumed, the modeled spectra did not match the measured spectra well. However, if a three-layered model was used, the fit between the modeled and the measured data improved dramatically. Furthermore, if voids were incorporated in the middle layer (i.e. in the AlN layer), the fit was improved again. The proposed $\mathrm{Al}_{2} \mathrm{O}_{3} / \mathrm{AlN} / \mathrm{SiO}_{2}$ model for $\mathrm{AlN}$ reactively sputtered on silicon is shown in Figure 3.11.
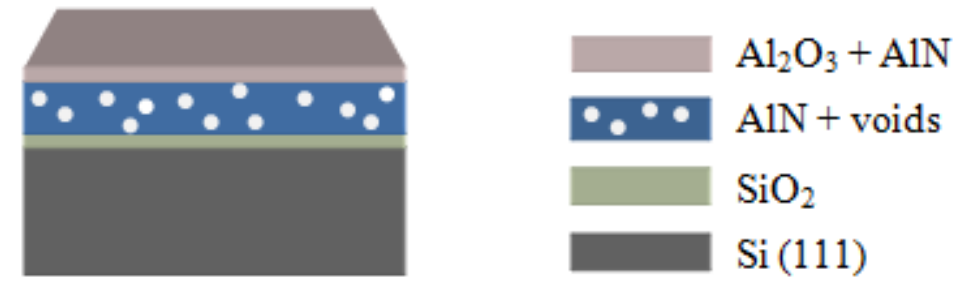

Figure 3.11. Three layer model of a reactively sputtered AlN film on silicon. After Mahmood et al. [Mahmood, 2003].

This model agrees with a similar one used by Jones et al. They modeled reactively sputtered AlN as a homogeneous thin film with interfaces represented by an effective medium approximation (EMA) layer of $50 \%$ AlN and $50 \%$ voids [Jones, 1999]. This model validates the use of several Cauchy layers and/or EMA layers that are required to fit some reactively sputtered AlN thin films.

\subsubsection{EXAMPLES FROM LITERATURE}

As discussed in Section 3.1.3.2, Mahmood et al. used spectroscopic ellipsometry to characterize reactively sputtered AIN thin films. Oscillations in the $(\psi, \Delta)$ vs. photon 
energy curves where noted when the AIN films had thicknesses on the order of $1 \mu \mathrm{m}$. It was suggested that this was due to interference effects [Mahmood, 2003].

Wang et al. performed a study on AIN thin films to determine how the ellipsometry technique compared to the more widely used XRD rocking curve method for determining the crystallinity and grain orientation. They used a similar ellipsometry setup as that used in this work. The setup consisted of a J.A. Woollam M-2000 ellipsometer capable of performing $\mathrm{VASE}^{\circledR}$ measurements. Data was collected over a spectral range of 370 to $1000 \mathrm{~nm}$ and at angles of $65^{\circ}, 70^{\circ}$, and $75^{\circ}$. The authors concluded that ellipsometry often produces comparable information as XRD on the crystallinity of AIN thin films in a fraction of the time [Wang, 2005].

Since AlN can have a refractive index between 1.8 and 2.1 depending on whether it is amorphous $(n=1.8-1.9)$, polycrystalline $(n=1.9-2.1)$, or epitaxial $(n=2.1-2.2)$ [Meng, 1994], the Brewster angle for polycrystalline AlN films should be between $62^{\circ}$ and $65^{\circ}$. In this work, a spectral range of 400 to $1000 \mathrm{~nm}$ and angles of $55^{\circ}, 60^{\circ}$, and $65^{\circ}$ (see Section 3.1.3.1) were typically used.

\subsubsection{C-V MEASUREMENTS}

Capacitance-voltage $(\mathrm{C}-\mathrm{V})$ measurements provide a useful means of determining electrical properties of Metal-Oxide-Semiconductor (MOS), Metal-InsulatorSemiconductor (MIS), and Metal-Insulator-Metal (MIM) structures, among others. Frequently, non-ideal electrical behaviors such as hysteresis are demonstrated in the C-V curves. The sense of hysteresis, hysteresis area, and shift in threshold voltage can be used to determine the density of the charges in the structure [Muller, 2003].

\subsubsection{MIS STRUCTURES}

A metal/ AlN/ silicon system can be considered to be a MIS structure due to the large bandgap of AlN (6.2 eV) [Elmazria, 2003]. The flat-band voltage, $V_{F B}$, of a MIS structure can be expressed in terms of the difference between the metal and semiconductor work functions, $\Phi_{M S}$, change in flat-band voltage due to fixed interface charge density, 
$\Delta V_{F B, Q f}$, and change in flat-band voltage due to charges in the insulator, $\Delta V_{F B, Q i}$, as given in (3.20) [Muller, 2003].

$$
V_{F B}=\Phi_{M S}+\Delta V_{F B, Q f}+\Delta V_{F B, Q i}
$$

The change in flat-band voltage due to fixed interface charge density can be expressed in terms of the total fixed charge, $Q_{f}$, and the capacitance of the insulator, $C_{i}$, as given in (3.21) [Muller, 2003].

$$
\Delta V_{F B, Q f}=-\frac{Q_{f}}{C_{i}}
$$

The change in flat-band voltage due to charges present in the insulator can be expressed in terms of the capacitance of the insulator, $C_{i}$, thickness of the insulator, $x_{i}$, and the charge distribution, $\rho(x)$, as given in (3.22) [Muller, 2003].

$$
\Delta V_{F B, Q i}=-\frac{1}{C_{i}} \int_{0}^{x_{i}} \frac{x}{x_{i}} \rho(x) d x
$$

The interface charge component can be deduced by measuring the $\mathrm{C}-\mathrm{V}$ response of the MIS structure at a high frequency (HF) and comparing this curve with the theoretical CV curve assuming that no insulator charges exist in the film. The shift in voltage between the theoretical and measured gate voltages at the insulator capacitance can be related to the interface-state density, $D_{Q f}$, as given in (3.23) where $e$ is the electron charge, $C_{i}$ is the insulator capacitance, and $\phi_{\mathrm{s}}$ is the surface potential [Muller, 2003].

$$
\left.\Delta V_{G}\right|_{C=C_{i}}=\frac{e \int_{\phi_{s 1}}^{\phi_{s 2}} D_{Q f}\left(\phi_{s}\right) d \phi_{s}}{C_{i}}
$$


Thus, C-V measurements are useful for determining the presence and origin of trapped charge states in AlN films.

\subsubsection{Silicon EleCtrical Properties}

In this work, double-side polished $p$-type (100) silicon wafers with thicknesses between $300 \mu \mathrm{m}$ and $350 \mu \mathrm{m}$ were used. The wafers were doped with boron and had a resistivity between $1 \Omega-\mathrm{cm}$ and $20 \Omega-\mathrm{cm}$. Using an iterative process, the wafers were determined to have a hole carrier concentration of approximately $10^{16}$ carriers- $\mathrm{cm}^{-3}$ and a hole mobility of approximately $330 \mathrm{~cm}^{2}-\mathrm{V}^{-1} \mathrm{~s}^{-1}$ when assuming that $\rho=10 \Omega$-cm. The intrinsic carrier concentration at room temperature was assumed to be $1.5 \times 10^{10}$ carriers$\mathrm{cm}^{-3}$. The intrinsic carrier concentration, $n_{i}$, can be related to the hole carrier concentration, $p$, and the electron carrier concentration, $n$, using the mass-action law given in (3.24).

$$
n p=n_{i}^{2}
$$

Thus, the electron carrier concentration was found to be approximately $2.25 \times 10^{4}$ carriers- $\mathrm{cm}^{-3}$. The carrier concentration of a $p$-type semiconductor can be expressed as a function of the intrinsic carrier concentration, $n_{i}$, the intrinsic Fermi level, $E_{i}$, the extrinsic Fermi level, $E_{f}$, Boltzmann's constant, $k$, and the sample temperature in degrees Kelvin, $T$, as given in (3.25) [Muller, 2003].

$$
p=n_{i} \exp \left[\frac{\left(E_{i}-E_{f}\right)}{k T}\right]
$$

Rearranging (3.25) and solving for the difference between the intrinsic and extrinsic Fermi levels gives (3.26).

$$
\left(E_{i}-E_{f}\right)=k T \ln \left(\frac{p}{n_{i}^{2}}\right)
$$


Thus, the difference, $E_{i}-E_{f}$, at room temperature $(T=300 \mathrm{~K})$ was found to be approximately $0.347 \mathrm{eV}$. The silicon parameters calculated in this section were useful in drawing the band diagrams of the MIS structures.

\subsubsection{BAND DIAGRAMS}

The band diagrams of the AIN MIS structures were simulated using the ADEPT general purpose numerical device simulator. ADEPT solves Poisson's equation and the hole and electron continuity equations in 1-d [Gray, 2007]. The input file containing the series of 'cards' used for the simulation is provided in Appendix F. The simulation results of a Pt/ AlN/ p-Si/ Pt MIS structure are plotted for an applied voltage of $0 \mathrm{~V}, 5 \mathrm{~V}$, and - 5 $\mathrm{V}$ in Figures 3.12-3.14, respectively.

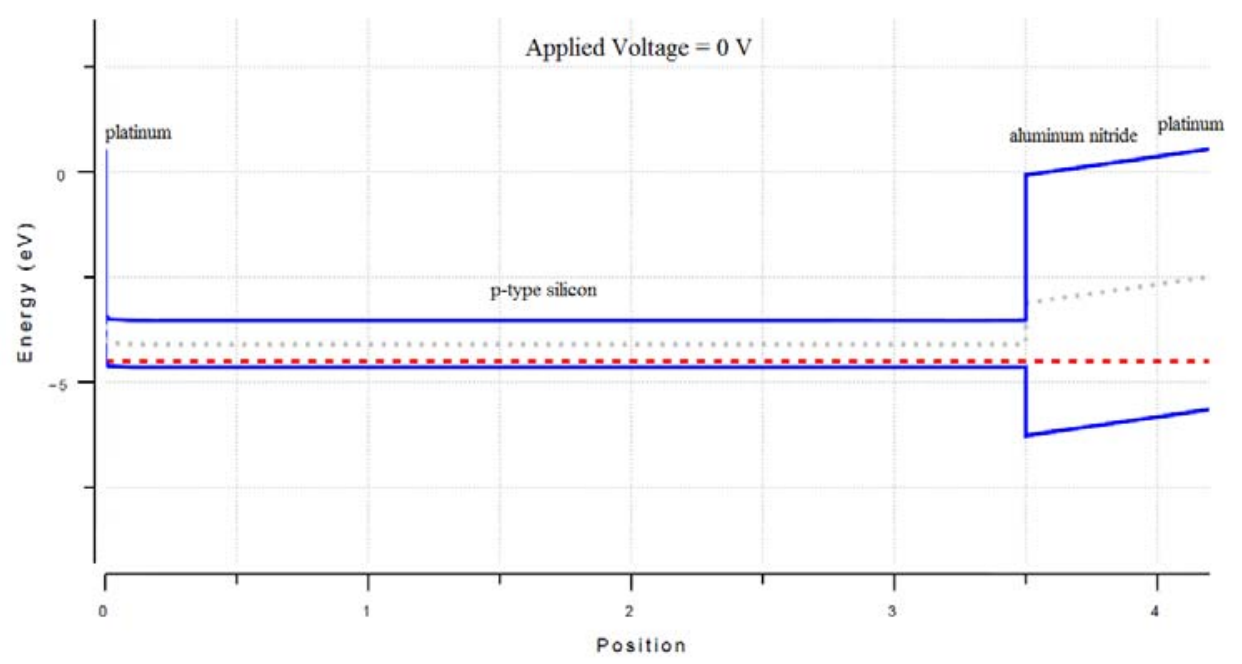

Figure 3.12. Band diagram of Pt/ AlN/p-Si/ Pt MIS structure for an applied votage of $0 \mathrm{~V}$. 


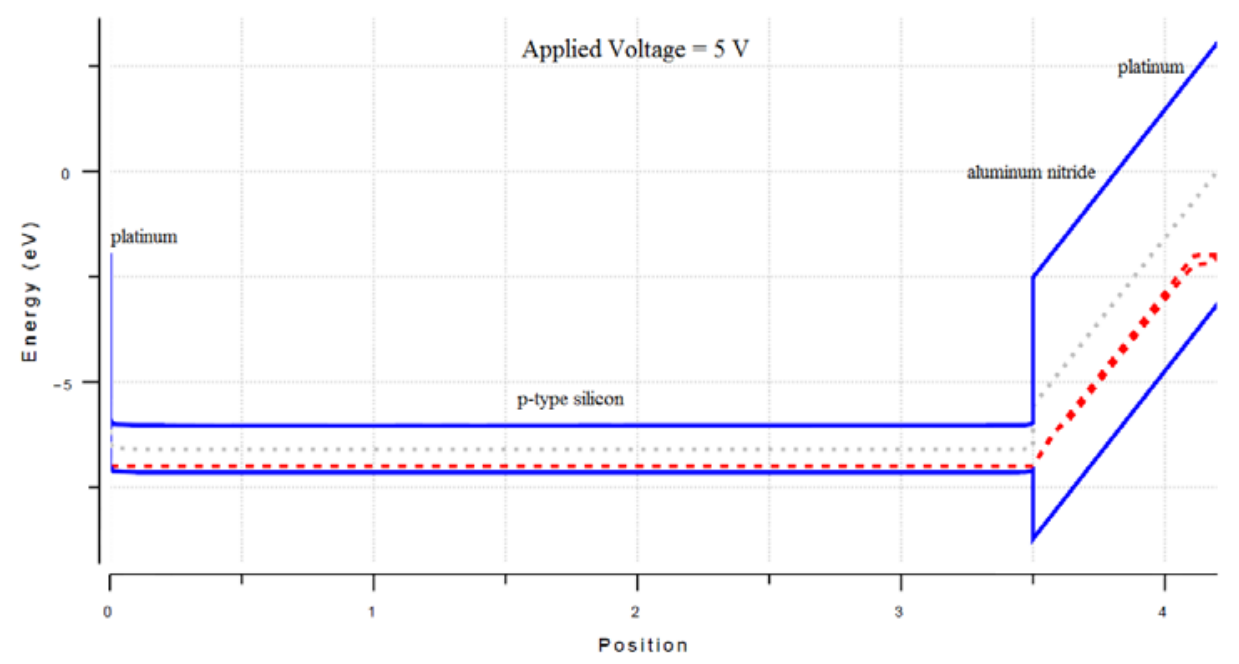

Figure 3.13. Band diagram of Pt/ AlN/p-Si/ Pt MIS structure for an applied voltage of $5 \mathrm{~V}$.

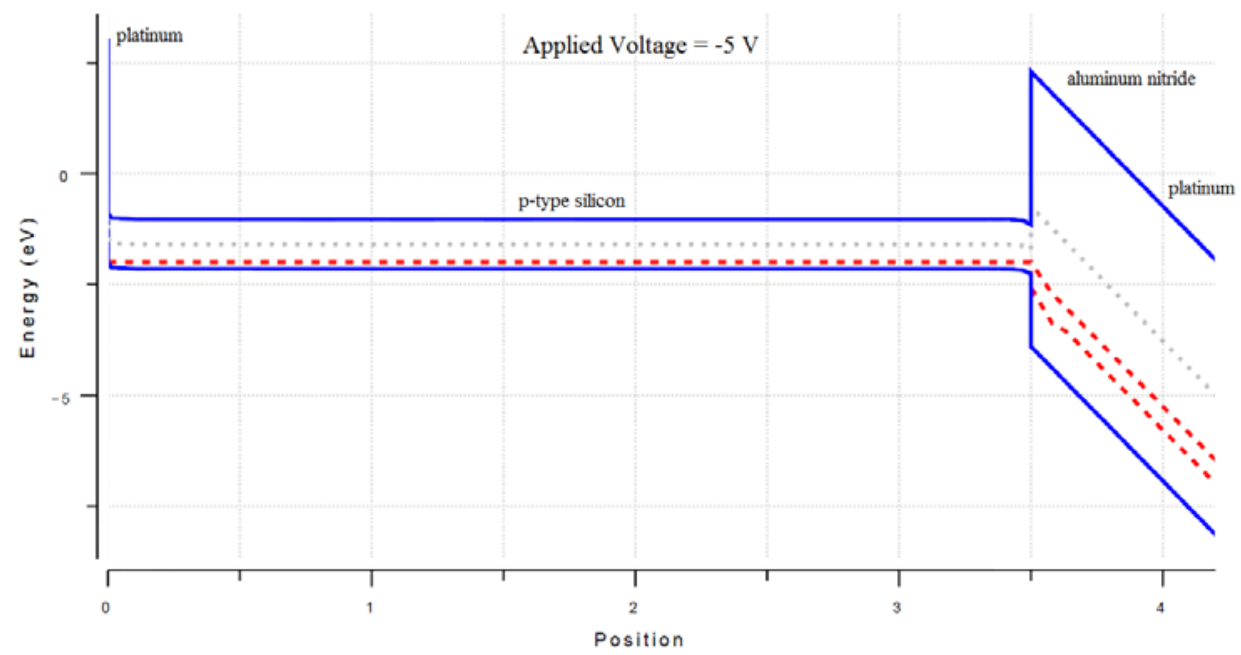

Figure 3.14. Band diagram of Pt/ AlN/p-Si/ Pt MIS structure for an applied voltage of -5 $V$.

Notice that the AIN resembles a $p$-type semiconductor even though the extrinsic carrier concentration, $N_{a}=N_{d}=0$. This is due to the much higher density of states in the valence band in comparison to that in the conduction band. However, in practice, the AlN film may be unintentionally doped $n$-type (see Section 3.2.3.5). 


\subsubsection{EXAMPLES FROM LITERATURE}

Adam et al. used C-V measurements to evaluate the electrical properties of MIS capacitors fabricated with reactively sputtered AlN films ranging from $4 \mathrm{~nm}$ to $57 \mathrm{~nm}$. Unannealed films and films annealed at $750^{\circ}$ or $800^{\circ} \mathrm{C}$ in nitrogen or oxygen environments were considered. The $\mathrm{C}-\mathrm{V}$ measurements showed hysteresis indicating that trapped charges were present in the AlN films. The films annealed in nitrogen or oxygen were found to reduce the stretching of the C-V curves [Adam, 2001].

Sharma et al. used the $\mathrm{C}-\mathrm{V}$ technique to determine the electrical properties of metal/ AlN/ silicon MIS capacitors. A $30 \mathrm{~nm}$ thick AlN film was deposited using reactive sputtering and aluminum circular contacts were deposited on top of the AlN film. A typical C-V curve of the resulting MIS structures is shown in Figure 3.15 [Sharma, 2003].



Figure 3.15. Typical C-V curve of an Al/ AlN/ $p$-Si MIS capacitor. After Sharma et al. [Sharma, 2003].

The C-V curve in Figure 3.15 has the characteristic shape for a $p$-Si substrate. The reduction in capacitance is due to the contribution of the capacitance at the AIN/Si interface (since capacitors in series add in a similar manner to resistors in parallel). 


\subsubsection{XPS}

X-ray Photoelectron Spectroscopy, or XPS, is a surface analysis technique used to determine the elemental composition, chemical bond states, and electrical structure of a sample. The physics of this technique is based upon the photoelectric effect. X-ray photons are irradiated onto the sample and are absorbed by the core electrons of the atoms comprising the sample. If the energy of the photons is large enough, the core electrons will escape from their associated atoms and will be emitted from the sample. The core electron ionization energies, $E_{i}$, can be determined using the Einstein relation given in (3.27) where $h$ is Planck's constant, $v$ is the photon frequency, and $E_{e}$ is the kinetic energy of the emitted photoelectron [McGuire, 1973].

$$
E_{i}=h v-E_{e}
$$

The ionization energy can be expressed in terms of the binding energy of the electron atomic orbital, $B E$, and the spectrometer work function, $\phi_{s}$, as expressed in (3.28) [Moulder, 1995].

$$
E_{i}=B E+\phi_{s}
$$

Since the spectrometer work function is constant, it can be neglected in (3.28) through calibration. Thus, the measured binding energy can be expressed as a function of the X-ray energy and the electron kinetic energy as given in (3.29).

$$
B E_{\text {meas }}=h v-E_{e}
$$

The two main mechanisms for X-ray photon absorption and photoelectron ejection are 1) through the direct ejection of a photoelectron and 2) through a relaxation process resulting in the emission of an Auger electron [Moulder, 1995]. These two mechanisms are shown in Figures 3.16 and 3.17. 


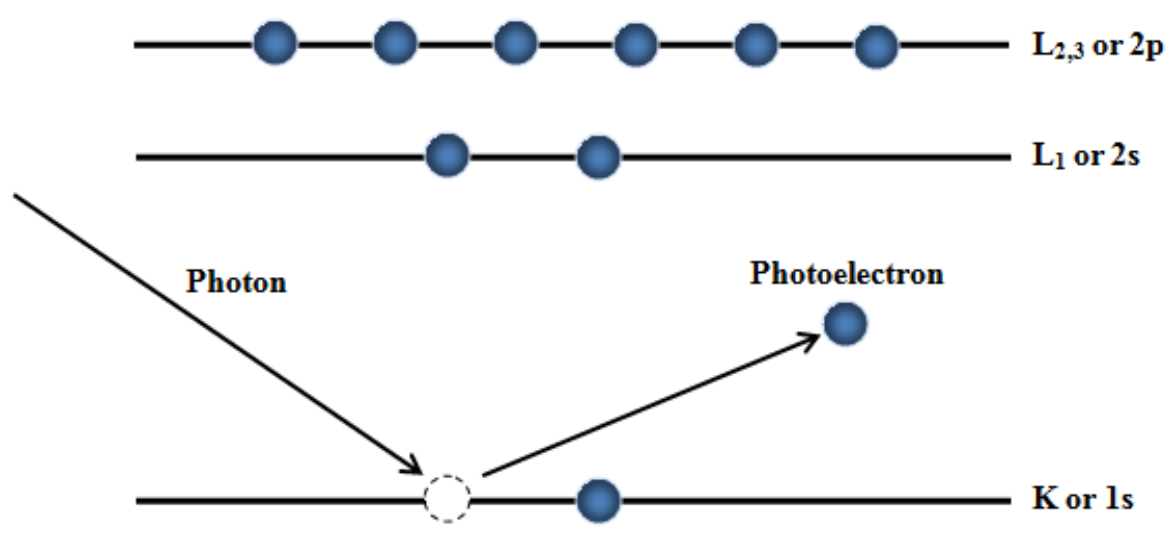

Figure 3.16. Direct photoelectron ejection mechanism. After Moulder et al. [Moulder, 1995].

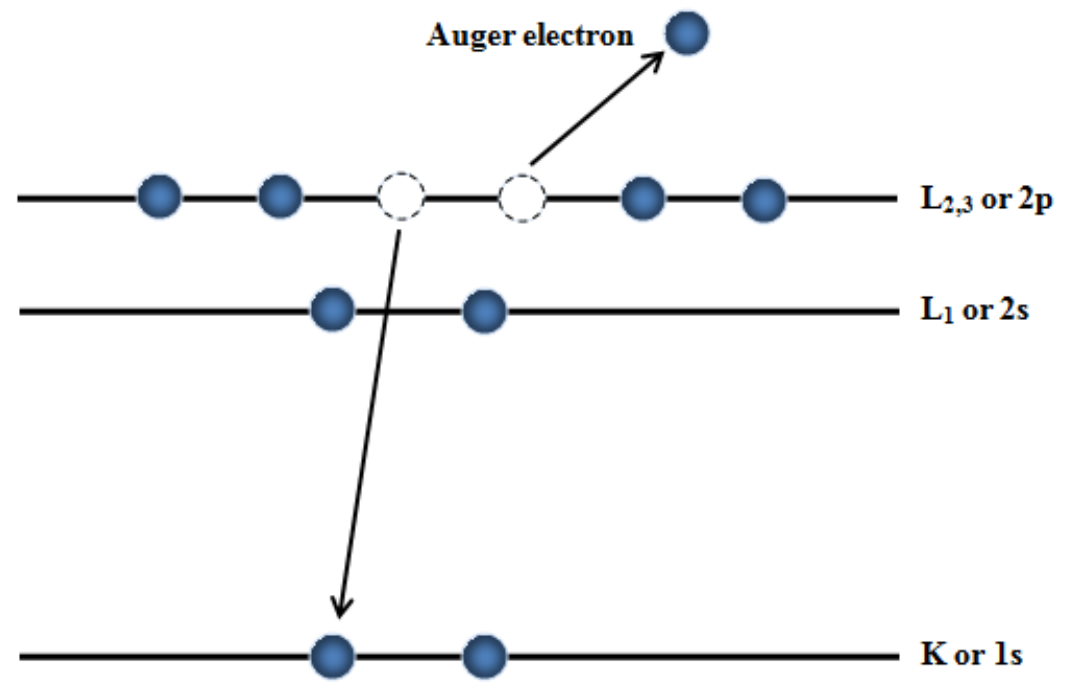

Figure 3.17. Relaxation mechanism resulting in the ejection of an Auger photoelectron. After Moulder et al. [Moulder, 1995].

\subsubsection{OPERATIONAL PARAMETERS}

The operational parameters are selected based on the sample being measured and the type of scan being performed. For survey scans, the whole range of available X-ray energies is typically scanned. So, the energy per step is set to a relatively large value $(\sim 0.5$ $\mathrm{eV})$, the time per step is set to a relatively small value $(\sim 20 \mathrm{~ms})$, the pass energy is set to a 
relatively large value $(\sim 93 \mathrm{eV})$, and the number of averaged spectra is set low $(\sim 2)$. This allows the survey scan to be acquired quickly. For detailed scans, only a portion of the range is scanned. So, the energy per step is set to a relatively small value $(\sim 0.05 \mathrm{eV})$, the time per step is set to a relatively large value $(\sim 50 \mathrm{~ms})$, the pass energy is set to a relatively small value $(\sim 23.5 \mathrm{eV})$, and the number of averaged spectra is set high $(\sim 9-16)$. This allows more accurate scans with higher Signal-to-Noise Ratios (SNRs) to be acquired around the peaks of interest.

The pass band of energies, $\Delta E$, is a function of the pass energy. As the pass energy decreases, $\Delta E$ decreases resulting in an increase in energy resolution and a decrease in sensitivity. Likewise, as the pass energy increases, $\Delta E$ increases resulting in a decrease in energy resolution and an increase in sensitivity. Thus, the pass energy can be adjusted to optimize the energy resolution/sensitivity.

\subsubsection{CHEMICAL SHIFT}

The position and the relative areas of the peaks can be used to determine the chemical status and stoichiometry of the sample. The shift in the binding energies of the core electrons, $\triangle B E$, can be compared in analogous compounds to determine the chemical status of the sample. The shift in binding energy can be related to the change in calculated charge, $\Delta q$, the change in crystal potential going from one compound to the next, $\Delta V$, the ionic radius of the ion common to both compounds, $r$, and the electron charge, $e$, as expressed in (3.30) [McGuire, 1973].

$$
\Delta B E=\left(\Delta q e^{2} / r\right)-\Delta V
$$

The ejected photoelectron is influenced by the ionic potential of ions in the lattice. Thus, the crystal potential felt by an ejected core level photoelectron can be expressed as (3.31) where $e$ is the electron charge, $q_{i}$ is the charge on an ion, and $r_{i}$ is the distance from the central ion [McGuire, 1973]. 


$$
V=\sum_{i}\left(e^{2} q_{i} / r_{i}\right)
$$

The binding energies of the core electrons of two different elements, $x$ and $y$, that form a set of analogous compounds, $1,2, \ldots, n$, can be compared using the expression in (3.32) where $\left(V_{1}-V_{2}\right)$ is the change in crystal potential [McGuire, 1973].

$$
\frac{\Delta E_{x}}{\Delta E_{y}}=\frac{\left(\Delta q_{x} e^{2} / r_{x}\right)-\left(V_{1}-V_{2}\right)_{x}}{\left(\Delta q_{y} e^{2} / r_{y}\right)-\left(V_{1}-V_{2}\right)_{y}}
$$

If the change in crystal potential is small and the charge on the ions are equal, then (3.32) can be simplified to (3.33) [McGuire, 1973]

$$
\Delta E_{x} / \Delta E_{y} \cong r_{y} / r_{x}
$$

\subsubsection{Elemental COMPOSITION}

For a sample that is homogeneous over the sampling volume, the number of photoelectrons generated per second for a particular peak can be expressed as (3.34) where $n$ is the density of atoms of the particular element per $\mathrm{cm}^{3}, f$ is the x-ray flux in photons $/ \mathrm{cm}^{2}$-sec, $\sigma$ is the photoelectric cross-section in $\mathrm{cm}^{2}, \theta$ is the angular efficiency factor, $y$ is the photoelectric efficiency, $\lambda$ is the photoelectron mean free path, $A$ is the sample area in which photoelectrons are detected, and $T$ is the detection efficiency [Moulder, 1995].

$$
I=n f \sigma \theta y \lambda A T
$$

Equation (3.34) can be rearranged to solve for $n$ as given in (3.35) where $S$ is typically defined as the Atomic Sensitivity Factor (ASF) of a particular element [Moulder, 1995]. 


$$
n=I / f \sigma \theta y \lambda A T=I / S
$$

The ratio of the atomic densities of two elements can be expressed as (3.36) [Moulder, 1995].

$$
\frac{n_{1}}{n_{2}}=\frac{I_{1} / S_{1}}{I_{2} / S_{2}}
$$

Additionally, relative values of the sensitivity factors can be tabulated for a given spectrometer since the ratios $\sigma_{1} / \sigma_{2}$ and $\lambda_{1} / \lambda_{2}$ remain nearly constant. The atomic sensitivity factors of some elements encountered in this work are provided in Table 3.1 [Moulder, 1995].

Table 3.1. Atomic sensitivity factors of spectral lines of relevance to this work. After Moulder et al. [Moulder, 1995].

\begin{tabular}{l|l|l}
\hline Element & Line & ASF \\
\hline $\mathrm{Al}$ & $2 \mathrm{p}$ & 0.234 \\
\hline $\mathrm{C}$ & $1 \mathrm{~s}$ & 0.296 \\
\hline $\mathrm{Er}$ & $4 \mathrm{~d}$ & 2.463 \\
\hline $\mathrm{F}$ & $1 \mathrm{~s}$ & 1.000 \\
\hline $\mathrm{N}$ & $1 \mathrm{~s}$ & 0.477 \\
\hline $\mathrm{O}$ & $1 \mathrm{~s}$ & 0.711 \\
\hline $\mathrm{Si}$ & $2 \mathrm{p}$ & 0.339 \\
\hline
\end{tabular}

The atomic concentration of an element in the sample can be determined using the expression given in (3.37) [Moulder, 1995].

$$
C_{x}=\frac{n_{x}}{\sum n_{i}}=\frac{I_{x} / S_{x}}{\sum I_{i} / S_{i}}
$$


The SNR, $I_{S} / I_{N}$, increases as the number of averaged spectra, $n$, is increased as given by (3.38) and (3.39).

$$
\begin{aligned}
& C_{1}=\frac{I_{S 1}}{I_{N 1}}, C_{n}=\frac{I_{S N}}{I_{N 1}} \\
& \frac{C_{n}}{C_{1}} \alpha \sqrt{n}
\end{aligned}
$$

However, there is a trade-off between increased SNR and increased measurement time. Therefore, the number of averaged spectra is typically set between 2 and 5 when performing a survey scan and between 10 and 20 when performing a detailed scan.

\subsubsection{ARXPS}

XPS is a surface technique capable of providing information from roughly three times the Inelastic Mean Free Path (IMFP), or $3 \lambda$, into the sample. The IMFP is defined as the average distance in which an electron with kinetic energy travels between successive collisions. For most materials, the IMFP is between $1 \mathrm{~nm}$ and $3 \mathrm{~nm}$. Thus, XPS can provide information about the top $3 \mathrm{~nm}$ to $10 \mathrm{~nm}$ of the sample. However, the detection

depth can be varied using a technique called Angle-Resolved (AR) XPS. ARXPS allows the degree of surface sensitivity to be adjusted by varying the emission angle $\theta$. This can be expressed as (3.40) where $x$ is the sampling depth, $\lambda$ is the IMFP, $d \approx 3 \lambda$ is the escape depth, and $\theta$ is the emission angle.

$$
x=3 \lambda \cos \theta
$$

Angle resolved XPS is useful for performing non-destructive depth profiling of the top $3 \lambda$ into the sample. 


\subsubsection{Destructive DePth Profiling}

Destructive depth profiling is a technique that incorporates sputtering step(s) to remove material from the surface of the sample. This enables XPS to provide information about the material beneath the top $3 \mathrm{~nm}$ to $10 \mathrm{~nm}$. Typically, an ion argon gun is used to bombard the sample surface and remove material. Sometimes this is called "sputtering" the sample since the process resembles the sputtering deposition technique discussed in Section 2.2.1. The energy of the ions bombarding the surface of the sample can be adjusted allowing the sputter rate to be controlled. Additionally, the sputtered area should be much greater than the X-ray spot size so that the side profiles of the sputtered region do not skew the results.

\subsubsection{EXAMPLES FROM LITERATURE}

Engelmark et al. used XPS to monitor the impurity levels in reactively sputtered aluminum nitride thin films. They found that one of the most common impurities in the films was oxygen (see Section 2.3.2). Figure 3.18 shows a typical XPS survey scan spectra for reactively sputtered polycrystalline AIN. The only impurity present in the film is that of oxygen. The atomic concentration of oxygen was found to be $0.5 \%$ [Engelmark, 2000].

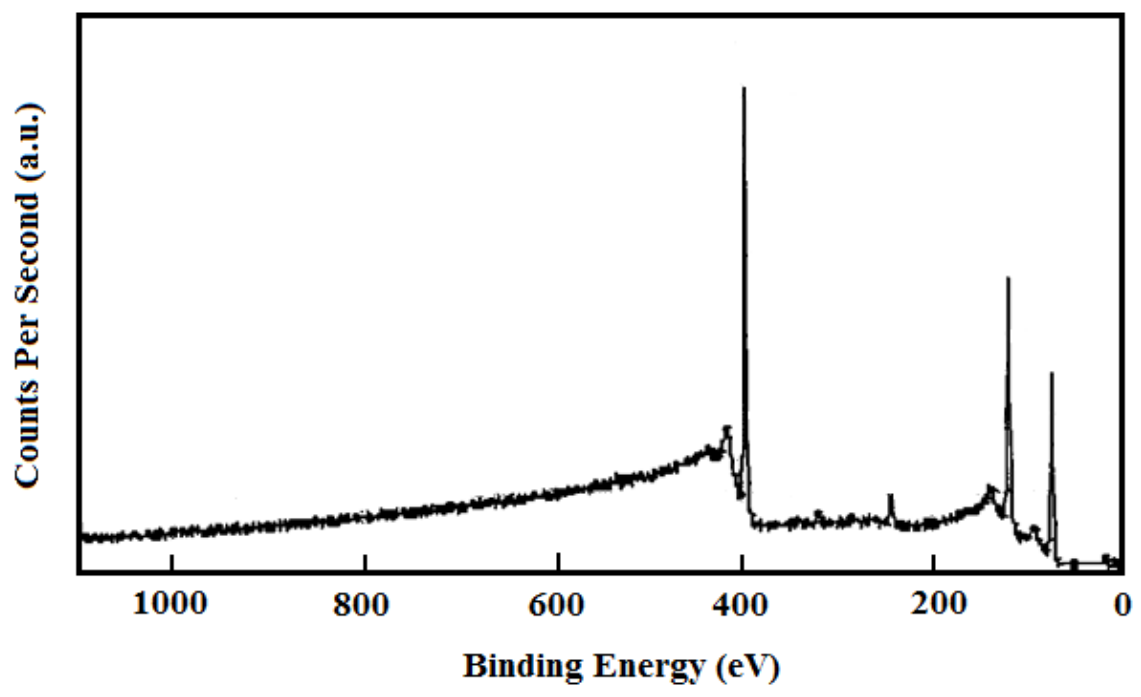

Figure 3.18. XPS survey scan spectra of reactively sputtered AlN. After Engelmark et al. [Engelmark, 2000]. 
Sharma et al. also used XPS to characterize reactively sputtered aluminum nitride thin films. Sharma et al. found that the films had atomic concentrations of oxygen of upto $23.09 \%$ which is much higher than that found by Engelmark et al. Additionally, they found that the ratio of aluminum to nitrogen in the films was approximately 1:1. The survey scan spectra and the detail scan spectra of the Al2p are shown in Figures 3.19 and 3.20 , respectively [Sharma, 2003].

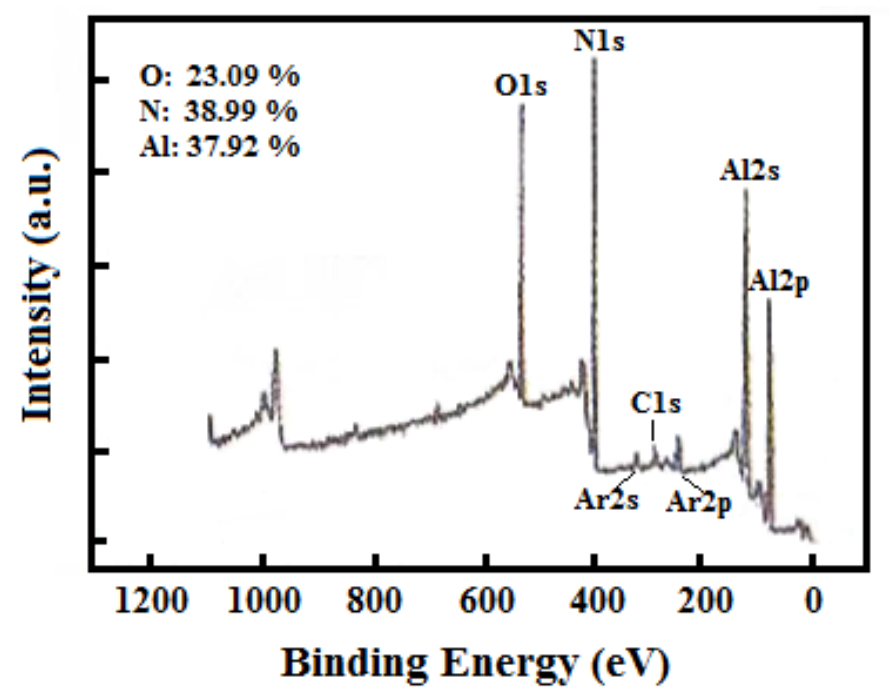

Figure 3.19. XPS survey scan spectra of reactively sputtered AlN. After Sharma et al. [Sharma, 2003]. 


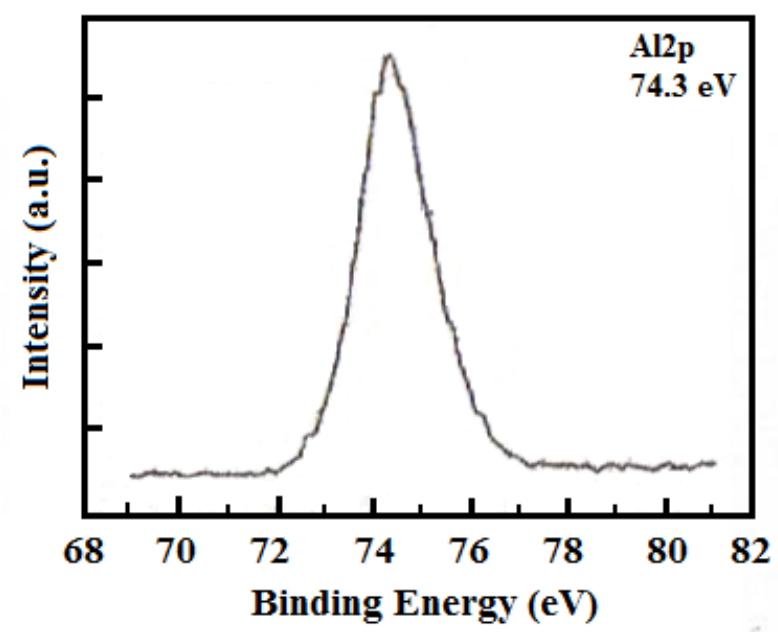

Figure 3.20. XPS detailed scan spectra the Al2p peak of reactively sputtered AlN. After Sharma et al. [Sharma, 2003].

Watanabe et al. used XPS to determine how ion beam assisted deposited aluminum nitride films oxidized when annealed at $900^{\circ} \mathrm{C}$ (see Section 2.3.4). They annealed AlN films with columnar and granular microstructures for 5 minutes at $900^{\circ} \mathrm{C}$ in an oxygen environment. The XPS depth profiles for the two types of films are shown in Figures 3.21 and 3.22, respectively [Watanabe, 2000].



Figure 3.21. XPS depth profile of a columnar AlN film after annealing. After Watanabe et al. [Watanabe, 2000]. 


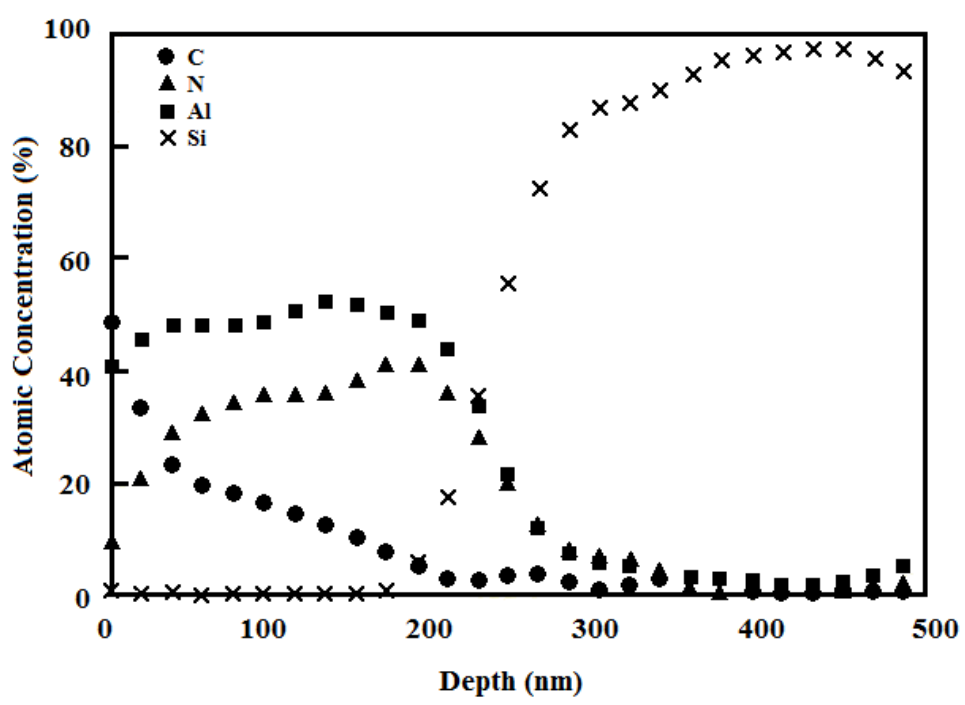

Figure 3.22. XPS depth profile of a granular AlN film after annealing. After Watanabe et al. [Watanabe, 2000].

As shown in Figures 3.21 and 3.22, the oxidation of AlN depends on its microstructure. Films with lower crystallinity (i.e. the granular film) were shown to oxidize more readily than films with higher crystallinity (i.e. the columnar film). Watanabe et al. suggested that this was due to lower oxygen diffusivity in columnar films compared to granular films [Watanabe, 2000].

Bernhardt et al. used XPS to determine the BE of Rare Earth (RE) ions. They used an aluminum X-ray source $\left(E_{p h}=1486.6 \mathrm{eV}\right)$, so the binding energies of Er $3 d$ through Lu $3 d$ electrons could not be determined $\left(\mathrm{BE}>E_{p h}\right)$. However, the authors were able to obtain the binding energies of the $4 d$ electrons of all of the trivalent lanthanoids. The results are provided in Table 3.2 [Bernhardt, 2002]. 
Table 3.2. Binding energies of rare earth ions. After Bernhardt et al. [Bernhardt, 2002].

\begin{tabular}{l|c|c|c|c}
\hline \multicolumn{1}{c|}{ Rare Earth } & BE $4 \boldsymbol{d}(\mathbf{e V})$ & $\mathbf{B E ~} 3 \boldsymbol{d}_{\mathbf{5} / \mathbf{2}}(\mathbf{e V})$ & $\mathbf{B E} \mathbf{3} \boldsymbol{d}_{\mathbf{3} / \mathbf{2}}(\mathbf{e V})$ & $\boldsymbol{\Delta E}_{\mathbf{3} \boldsymbol{d}}(\mathbf{e V})$ \\
\hline Lanthanum (La) & 104.2 & 841.3 & 858.0 & 16.7 \\
\hline Cerium (Ce) & 111.3 & 884.6 & 903.1 & 18.5 \\
\hline Praseodymium (Pr) & 116.4 & 933.6 & 953.9 & 20.3 \\
\hline Neodymium (Nd) & 122.1 & 982.6 & 1004.7 & 22.1 \\
\hline Samarium (Sm) & 133.2 & 1083.1 & 1110.5 & 27.4 \\
\hline Europium (Eu) & 139.1 & 1134.8 & 1164.1 & 29.3 \\
\hline Gadolinium (Gd) & 141.9 & 1187.1 & 1219.1 & 32.0 \\
\hline Terbium (Tb) & 150.6 & 1241.9 & 1276.9 & 35.0 \\
\hline Dysprosium (Dy) & 156.5 & 1296.4 & 1335.4 & 39.0 \\
\hline Holmium (Ho) & 162.0 & 1352.5 & 1394.2 & 41.7 \\
\hline Erbium (Er) & 169.0 & & & \\
\hline Thulium (Tm) & 176.4 & & & \\
\hline Ytterbium (Yb) & 185.4 & & & \\
\hline Lutetium (Lu) & $197.1\left(4 d_{5 / 2}\right)$ & & & \\
\hline & $207.1\left(4 d_{3 / 2}\right)$ & & & \\
\hline
\end{tabular}

Additionally, Bernhardt et al. plotted the binding energies of the $3 d$ and $4 d$ electrons as a function of the rare earth ions as shown in Figure 3.23 [Bernhardt, 2002]. 


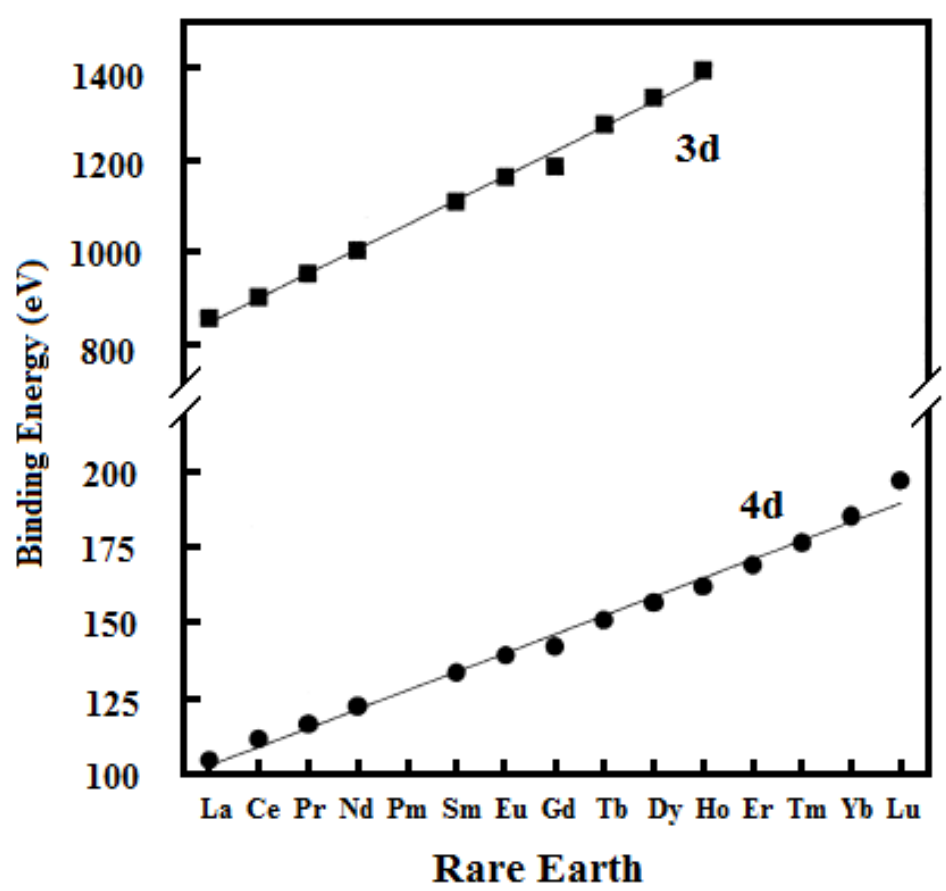

Figure 3.23. Binding energies of the $3 d$ and $4 d$ electrons of rare earth ions. After Bernhardt et al. [Bernhardt, 2002].

In this work, XPS was used to determine the films composition as a function of thickness and to look for trace elements of erbium $(\mathrm{BE}=169 \mathrm{eV})$ in Er-doped AlN thin films.

\subsection{CASE STUdies}

The characterization techniques presented in Section 3.1 were used to determine the properties of ultrananocrystalline diamond films, undoped AlN films, and Er-doped AlN films. Additionally, the electrical properties of AIN MIS structures were investigated. These case studies are presented in this section.

\subsubsection{UNCD}

Diamond films possess the highest acoustic wave velocity of all materials [Benetti, 2005]. UltraNanoCrystalline Diamond (UNCD) films are high quality epitaxial films grown using a chemical vapor process that have been found to have similar qualities as their crystalline diamond counterparts. UNCD films have been used as the substrate for 
Zinc Oxide $(\mathrm{ZnO})$ and Aluminum Nitride (AlN) Surface Acoustic Wave (SAW) devices [Nakahata, 1995; Benetti, 2005; Fansler, 2008]. The use of UNCD films has shown to increase functional capabilities of the SAW devices such as the acoustic wave velocity and electrical coupling coefficient [Nakahata, 1995; Fansler, 2008]. This allows the SAW devices to operate at higher frequencies than normally possible for a given InterDigitated Transducer (IDT) line and space [Benetti, 2005].

In this section, UNCD Diamond films on Silicon (DoSi) wafer samples were provided by Advanced Diamond Technologies, Inc. (www.thindiamond.com). The films were estimated to have a nominal thickness of $1 \mu \mathrm{m}$. In this work, the films were characterized using VASE ${ }^{\circledR}$ and AFM. The VASE ${ }^{\circledR}$ technique was used to determine the thickness and optical properties of the films and the AFM technique was used to determine the surface roughness and topography of the UNCD films.

\subsubsection{1 $\operatorname{VASE}^{\circledR}$}

Ellipsometry measurements were performed on UNCD films over the spectral range of $245 \mathrm{~nm}$ to $1000 \mathrm{~nm}$ and at angles of $55^{\circ}, 60^{\circ}$, and $65^{\circ}$ using a J. A. Woollam M$2000 U^{\mathrm{TM}}$ spectroscopic ellipsometer. The data was fit with a two coupled Cauchy layers and an overlying surface roughness layer using the WVASE32 Version 3.464 software provided with the equipment. The optical model and the corresponding thicknesses determined by fitting the experimental data with the model data over the spectral range of $400 \mathrm{~nm}$ to $1000 \mathrm{~nm}$ are given in Table 3.3.

Table 3.3. Optical model for UNCD over the spectral range of $400 \mathrm{~nm}$ to $1000 \mathrm{~nm}$.

\begin{tabular}{l|l|l}
\hline \multicolumn{1}{c|}{ Layer } & \multicolumn{1}{|c}{ Name } & \multicolumn{1}{c}{ Thickness } \\
\hline 3 & Srough & $9.318 \mathrm{~nm}$ \\
\hline 2 & Cauchy2 & $47.563 \mathrm{~nm}$ \\
\hline 1 & Cauchy & $804.275 \mathrm{~nm}$ \\
\hline 0 & 3 inch si substrate & $1 \mathrm{~mm}$ \\
\hline
\end{tabular}

The MSE for this fit was 79.52 and the ratio of MSE to thickness was determined to be less than $10 \%$. However, the model did not fit the experimental data perfectly. Thus, 
the experimental data was fit again over a reduced spectral range in order to decrease the MSE.

The optical model and the corresponding thicknesses for the spectral range of $700 \mathrm{~nm}$ to $1000 \mathrm{~nm}$ is shown in Table 3.4.

Table 3.4. Ellipsometry model for UNCD over the spectral range of $700 \mathrm{~nm}$ to $1000 \mathrm{~nm}$.

\begin{tabular}{l|l|l}
\hline \multicolumn{1}{c|}{ Layer } & \multicolumn{1}{|c}{ Name } & \multicolumn{1}{c}{ Thickness } \\
\hline 3 & Srough & $0.000 \mathrm{~nm}$ \\
\hline 2 & Cauchy2 & $61.717 \mathrm{~nm}$ \\
\hline 1 & Cauchy & $800.283 \mathrm{~nm}$ \\
\hline 0 & 3 inch si substrate & $1 \mathrm{~mm}$ \\
\hline
\end{tabular}

The MSE for this fit was 30.19 and the ratio of MSE to thickness was determined to be less than $5 \%$. However, the fit indicated that the film was perfectly smooth (contradicting the AFM results presented in Section 3.2.1.2).

\subsubsection{AFM}

The surface roughness and grain height of the UNCD thin films were determined using tapping-mode Atomic Force Microscopy (AFM). A Veeco multimode scanning probe was used to perform the measurements. Three scans with different scan sizes were performed. Figure 3.24 shows the topography of the UNCD film in a $500 \mathrm{~nm}$ x $500 \mathrm{~nm}$ area. The parameters used for this scan were scan size $=500 \mathrm{~nm}$, scan rate $=0.2501 \mathrm{~Hz}$, number of samples $=512$, and data scale $=50 \mathrm{~nm}$. 


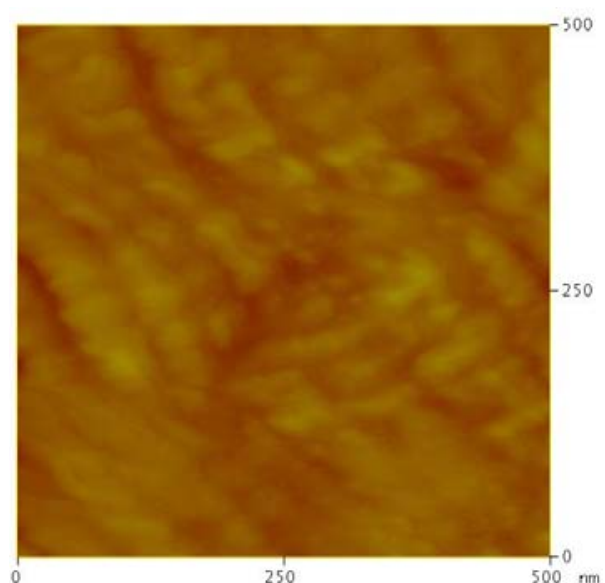

(a)



(b)

Figure 3.24. $500 \mathrm{~nm} \times 500 \mathrm{~nm}$ scan of an UNCD film. (a) 2-d topography image and (b) 3d topography image.

Figure 3.25 shows the topography of the same UNCD film in a $2 \mu \mathrm{m} \times 2 \mu \mathrm{m}$ area. The parameters used for this scan were: scan size $=2 \mu \mathrm{m}$, scan rate $=0.2501 \mathrm{~Hz}$, number of samples $=512$, and data scale $=50 \mathrm{~nm}$.

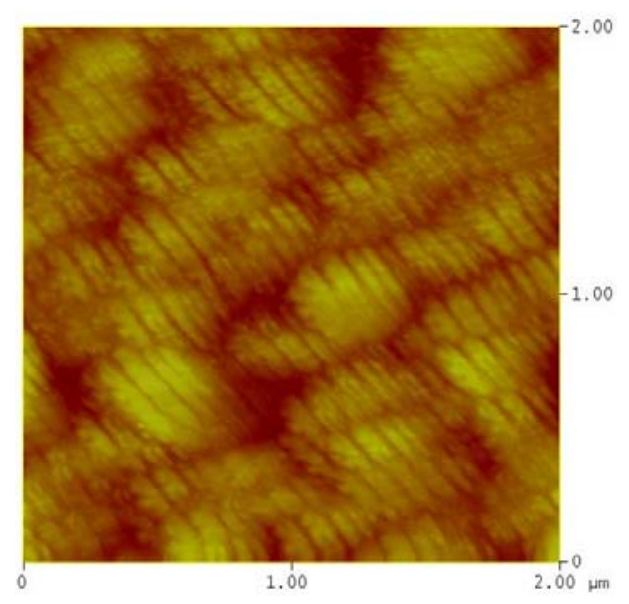

(a)

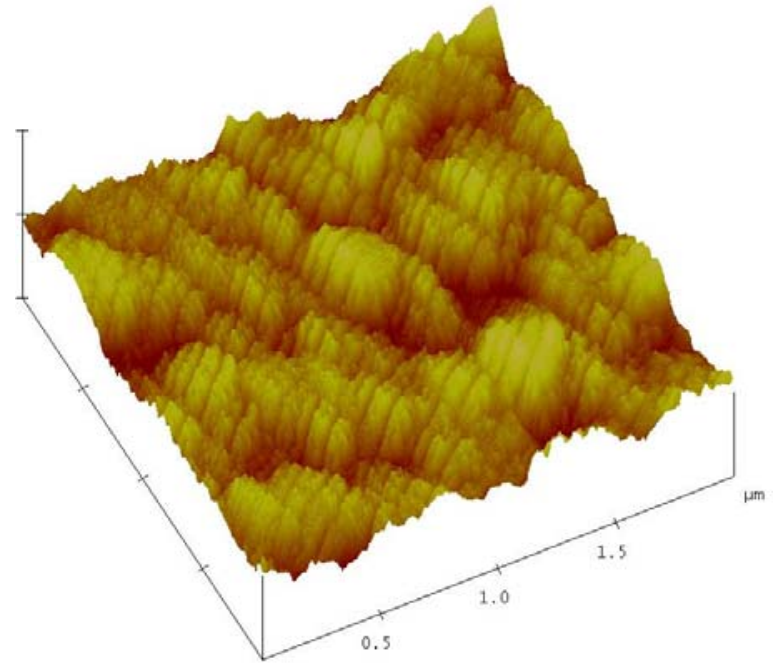

(b)

Figure 3.25. $2 \mu \mathrm{m} \times 2 \mu \mathrm{m}$ scan of an UNCD film. (a) 2-d topography image and (b) 3-d topography image. 
Figure 3.26 shows the topography of the same UNCD film in a $5 \mu \mathrm{m} \times 5 \mu \mathrm{m}$ area. The parameters used for this scan were scan size $=2 \mu \mathrm{m}$, scan rate $=0.5003 \mathrm{~Hz}$, number of samples $=512$, and data scale $=50 \mathrm{~nm}$.

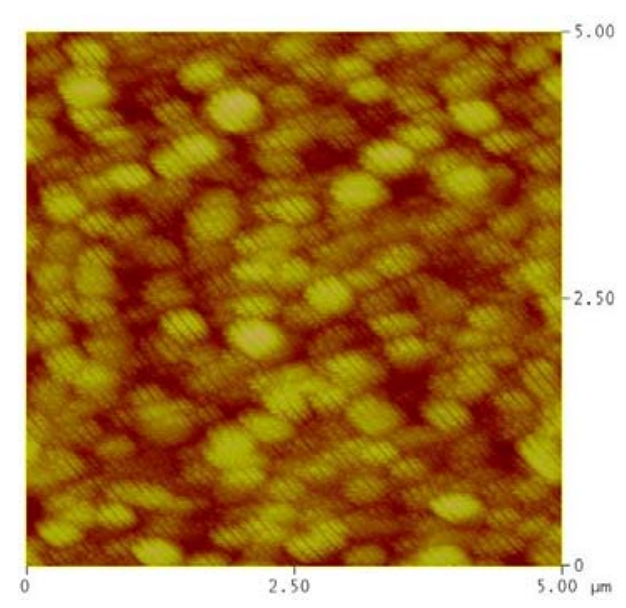

(a)



(b)

Figure 3.26. $5 \mu \mathrm{m} \times 5$ um scan of an UNCD film. (a) 2-d topography image and (b) 3-d topography image.

Using the Nanoscope software provided with the AFM system, the rms roughness for $500 \mathrm{~nm}, 2 \mu \mathrm{m}$, and $5 \mu \mathrm{m}$ scans were found to be $1.826 \mathrm{~nm}, 4.797 \mathrm{~nm}$, and $6.587 \mathrm{~nm}$. It is interesting to note that the calculated surface roughness was found to increase with the scan area. It is thought that this variation is due to the large grain size present in the films.

\subsubsection{SUMMARY}

By combining the information, it can be determined that the UNCD layer was approximately $850 \mathrm{~nm}$ thick and had a surface roughness of less than $10 \mathrm{~nm}$. This thickness is $150 \mathrm{~nm}$ thinner than indicated by the vendor. Ideally, the thickness of the $\mathrm{UNCD}$ should be over $3 \lambda$ and the surface roughness should be $\sim 0 \mathrm{~nm}$ for use in SAW devices [Fansler, 2008]. As discussed in Section 5.2.1, the acoustic wavelength corresponding to the IDTs fabricated in this work is $8 \mu \mathrm{m}$. Thus, an UNCD thickness of 24 $\mu \mathrm{m}$ is desired. A perfectly smooth surface is ideal because it reduces the propagation loss 
in the SAW device [Fansler, 2008]. If the roughness of the UNCD film increases as a function of thickness, the losses in the SAW devices due to the roughness of the UNCD film may diminish the benefits gained from using the UNCD coated substrates. Also, note the similarity of Figures 3.24-3.25 to Figure 3.4(a).

\subsubsection{AIN AND ER-DOPED AIN}

Aluminum nitride is a promising material for SAW devices due to its high acoustic velocity and favorable electrical, mechanical, and chemical properties [Benetti, 2007]. However, AlN based SAW devices experience a significant insertion loss due to AlN's relatively low piezoelectric constants. The most direct method of reducing the insertion loss is to improve the piezoelectric response of the AlN layer. It has recently been shown that incorporating rare earth metals such as Erbium (Er) into the AlN layer leads to a higher piezoelectric response [Kabulski, 2009].

Three AlN and three Er-doped AlN films were characterized using VASE ${ }^{\circledR}$, SEM, AFM, and XPS. Full 3" silicon wafers were used in this study. Since many of the characterization techniques discussed require small samples, pieces of the AlN coated wafer were cut from the full 3" wafer. The origin of the pieces taken from the 3" wafer are shown schematically in Figure 3.27.



Figure 3.27. Schematic of an AlN thin film on a 3" silicon wafer. 


\subsubsection{DEPOSITION PARAMETERS}

AlN films and Er-doped AlN films with nominal thicknesses of 0.1, 0.3, and 0.5 $\mu \mathrm{m}$ were reactively sputtered in separate runs using a CVC 610 DC magnetron sputter deposition system. The substrates used for all depositions were 3", (100) p-type, doubleside polished, 1-20 $\Omega$-cm resistivity silicon wafers purchased from Wafer World, Inc. (www.waferworld.com). Prior to loading the wafers in the sputtering chamber, the wafers were cleaned in a 5 minute acetone bath, a 5 minute methanol bath, and a 5 minute dip in 10:1 deionized (DI) water to J.T. Baker $49 \%$ hydrofluoric acid (HF). The wafers were rinsed in a cascading bath of DI water for 10 minutes, blown dry with nitrogen gas, and baked in a gravity oven for 10 minutes at $110^{\circ} \mathrm{C}$.

A $50 \mathrm{~mm}$-diameter, $99.999 \%$ pure $\mathrm{Al}$ target and a $50 \mathrm{~mm}$-diameter, $99.9 \%$ $\mathrm{Al}_{0.97} \mathrm{Er}_{0.03}$ target were used to deposit undoped $\mathrm{AlN}$ films and Er-doped AlN films, respectively. Prior to deposition, the sputtering chamber was pumped to a base pressure below $5 \times 10^{-6}$ Torr using a CTI-8 cryopump. Since the $\mathrm{Al}$ and the $\mathrm{Al}_{0.97} \mathrm{Er}_{0.03}$ targets were found to oxidize when stored in an ambient environment, a two step pre-sputter was performed to remove the surface oxidation/contamination and coat the target with a thin layer of AlN. The pre-sputter was immediately followed by film deposition. All presputters and depositions were performed with a magnetron power of $100 \mathrm{~W}$ and a working pressure of $45 \mathrm{mTorr}$. The pre-sputtering and sputtering parameters are provided in Table 3.5 .

Table 3.5. Pre-sputtering and sputtering parameters.

\begin{tabular}{l|c|c|c|c}
\hline & Shutter status & Ar flow rate & $\mathbf{N}_{\mathbf{2}}$ flow rate & Sputtering time \\
\hline $\begin{array}{l}\text { Pre-sputtering } \\
\text { Step 1 }\end{array}$ & Closed & $30 \mathrm{sccm}$ & $0 \mathrm{sccm}$ & $10 \mathrm{~min}$. \\
\hline $\begin{array}{l}\text { Pre-sputtering } \\
\text { Step 2 }\end{array}$ & Closed & $3 \mathrm{sccm}$ & $27 \mathrm{sccm}$ & $5 \mathrm{~min}$. \\
\hline $\begin{array}{l}\text { AlN \& Er-doped } \\
\text { AlN depositions }\end{array}$ & Open & $3 \mathrm{sccm}$ & $27 \mathrm{sccm}$ & Varied \\
\hline
\end{tabular}




\subsubsection{ELLIPSOMETRY}

Ellipsometry measurements were performed on the undoped and Er-doped AIN films over the spectral range of $245 \mathrm{~nm}$ to $1000 \mathrm{~nm}$ and at angles of $55^{\circ}, 60^{\circ}$, and $65^{\circ}$ using a J. A. Woollam M-2000U ${ }^{\mathrm{TM}}$ spectroscopic ellipsometer. The data was fit using a single Cauchy layer and an overlying surface roughness layer using the WVASE32 Version 3.464 software provided with the equipment. The optical parameters and thickness were determined by fitting the experimental data to the model data over the spectral range of 400 $\mathrm{nm}$ to $1000 \mathrm{~nm}$. The information derived using $\mathrm{VASE}^{\circledR}$ are provided in Table 3.6.

Table 3.6. Information derived using $V A S E^{\circledR}$.

\begin{tabular}{|c|c|c|c|c|c|c|}
\hline & MSE & $\begin{array}{c}\text { Thick } 1 \\
\text { (nm) }\end{array}$ & $\begin{array}{c}\text { Deposition } \\
\text { Rate } \\
\text { (nm/min) }\end{array}$ & $\begin{array}{c}\text { MSE/ } \\
\text { Thick } 1 \\
\left(\mathrm{~nm}^{-1}\right)\end{array}$ & $\begin{array}{c}\text { n@ } \\
588.947 \\
\text { nm }\end{array}$ & Fit \\
\hline $\begin{array}{l}0.1 \mu \mathrm{m} \text { Un-AlN } \\
(17.8 \mathrm{~min})\end{array}$ & 5.796 & 98.292 & 5.522 & 0.059 & 1.9677 & Exc \\
\hline $\begin{array}{l}0.3 \mu \mathrm{m} \text { Un-AlN } \\
(53.2 \mathrm{~min})\end{array}$ & 8.638 & 282.755 & 5.315 & 0.031 & 2.0045 & Exc \\
\hline $\begin{array}{l}0.5 \mu \mathrm{m} \text { Un-AlN } \\
(88.7 \mathrm{~min})\end{array}$ & 14.46 & 464.116 & 5.232 & 0.031 & 2.0471 & Exc \\
\hline $\begin{array}{l}0.1 \mu \mathrm{m} \text { Er-AlN } \\
(9.6 \mathrm{~min})\end{array}$ & 6.601 & 77.623 & 8.086 & 0.085 & 1.9947 & Exc \\
\hline $\begin{array}{l}0.3 \mu \mathrm{m} \text { Er-AlN } \\
(28.9 \mathrm{~min})\end{array}$ & 43.18 & 365.147 & 12.635 & 0.118 & 2.1031 & Poor \\
\hline $\begin{array}{l}0.5 \mu \mathrm{m} \text { Er-AlN } \\
(48.1 \mathrm{~min})\end{array}$ & 72.49 & 527.885 & 10.975 & 0.137 & 1.9342 & Poor \\
\hline
\end{tabular}

The optical properties determined from the $\mathrm{VASE}^{\circledR}$ measurement were feed into the VASE Manager software and surface maps of thickness verses position were generated at a single angle at 349 points over the films. The surface maps of the undoped AlN films are shown in Figures 3.28(a)-3.30(a) and the surface maps of the Er-doped films are shown in Figures 3.28(b)-3.30(b). 


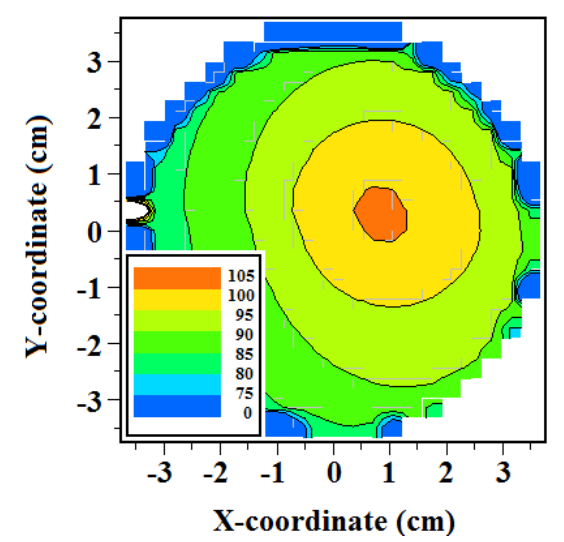

(a)

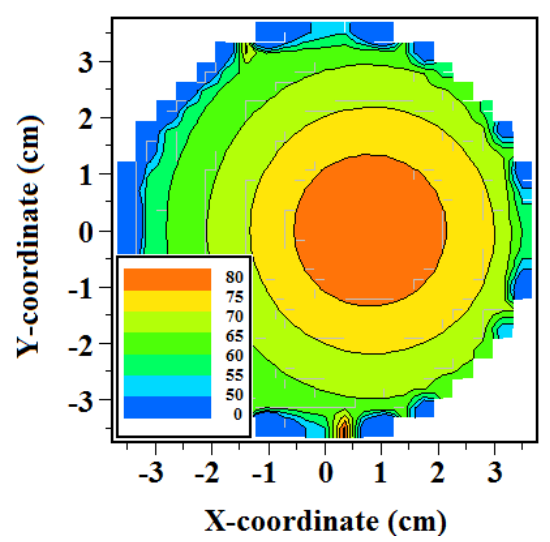

(b)

Figure 3.28. Surface map of AlN films with a nominal thickness of $0.1 \mu \mathrm{m}$. (a) undoped and (b) Er-doped.

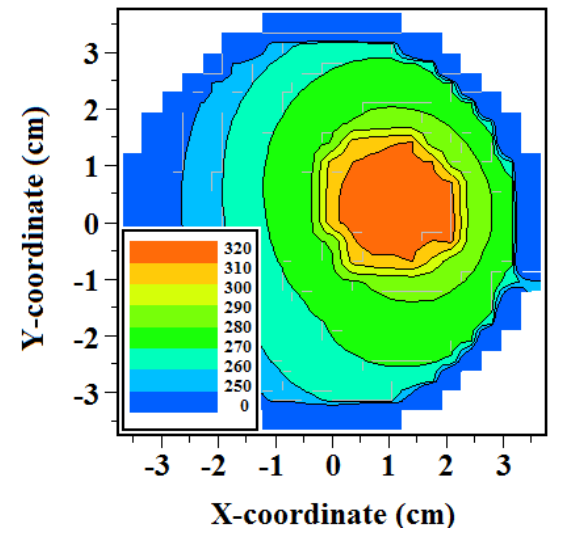

(a)

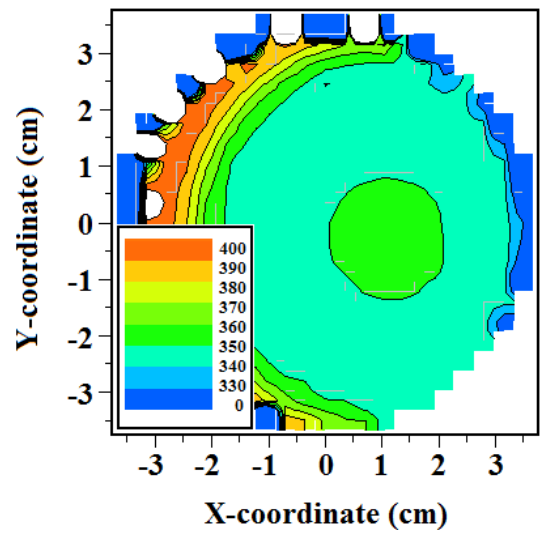

(b)

Figure 3.30. Surface map of AlN films with a nominal thickness of $0.3 \mu \mathrm{m}$. (a) undoped and (b) Er-doped. 


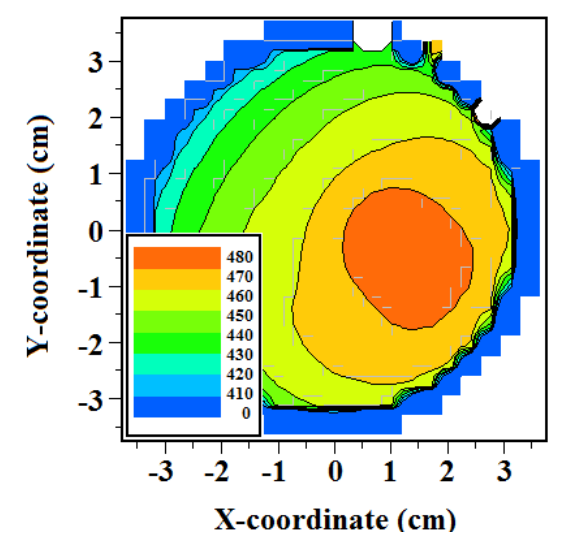

(a)

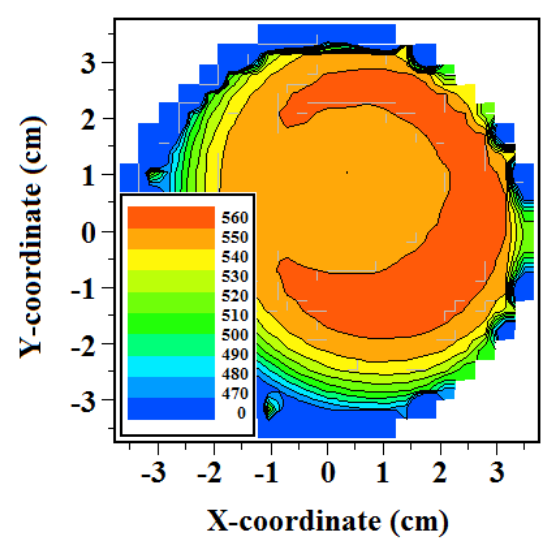

(b)

Figure 3.30. Surface map of AlN films with a nominal thickness of $0.5 \mu \mathrm{m}$. (a) undoped and (b) Er-doped.

\subsubsection{SEM}

The cross sections of the films were acquired using scanning electron microscopy (SEM). The images were taken to verify the thicknesses determined with spectroscopic ellipsometry. Since the AIN were insulating and were not coated with a metal prior to placing them in the SEM chamber, they experienced a moderate amount of surface charging. This led to image drift which increased the uncertainty of the thicknesses determined by SEM. In some cases, the SEM overestimated the film thickness (if the drift was in the direction of the scan) or underestimated the film thickness (if the drift was in a direction opposite to the direction of the scan). So, the thickness determined using SEM may not reflect the true thickness of the film. The SEM images of the undoped AIN films are shown in Figures 3.31(a)-3.33(a) and the SEM images of the Er-doped films are shown in Figures 3.31(b)-3.33(b). 


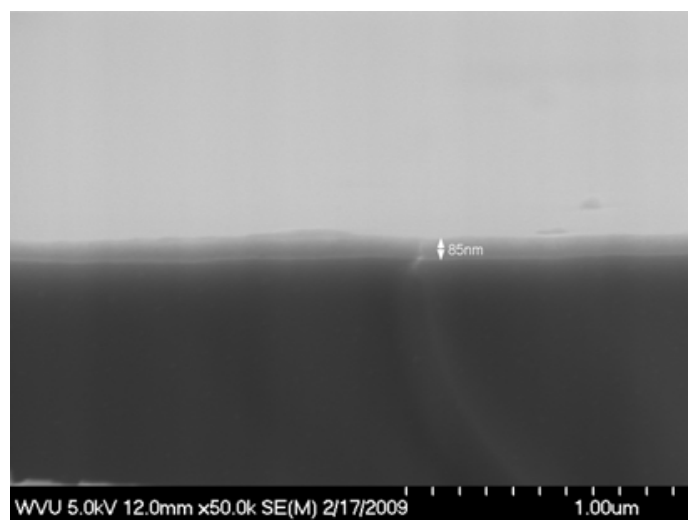

(a)

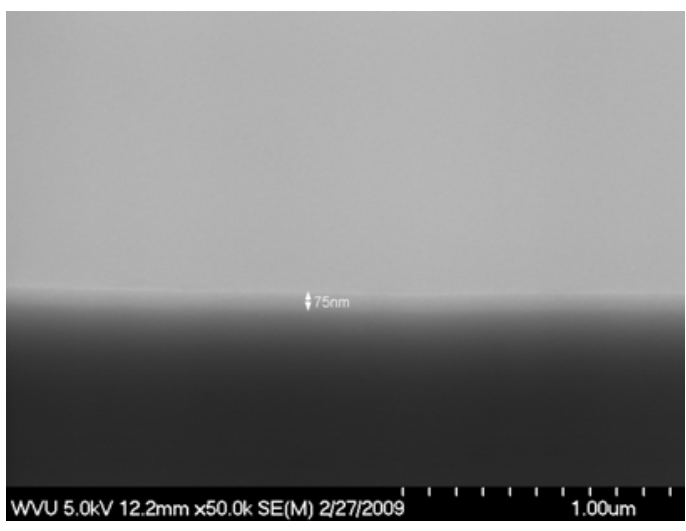

(b)

Figure 3.31. Cross-sectional SEM images of AlN films with a nominal thickness of $0.1 \mu \mathrm{m}$. (a) undoped $(t \approx 85 \mathrm{~nm})$ and (b) Er-doped $(t \approx 75 \mathrm{~nm})$.

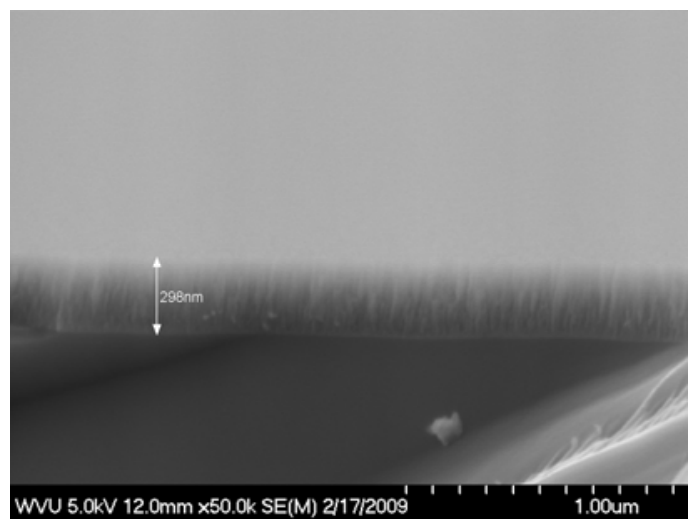

(a)

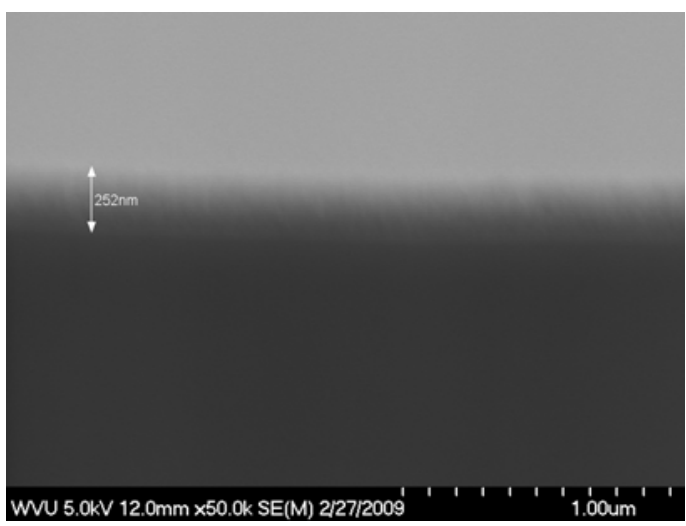

(b)

Figure 3.32. Cross-sectional SEM images of AlN films with a nominal thickness of $0.3 \mu \mathrm{m}$. (a) undoped $(t \approx 298 \mathrm{~nm})$ and (b) Er-doped $(t \approx 252 \mathrm{~nm})$. 


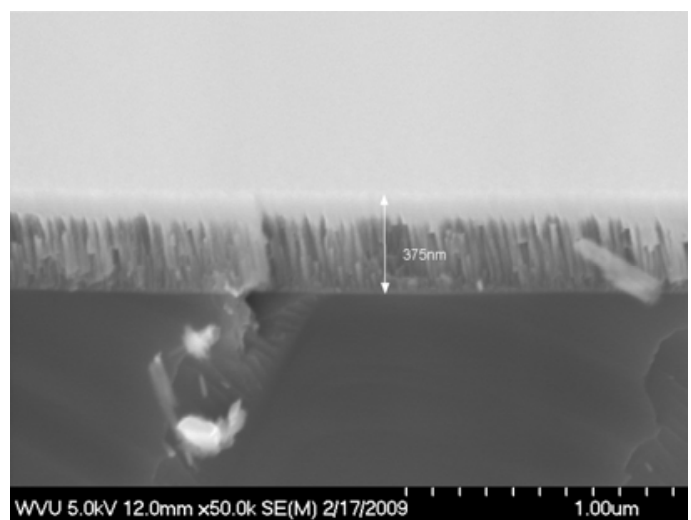

(a)



(b)

Figure 3.33. Cross-sectional SEM images of AlN films with a nominal thickness of $0.5 \mu \mathrm{m}$. (a) undoped $(t \approx 375 \mathrm{~nm})$ and (b) Er-doped $(t \approx 274 \mathrm{~nm})$.

The cross-sectional thicknesses measured using SEM supported the thicknesses determined using ellipsometry for the undoped films and the $0.1 \mu \mathrm{m}$ Er-doped film. (Note that the cross-sectional images were taken close to the edge of the wafer and so they are expected to be thinner than the nominal thickness.) The SEM indicated that the film thickness at the edge of the $0.3 \mu \mathrm{m}$ Er-doped film was $\sim 250 \mathrm{~nm}$ while the ellipsometry surface map indicated that it was $\sim 400 \mathrm{~nm}$ thick. In this case, the SEM measurements were assumed to be more accurate since they provided a direct measurement of the thickness. Additionally, it was anticipated that the film thickness for the $0.3 \mu \mathrm{m}$ Er-doped film should be between 200 and $300 \mathrm{~nm}$. Thus, the model used to fit the $0.3 \mu \mathrm{m}$ Er-doped film did not accurately represent the film. The same argument can be made for the $0.5 \mu \mathrm{m}$ Er-doped AlN film.

\subsubsection{AFM}

The surface roughness and grain height of the films were determined using tappingmode Atomic Force Microscopy (AFM). A Veeco multimode scanning probe was used to perform the measurements. The scan rate, scan size, number of samples, and data scale were kept constant for all of the measurements and were $0.25 \mathrm{~Hz}, 2 \mu \mathrm{m}, 512,25 \mathrm{~nm}$, 
respectively. Figures 3.34(a)-3.36(a) show the topography of the undoped AlN films and Figures 3.34(b)-3.36(b) show the topography of the Er-doped films.



(a)

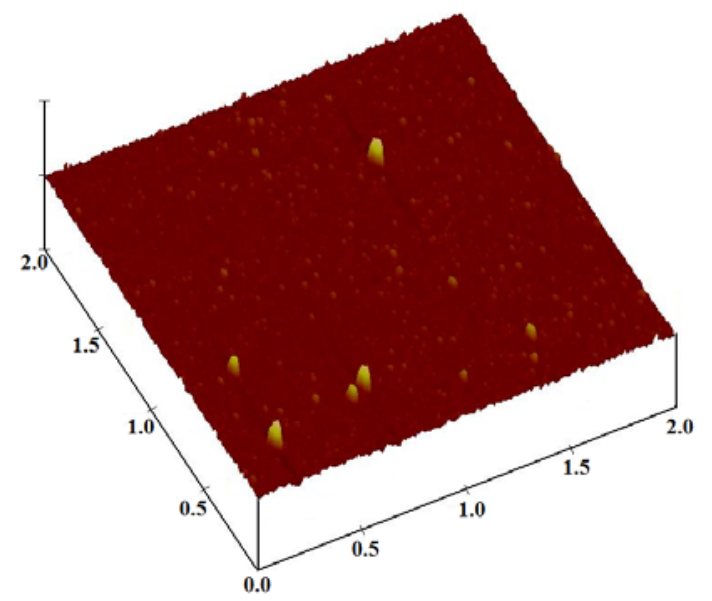

(b)

Figure 3.34. Topography of AlN films with a nominal thickness of $0.1 \mu \mathrm{m}$. (a) undoped and (b) Er-doped.

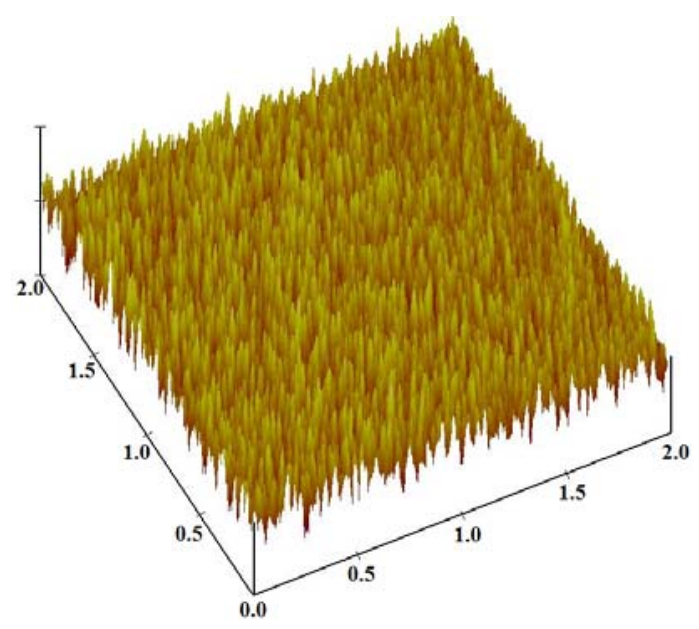

(a)

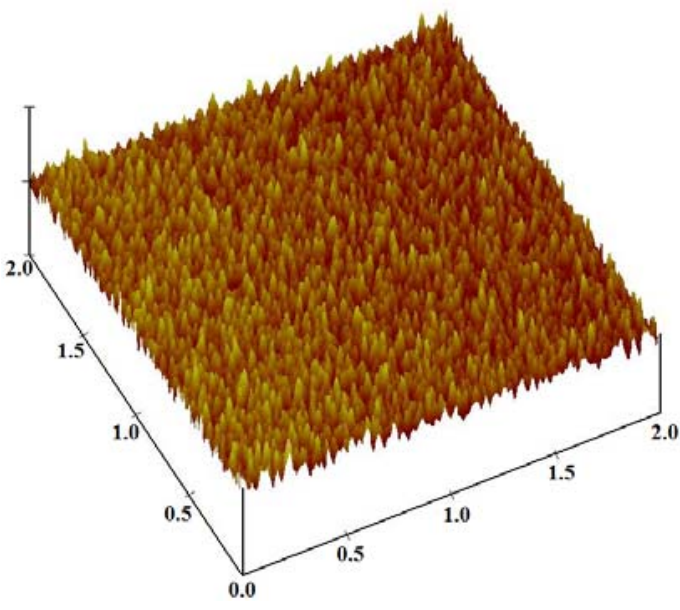

(b)

Figure 3.35. Topography of AlN films with a nominal thickness of $0.3 \mu \mathrm{m}$. (a) undoped and (b) Er-doped. 


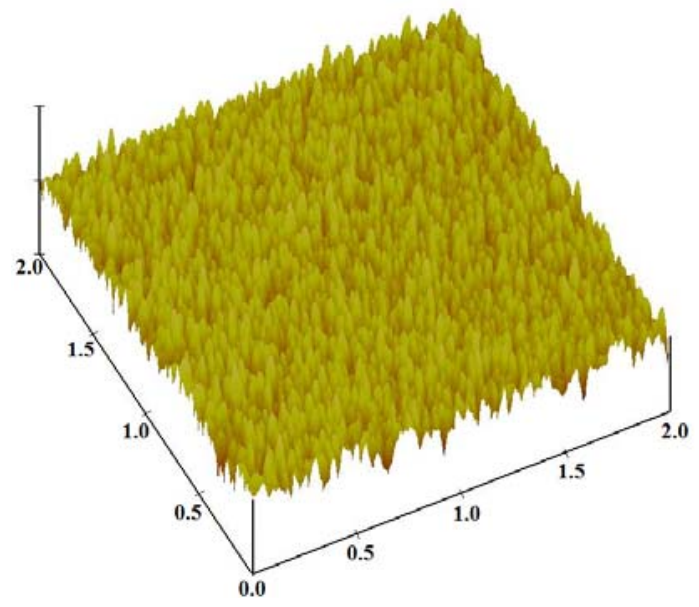

(a)

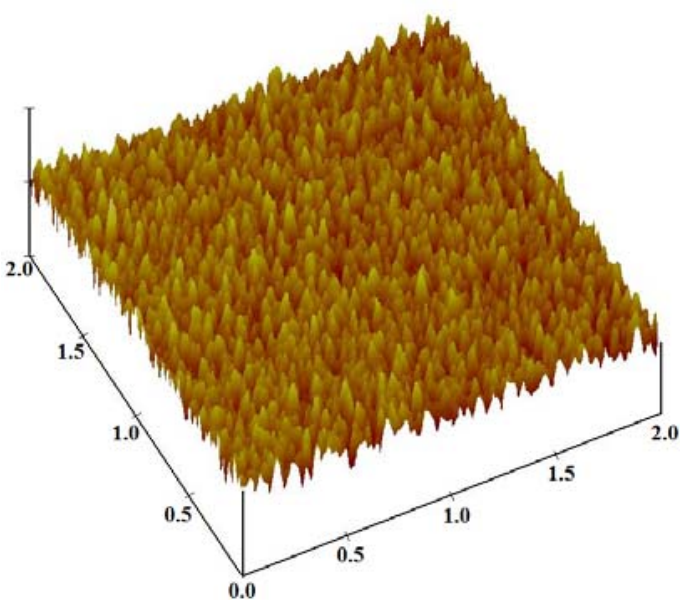

(b)

Figure 3.36. Topography of AlN films with a nominal thickness of $0.5 \mu \mathrm{m}$. (a) undoped and (b) Er-doped.

Using the Nanoscope software provided with the AFM system, the surface roughness and the grain height of the undoped AIN films were determined to be slightly larger than those of the Er-doped AlN films as shown in Figure 3.37 and Figure 3.38. In addition, the surface roughness for all of the films was less than $5 \mathrm{~nm}$. 


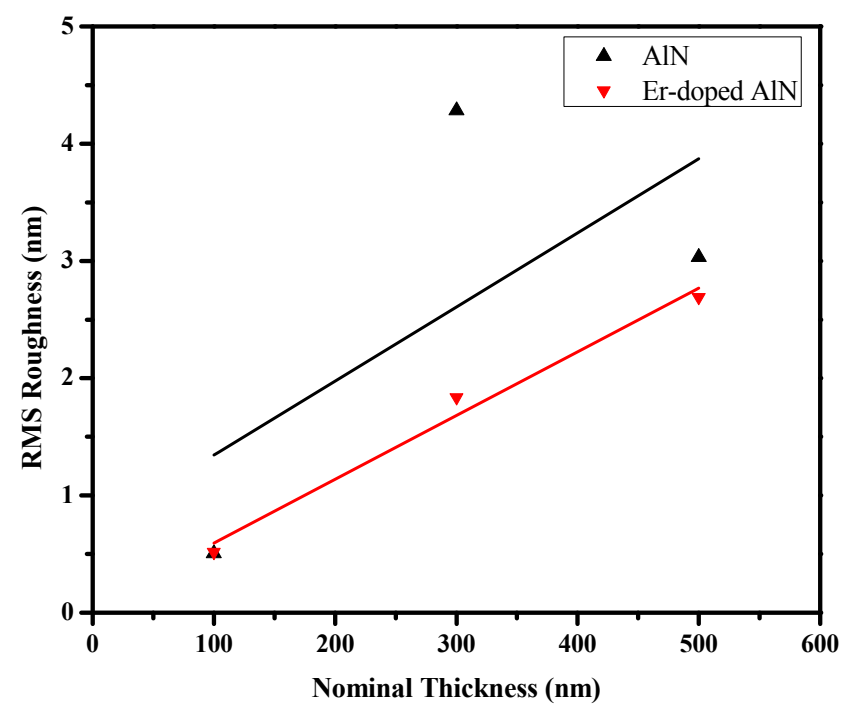

Figure 3.37. Measured surface roughness as a function of film thickness for undoped and Er-doped AlN films.

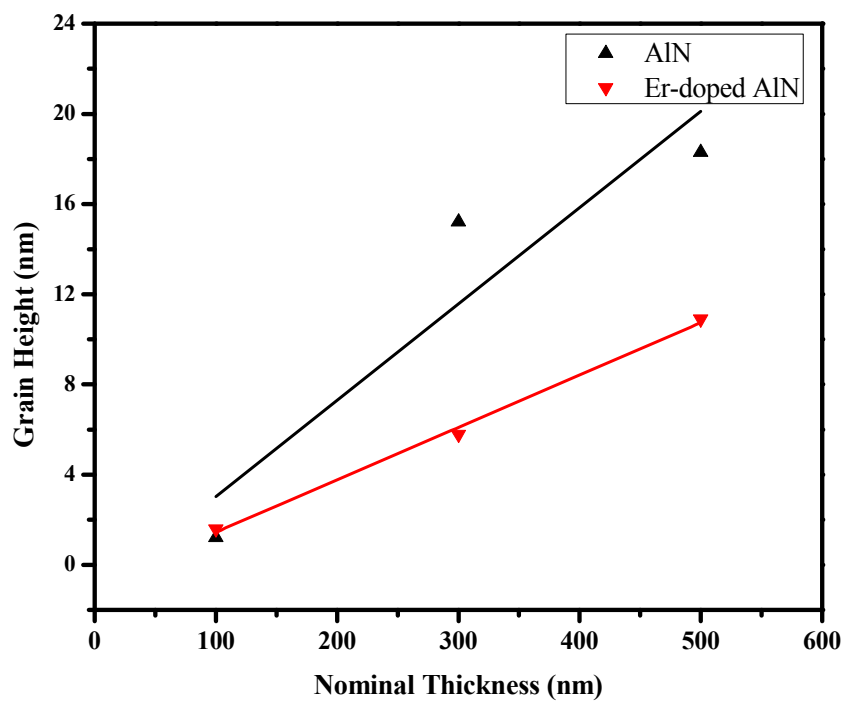

Figure 3.38. Measured grain height as a function of film thickness for undoped and Erdoped AlN films. 


\subsubsection{XPS}

XPS was performed on undoped and Er-doped AIN using a PHI 5000 Versa Probe XPS/UPS system. For all the scans performed in this work, the aluminum anode was used. A survey scan was performed prior to acquiring a detailed scan or a depth profile. For the survey scans, the X-ray spot size, power, and beam energy were typically set to $200 \mu \mathrm{m}, 50$ $\mathrm{W}$, and $15 \mathrm{kV}$, respectively. Additionally, the scan range, energy per step, pass energy, time per step, and average number of spectra were set to $0-1400 \mathrm{eV}, 0.500 \mathrm{eV}, 58.700 \mathrm{eV}$, $30 \mathrm{~ms}$, and 3. The scans were analyzed using the PHI MultiPak V8.2C software that came with the XPS system. Although neutralization was used in all scans, the spectra were typically shifted in energy by a small amount due to charging. This was corrected by shifting the spectra such that the $\mathrm{C} 1 \mathrm{~s}$ adventitious carbon peak was positioned at a binding energy of $284.8 \mathrm{eV}$. The survey scans for the undoped $0.1 \mu \mathrm{m}, 0.3 \mu \mathrm{m}$, and $0.5 \mu \mathrm{m}$ undoped AlN films are shown in Figures 3.39-3.41 and the survey scans for the corresponding Er-doped films are shown in Figures 3.42-3.44.



Figure 3.39. XPS survey scan of an undoped AlN film with a nominal thickness of $0.1 \mu \mathrm{m}$. 


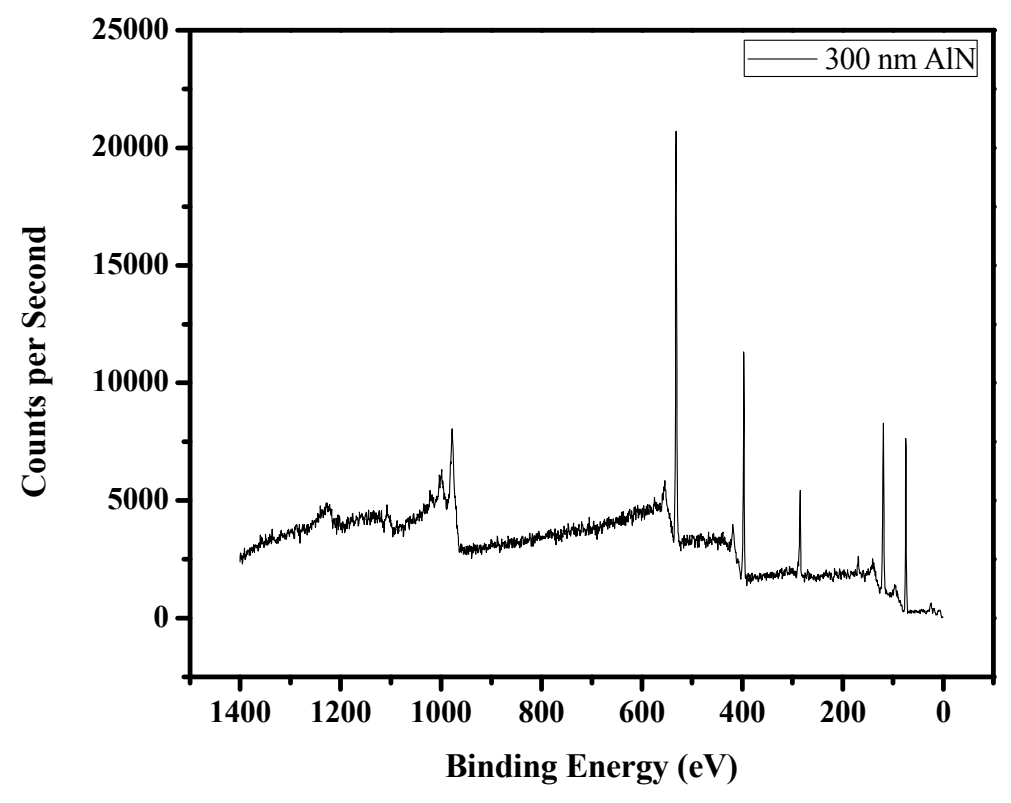

Figure 3.40. XPS survey scan of an undoped AlN film with a nominal thickness of $0.3 \mu \mathrm{m}$.

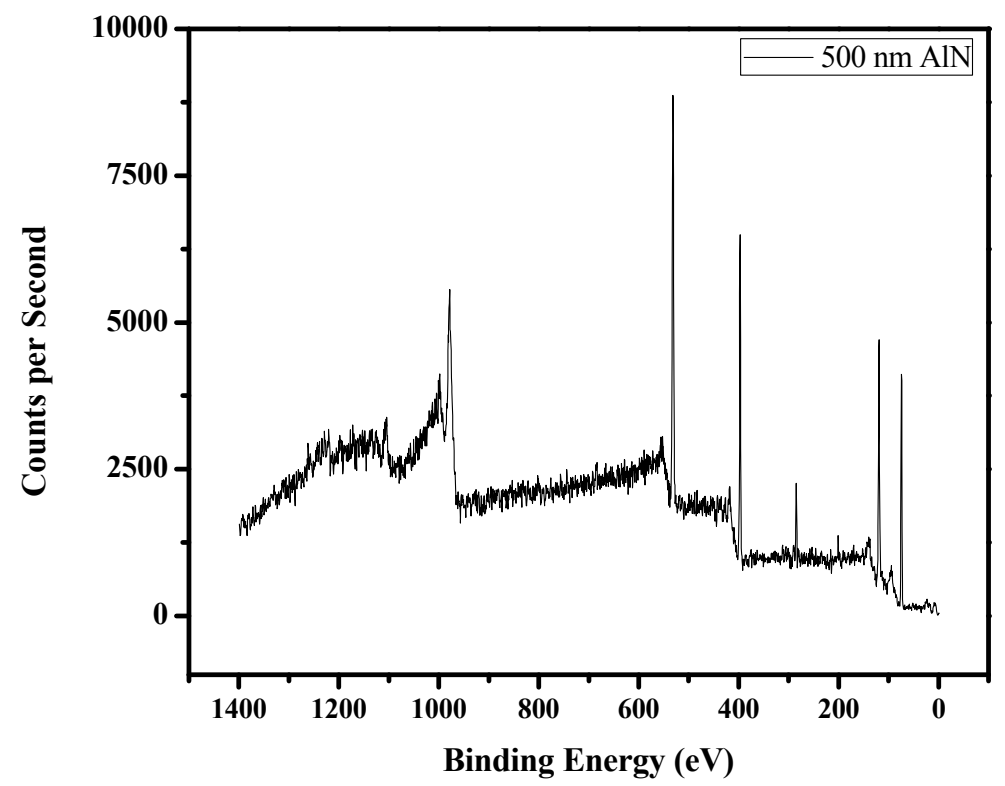

Figure 3.41. XPS survey scan of an undoped AlN film with a nominal thickness of $0.5 \mu \mathrm{m}$. 


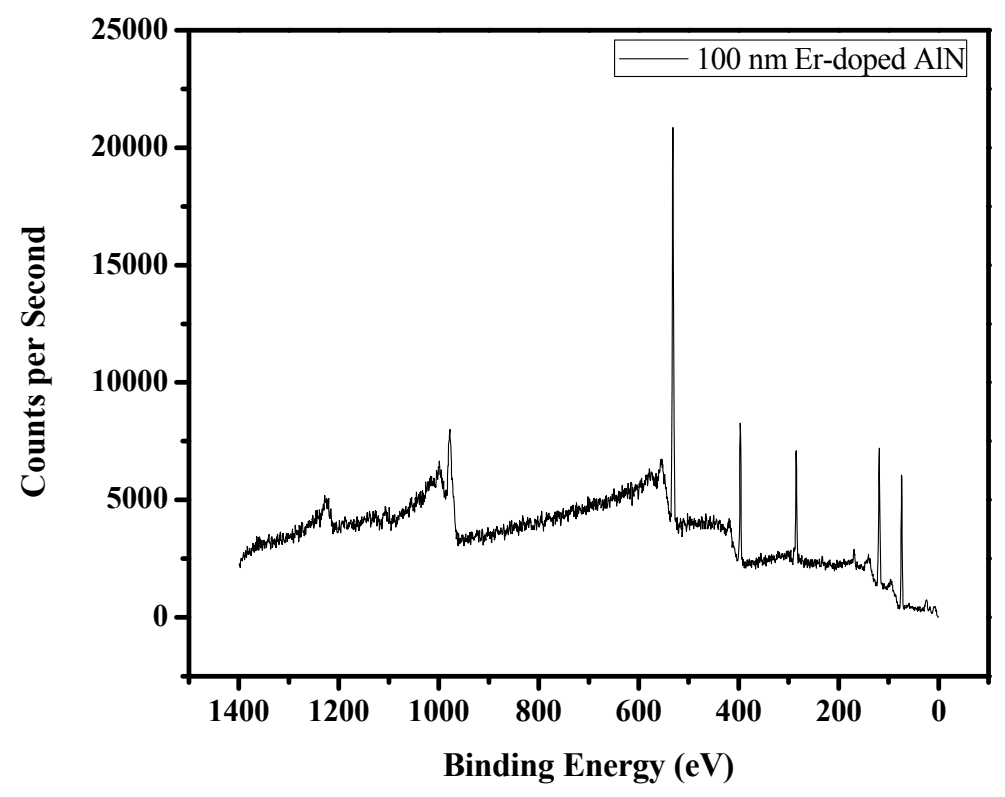

Figure 3.42. XPS survey scan of an Er-doped AlN film with a nominal thickness of $0.1 \mu \mathrm{m}$.

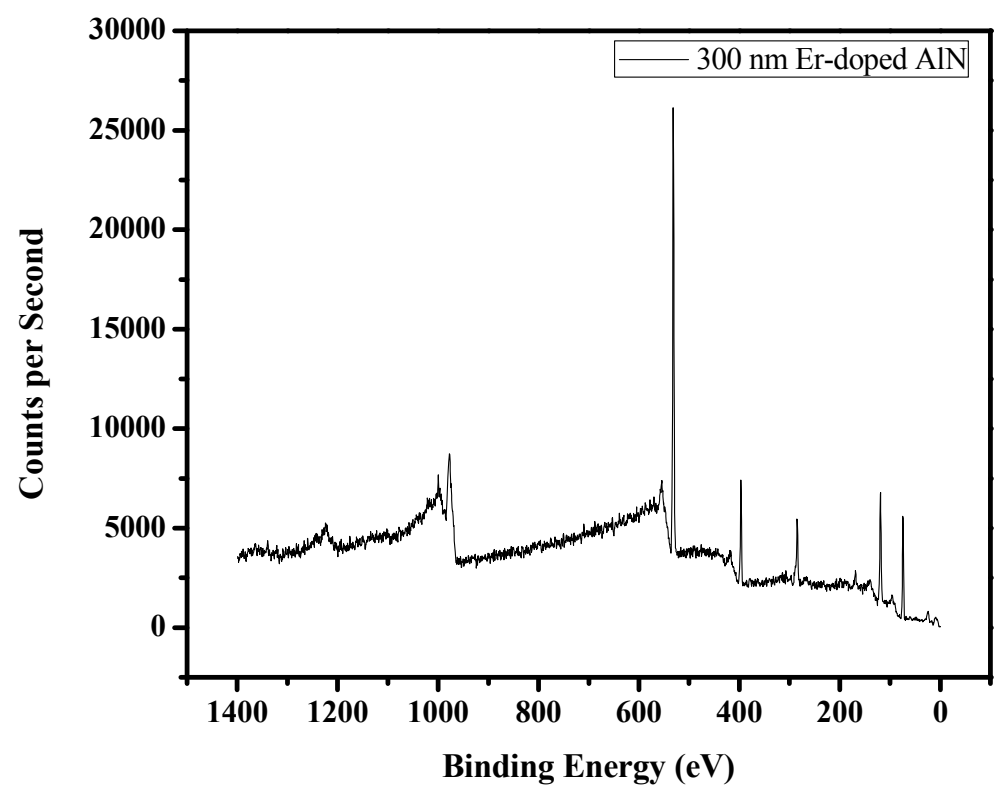

Figure 3.43. XPS survey scan of an Er-doped AlN film with a nominal thickness of $0.3 \mu \mathrm{m}$. 


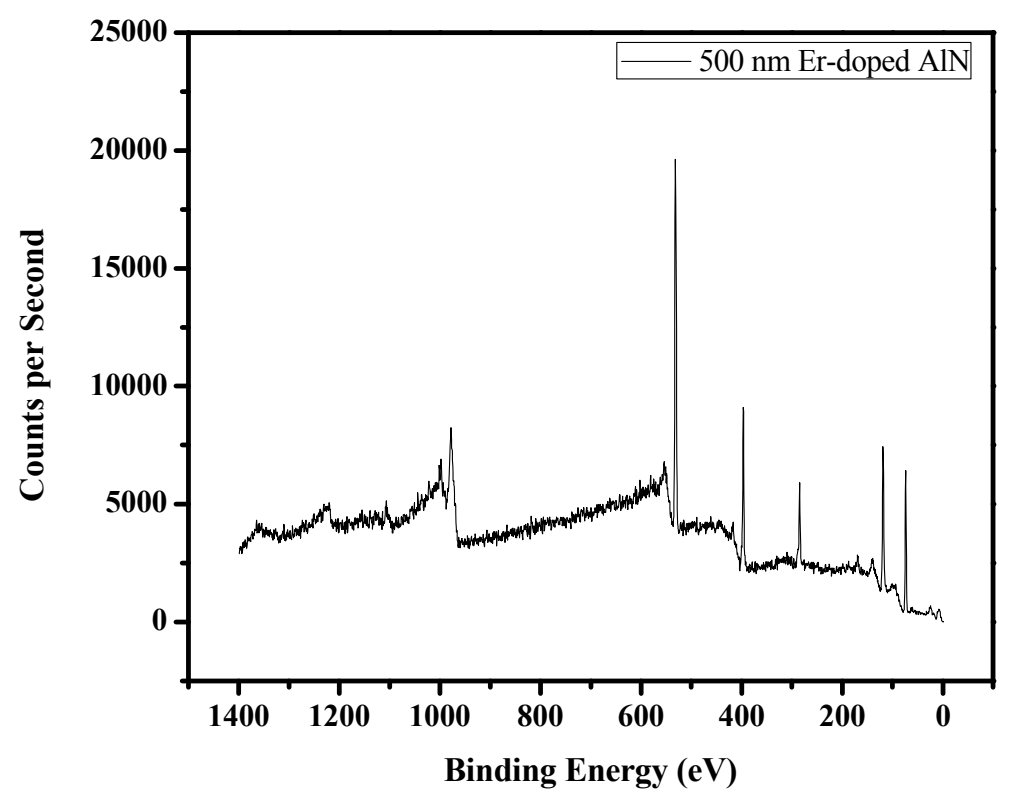

Figure 3.44. XPS survey scan of an Er-doped AlN film with a nominal thickness of $0.5 \mu m$.

In most cases, detailed scans for the $\mathrm{C} 1 \mathrm{~s}, \mathrm{O} 1 \mathrm{~s}, \mathrm{Si} 2 \mathrm{p}, \mathrm{Al} 2 \mathrm{p}, \mathrm{N} 1 \mathrm{~s}$, and Er4d peaks were acquired. For these scans, the X-ray spot size, power, and beam energy were typically set to $200 \mu \mathrm{m}, 50 \mathrm{~W}$, and $15 \mathrm{kV}$, respectively. The scan ranges were set to 276 $296 \mathrm{eV}, 522-540 \mathrm{eV}, 93-111 \mathrm{eV}, 65-83 \mathrm{eV}, 390-410 \mathrm{eV}$, and 160-180 eV for the previously listed peaks. The average number of spectra was set to 2 for all the detailed scans except for the erbium detailed scan in which case it was set to 16. Additionally, the energy per step, pass energy, and time per step were set to $0.050 \mathrm{eV}, 58.700 \mathrm{eV}$, and $50 \mathrm{~ms}$. The scans were analyzed using the PHI MultiPak V8.2C software that came with the XPS system. Although neutralization was used in all scans, the spectra were typically shifted in energy by a small amount due to charging. This was corrected by shifting the spectra such that the C1s adventitious carbon peak was positioned at a binding energy of $284.8 \mathrm{eV}$.

The atomic concentrations were determined by fitting the detailed scan peaks. For all of the films, the aluminum-to-nitrogen ratio was approximately 1:1. Additionally, all the films had a high atomic concentration of oxygen between 15 and 20 atomic percent. All films were found to have a thin layer of adsorbed carbon contamination on their 
surfaces. The adventitious carbon contamination was used to determine if the spectra was shifted. After sputtering the sample with an ion argon gun for a short time, the $\mathrm{C} 1 \mathrm{~s}$ peak disappeared entirely indicating that the carbon contamination was only in a thin film on the surface. The nitrogen peaks showed that most of the nitrogen in the films was chemically bonded to aluminum. In all films, a small peak at $\sim 169 \mathrm{eV}$ was found. This is unexpected since the Er4d peak at $169 \mathrm{eV}$ should only be present in the Er-doped films. More work is needed to investigate the origin of these peaks.

All of the films were also depth profiled. The sputter rate for reactively sputtered AlN was found experimentally to be $\sim 2.4 \mathrm{~nm} /$ min when using the " $1 \mathrm{kV}-2 \times 2$ Max" ion gun setting. The depth profile was obtained by sputtering the sample and acquiring the data repetitively. The $\mathrm{C} 1 \mathrm{~s}, \mathrm{O} 1 \mathrm{~s}, \mathrm{Si} 2 \mathrm{p}, \mathrm{Al} 2 \mathrm{p}, \mathrm{N} 1 \mathrm{~s}$, and Er4d peaks were acquired using the "unscan" mode. The pass energy, range, energy per step, and time per step were set to $117.400 \mathrm{eV}, 15.009 \mathrm{eV}, 1.001 \mathrm{eV}$ and $50 \mathrm{~ms}$, respectively, for all of the depth profile scans. The scan start energies were set to $278 \mathrm{eV}, 523 \mathrm{eV}, 92 \mathrm{eV}, 68 \mathrm{eV}, 391 \mathrm{eV}$, and $160 \mathrm{eV}$ for the previously listed peaks. The average number of spectra was set to 2 for all of the peaks except for the erbium peak in which case it was set to 9. The depth profiles of undoped AlN films are shown in Figures 3.45-3.47 and those of the Er-doped AlN films are shown in Figures 3.48-3.50. 


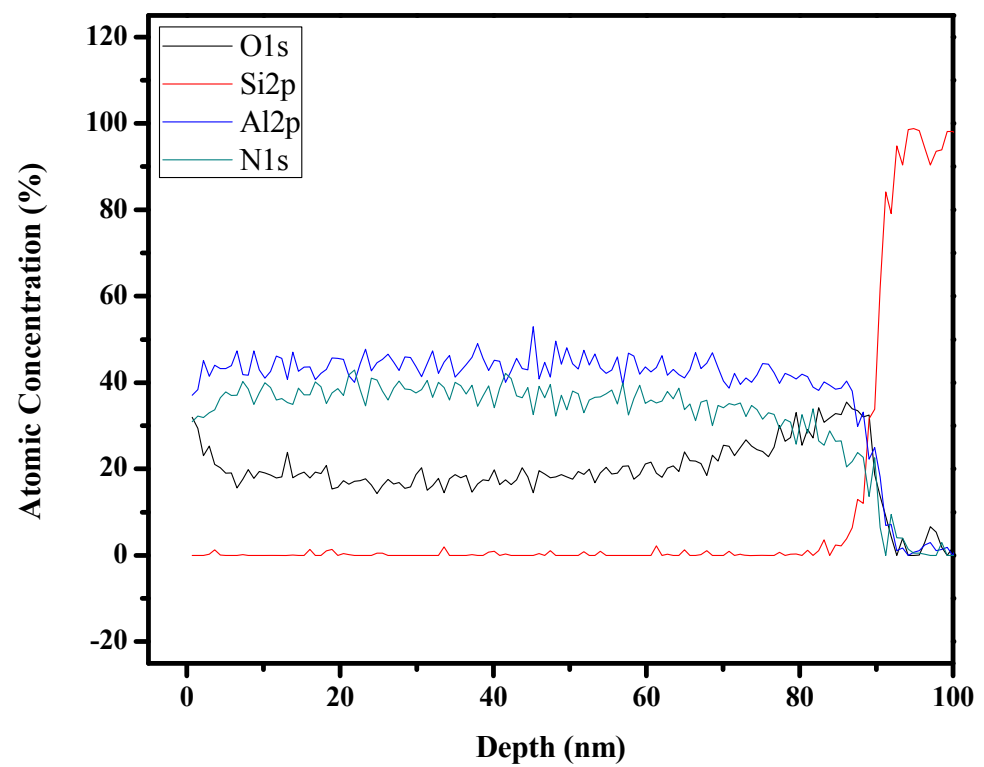

Figure 3.45. XPS depth profile of an undoped AlN film with a nominal thickness of $0.1 \mu \mathrm{m}$.

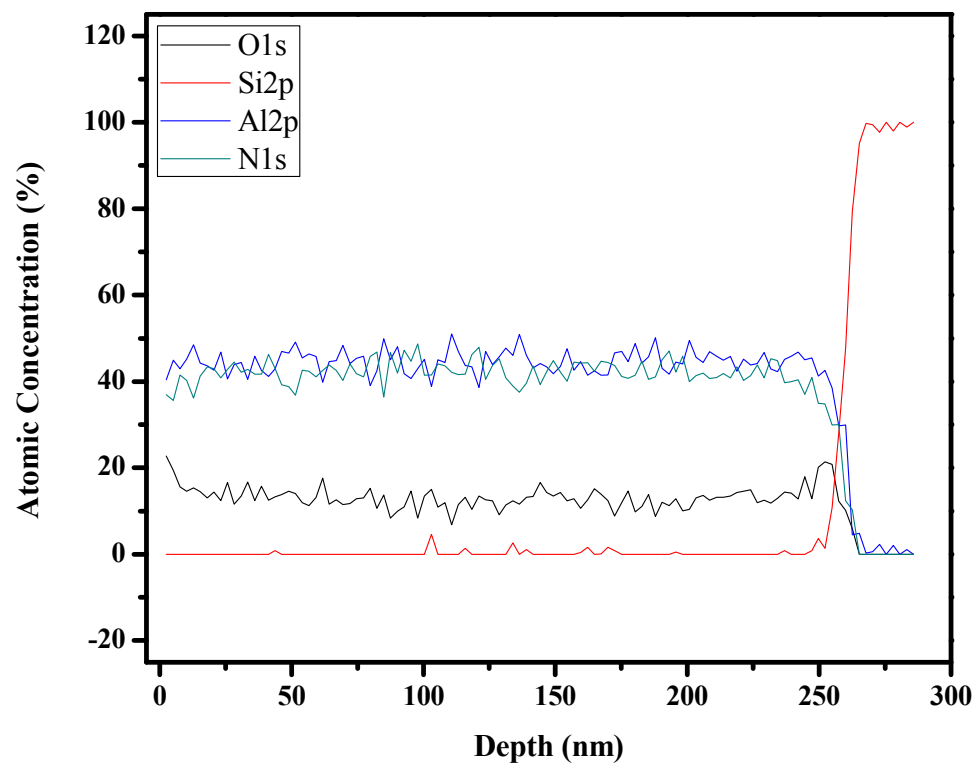

Figure 3.46. XPS depth profile of an undoped AlN film with a nominal thickness of $0.3 \mu \mathrm{m}$. 




Figure 3.47. XPS depth profile of an undoped AlN film with a nominal thickness of $0.5 \mu m$. (Note: the number of data points was shown in the plot was reduced by a factor of 3 in $M A T L A B)$

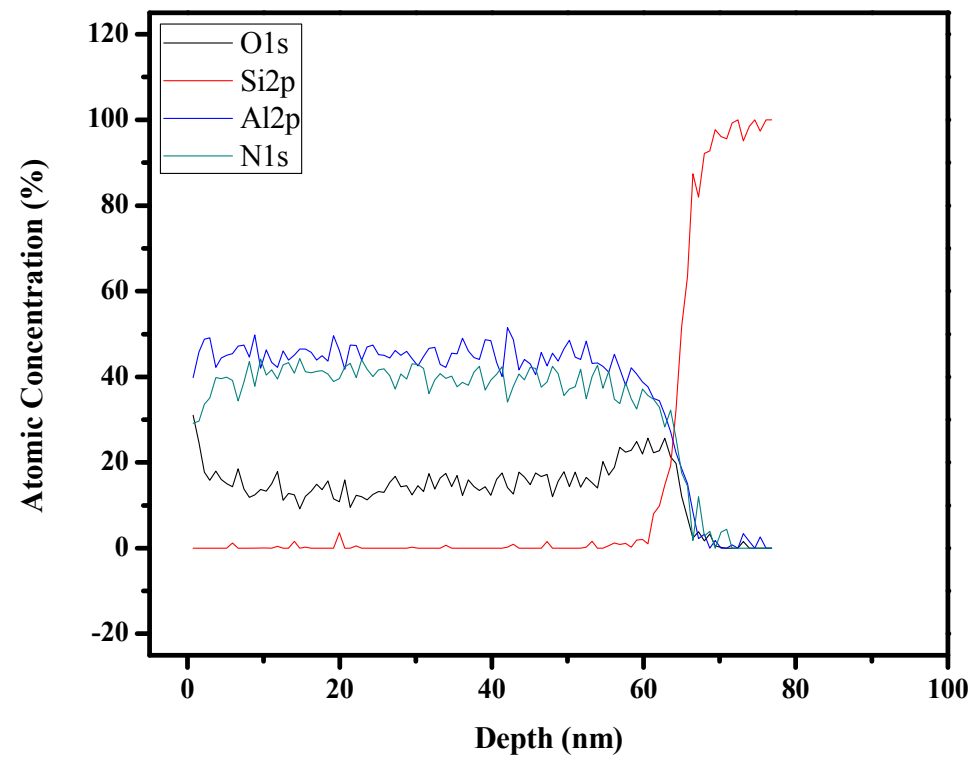

Figure 3.48. XPS depth profile of an Er-doped AlN film with a nominal thickness of 0.1 $\mu m$. 




Figure 3.49. XPS depth profile of an Er-doped AlN film with a nominal thickness of 0.3 $\mu m$.

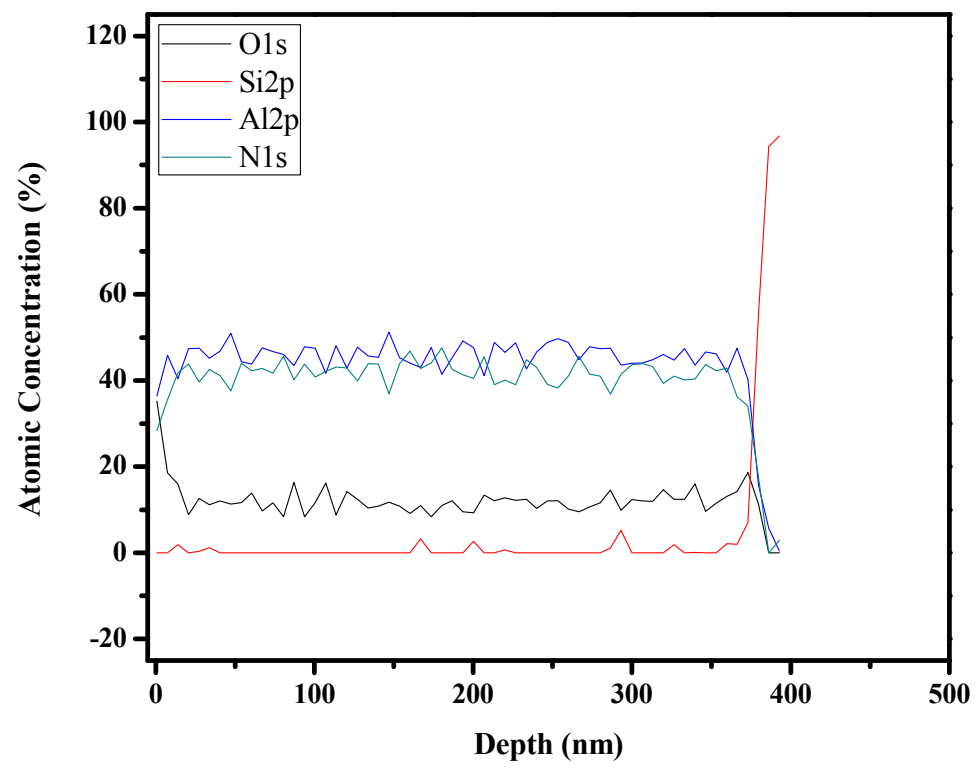

Figure 3.50. XPS depth profile of an Er-doped AlN film with a nominal thickness of 0.5 $\mu \mathrm{m}$. (Note: the number of data points was shown in the plot was reduced by a factor of 3 in $M A T L A B)$ 
As shown in the plots, the aluminum, nitrogen and oxygen concentrations were relatively constant through most of the film. However, all films showed a higher concentration of oxygen near their surface (see Section 2.3.2). Additionally, a slight increase in oxygen concentration was also noticed near the silicon interface indicating a thin layer of silicon dioxide.

\subsubsection{SUMMARY}

In this work, undoped and Er-doped films with thicknesses of $0.1 \mu \mathrm{m}, 0.3 \mu \mathrm{m}$, and $0.5 \mu \mathrm{m}$ were characterized using ellipsometry, AFM, SEM, and XPS. The Er-doped films were found to be slightly smoother and to have a lower grain height. The Cauchy dispersion model fit the undoped AIN films extremely well leading to accurate determinations of the film thicknesses, among others. In contrast, the Cauchy dispersion model only fit the $0.1 \mu \mathrm{m}$ Er-doped AIN film well and fit the $0.3 \mu \mathrm{m}$ and the $0.5 \mu \mathrm{m} \mathrm{Er-}$ doped AlN films poorly. This could be determined by noting the ratios of the MSE to the film thickness which was greater than $10 \%$ for the two thicker Er-doped films. SEM further invalidated the models used for the $0.3 \mu \mathrm{m}$ and $0.5 \mu \mathrm{m}$ Er-doped AlN films by giving a much lower film thickness for these films. In addition, XPS was used to determine the elemental composition of the films. It was found that the ratio of aluminumto-nitrogen was almost perfect in all cases. However, oxygen concentrations of $15-20 \%$ were observed in all the films. All of the films were found to have a larger oxygen concentration at the surface of the films indicating oxidation. Detailed scans were performed to quantify the erbium incorporation in the Er-doped AIN films. However, a peak at $\sim 169 \mathrm{eV}$ was found in all of the films, so XPS could not be used to determine this quantity. Further investigation into the properties of these films using LDV, Raman, Fourier Transform Infrared spectroscopy (FTIR), and X-ray diffraction (XRD) is needed. Additionally, nano-indentation services provided by companies such as Hysitron could be ordered to determine if the mechanical properties are influenced by erbium incorporation. 


\subsubsection{AIN MIS STRUCTURES}

The effective piezoelectric coefficient of AIN MIS structures has been shown to be a function of contact metal work function [Harman, 2008]. High work function metals such as platinum resulted in the largest MIS structure displacement while low work function metals resulted in the smallest MIS structure displacement. It is believed that this is a result of the built-in electric fields generated in the MIS structure. Methods of enhancing the effective response of the films are very desirable for many pMEMS and SAW applications. In this work, $\mathrm{C}-\mathrm{V}$ measurements were performed to look for changes in the electrical properties of MIS structures differing only in the contact metals used. In particular, four configurations were examined and were $\mathrm{Al} / \mathrm{AlN} / p-\mathrm{Si} / \mathrm{Al}, \mathrm{Pt} / \mathrm{AlN} / p-\mathrm{Si} /$ $\mathrm{Al}, \mathrm{Al} / \mathrm{AlN} / p$-Si/ Pt, and Pt/ AlN/ $p$-Si/ Pt. These configurations were chosen because

they provide the most Schottky contacts and the most Ohmic contacts to both the $p$-Si and the AlN layers.

\subsubsection{AIN DEPOSITION}

An AlN film with a nominal thickness of $1.0 \mu \mathrm{m}$ was reactively sputtered using a CVC 610 DC magnetron sputter deposition system. The substrate used was a (100) p-type, double-side polished, 1-20 $\Omega$-cm resistivity silicon wafer purchased from Wafer World, Inc. (www.waferworld.com). Prior to loading the wafer in the sputtering chamber, the wafer was cleaned in a 5 minute acetone bath, a 5 minute methanol bath, and a 5 minute dip in 10:1 deionized (DI) water to J.T. Baker $49 \%$ hydrofluoric acid (HF). The wafer was rinsed in a cascading bath of DI water for 10 minutes, blown dry with nitrogen gas, and baked in a gravity oven for 10 minutes at $110^{\circ} \mathrm{C}$.

A $50 \mathrm{~mm}$-diameter, $99.999 \%$ pure Al target was to deposit the AlN film. Prior to deposition, the sputtering chamber was pumped to a base pressure below $5 \times 10^{-6}$ Torr using a CTI-8 cryopump. Since the Al target was known to oxidize when stored in an ambient environment, a two step pre-sputter was performed to remove the surface oxidation/contamination and to poison the target with a thin layer of AlN. The pre-sputter was immediately followed by film deposition. All pre-sputters and depositions were performed with a magnetron power of $100 \mathrm{~W}$ and a working pressure of $45 \mathrm{mT}$ Torr. 


\subsubsection{CONTACT DEPOSITION}

Circular contacts were deposited on the AlN film using image reversal photolithograpy. HexaMethylDiSilazane (HMDS) was spun onto the wafer for 30 seconds at $4000 \mathrm{rpm}$ to promote adhesion. Next, AZ5214-E photoresist was spun onto the wafer for 30 seconds at $4000 \mathrm{rpm}$ to form a $1.4 \mu \mathrm{m}$ thick layer of photoresist on the surface of the AlN film. The wafer was placed on a hotplate for 30 seconds at $95^{\circ} \mathrm{C}$ to remove excess solvents in the film. Once the photoresist was dried enough not to be sticky, the wafer was loaded into a Karl Suss MA-6 mask aligner and was exposed to ultraviolet light through a mask with circles having radii of $400 \mu \mathrm{m}$. To perform image reversal, the exposed wafer was placed on a hotplate for 120 seconds at $120^{\circ} \mathrm{C}$ and then it was exposed under an ultraviolet flood lamp for 82 seconds. The photoresist was developed in MIF-300 developer, rinsed in deionized water, and blown dry in nitrogen gas. The wafer was loaded into the sputtering chamber and either platinum or aluminum was sputtered on the patterned side. The wafer was removed from the chamber and placed in a bath of acetone to perform lift-off. The remaining photoresist dissolved in the solvent leaving the circular metal contacts behind. The wafer was cleaned in a 5 minute bath in acetone and then a 5 minute bath in methanol and was blown dry in nitrogen gas. The backside contact was deposited by sputtering the desired metal ( $\mathrm{Pt}$ or $\mathrm{Al})$ directly onto the bare silicon wafer.

\subsubsection{LDV}

The piezoelectric responses of the AlN MIS structures were determined using laser Doppler vibrometry (LDV). As reported previously, the MIS structures having platinum contacts had a higher effective piezoelectric response than MIS structure having aluminum contacts.

\subsubsection{C-V}

C-V measurements were on the AIN MIS structures using an Agilent E4980A precision LCR meter, a Signatone S-1160B-5N probe station, and two Signatone S-745CxM probes. Measurements were taken at $3.5 \mathrm{kHz}$ and $1 \mathrm{MHz}$. The test level, start bias, 
end bias, and step were set in a MATLAB m-file to $100 \mathrm{mV},-40 \mathrm{~V}$ (or $40 \mathrm{~V}$ ), $40 \mathrm{~V}$ (or $40 \mathrm{~V}$ ), and $1 \mathrm{~V}$ (or $-1 \mathrm{~V}$ ), respectively. Typical $\mathrm{C}-\mathrm{V}$ curves generated at $1 \mathrm{MHz}$ for the $\mathrm{Al} /$ $\mathrm{AlN} / p$-Si/ $\mathrm{Al}, \mathrm{Pt} / \mathrm{AlN} / p$-Si $/ \mathrm{Al}, \mathrm{Al} / \mathrm{AlN} / p$-Si/ $\mathrm{Pt}$, and $\mathrm{Pt} / \mathrm{AlN} / \mathrm{p}-\mathrm{Si} / \mathrm{Pt}$ MIS structures are shown in Figures 3.51-3.54. The measurements at $3.5 \mathrm{kHz}$ were taken because this frequency matched the one typically used for LDV measurements. However, the corresponding plots did not show much change in capacitance as a function of voltage (flat $\mathrm{C}-\mathrm{V}$ line) and so these plots are not shown.



Figure 3.51. Typical C-V curve of an Al/ AlN/p-Si/Al structure with a $1 \mu \mathrm{m}$ AlN insulating layer. 


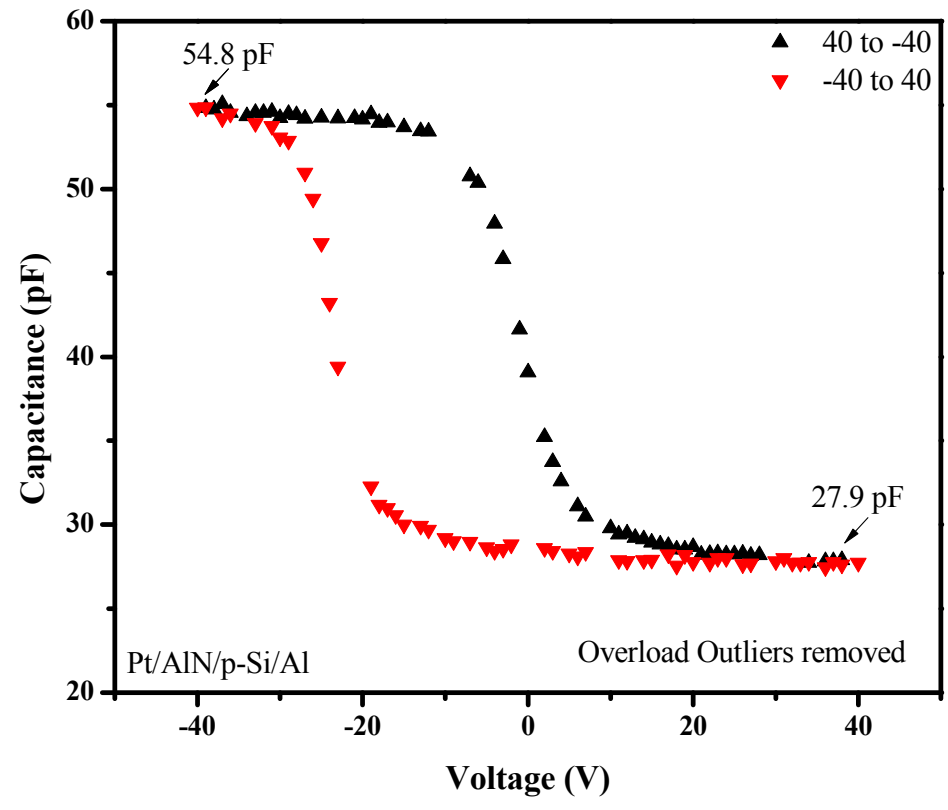

Figure 3.52. Typical C-V curve of a Pt/ AlN/p-Si/ Al structure with a $1 \mu \mathrm{m}$ AlN insulating layer.

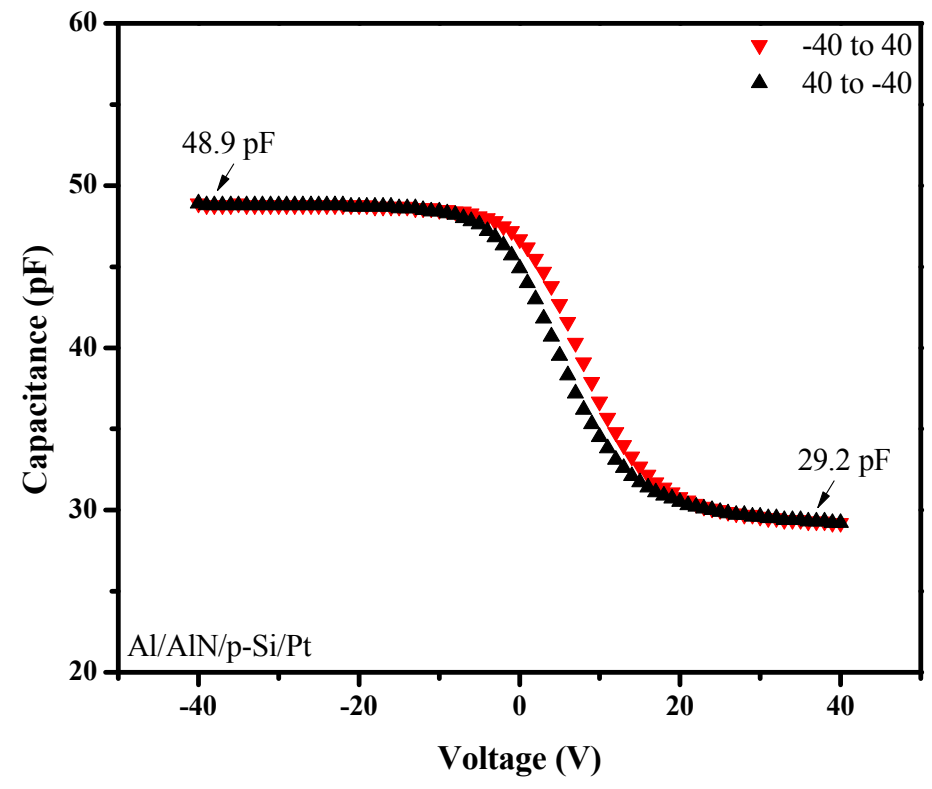

Figure 3.53. Typical C-V curve of an Al/ AlN/p-Si/ Pt structure with a $1 \mu \mathrm{m}$ AlN insulating layer. 


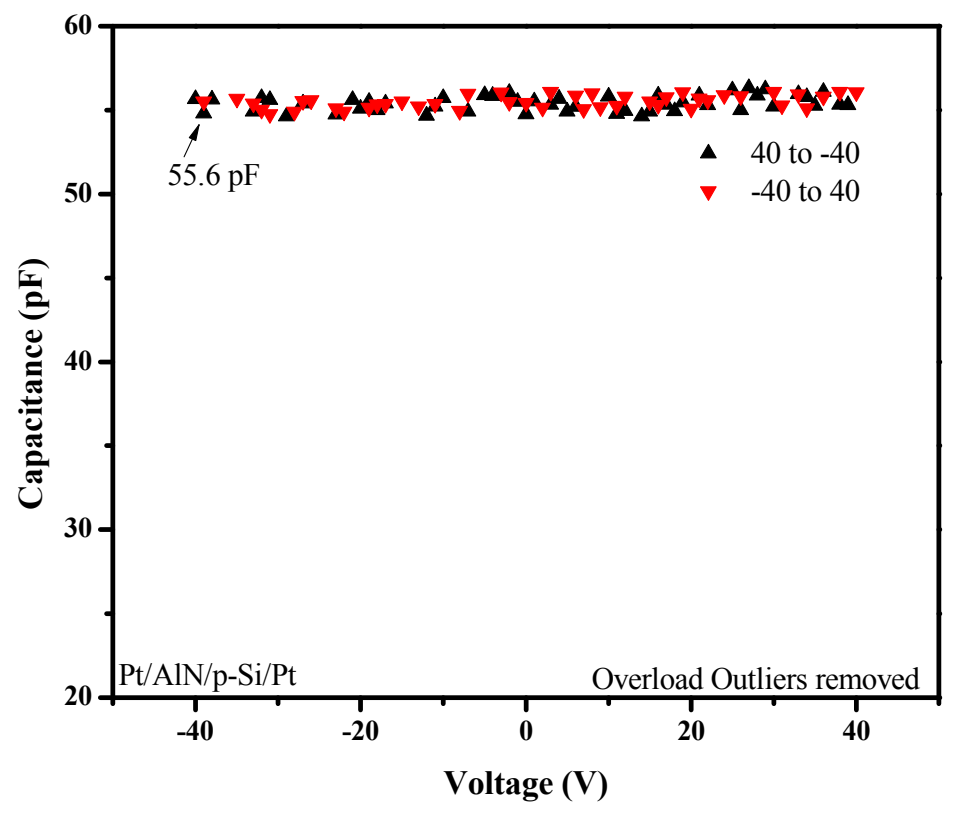

Figure 3.54. Typical C-V curve of a Pt/ AlN/p-Si/Pt structure with a $1 \mu \mathrm{m}$ AlN insulating layer.

In all cases except the $\mathrm{Pt} / \mathrm{AlN} / p$-Si/ Pt case, the high frequency $\mathrm{C}-\mathrm{V}$ curves had the expected characteristic shape. The hysteresis area (corresponding to trapped charge) was greatest for the MIS structures that had an aluminum backside contact. This was expected since aluminum forms a Schottky contact with $p$-Si. Thus, additional charge can accumulated at this interface. The $\mathrm{C}-\mathrm{V}$ curve for the $\mathrm{Pt} / \mathrm{AlN} / p-\mathrm{Si} / \mathrm{Al}$ case was the most ideal for the four cases. The $\mathrm{Pt} / \mathrm{AlN} / p-\mathrm{Si} / \mathrm{Pt}$ case did not show a voltage dependent capacitance. This was thought to be because the silicon contact was Ohmic and the AlN contact was Schottky. The stored charge and the effective piezoelectric response for the four configurations are shown in Figure 3.57. It is believed that the maximum internal electric field is developed when most of the applied voltage is applied across the AlN layer (i.e. Ohmic contact to the silicon substrate) and the highest Schottky barrier is formed at the metal/AlN interface (i.e. Schottky contact to the aluminum nitride film). 


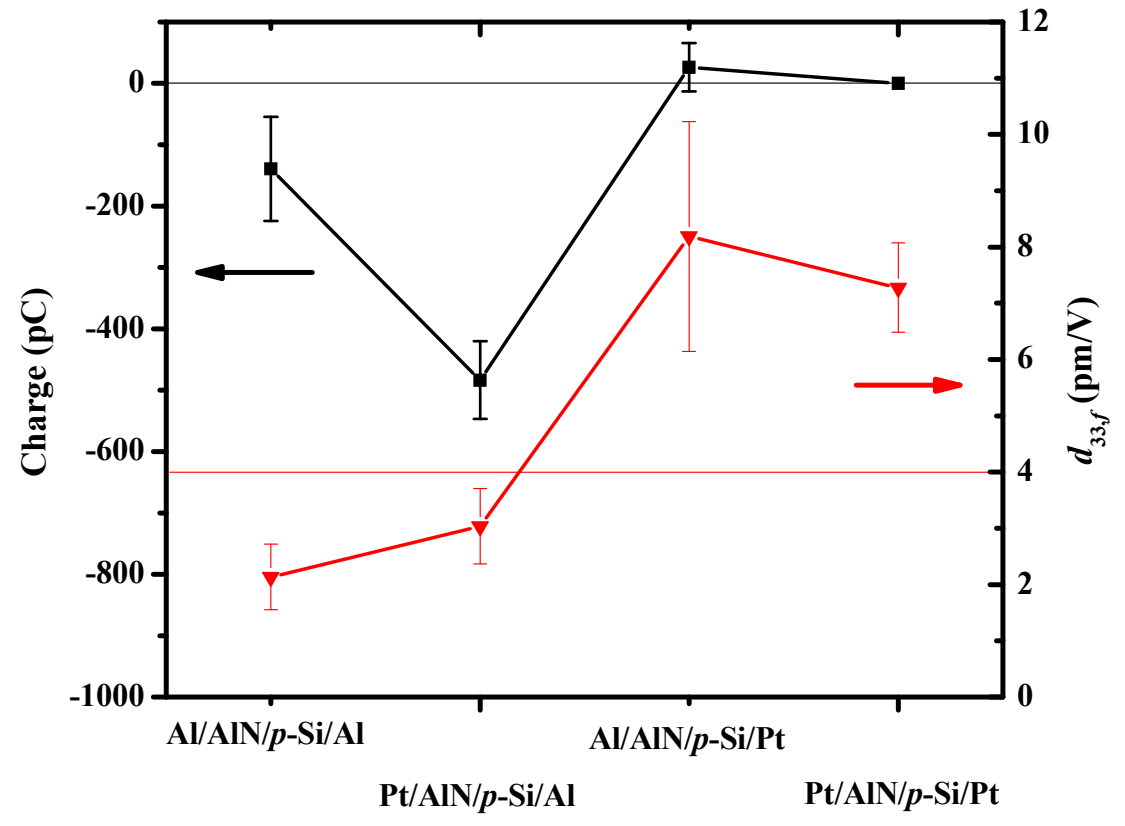

Figure 3.55. Measured charge and measured effective longitudinal piezoelectric coefficient for four types of MIS structures.

Thus, the effective piezoelectric response should be influenced by the applied dc offset. Applying a constant dc voltage is thought to have the effect of increasing or decreasing the magnitude of the internal electric field in the AIN layer. The plot of effective piezoelectric response verses applied dc bias is shown in Figure 3.56. More measurements should be performed to reduce the error bars in Figure 3.55. However, it is believed that the built-in electric field is much larger than the electric field induced in the AlN film when applying a de voltage between $-10 \mathrm{~V}$ and $10 \mathrm{~V}$. Thus, the dc bias was not found to strongly affect the piezoelectric response over the range of dc biases considered. 


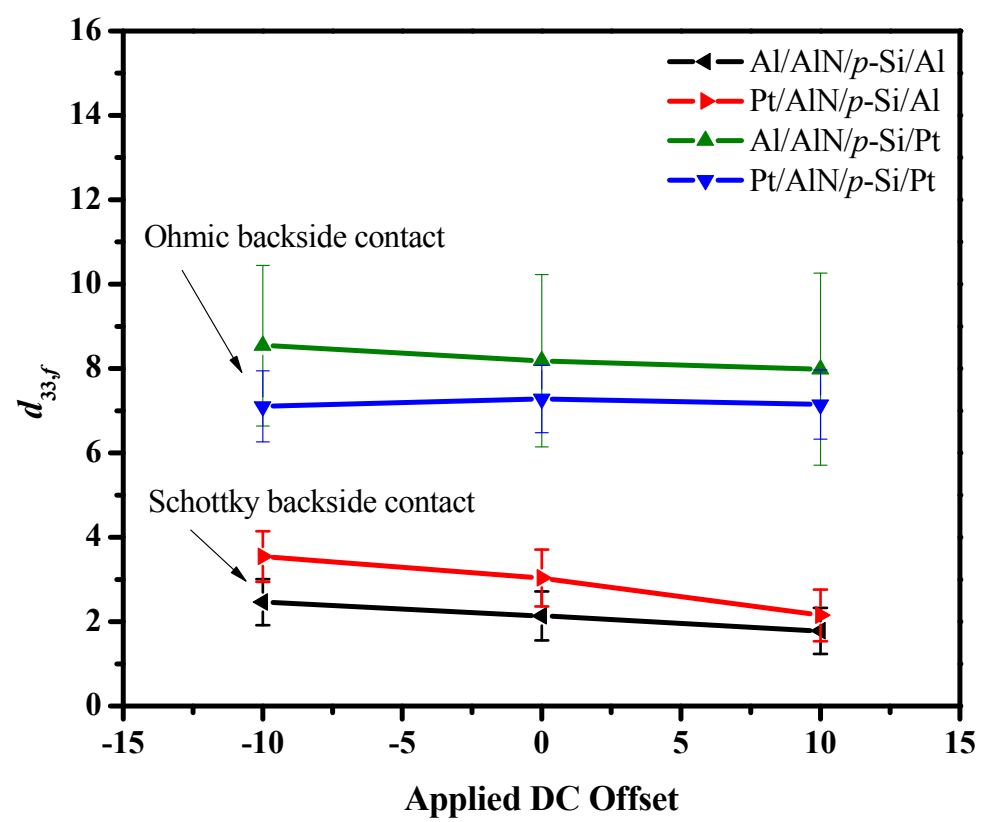

Figure 3.56. Measured effective longitudinal piezoelectric coefficient as a function of applied dc offset for four types of MIS structures.

\subsubsection{SUMMARY}

The contact metals used to fabricate AlN MIS structures clearly affects their effective piezoelectric properties. It is believed that this is due to an increase or a decrease in the internal electric field in the AlN layer. Recently, Zhao et al. reported the development of heterogeneous light emitting diodes fabricated by growing AlN on $p-\mathrm{Si}$ substrates. Gold contacts were used which formed Schottky contacts to the AlN film. The structure was biased and the resulting wavelength of the emitted light was measured. These results allowed this group to construct band diagrams of the AlN layer which are shown in Figure 3.57(a)-(c). 


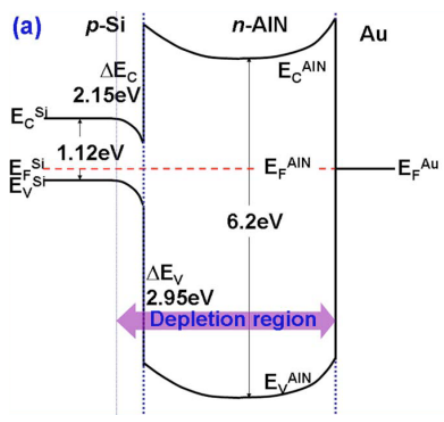

(a)

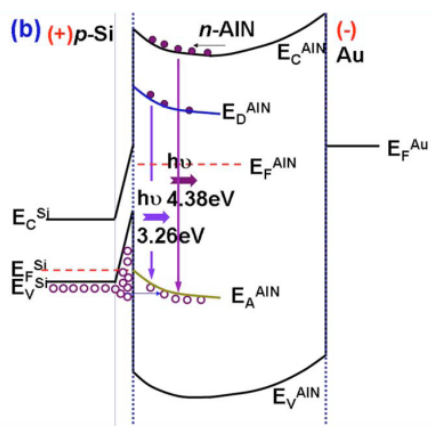

(b)

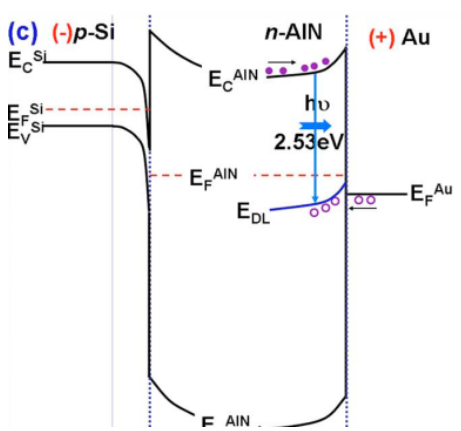

(c)

Figure 3.57. Band diagrams of heterogeneous LEDs fabricated by growing AlN on p-Si wafers. (a) zero applied bias, (b) negative applied bias, and (c) positive applied bias. The bias polarity was defined in terms of the Au/AlN interface [Zhao, 2009].

It is believe that the diagrams shown in Figure 3.57 support the idea that the built-in electric field can influence the effective piezoelectric response measured. 


\section{H A P T E R 4: TH I N F I L M P I E Z O E L E C T R I C D E V I C E S}

\subsection{BACKGROUND}

Thin piezoelectric films are commonly used in MicroElectroMechanical Systems (MEMS) devices. MEMS utilizing a piezoelectric active layer are commonly termed piezoelectric MEMS (pMEMS). MEMS translate an electrical stimulus into a mechanical response, or vice versa [Walraven, 2003]. These small devices offer several advantages over their bulk counterparts including reduced power consumption, reduced weight, lower cost, and in many cases, improved performance [Walraven, 2003]. In addition, MEMS devices can be fabricated using traditional semiconductor manufacturing techniques [Carrillo, 2003] making hybrid integrated circuits/MEMS devices possible.

\subsubsection{DEFINITION OF PIEZOELECTRICITY}

Piezoelectricity is defined as the capability of a material to produce electrical charge through the application of mechanical stress, or vice versa. This electro-mechanical coupling occurs in many crystal classes which lack an inversion center [Trolier-McKinstry, 2004]. There are two piezoelectric effects. In the direct effect, a force is applied to the piezoelectric material and a voltage is developed across it. In the converse effect, a potential is applied across the piezoelectric material leading to a structural deformation. Generally, the direct effect is used for sensing while the converse effect is used for actuation. Mathematically, the direct effect can be expressed as (4.1) where $D_{i}, d_{i k}$, and $\sigma_{i}$ are the components of the electric displacement field vector, piezoelectric tensor, and stress tensor, respectively [Trolier-McKinstry, 2004].

$$
D_{i}=\sum_{k=1}^{6} d_{i k} \sigma_{k}
$$

Likewise, the converse effect can be expressed as (4.2) where $x_{i}, d_{k i}$, and $E_{k}$ are components of the strain tensor, piezoelectric tensor, and electric field vector, respectively [Trolier-McKinstry, 2004]. 


$$
x_{i}=\sum_{k=1}^{3} d_{k i} E_{k}
$$

In wurtzite III-nitride crystals, the piezoelectric effect can be expressed in terms of the three independent piezoelectric strain coefficients $d_{33}, d_{31}$, and $d_{15}$. Due to symmetry in the $6 \mathrm{~mm}$ crystal class, $d_{31}=d_{32}$ and $d_{15}=d_{24}$ [Tonisch, 2006]. Additionally, in wurtzite crystals the $d_{31}$ component can be estimated if the $d_{33}$ component is known using the approximation given in (4.3) [Guy, 1999].

$$
d_{31} \approx-\frac{1}{2} d_{33}
$$

Thin films are deposited or grown on a substrate material. This typically results in a clamping of the film to the substrate. In this work, the clamped film piezoelectric strain coefficients, $d_{i j, f}$, are typically used because they are easy calculated using data provided by laser Doppler vibrometry measurements. The clamped strain coefficient, $d_{33, f}$, can be related to the transverse piezoelectric coefficient, $e_{33}$, and stiffness coefficient, $c_{33}$, using (4.4) [Dubois, 1999].

$$
d_{33, f}=e_{33} / c_{33}
$$

In addition, the clamped film piezoelectric transverse coefficient, $e_{31, f}$, can be related to the unclamped piezoelectric strain coefficient, $d_{31}$, and compliance coefficients, $s_{11}$ and $s_{12}$, using (4.5) [Dubois, 1999].

$$
e_{31, f}=\frac{d_{31}}{s_{11}+s_{12}}
$$

However, in order to relate the clamped and unclamped coefficients, the stiffness and/or compliance coefficients must be known [Dubois, 2001]. 


\subsubsection{Piezoelectric Materials}

Various piezoelectric materials are available for use in fabricating piezoelectric film based devices. The most common materials are Zinc Oxide ( $\mathrm{ZnO})$, Lead Zirconate Titanate (PZT), and Aluminum Nitride (AIN) [Sharma, 2003]. Table 4.1 lists the effective transverse piezoelectric coefficient, effective piezoelectric coefficient, and the dielectric constant for ZnO, AlN and PZT [Trolier-McKinstry, 2004]. The subscript $f$ indicates that the film is clamped to a rigid substrate [Dubois, 2001].

Table 4.1. Coefficients of various piezoelectrics. After Trolier-McKinstry and Muralt [Trolier-McKinstry, 2004].

\begin{tabular}{l|l|l|l}
\hline Coefficient & ZnO & AlN & PZT (1-3 $\mathbf{~ m m})$ \\
\hline$e_{31, f}\left(\mathrm{Cm}^{-2}\right)$ & -1.0 & -1.05 & $-8 \ldots-12$ \\
\hline$d_{33, f}(\mathrm{pm} / \mathrm{V})$ & 5.9 & 3.9 & $60 \ldots .130$ \\
\hline$\varepsilon_{33}$ & 10.9 & 10.5 & $300 . .1300$ \\
\hline
\end{tabular}

\subsubsection{Constituent Equations}

The stress, $\sigma$, and strain, $\varepsilon$, in a material can be related using Hooke's Law (4.6) where $E$ is Young's modulus.

$$
\sigma=E \varepsilon
$$

This relationship is valid over a region of small strain values (i.e. up to the material's proportional limit). This is shown graphically in Figure 4.1 for a brittle material such as silicon and a ductile material such as mild steel [Campbell, 2001]. 


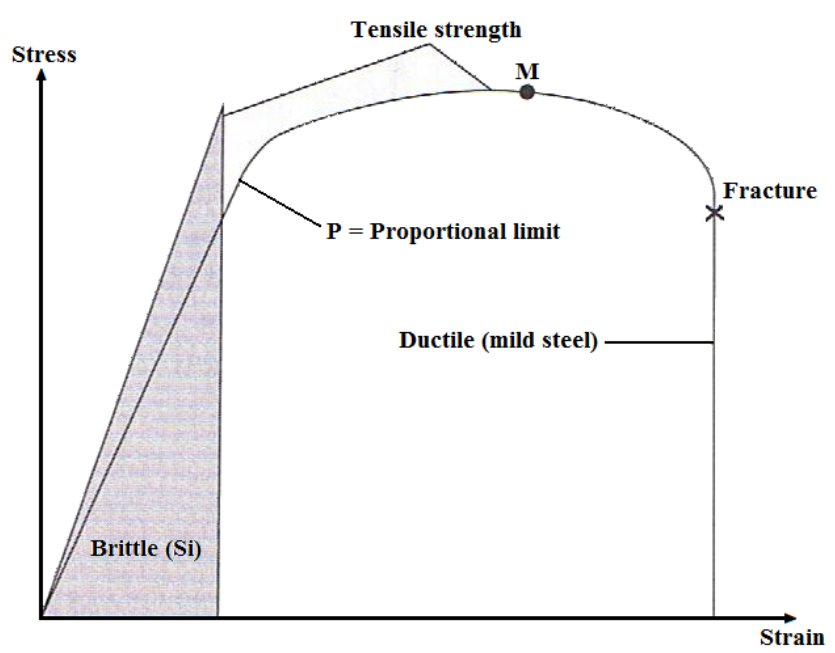

Figure 4.1. Stress-strain curves for a brittle and ductile material [Campbell, 2001].

Piezoelectric materials also have a contribution stemming from their electromechanical coupling. The constituent equations for a piezoelectric material account for this coupling along with describing its stress-strain relationship. The constituent equations of a piezoelectric material can take on four similar forms and are provided in Table 4.2 [Jaffe, 1965; Jaffe, 1971].

Table 4.2. Constituent equations for a piezoelectric material. After Jaffe [Jaffe, 1965; Jaffe, 1971].

\begin{tabular}{c|c|c|c}
\hline $\mathbf{( 1 )}$ & $\mathbf{( 2 )}$ & $\mathbf{( 3 )}$ & $\mathbf{( 4 )}$ \\
\hline$D=\varepsilon^{T} E+d T$ & $D=\varepsilon^{S} E+e S$ & $E=\beta^{T} E-g T$ & $E=\beta^{S} D-h S$ \\
$S=d_{t} E+s^{E} T$ & $T=-e_{t} E+c^{E} S$ & $S=g_{t} D+s^{D} T$ & $T=-h_{t} D+c^{D} S$ \\
\hline
\end{tabular}

Generally, the first set of equations is preferred. The superscript $T$ indicates that the quantity is measured at constant (zero) stress while the superscript $S$ indicates that the quantity is measured at constant (zero) strain. Quantities that are measured at constant strain are sometimes also referred to as "clamped" [Jaffe, 1971]. Table 4.3 provides a useful reference for relating the symbols in constituent equations to their respective quantities. 
Table 4.3. Symbols and units for electroelastic constants. After Jaffe [Jaffe, 1965].

\begin{tabular}{l|l|l}
\hline & \multicolumn{1}{|c|}{ Symbol } & \multicolumn{1}{c}{ Unit } \\
\hline Stress & $T$ & $\mathrm{~N} / \mathrm{m}^{2}$ \\
Strain & $S$ & $\mathrm{~m} / \mathrm{m}$ \\
Electric field strength & $E$ & $\mathrm{~V} / \mathrm{m}$ \\
Electric displacement & $D$ & $\mathrm{C} / \mathrm{m}^{2}$ \\
\hline & & \\
Elastic compliance & $S$ & $\mathrm{~m} / \mathrm{N}$ \\
Elastic stiffness & $C$ & $\mathrm{~N} / \mathrm{m}^{2}$ \\
Permittivity & $E$ & $\mathrm{~m} / \mathrm{F}$ \\
Dielectric impermeability & $B$ & $\mathrm{C} / \mathrm{N}$ or m/V \\
Piezoelectric strain constant & $D$ & $\mathrm{C} / \mathrm{m}^{2}$ or N$/ \mathrm{Vm}$ \\
Piezoelectric stress constant & $E$ & $\mathrm{Vm} / \mathrm{N}$ or $\mathrm{m}^{2} / \mathrm{C}$ \\
Piezoelectric strain constant & $G$ & $\mathrm{~V} / \mathrm{m}^{2} \mathrm{~N} / \mathrm{C}$ \\
Piezoelectric stress constant & $H$ & \\
\hline & & \\
Piezoelectric coupling factor & $K$ & \\
Mechanical quality factor & $Q_{M}$ & \\
Electrical quality factor & $Q_{E}=1 / \tan \delta$ & $\mathrm{m} / \mathrm{s}$ \\
Elastic velocity & $V$ & $\mathrm{~kg} / \mathrm{m}^{3}$ \\
Density & $P$ & \\
\hline
\end{tabular}

The electroelastic tensor for the $6 \mathrm{~mm}$ crystal class (of which AlN is a member of) contains the nonzero constants given in (4.7)-(4.9).

$$
\begin{aligned}
& s_{11}=s_{22}, s_{33}, s_{12}, s_{13}=s_{23}, s_{44}=s_{55}, s_{66}=2\left(s_{11}-s_{12}\right) \\
& d_{31}=d_{32}, d_{15}=d_{24}, d_{33} \\
& \varepsilon_{11}=\varepsilon_{12}, \varepsilon_{33}
\end{aligned}
$$

An electroelastic matrix is a convenient construct for expressing the dielectric, piezoelectric, and compliance coefficients of a specific crystal class. The electroelastic matrix for the $6 \mathrm{~mm}$ crystal class is shown given in Table 4.4 . 
Table 4.4. Electroelastic matrix for the 6mm crystal class. After Jaffe [Jaffe, 1965; Jaffe, 1971].



\subsubsection{ENHANCED PieZOELECTRIC EFFECT}

An enhanced piezoelectric effect is desirable for developing sensors and actuators with improved performance. We have shown that the effective piezoelectric strain coefficient, $d_{33, f}$, of AlN thin films can be enhanced by depositing high work function metal contacts on the topside of AlN films [Harman, 2008]. We have also shown that thin AlN films reactively sputtered using an $\mathrm{Al}_{0.97} \mathrm{Er}_{0.03}$ target have higher effective piezoelectric strain coefficients when compared to AlN films reactively sputtered using a pure $\mathrm{Al}$ target [Kabulski, 2009].

\subsubsection{Metal Contact Work Function}

The effective piezoelectric strain coefficient of AlN thin films was found to be a function of the metal topside contact (see Figure 3.6). Higher work function metals such as platinum resulted in higher measured displacements while lower work function metals such as aluminum resulted in lower measured displacements (see Figure 3.7). Additionally, the piezoelectric response of sputtered AlN thin films was found to be a function of the film thickness as shown in Figure 4.2 [Harman, 2008]. 




Figure 4.2. Effective piezoelectric strain coefficient of various AlN thin films. After Harman et al. [Harman, 2008].

This agrees with the data plotted for sputtered AlN films shown in Figure 2.13. We believe that this effect can be explained in terms of the quality of the films as a function of film thickness.

\subsubsection{RARE EARTH DOPING}

Rare earth (RE) metals have been used extensively to tune the properties of Lead Zirconate Titanate (PZT) thin films. PZT is a ferroelectric material having high piezoelectric coefficients. Small changes in the composition of PZT can lead to the material taking on forms from various ferroelectric phases to paraelectric cubic phases. The short-range forces in PZT favor the ferroelectric phases while the long-range Coulombic forces favor the paraelectric phases. Thus, REs are commonly used to modify the material properties of PZT such as its Curie temperature, conductivity, coercitivity, and compliance [Lappalainen, 1999]. 
Shannigrahi et al. performed a study to determine the effect of substituting various $\mathrm{RE}$ ions $(\mathrm{R}=$ Lanthanum (La), Neodymium $(\mathrm{Nd})$, Samarium $(\mathrm{Sm})$, Europium (Eu), Gadolinium (Gd), Dysprosium (Dy), Erbium (Er), and Ytterbium (Yb)) at the $\mathrm{Pb}$ site in $\mathrm{PZ}_{0.60} \mathrm{~T}_{0.40}$. PZT with a $\mathrm{Zr} / \mathrm{Ti}$ ratio of $60 / 40$ was chosen since this composition is close to the Morphological Phase Boundary (MPB). The effect of substituting $8 \%$ of the lead ions with RE ions on the crystal structure, electric, and piezoelectric properties was investigated. The results of this study are provided in Table 4.5 [Shannigrahi, 2004].

Table 4.5. Material properties of RE-doped PZT films. After Shannigrahi et al. [Shannigrahi,2004].

\begin{tabular}{l|l|l|l|l|l|l|l|l|l|l}
\hline $\mathbf{R E}$ & $\boldsymbol{\varepsilon}_{\mathbf{R T}}$ & $\begin{array}{l}\tan \\
\boldsymbol{\delta}_{\mathbf{R T}}\end{array}$ & $\boldsymbol{\varepsilon}_{\mathbf{m a x}}$ & $\begin{array}{l}\tan \\
\boldsymbol{\delta}_{\mathbf{m a x}}\end{array}$ & $\begin{array}{l}\boldsymbol{T}_{\mathbf{c}} \\
\left({ }^{\left.{ }^{\mathbf{C}}\right)}\right.\end{array}$ & $\boldsymbol{\gamma}$ & $\begin{array}{l}\boldsymbol{E}_{\mathbf{a}} \\
\mathbf{( e V )}\end{array}$ & $\begin{array}{l}\boldsymbol{P}_{\mathbf{r}} \\
\left(\boldsymbol{\mu C} / \mathbf{c m}^{2}\right)\end{array}$ & $\begin{array}{l}\boldsymbol{E}_{\mathbf{c}} \\
(\mathbf{k V / c m})\end{array}$ & $\begin{array}{l}\boldsymbol{d}_{\mathbf{3 3}} \\
(\mathbf{p C} / \mathbf{N})\end{array}$ \\
\hline $\mathrm{La}$ & 3413 & 0.072 & 18924 & 0.016 & 156 & 1.56 & 0.53 & 21.90 & 6.65 & 569 \\
\hline $\mathrm{Nd}$ & 2148 & 0.005 & 6577 & 0.007 & 182 & 1.68 & 0.25 & 8.84 & 6.61 & 269 \\
\hline $\mathrm{Sm}$ & 737 & 0.071 & 5594 & 0.051 & 284 & 1.70 & 0.18 & 7.58 & 5.51 & 151 \\
\hline $\mathrm{Eu}$ & 404 & 0.080 & 7588 & 0.070 & 349 & 1.92 & 0.26 & 5.41 & 5.28 & 53 \\
\hline $\mathrm{Gd}$ & 187 & 0.098 & 12864 & 0.064 & 337 & 1.91 & 0.63 & 8.31 & 3.11 & - \\
\hline $\mathrm{Dy}$ & 528 & 0.085 & 12673 & 0.014 & 368 & 1.48 & 0.33 & 6.75 & 3.97 & 84 \\
\hline $\mathrm{Er}$ & 422 & 0.046 & 11123 & 0.031 & 354 & 1.40 & 0.86 & 10.78 & 7.22 & 75 \\
\hline $\mathrm{Yb}$ & 194 & 0.029 & - & 0.086 & - & - & - & - & - & - \\
\hline
\end{tabular}

Work has also been reported on the RE doping of AlN thin films. However, most efforts have focused on the optoelectronic properties of RE-doped films [Jadwisienczak, 2000; Dorenbos, 2006] and not on their piezoelectric properties. One major subset of research in the area of RE-doped AIN is the incorporation of erbium optical centers for use in silica fiber-based communication systems [MacKenzie, 1996]. Erbium ions $\left(\mathrm{Er}^{3+}\right)$ are known to have a host independent optical transition near $1.54 \mu \mathrm{m}$ which turns out to correspond to the wavelength of minimum attenuation loss in silica fibers. Thus, AlN is an ideal host material for erbium-based telecommunications devices because AIN is a wide bandgap material and because it has high temperature stability [MacKenzie, 1996; Wilson, 1994]. For example, erbium emission was found to be unaffected even when AlN samples were annealed at temperatures up to $900^{\circ} \mathrm{C}$ [Wilson, 1994]. 
Brien et al. performed a study to determine the PhotoLuminescence (PL) spectra of Er-doped AlN films over various erbium incorporations. They prepared the films using rf magnetron sputtering by placing small pieces of erbium on top of an aluminum target. Five films were successively prepared using identical sputtering parameters. The first film prepared had the highest erbium content while the last film had the smallest. This was due to a decrease in the amount of erbium remaining on the aluminum target. Energy dispersive X-ray spectroscopy was used to determine the concentration of erbium incorporated in the films. The atomic percent erbium along with the PL spectra for the five films are shown in Figure 4.3 [Brien, 2007].

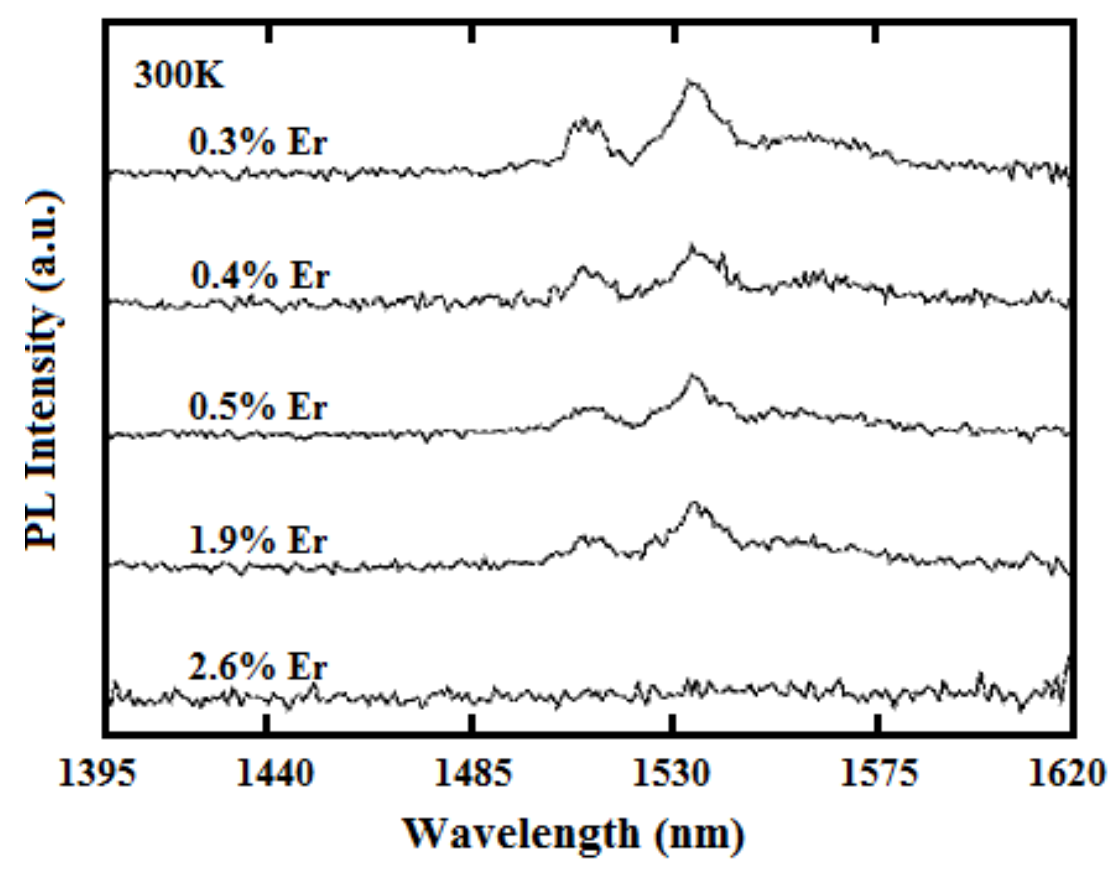

Figure 4.3. Photoluminescence spectra of Er-doped reactively sputtered AlN films. After Brien et al. [Brien, 2007].

It can be noted that the maximum $\mathrm{Er}^{3+}$ emission at $1.54 \mu \mathrm{m}$ occurs at an erbium atomic concentration of $0.3 \%$. Thus, it can be inferred that the highest PL intensity occurs for erbium concentrations between $0 \%$ and $0.3 \%$. The diminishing PL intensity for higher erbium concentrations was suggested to be due to concentration quenching [Brien, 2007]. 
Of particular interest is the physical location of erbium ions when incorporated in reactively sputtered AIN thin films. As shown in Figure 4.4, the erbium ions could substitute aluminum ions or nitrogen ions (C) or they could form interstitials (B). Additionally, they could react with oxygen atoms present during the deposition of AIN and form erbium oxide.

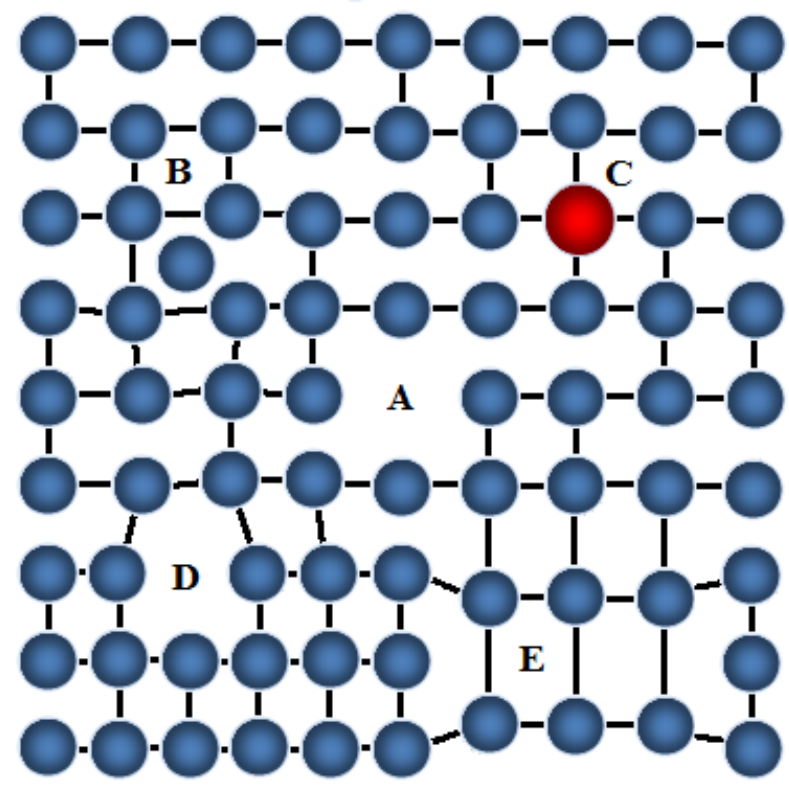

Figure 4.4. Various types of defects occurring in a crystal. (A) vacancies, (B) selfinterstitials, (C) substitutional impurities, (D) edge dislocations, and (E) dislocation loops. After Campbell [Campbell, 2001].

We performed XPS on Er-doped AIN thin films to try to find the chemical shift of the erbium peak (see Section 3.2.3.5). However, the amount of erbium incorporated was determined to be below the XPS detection limit of 0.1 atomic percent. This agrees with the small amount of erbium incorporated in the study conducted by Brien et al. [Brien, 2007] Additional methods for determining the RE concentration in Er-doped AIN films include photoluminescence [Wilson, 1994; MacKenzie, 1996], Secondary Ion Mass Spectrometry (SIMS) [Wilson, 1994], and CathodoLuminescence (CL) [Jadwisienczak, 2000]. 


\subsection{MEMS}

MicroElectroMechanical Systems (MEMS) are microscopic devices that interact with their environment. They translate a mechanical or electrical stimulus into a mechanical or electrical response. For example, devices can be fabricated that translate acceleration (mechanical stimulus) into corresponding electrical signals (electrical response). Many types of MEMS are commercially available. Examples are sensors, switches, tunable capacitors, antennas, transmission lines, filters and resonators [Allen, 2004].

MEMS devices offer several advantages over their bulk counterparts which include reduced power consumption, reduced weight, lower cost and in some cases improved performance [Walraven, 2003] In addition, MEMS devices can be fabricated using traditional semiconductor manufacturing techniques [Carrillo, 2003]. This means that current micromachining techniques including photolithography, etching, and thin film deposition techniques used to fabricate modern Integrated Circuits (ICs) can be used to fabricate silicon based MEMS devices [Baborowski, 2004]. Finally, since current semiconductor technologies are silicon based, hybrid devices integrating ICs and MEMS can be fabricated on a single substrate.

\subsection{1 pMEMS}

pMEMS typically consist of a bulk silicon frame and a deflection element with electrodes [Baborowski, 2004]. Using a piezoelectric material in a MEMS device offers several advantages including a high frequency constant, good temperature stability, low power requirements, mild hysteresis, high energy density when scaled, and a complete compatibility with Complementary Metal Oxide Semiconductor (CMOS) devices [TrolierMcKinstry, 2004]. Piezoelectric films also allow the both sensing and actuation functions to be implemented on a single MEMS device [Xu, 2002]. Finally, the planar structure of piezoelectric films produces an out-of-plane film deflection which is useful for many sensor applications [Baborowski, 2004]. 


\subsubsection{Piezoelectric Materials}

For pMEMS devices, the two most important criteria for the piezoelectric material are a high piezoelectric response and a controlled residual stress [Iborra, 2004]. Additionally, a low dielectric constant generally is desired. The signal-to-noise (SNR) ratio for ac signals (when the main source of noise is due to the piezoelectric) can be related to the piezoelectric coefficient, $e_{31, f}$, the dielectric constant, $\varepsilon_{33, f}$, and the loss tangent, $\tan \delta$, as expressed in (4.10) [Muralt, 1997].

$$
S N R \propto \frac{e_{31, f}}{\sqrt{\varepsilon_{33, f} \cdot \tan \delta}}
$$

By examining the data in Table 4.1, the piezoelectric coefficient and dielectric constant of PZT can be seen to be much higher than that of AIN and ZnO. This makes PZT the more favorable choice for charge detection and either $\mathrm{ZnO}$ or AlN the more favorable choice for voltage detection. The SNR is approximately the same for PZT, ZnO, and AlN [Muralt, 1997].

The force output of a piezoelectric is proportional to its piezoelectric coefficient and the power output of a piezoelectric is proportional to the square of its piezoelectric coefficient. Additionally, the power output is proportional the piezoelectric thickness [Duval, 2006]. Thus, thick PZT films are best when maximum force or power output is desired. The power efficiency, $k^{2}$, is proportional to the square of the piezoelectric coefficient, $e_{31, f}$, divided by the dielectric constant, $\varepsilon_{33, f}$, as given in (4.11) [Muralt, 1997].

$$
k^{2} \propto \frac{\left(e_{31, f}\right)^{2}}{\varepsilon_{33, f}}
$$

This relation shows that the power efficiency of PZT may not be the best (see table 4.1). Additionally, the dielectric losses in PZT are typically higher than those of $\mathrm{ZnO}$ and AlN. Thus, AlN and $\mathrm{ZnO}$ are competitive in applications where the highest efficiency is 
desired [Muralt, 1997]. Most piezoelectric devices utilize PZT thin films [Baborowski, 2004; Trolier-Mckinstry, 2004; Muralt, 1997; Chapuis, 2006]. PZT is a ferroelectric material with large piezoelectric coefficients [Xu, 2002; Trolier-Mckinstry, 2004]. The major disadvantages of PZT films are 1) an incompatibility between PZT films and CMOS technology [Sharma, 2003] and 2) a high structural dependence on temperature [TrolierMckinstry, 2004]. ZnO films are also used as the deflection element in pMEMS. ZnO is a piezoelectric material with piezoelectric coefficients similar to AIN [Trolier-Mckinstry, 2004]. The disadvantages of using $\mathrm{ZnO}$ include 1) an incompatibility between $\mathrm{ZnO}$ and current silicon semiconductor technology [Trolier-Mckinstry, 2004], 2) an incompatibility between $\mathrm{ZnO}$ and CMOS technology [Sharma, 2003] and 3) a lower film resistivity when compared to AlN [Trolier-Mckinstry, 2004].

\subsubsection{Piezoelectric Structures}

Common piezoelectric structures include diaphragms, microbridges, and cantilevers. Examples are shown in Figure 4.5. These structures can be fabricated using standard micromachining technology. They are regularly used separately in pMEMS. In addition, they can be combined to form more complicated pMEMS devices.

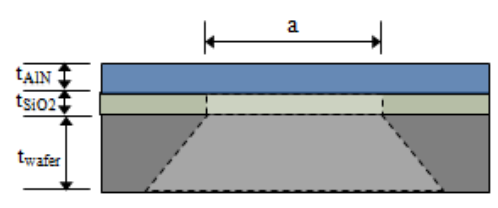

Side View



Top View

Diaphragm

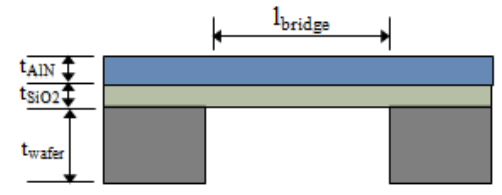

Side View

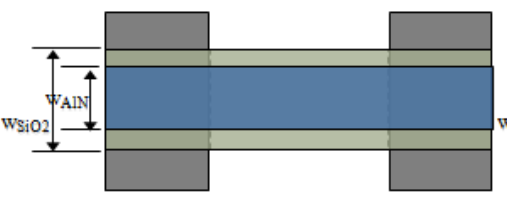

Top View

Microbridge

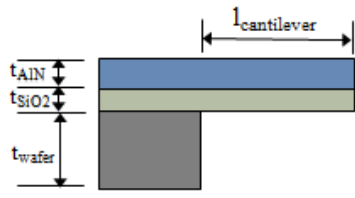

Side View

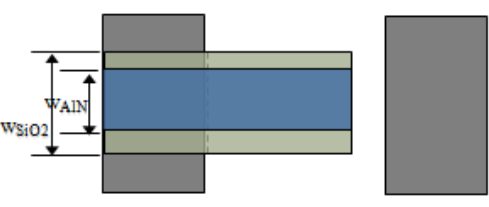

Top View

Cantilever

Figure 4.5. Common piezoelectric structures. 
In this work, diaphragm structures were fabricated using bulk micromachining. Additionally, cantilever structures were simulated in 2- $d$ and 3- $d$ using finite element analysis.

\subsubsection{Electrode Configurations}

In addition to the geometry of the piezoelectric structure, the configuration of the electrode is important to the operation of the pMEMS device. Two common electrode configurations are the planar capacitor and the InterDigitated Transducer (IDT) and are shown in Figure 4.6 [Trolier-McKinstry, 2004].

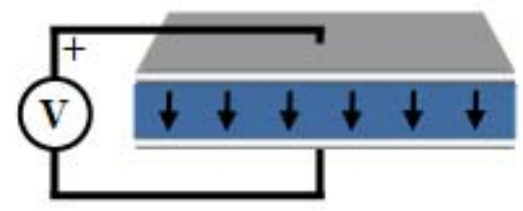

(a)



(b)

Figure 4.6. Common electrode configurations. (a) planar capacitor and (b) interdigitated transducer. After Trolier-McKinstry and Muralt [Trolier-McKinstry, 2004].

\subsubsection{DIAPHRAGMS}

Diaphragm structures are commonly used in microvalves [Koch, 1996] and Thin Film Bulk Acoustic Resonators (TFBARs) [Dubois, 1998], among others. Additionally, they can be used as the base for Lamb wave mode surface acoustic wave devices (discussed in Section 5.1.2). An example of a microvalve system utilizing bulk micromachined diaphragms is shown in Figure 4.7 [Koch, 1996]. The cantilevers served as passive one-way valves to control the flow of fluid through the diaphragm apertures [Koch, 1996]. 


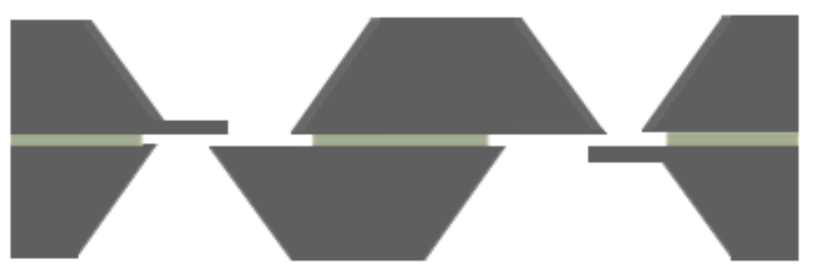

$\mathrm{SiO}_{2}$

Si Substrate

Figure 4.7. Cantilever and diaphragm based microvalve system. After Koch et al. [Koch, 1996].

Diaphragms can be fabricated from silicon wafers using bulk micromaching techniques. Wet etching using a preferential silicon etchant is a common method for producing pyramidal shaped cavities in (100) silicon wafers. Transene PSE-300F ethylene diamine etchant preferentially attacks the (100) planes leaving exposed (111) sidewalls as shown in Figure 4.8.



Figure 4.8. Preferential etching of (100) silicon wafers with PSE-300F.

In order to design diaphragm based devices, it is important to be able to control the dimensions of the diaphragms for a particular application. The cosine of the angle between planes in a silicon wafer can be determined using (4.12).

$$
\cos (\theta)=\frac{h_{1} h_{2}+k_{1} k_{2}+l_{1} l_{2}}{\left(h_{1}^{2}+k_{1}^{2}+l_{1}^{2}\right)^{1 / 2}\left(h_{2}^{2}+k_{2}^{2}+l_{2}^{2}\right)^{1 / 2}}
$$


The angle, $\theta$, between the (100) planes and the (111) planes can be found by rearranging (4.12) and substituting $h_{1}=1, k_{1}=0, l_{1}=0, h_{2}=1, k_{2}=1, l_{2}=1$ as shown in (4.13).

$$
\theta=\cos ^{-1}\left(\frac{1}{\sqrt{3}}\right) \cong 54.74^{\circ}
$$

By determining the angle $\theta$ and by knowing the thickness of the silicon wafer, $t_{\text {wafer }}$,

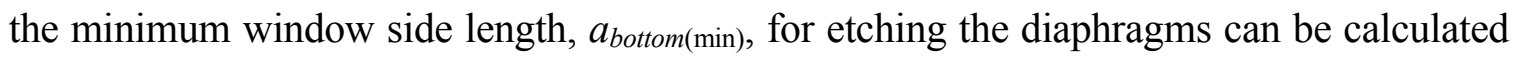
using (4.14).

$$
a_{\text {bottom(min) }}=\frac{2 t_{\text {wafer }}}{\tan (\theta)}
$$

In this work, double-side polished silicon wafers with a thickness between $300 \mu \mathrm{m}$ and $350 \mu \mathrm{m}$ were used. Using the worst case scenario (i.e. $t_{\text {wafer }}=350 \mu \mathrm{m}$ ), the minimum window side length was determined to be approximately $500 \mu \mathrm{m}$.

$$
a_{\text {bottom }(\min )}=\frac{2(350 \mu m)}{\tan \left(54.74^{\circ}\right)}=494.89 \mu m
$$

After this minimum window side length, the diaphragm side length is proportional to the window side length. An approximation for the diaphragm side length neglecting the contribution due to etching of the (111) planes is given by (4.16).

$$
a_{\text {diaphragm }} \cong a_{\text {window }}-a_{\text {bottom(min) }}
$$

The diaphragms were fabricated by coating the double sided silicon wafers with silicon dioxide. The silicon dioxide films were deposited using reactive e-beam 
evaporation or Plasma Enhanced Chemical Vapor Deposition (PECVD). After coating both sides with silicon dioxide, HexaMethylDiSilazane (HMDS) and AZ5214-E image reversal (IR) photoresist (PR) were spun on both sides. The PR was exposed on one side of the wafer (termed the "backside") through a photomask and the other side (termed the "topside") was exposed without a mask using a flood exposure system. The wafer was baked at $120^{\circ} \mathrm{C}$ for 2 minutes in order to crosslink the PR and both sides were exposed using a flood exposure system for 82 seconds. The PR was developed in a 1:1 solution of MIF-312 developer to deionized water. Next, the silicon dioxide exposed by the windows in the photoresist was etched using J.T. Baker Buffered Oxide Etchant (BOE). The wafer was rinsed in cascading deionized water for 10 minutes and the photoresist was removed using acetone and methanol. Finally, the silicon wafer was etched using Transene PSE$300 \mathrm{~F}$ preferential silicon etchant. It was experimentally found that the etch rate of PSE$300 \mathrm{~F}$ is highly dependent on the temperature of the etchant. Thus, the wafer was removed from the etchant periodically to determine the etching progress. Silicon dioxide diaphragms fabricated using a circular mask are shown in Figure 4.8. Notice that although the window is circular, the resulting diaphragm is square due to the anisotropic nature of the etchant.

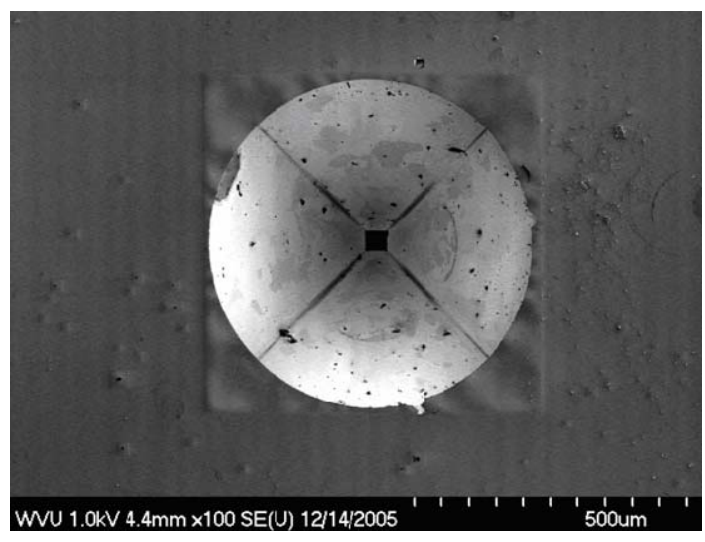

(a)

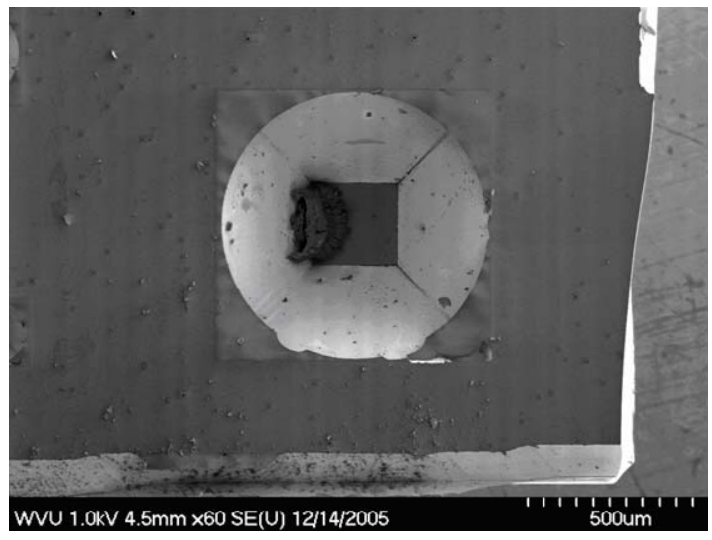

(b)

Figure 4.8. SEM images of diaphragms fabricated with a circular mask. (a) backside, $a_{\text {diaphragm }} \approx 43 \mu \mathrm{m}$ and (b) backside, $a_{\text {diaphragm }} \approx 230 \mu \mathrm{m}$. 
A window mask (shown in Appendix B) with $a_{\text {bottom }}=730 \mu \mathrm{m}$ was designed and used to fabricate silicon dioxide diaphragms. Aluminum nitride was reactively sputtered on the silicon dioxide diaphragms to create $\mathrm{AlN} / \mathrm{SiO}_{2}$ diaphragms. The wafer was scribed through the diaphragm and the corresponding cross-sectional image is shown in Figure 4.9(a). An image of the backside of the wafer is also shown in Figure 4.9(b).

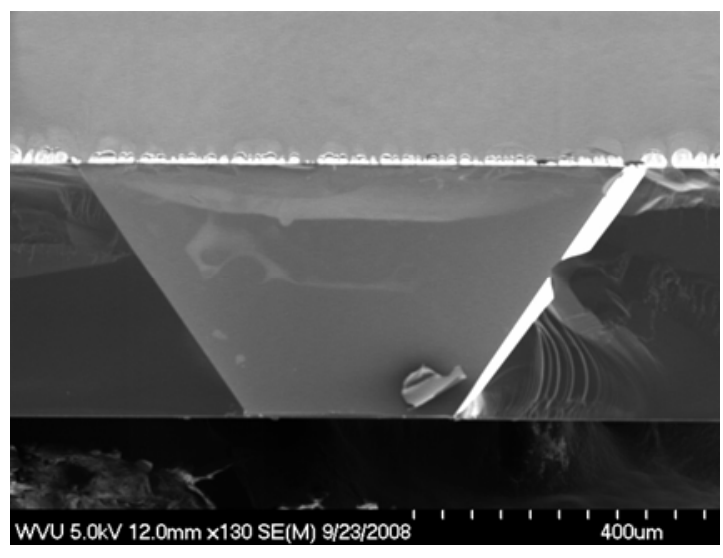

(a)

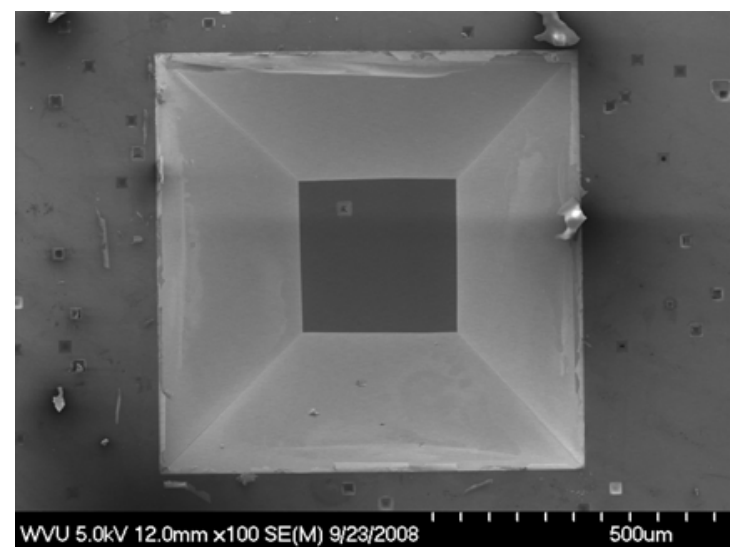

(b)

Figure 4.9. SEM images of diaphragms fabricated with the square mask shown in Appendix B. (a) cross-section and (b) backside.

If the wafer is not completely etched through to the silicon dioxide film, the silicon dioxide can be removed using hydrofluoric acid to leave pure silicon diaphragms. The thickness of the silicon diaphragms is not easily controllable due to the variance in wafer thickness $(300 \mu \mathrm{m}$ to $350 \mu \mathrm{m})$ and the temperature dependence of the etchant. However, aluminum nitride films deposited on pure silicon are typically of much higher quality than films deposited on silicon dioxide (see Section 2.2.2.3). Pure silicon diaphragms are shown in Figure 4.10. 


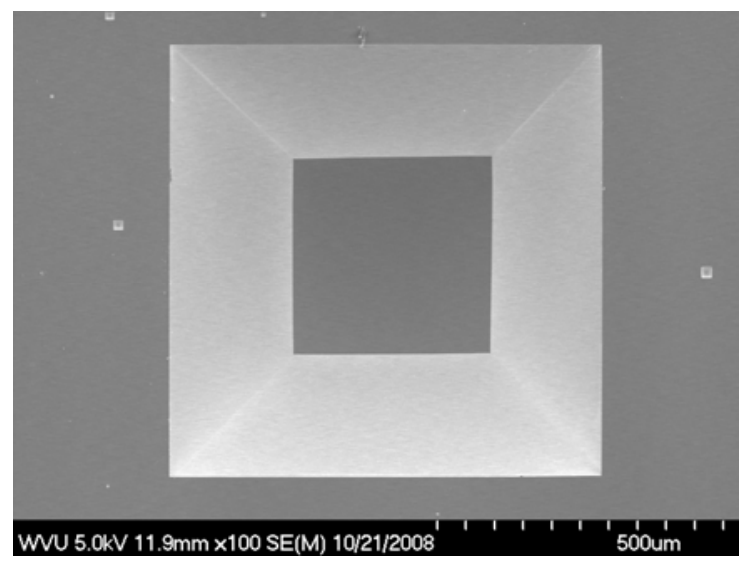

(a)

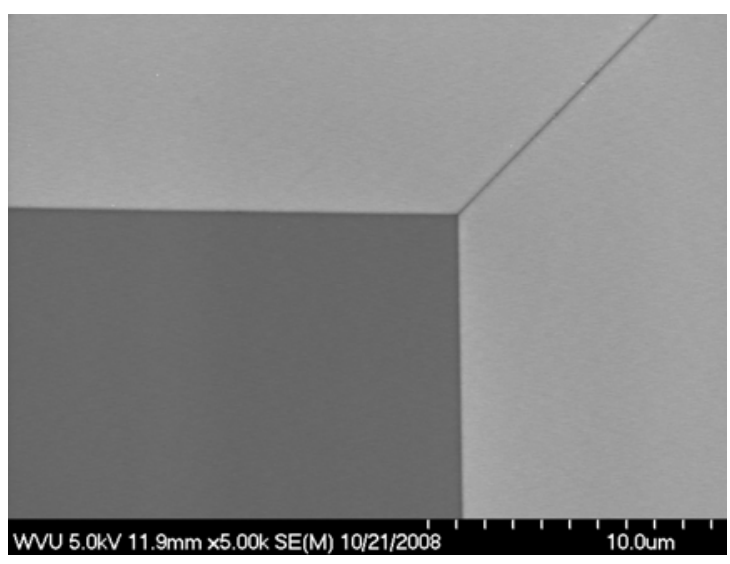

(b)

Figure 4.10. SEM images of pure silicon diaphragms fabricated with the square diaphragm mask (see Appendix B). (a) backside and (b) corner.

\subsubsection{PIEZOELECTRIC BEAMS}

Piezoelectric beams are commonly used in piezoelectric microelectromechanical systems (pMEMS) and have many possible applications in smart sensor and actuator systems. Piezoelectric beams are a key structure for many pMEMS applications (see 4.2.3) [Huang, 2004]. For example, they have been used as the active element in microfluidic and microactuator pMEMS devices. In the actuator mode, they employ the converse piezoelectric effect to couple electrical energy into mechanical deformation.

Various types of piezoelectric beams can be developed including unimorphs, bimorphs, and multimorphs as shown in Figure 4.11.

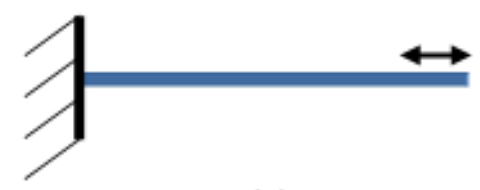

(a)

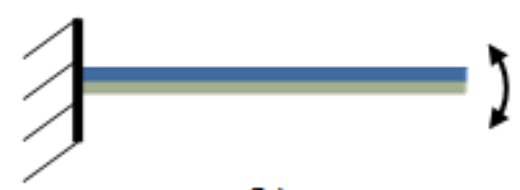

(b)

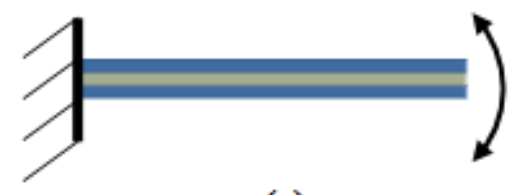

(c)

Piezoelectric

\section{Structural (non-piezoelectric)}

Figure 4.11. Various cantilever configurations. After Chapuis et al. [Chapuis, 2006]. 
For aluminum nitride based piezoelectric unimorphs (type a), the displacement is mainly in the plane perpendicular to the applied voltage. In bimorphs where one layer is structural and the other layer is piezoelectric, the cantilever displacement results in a bending motion. In a similar manner, for multimorphs consisting of two piezoelectric layers and a structural layer (type c), the displacement also results in a bending motion. The maximum tip displacement for the cantilever geometries in Figure 4.11 can be determined analytically. Chapuis et al. determined the maximum deflection of type $b$ piezoelectric bimorphs using (4.17) where $E_{b}, E_{p}, t_{b}$ and $t_{p}$ are Youngs' modulus and the thicknesses of the passive and piezoelectric materials, respectively, $V$ is the applied voltage, and $L$ is the total cantilever length [Chapuis, 2006].

$$
\delta_{\text {max }}=\frac{3 d_{31} t_{b} L^{2} V}{E_{b} t_{b}^{3} t_{p}\left(\frac{1}{E_{b} t_{b}}+\frac{3 t_{b}\left(t_{b}+t_{p}\right)}{E_{b} t_{b}^{3}}+\frac{1}{E_{p} t_{p}}\right)}
$$

Smits et al. derived expressions for determining the constituent equations of piezoelectric bimorphs [Smits, 1991]. The relationships were quite complicated and neglected internal stress that may be present in the films. Thus, finite element analysis is commonly performed in order to accurately determine the deflection of complicated piezoelectric MEMS structures [Chapuis, 2006; Olivares, 2005].

The multimorphs (type c) can take on three sub-types; namely, serial, parallel, and bipolar parallel. Finite element analysis was used to determine the affect of including the structural layer. The results of FEA indicated that the structural layer only acted to reduce the maximum deflection (not shown). For simplicity, the structural layer is not considered in this work and so the piezoelectric beams are termed "bimorphs". The serial bimorph is shown in Figure 4.12. In this configuration, two piezoelectric layers having opposite polarities are joined and a single external voltage source is applied across the structure. Since the structure is really a combination of two single unimorphs fused together, one of 
the unimorphs will contract along the direction perpendicular to the electric field and the other will expand. This produces a pure bending motion [Huang, 2004].

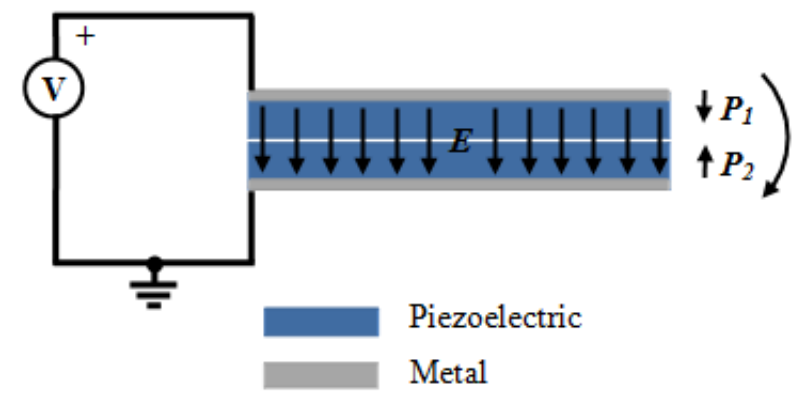

Figure 4.12. Serial bimorph. After Huang et al. [Huang, 2004].

The second multimorph sub-type is the parallel bimorph and is shown in Figure 4.13(a). In this configuration, two piezoelectric layers having the same polarity are joined together. The external voltage is applied across both of the layers by 1) grounding the top and bottom contacts and applying a voltage at the interface between the two layers or 2) by applying the voltage to the top and bottom contacts and grounding the interface between the two layers. In either case, an electric field with a magnitude double to that applied in the serial bimorph case is produced across each of the layers for a given applied voltage. Since the electric fields in the two layers have opposite directions and the layers have the same polarity, a pure bending motion is produced [Huang, 2004]. 


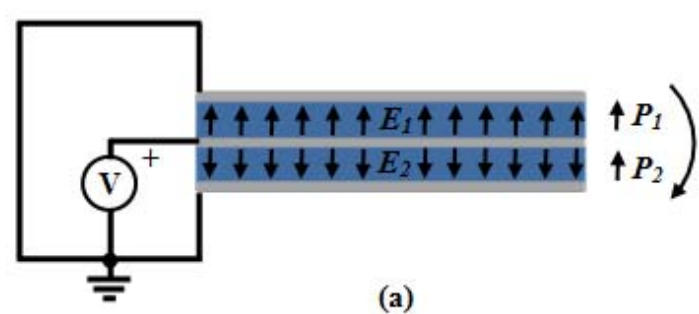

(a)

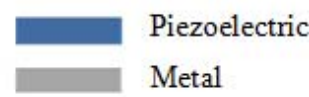

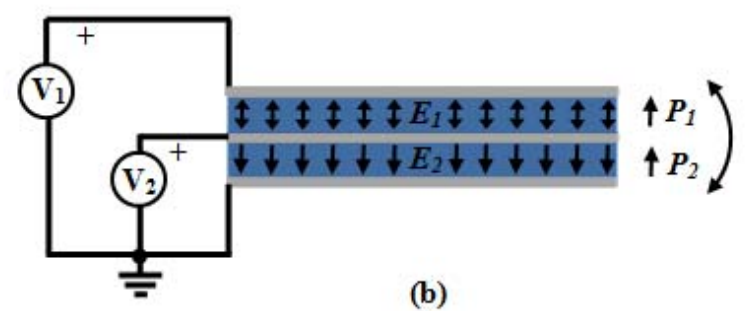

(b)

Figure 4.13. Parallel bimorphs. (a) parallel and (b) bipolar parallel. After Huang et al. [Huang, 2004].

The third multimorph sub-type is the bipolar parallel bimorph and is shown in Figure 4.13(b). In this configuration, two piezoelectric layers having the same polarity are joined. A separate external voltage is then applied across each of the layers. The bending motion can be controlled more accurately in this configuration. However, the tip deflection may not be as much as in the parallel bimorph case [Huang, 2004].

The geometry and position of the contacts are design parameters that have not been addressed much in the literature. Q. Q. Zhang et al. reported on the fabrication and simulation of $d_{33}$ cantilever actuators that used interdigitated contacts to produce electric fields in the plane of the piezoelectric layer [Zhang, 2003] while O. J. Aldraihem et al. derived analytical solutions for cantilever beams with piezoelectric patches [Aldraihem, 2003]. However, generally it is assumed that the top and bottom contacts span the entire length and width of the bimorph structure although it is known that the size of the topcontact affects the tip displacement [Prume, 2004]. This configuration undoubtedly produces the highest tip displacement in $d_{31}$ mode bimorphs and leads to the simplest analytical solutions. Nonetheless, the displacement distribution produced is approximately parabolic, and as such, may not be optimal for certain applications.

Bulk micromaching techniques were used to create free standing silicon dioxide diaphragms with dimensions $l=w \approx 290 \mu \mathrm{m}$ and $h \approx 400 \mathrm{~nm}$ on a silicon substrate (see Section 4.2.3.2). Aluminum nitride was deposited on the top surface of the diaphragm 
structure using DC magnetron reactive sputtering to form $\mathrm{AlN} / \mathrm{SiO}_{2}$ diaphragms. AlN can also be deposited on the bottom surface of the diaphragm structure using the same process. The top and bottom AlN film will then have opposing polarizations effectively creating a bimorph structure around an elastic layer. Simulations indicate that the presence of the elastic layer reduces the deflection of the bimorph (not shown). Thus, reducing the thickness of the elastic layer will improve the performance of the structure. The cantilevers can be released by etching patterned diaphragms using Inductively Coupled Plasma (ICP) Reactive Ion Etching (RIE) or by using laser ablation as shown in Figure 4.14. The resulting diaphragm-based bimorph cantilever structure is shown in Figure 4.15.

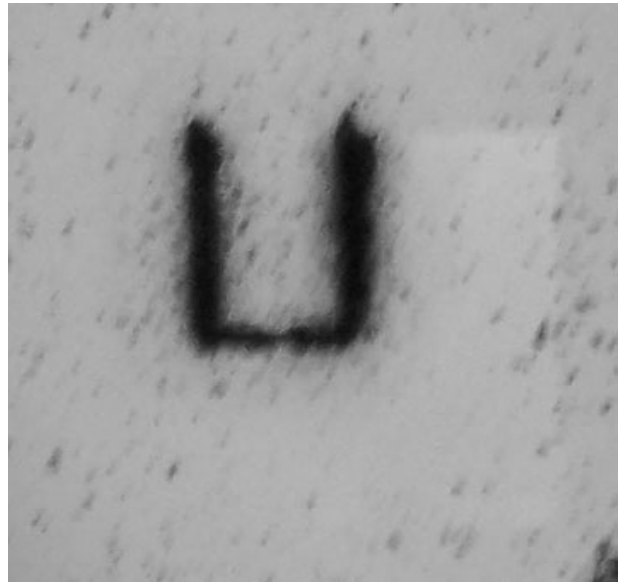

(a)

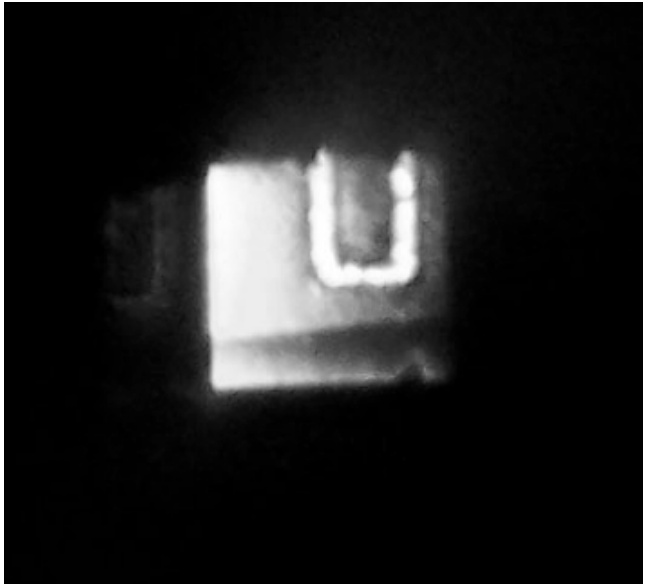

(b)

Figure 4.14. Optical microscope images of a laser ablated, diaphragm based cantilever. (a) topside image before solvent bath and (b) backside image after solvent bath. 


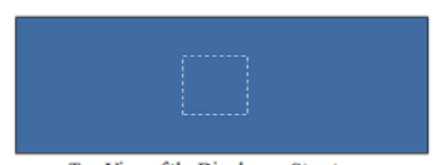

Top View of the Diaphragm Structure

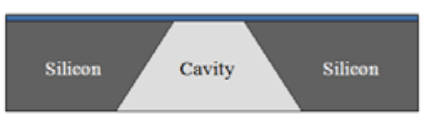

Cross Section of the Diaphragm Structure

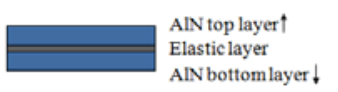

(a)

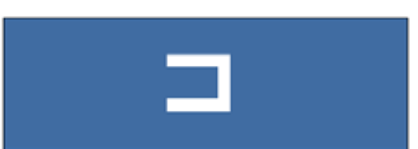

Top View of the Cantilever Structure

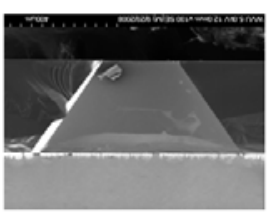

SEM Image of the Cross Section of the Diaphragm Structure



Cross Section of the Cantilever Structure

AIN top layer $(0.5 \mu \mathrm{m}) t^{t}$ Elastic layer $(0 \mu \mathrm{m})$ AN bottom layer $(0.5 \mu \mathrm{m})$

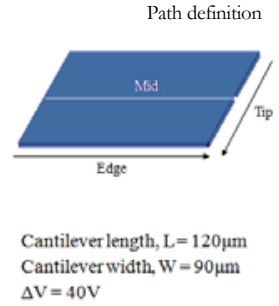

(b)

Figure 4.15. (a) Bulk micromachined diaphragm structure and (b) cantilever structure simulated using finite element analysis.

The deflection of a cantilever with $l>>w$ is given by (4.18) where $f$ is a uniformly distributed load, $E$ is Young's modulus, $I=(1 / 12) w h^{3}$ is the moment of inertia, and $l$ is the cantilever length.

$$
\delta(y)=\frac{f y^{2}}{24 E I l}\left(y^{2}-4 l y+6 l^{2}\right)
$$

For a given cantilever, the dimensions and material properties are fixed. Additionally, the resulting distribution is typically parabolic. The maximum deflection of a cantilever before breaking is given by (4.19) where $S$ is the flexure strength, $E$ is Young's modulus, and $l$ and $h$ are the length and height of the cantilever, respectively.

$$
\delta_{\max }=\frac{2 S l^{2}}{3 E h}=8.41 \mu m
$$

This value is an approximate maximum tip deflection (assuming a parabolic profile) that an AIN cantilever with $l=120 \mu m$ and $h=1 \mu m$ can undergo before breaking. Therefore, a dc voltage of $\Delta V=40 \mathrm{~V}$ was chosen because the simulation results indicate a maximum deflection of $\delta_{\max (40 \mathrm{~V})}=2.31 \mu \mathrm{m}<\delta_{\max }=8.41 \mu \mathrm{m}$ and because this voltage is readily available in the laboratory. 
Finite element analysis was performed using the commercially available software tool ANSYS ${ }^{\circledR}$ Academic Research, v. 11.0. The model consisted of two SOLID226 coupled-field solid elements with their KEYOPTs set to 1001 (see Appendix G). This combination provided the model with displacement (UX, UY, UZ) and voltage (V) degrees of freedom (DOF). The elements were defined using two relative coordinate systems so that the polarization of the material would be opposing. The stiffness matrix coefficients, piezoelectric stress constants, dielectric permittivity, and density of AlN found in the literature were used with all values converted to $\mu \mathrm{MKSV}$ units [ANSYS, 2007; Gualtieri, 1994]. The model was meshed with hexahedral-shaped elements using mapped meshing. This produced a mesh with 30,545 nodes and zero error/warnings. The contact geometries simulated in this work are given in Tables 4.6-4.8. The electric potential plot implies the shape of the top contact used. The bottom contact geometry was not varied and was defined to cover the entire bottom of the cantilever due to fabrication limitations.

Table 4.6. $A N S Y S^{\circledR}$ Results Viewer plots of the electric potential and total strain intensity of the "Full Contact" and the "Half Contact".

\begin{tabular}{l|l|l|l|}
\hline Full Contact (FC) & Electric Potential & \multicolumn{2}{c}{ Total Strain Intensity } \\
\hline Half Contact (HC) & & & \\
\hline
\end{tabular}


Table 4.7. ANSYS ${ }^{\circledR}$ Results Viewer plots of the electric potential and total strain intensity of the "Diamond Shaped Contact" and the "T Shaped Contact".

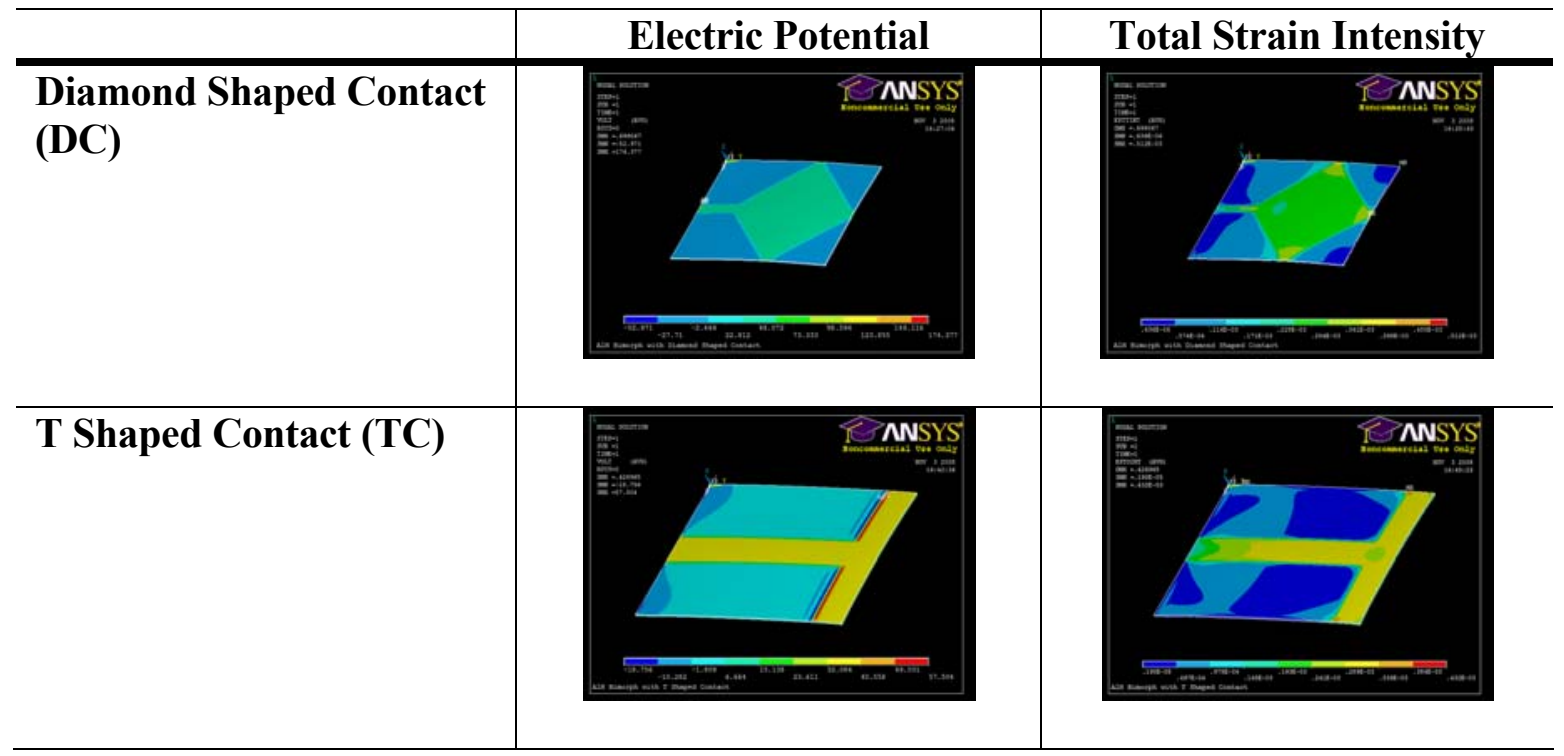

Table 4.8. $A N S Y S^{\circledR}$ Results Viewer plots of the electric potential and total strain intensity of the "Cantilever Shaped Contact" and "Vertical Contacts".

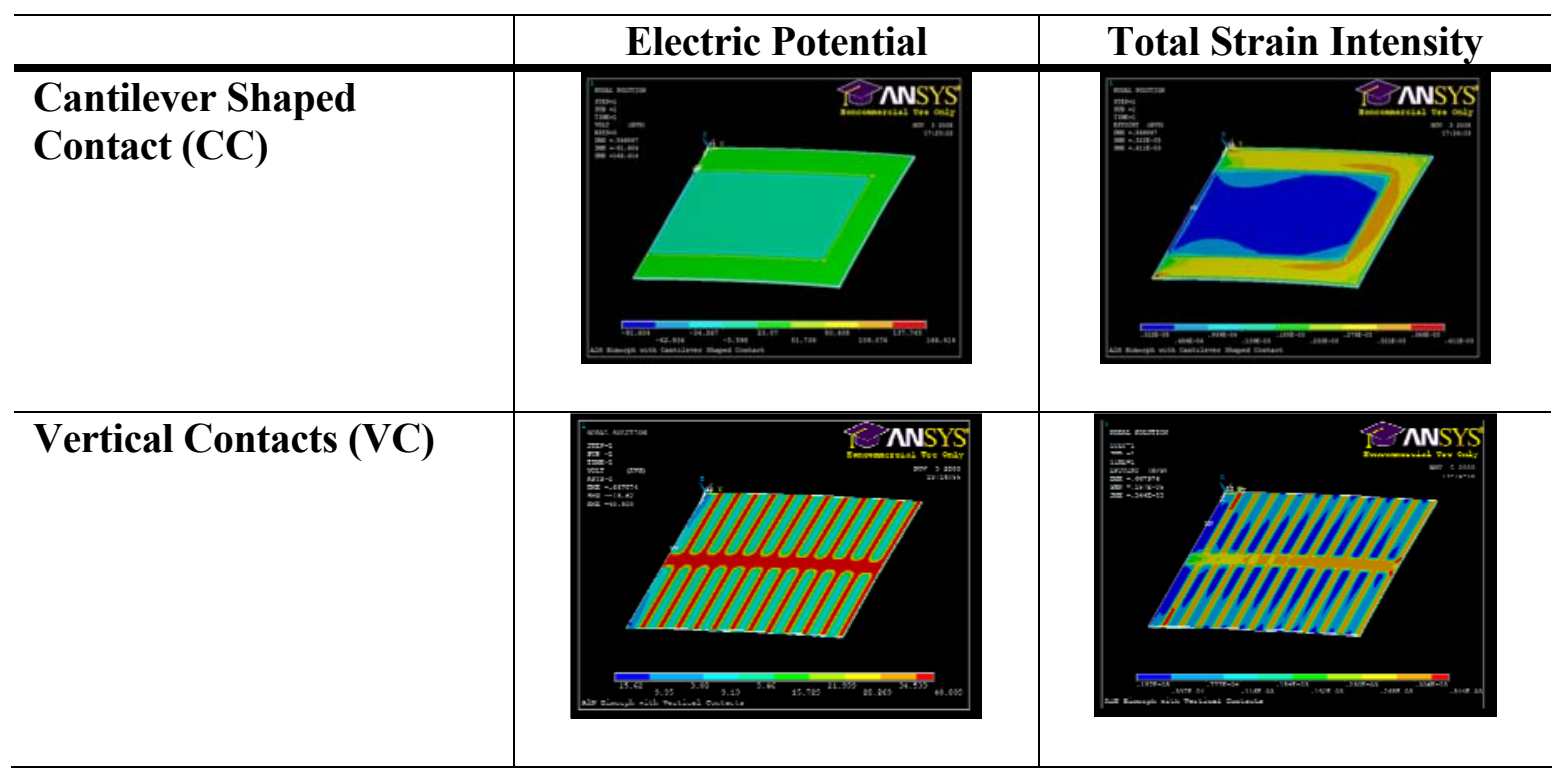


Table 4.9. ANSYS ${ }^{\circledR}$ Results Viewer plots of the electric potential and total strain intensity of "Vertical Contacts (4/3) $)^{n "}$ and "Vertical Contacts $(3 / 2)^{n}$ ".

\begin{tabular}{|c|c|c|}
\hline & Electric Potential & Total Strain Intensity \\
\hline $\begin{array}{l}\text { Vertical Contacts }(4 / 3)^{n} \\
(\mathrm{VC43)}\end{array}$ & $=-1$ & PANSYS \\
\hline $\begin{array}{l}\text { Vertical Contacts }(3 / 2)^{n} \\
(\text { VC32) }\end{array}$ & 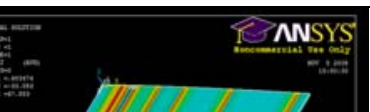 & 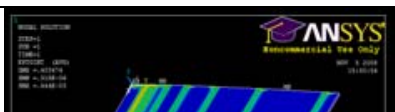 \\
\hline
\end{tabular}

The ANSYS $^{\circledR}$ Academic Research, v. 11.0 Results Viewer was used to plot the stress intensity, total mechanical strain intensity, electric potential, and displacement of the bimorph structures in 3- $d$. The electric potential and total mechanical strain intensity 3-d plots are shown in Tables 4.6-4.9. Three paths were defined and were named "Mid", "Edge", and "Tip" (see Figure 4.15). These paths correspond to ( $\mathrm{x}=w / 2, \mathrm{y}, \mathrm{z}=0),(\mathbf{x}=w$, $\mathrm{y}, \mathrm{z}=0)$ and $(\mathrm{x}, \mathrm{y}=l$, and $\mathrm{z}=0)$, respectively. The displacements in the $z$-direction of the cantilever along these paths for the simulated contact geometries are shown in Figure 4.164.18 


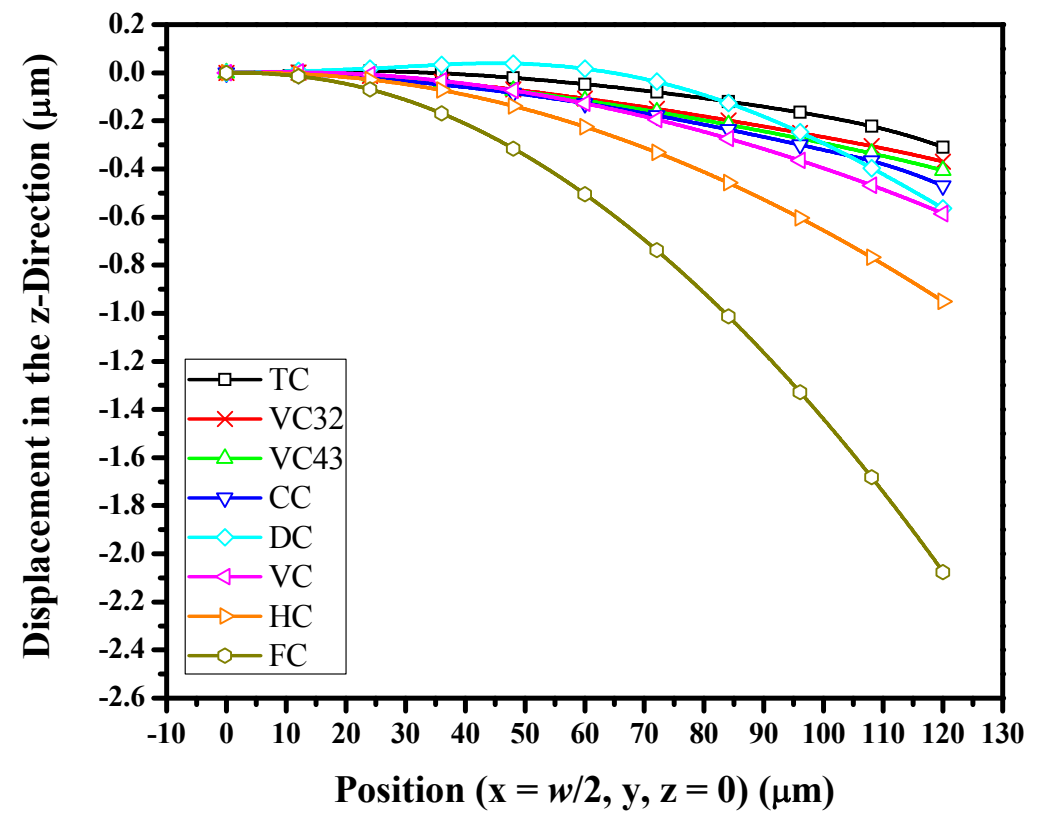

Figure 4.16. Bimorph deflection along the "Mid" path.

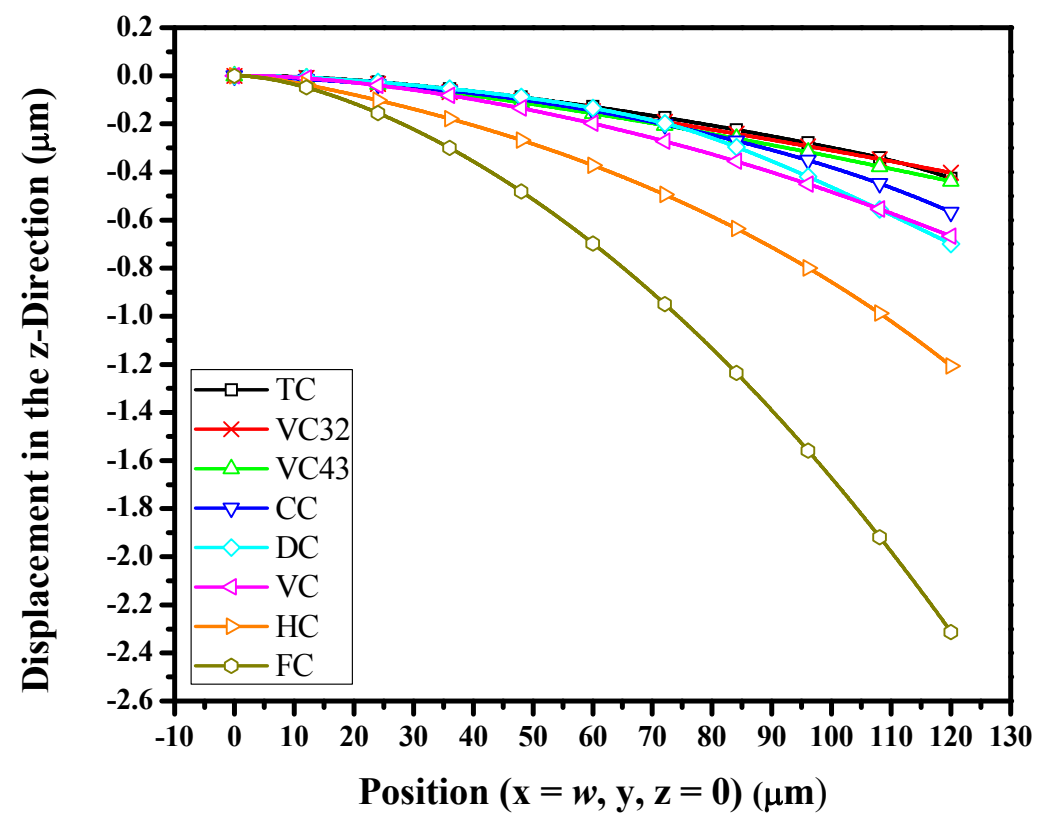

Figure 4.17. Bimorph deflection along the "Edge" path. 


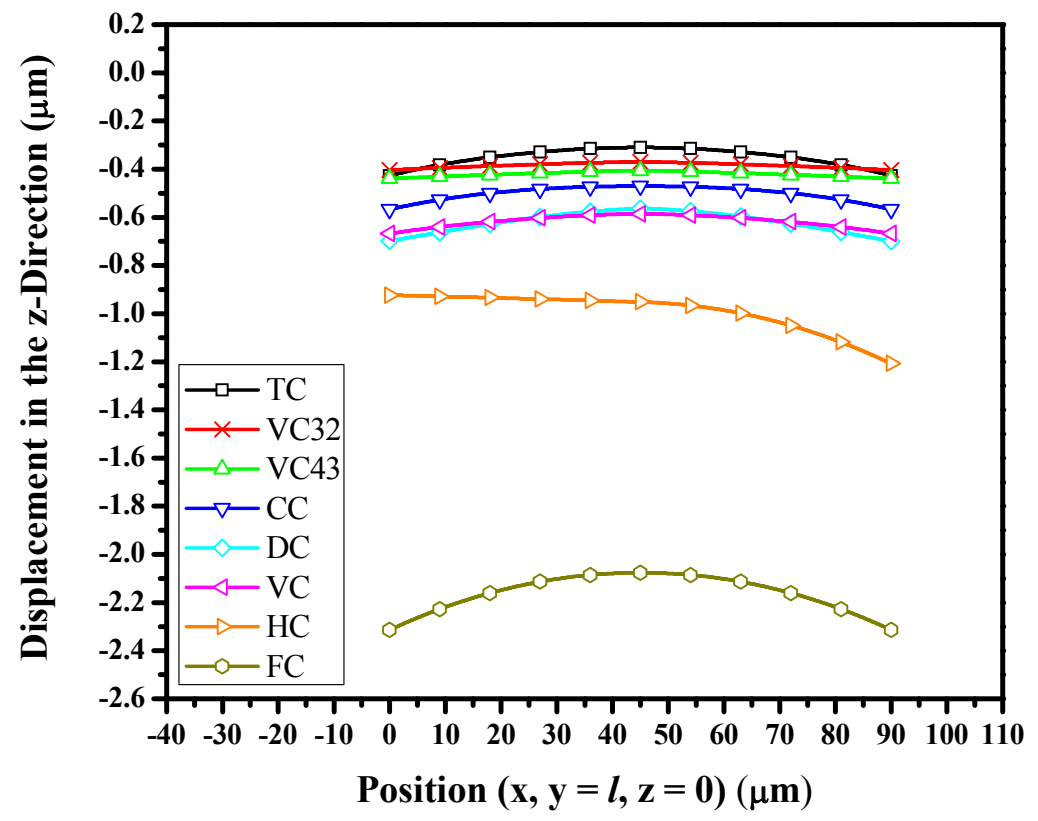

Figure 4.18. Bimorph deflection along the "Tip" path.

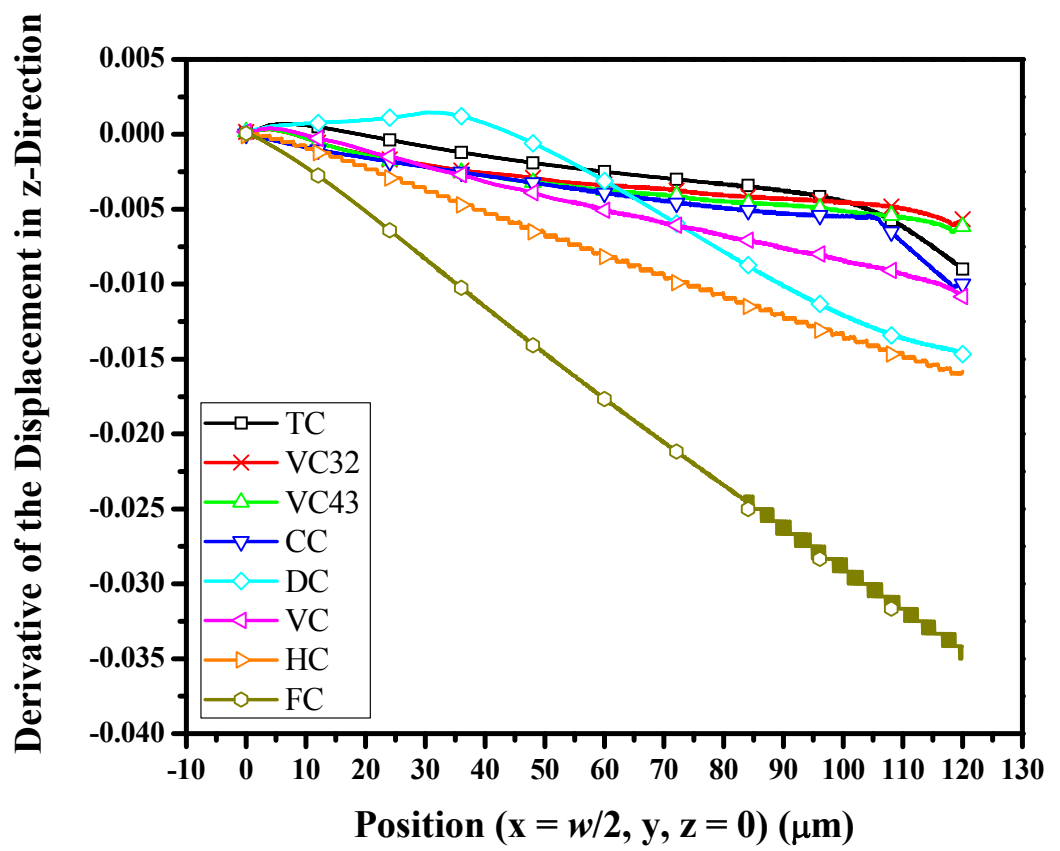

Figure 4.19. Derivative with respect to position of the deflection along the "Mid" path (see Figure 4.15(b)). 
It can be observed from Figures 4.16-4.17 that the displacement varies as a function of contact geometry. The maximum deflection at $\Delta V=40 \mathrm{~V}$ was obtained from the "Full Contact" while the minimum deflection was obtained from the "T Contact". The displacement profiles of the tip were $2^{\text {nd }}$ order for the "Full Contact" and "T Contact"; 3 rd order for the "Half Contact"; $4^{\text {th }}$ order for the "Cantilever Contact" and "Vertical Contact"; $6^{\text {th }}$ order for the "Diamond Contact"; and, $8^{\text {th }}$ order for the "Vertical Contacts $(4 / 3)^{n}$ " and "Vertical Contacts $(3 / 2)^{n}$ " as given by curve fitting with an adjusted $\mathrm{R}$ value $\geq 0.999$. Additionally, the derivative of the "Mid" path was taken to show the slope of the displacement as a function of position. Linear curves indicate parabolic deflection. The maximum values of deflection, stress, strain, and contact area found in this study are provided in Table 4.10 .

Table 4.10. Maximum values of the deflection, stress, strain and area for the contacts defined in Tables 4.6-4.9.

\begin{tabular}{c|c|c|c|c|c|c|c|c}
\hline & FC & HC & DC & TC & CC & VC & VC43 & VC32 \\
\hline $\begin{array}{c}\boldsymbol{\delta}_{\max } \\
(\mu \mathrm{m})\end{array}$ & 2.314 & 1.208 & 0.699 & 0.427 & 0.567 & 0.668 & 0.439 & 0.403 \\
\hline $\begin{array}{c}\boldsymbol{\sigma}_{\max } \\
(\mathrm{MPa})\end{array}$ & 1267 & 1233 & 707 & 480 & 1029 & 457 & 432 & 430 \\
\hline $\begin{array}{c}\boldsymbol{\varepsilon}_{\max } \\
\left(\mathrm{x} 10^{-3}\right)\end{array}$ & 0.684 & 0.626 & 0.512 & 0.432 & 0.411 & 0.344 & 0.428 & 0.444 \\
\hline $\begin{array}{c}\boldsymbol{A}_{\text {contact }} \\
\left(\mu \mathrm{m}^{2}\right)\end{array}$ & 10800 & 5400 & 4858 & 3253 & 4401 & 3733 & 2794 & 2547 \\
\hline
\end{tabular}

\subsubsection{SUMMARY}

This work has shown that the deflection of a piezoelectric bimorph in 3- $d$ is a function of contact geometry and position. The deflection profile can be optimized for a specific application by engineering the geometry of the top contact. In addition, freestanding $\mathrm{AlN} / \mathrm{SiO}_{2}$ diaphragms were fabricating using bulk micromachining. These structures hold promise for developing diaphragm based cantilever microvalves. 


\section{H A P T E R 5 : S U R F A E A C O U S T I C W V E D E V I C E S}

\subsection{BACKGROUND}

Acoustic waves have been used for many years to determine the mechanical properties of materials. For example, internal defects have been detected in forgings and castings for centuries by observing the ringing note generated when they are struck with a hammer [Krautkrämer, 1990]. Surface acoustic waves (SAWs) are a type of surface elastic waves (discussed in Section 5.1.1). Surface elastic waves propagate on the boundary between two mediums. All elastic solids can sustain surface elastic waves [Kallard, 1971]. However, the mode supported is a function of the material properties and the geometrical configuration of the mediums involved. As an example, seismic waves travelling along the earth's crust are surface elastic waves [Kallard, 1971]. SAWs can be used to determine the piezoelectric and elastic properties of a solid [Hickernell, 1996]. As an example, the most commonly used piezoelectric and elastic coefficients of AlN were determined by Tsubouchi et al. using SAWs [Tsubouchi, 1981]. Additionally, SAWs are highly sensitive to interactions at the interface between two mediums. Thus, SAW sensors are frequently used to develop chemical and biological sensors [Branch, 2004].

\subsubsection{ACOUSTIC WAVES}

Acoustic waves can be described as the periodic mechanical disturbances of particles composing the propagation medium. Acoustic waves are a subset of elastic waves as mentioned in Section 5.1. Elastic waves are mechanical waves in which restoring forces are proportional to the particles' displacements act to return the particles to their original positions. Elastic waves can only exist when the wavelength is much longer than the distance between the particles comprising the medium [Kallard, 1971; Krautkrämer, 1990]. Elastic waves can have particle motion in the direction of the wave propagation (i.e. a "longitudinal wave") or in a direction perpendicular to the direction of the wave propagation (i.e. a "transverse wave"). Longitudinal and transverse wave are shown in Figures 5.1 and 5.2, respectively. 


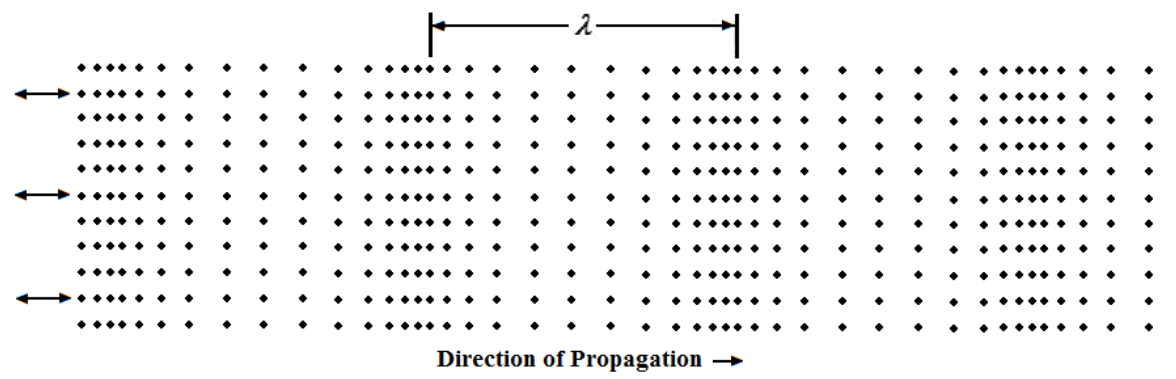

Figure 5.1. Longitudinal wave. After Krautkrämer and Krautkrämer [Krautkrämer, 1990].

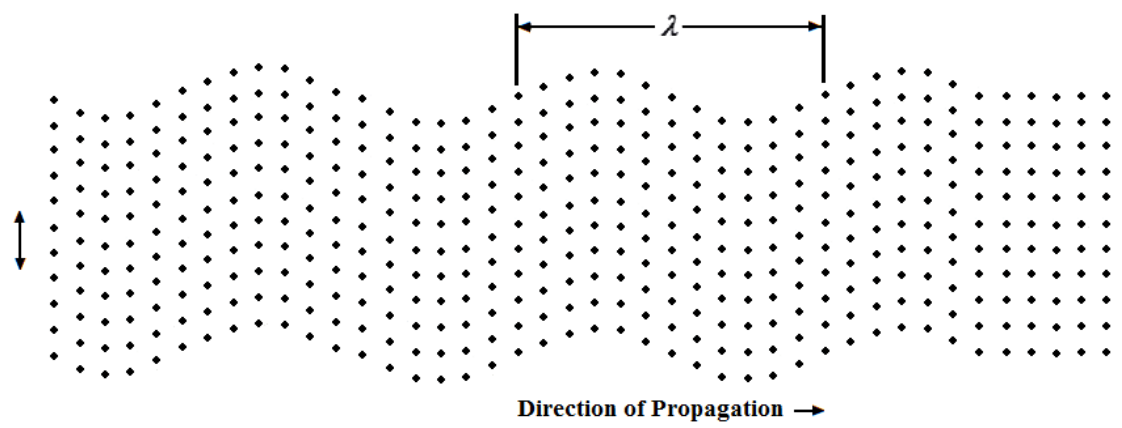

Figure 5.2. Transverse wave. After Krautkrämer and Krautkrämer [Krautkrämer, 1990].

\subsubsection{Acoustic Wave Modes}

Acoustic wave modes are classified by the wave velocities and displacement directions [Drafts, 2000]. The three modes commonly used in SAW sensors are Rayleighwave, Lamb-wave, and Love-wave [Gronewold, 2007]. The polarization of surface waves is often described in terms of the sagittal plane. This plane is defined by the surface normal and the propagation vector [Kallard, 1971]. For example, if the direction perpendicular to the surface is in the $-z$-dir and the direction of propagation is in the $x$-dir, the sagittal plane is the $x-z$ plane [Cheeke, 2002].

Rayleigh-waves are the simplest type of SAW. These waves propagate along the surface of a semi-infinite half space at a speed independent of their wavelength [Kallard, 1971]. Additionally, they are non-dispersive in mediums that lack mechanical dissipation. Lamb-waves are degenerate Rayleigh-waves that have been constrained in-plane. Thus, 
they are also known as flexural plate waves [Gronewold, 2007]. This occurs when the thickness perpendicular to the surface of the propagation medium is reduced [Krautkrämer, 1990]. Typical thicknesses of layers used to develop Lamb-waves are a few micrometers [Lee, 2005]. The particle displacement for Rayleigh-waves and Lamb-waves is out of plane. This means that energy can be transferred from the solid into the surrounding medium [Gronewold, 2007]. Thus, Rayleigh-wave and Lamb-wave mode SAW sensors are normally used for gas detection [Bryant, 1982; D’Amico, 1982; Gronewold, 2007]. However, since Rayleigh waves are not constrained to the interface, some of the mechanical energy is distributed in the substrate material [Gronewold, 2007].

The particle displacement for Love-waves is purely shear horizontal. Thus, little energy acoustic energy can be transferred to the surrounding medium making Love-waves the preferred mode for sensing in liquids [Kalantar-Zadeh, 2001]. Love-waves are typically generated in a waveguide layer having a shear acoustic velocity that is lower than that of the piezoelectric. Common waveguide layers include silicon dioxide and certain polymers [Branch, 2004]. Love-wave mode sensors are the most sensitive type of acoustic sensor [Gronewold, 2007]. Additionally, this mode offers the best SNR and system stability [Hur, 2005]. However, Love-waves are inherently dispersive [Branch, 2004].

\subsubsection{Surface Acoustic Waves}

Surface acoustic waves (SAWs) are acoustic wave that travels near the surface of the propagation medium. SAWs typically experience a strong reduction in amplitude as a function of distance from the surface [Kallard, 1971]. The penetration depth of the SAWs decrease as the frequency is increased. Thus, the surface sensitivity is increased as a function of the square of the frequency [Gronewold, 2007]. The wave velocity and the amplitude are parameters commonly measured to determine interactions such as mass loading, viscosity, and changes in conductivity [Gronewold, 2007; Länge, 2008].

\subsubsection{SAW MATERIALS}

The most desirable qualities for the piezoelectric material in a SAW device are a large electromechanical coupling coefficient, $k^{2}$, and high acoustic velocity, $v_{p}$ [Kao, 2002]. 
The coupling factor is a measurement of the electrical to mechanical efficiency of the material [Fansler, 2008] and can be expressed in terms of the free dielectric constant, $\varepsilon^{T}$, and the clamped dielectric constant, $\varepsilon^{S}$, as given in (5.1) [Jaffe, 1971].

$$
k^{2}=1-\frac{\varepsilon^{S}}{\varepsilon^{T}}
$$

Common piezoelectric materials for SAW devices include Zinc Oxide ( $\mathrm{ZnO})$ [Gorla, 1999; Kalantar-Zadeh, 2001], Lithium Niobate $\left(\mathrm{LiNbO}_{3}\right)$ [Shibata, 1995; Deger, 1998; Kao, 2002], Lithium Tatalate $\left(\mathrm{LiTaO}_{3}\right)$ [Hur, 2005], and aluminum nitride [Pearce, 1981; Tsubouchi, 1981; Hickernell, 1996; Fujiki, 2000; Fansler, 2008]. Additional materials for acoustic wave devices were tabulated previously by Gualtieri et al. [Gualtieri, 1994]. Of these materials, AlN is very attractive for high-frequency SAW devices due to its high acoustic velocity $\left(\mathrm{v}_{p}=5,607 \mathrm{~m} / \mathrm{s}\right)$ [Caliendo, 1993] and moderate piezoelectric coupling factor [Hickernell, 1996; Fujiki, 2000].

In addition, multi-layer structures are frequently used to take advantage of certain material properties of the constituent materials. For example, $\mathrm{AlN} / \mathrm{LiNbO}_{3}$ bi-layer structures were developed by Kao et al. to exploit the high coupling coefficient of $\mathrm{LiNbO}_{3}$ and the high acoustic velocity of AlN. The bi-layer structure was found to have a composite acoustic velocity of $4,200 \mathrm{~m} / \mathrm{s}$ and a coupling factor of $1.5 \%$ [Kao, 2002]. Diamond films have also been incorporated in SAW multi-layer structures [Nakahata, 1995; Benetti, 2005; Fansler, 2008]. Diamond has the highest acoustic velocity of all materials [Benetti, 2005]. Thus, the acoustic velocity (and thus the frequency) of SAW devices consisting of a piezoelectric material on a diamond interlayer can be increased for a given interdigitated transducer linewidth [Benetti, 2005; Fansler, 2008].

\subsubsection{SAW DEVICES}

Most SAW devices operate using ultrasonic waves. In a similar way that electromagnetic waves having frequencies higher than can be detected by the human eye are termed ultraviolet waves, acoustic waves having frequencies higher than can be 
detected by the human ear are termed ultrasonic waves [Krautkrämer, 1990]. Popular SAW devices include delay lines, pulse compression filters, and frequency filters [Kallard, $1971]$.

\subsubsection{SAW DELAY LINES}

Two port SAW delay lines consist of a transmitting IDT and a receiving IDT on a piezoelectric material as shown in Figure 5.3. Mass adsorbed between the IDTs on a SAW delay line generally results in a change in the SAW velocity [Welch, 1996]. Thus, SAW delay lines are frequently used as biological and chemical sensors by coating the area between the IDTs with a sensitive material [Branch, 2004].

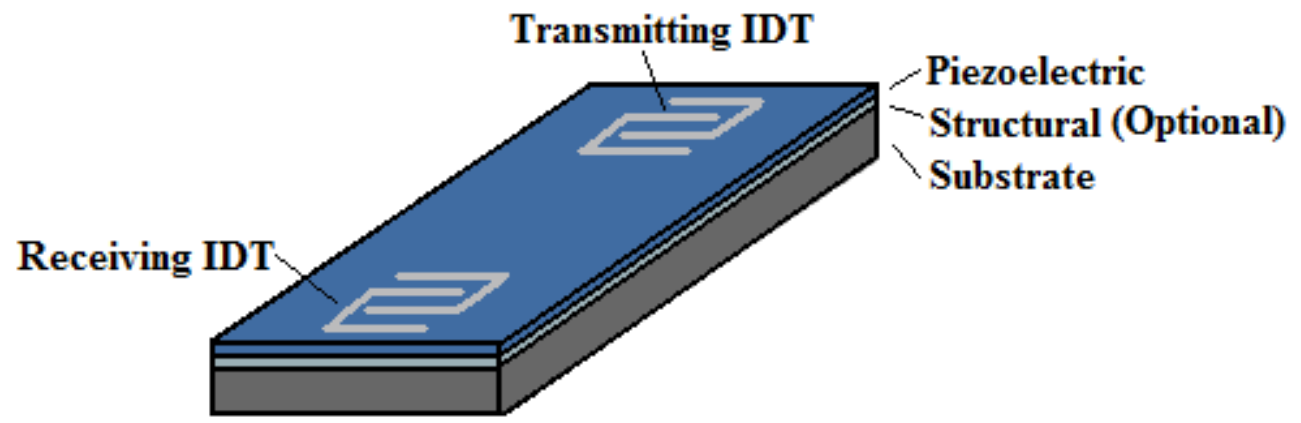

Figure 5.3. SAW two port delay line. After Pearce et al. [Pearce, 1981].

The delay time, $t_{d}$, is a function of the acoustic velocity of the materials composing the SAW, $v_{p}$, and the center-to-center distance, $d$, between the two IDTs as given in (5.2).

$$
t_{d}=d / v_{p}
$$

The SAW velocity is a function of the film thickness. For Rayleigh waves, the phase velocity decreases as the thickness is increased [Benetti, 2005]. Thus, the normalized thickness $(h / \lambda)$ or the normalized wavenumber $(k h)$ are commonly used. These two quantities differ by a factor of $2 \pi$ as shown in (5.3). 


$$
h k=h(2 \pi / \lambda)=2 \pi(h / \lambda)
$$

The use of a thickness independent quantity allows SAW devices with different thicknesses to be directly compared. Hickernell et al. determined the acoustic velocity in AIN SAW delay lines as a function of normalized thickness as shown in Figure 5.4. The AlN films were deposited on a $200 \mathrm{~nm}$ thick layer of silicon nitride. High quality AlN films had velocity dispersion curves that were similar to the theoretically predicted dispersion curve. Lower quality films had significantly lower velocities than theoretically predicted. The theoretical curve was determined using the coefficients reported by Tsubouchi et al. for epitaxial AlN [Hickernell, 1995].



AlN Film Thickness / Acoustic Wavelength

Figure 5.4. SAW velocity dispersion curves. (a) $2.8 \mu \mathrm{m}$ high quality AlN, (b) $1.0 \mu \mathrm{m}$ high quality AlN, (c) and (d)1.0 um lower quality AlN. After Hickernell [Hickernell, 1995].

\subsubsection{DIFFERENTIAL SENSORS}

Surface acoustic wave delay line sensors are also sensitive to environmental factors such as temperature and humidity. Thus, two identical delay lines can be used in parallel in order to eliminate environmental influences [Hur, 2005]. The area between one pair of IDTs (sensing channel) can be coated with a layer sensitive to the chemical or biological molecule to be detected [Benes, 1998]. Typically, the difference in frequency due to the 
difference in acoustic velocity between the sensing channel and the reference channel is measured. This is shown in Figure 5.5.

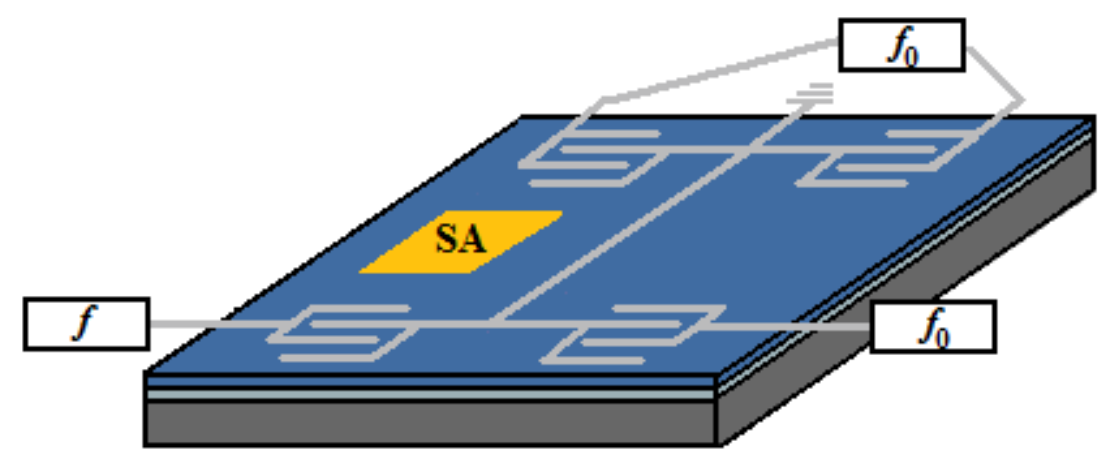

Figure 5.5. SAW differential sensor. The gold colored area in between the IDTs is the sensor area (SA). After Branch and Brozik [Branch, 2004].

A similar configuration to that shown in Figure 5.5 was used by Hur et al. to detect specific deoxyribonucleic acid (DNA) sequences. The differential delay line based SAW biosensor was fabricated using Lithium Tatanate $\left(\mathrm{LiTaO}_{3}\right)$ and exhibited a DNA hybridization sensitivity of $1.55 \mathrm{ng} / \mathrm{ml} / \mathrm{Hz}$ [Hur, 2005].

\subsubsection{ACOUSTOELECTRIC CONVOLVERS}

An acoustoelectric convolver can be fabricated by depositing a metal electrode in between the IDTs in a delay line SAW device. The output signal is then a function of the input signal applied to the transmitting IDT and the signal applied to the gate. These devices are used to convolve two signals together. A typical configuration for an acoustoelectric convolver is shown in Figure 5.6. A similar layout was fabricated and the results are discussed in Section 5.2.5. 


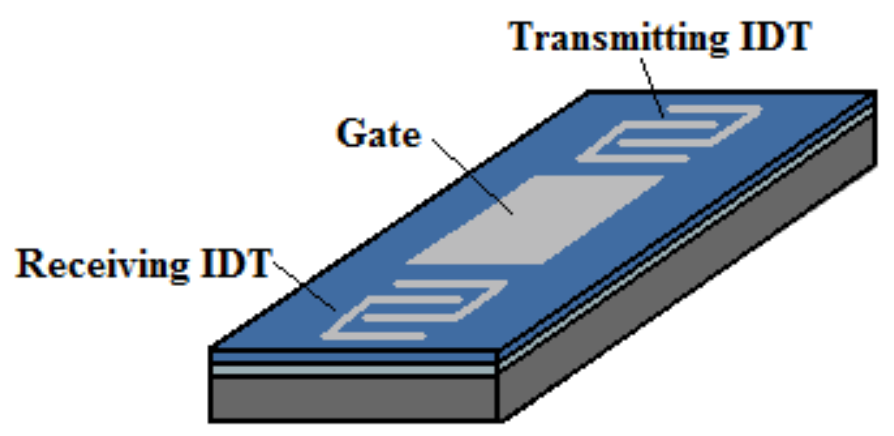

Figure 5.6. SAW acoustoelectric convolver. After Pearce et al. [Pearce, 1981].

\subsubsection{IDT POSITION}

The IDTs can be placed above or below the piezoelectric as shown in Figure 5.7. In the first case, the IDTs are located at the piezoelectric/substrate interface and in the second case, the IDTs are located on the surface of the piezoelectric.

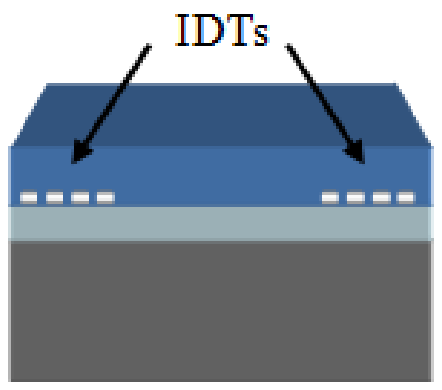

(a)

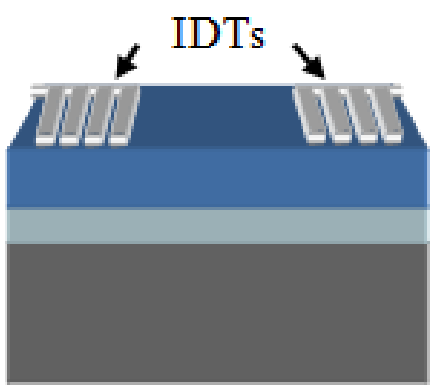

(b)

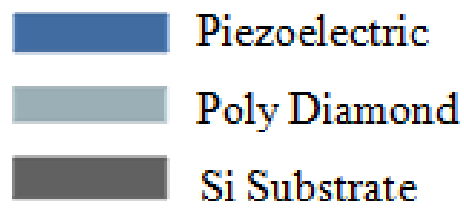

Figure 5.7. Two common IDT placements. After Nakahata et al. [Nakahata, 1995].

Nakahata et al. measured the acoustic velocities and electromechanical coupling coefficients of SAW delay lines using the placements shown in Figure 5.7. High velocities 
of over $10,000 \mathrm{~m} / \mathrm{s}$ were observed for $\mathrm{ZnO}$ films on diamond coated silicon wafers. For the $\mathrm{ZnO} / \mathrm{IDT} /$ diamond/ $\mathrm{Si}$ configuration, the acoustic velocity was $10,500 \mathrm{~m} / \mathrm{s}$ and the coupling coefficient was $1.5 \%$ (first mode). For the IDT/ $\mathrm{ZnO} /$ diamond/ Si configuration, the acoustic velocity was $11,600 \mathrm{~m} / \mathrm{s}$ and the coupling coefficient was $1.1 \%$ (second mode). It was also noticed that the IDT/ $\mathrm{ZnO} /$ diamond/ $\mathrm{Si}$ configuration did not show the second mode [Nakahata, 1995].

\subsection{ALN SAW DELAY LINES}

SAW delay lines hold much promise in the area of high sensitivity chemical and biological sensors. The design and testing of the SAW delay lines developed in this work are presented in this section.

\subsubsection{SAW DESIGN}

SAW devices use interdigitated transducers (IDTs) to generate and detect acoustic waves [Länge, 2008]. The characteristic frequency, $f_{0}$, generated by an IDT is a function of its line width, $w_{l}$, space width, $w_{s}$, and acoustic velocity, $v_{p}$. The characteristic frequency is the one in which wave excitation is the most efficient [Deger, 1998]. For the case where the line widths and the space widths are equal (i.e. $w_{l}=w_{s}=\lambda_{0} / 4$ ), the characteristic frequency can be related to the "finger" width, $w$, by (5.4) [Fansler, 2008].

$$
f_{0}=\frac{v_{p}}{4 w}=\frac{v_{p}}{\lambda_{0}}
$$

In this work, the line width, $w_{l}$, and space width, $w_{s}$, were designed to be $2 \mu \mathrm{m}$ since features smaller than these are difficult to obtain using photolithography. The IDT pattern used in this work is shown in Figure 5.8. Using the SAW velocity reported for $\operatorname{AlN}\left(v_{p}=\right.$ $5,607 \mathrm{~m} / \mathrm{s}$ ) [Caliendo, 1993], the characteristic frequency was calculated to be $700 \mathrm{MHz}$. 


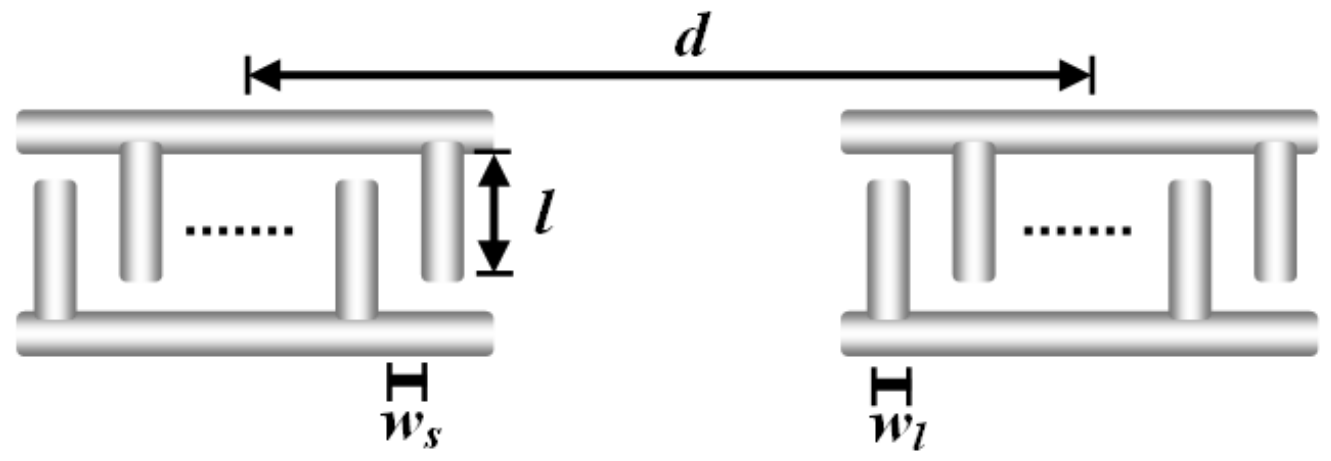

Figure 5.8. IDT pattern used in this work.

Using a similar IDT pattern to that shown in Figure 5.8 and using finger widths of 2 $\mu \mathrm{m}$, Benetti et al. experimentally found the characteristic frequency of AIN/ UNCD/ Si SAW delay lines to be $\sim 1 \mathrm{GHz}$ (see Section 3.2.1). This indicates that the acoustic velocity of the bi-layer structure was increased from approximately $5,600 \mathrm{~m} / \mathrm{s}$ to $8,200 \mathrm{~m} / \mathrm{s}$ without decreasing the finger width [Benetti, 2005].

The bandwidth (BW) of the main $\left|\mathrm{S}_{21}\right|$ lobe is a function of the characteristic frequency and the number of finger pairs, $N$, and can be expressed as (5.5) [Uehara, 2005].

$$
B W=\frac{2 f_{0}}{N}
$$

Thus, the bandwidth at a particular line and space can be (and thus frequency) can be varied by changing the number of finger pairs. The electromechanical coupling coefficient for SAW devices can be expressed in terms of the free velocity, $v_{f}$, and the electrically short-circuited velocity, $v_{m}$, as (5.6) [Bu, 2004].

$$
k^{2}=2\left(\frac{v_{f}-v_{m}}{v_{f}}\right)
$$


Additionally, the electromechanical coupling coefficient can also be explained in terms of the radiation conductance, $G_{a}$, and the susceptance, $B$, of the IDTs as given in (5.7) [Kao, 2002]

$$
k^{2}=\frac{\pi}{4 N} \times\left.\frac{G_{a}}{B}\right|_{f=f_{c}}
$$

In this work, four different IDTs were designed as given in Table 5.1. The spacing between the IDTs was either $180 \mu \mathrm{m}$ or $250 \mu \mathrm{m}$. (Note: the distance is from tip-to-tip and not from center-to-center).

Table 5.1. IDTs used in this work.

\begin{tabular}{c|c|c|c}
\hline IDT & Finger Length $(\boldsymbol{\mu m})$ & Number of Fingers & Number of IDT Pairs \\
\hline 3016 & 30 & 16 & $15(8,7)$ \\
\hline 3024 & 30 & 24 & $15(8,7)$ \\
\hline 4016 & 40 & 16 & $15(8,7)$ \\
\hline 4024 & 40 & 24 & $15(8,7)$ \\
\hline
\end{tabular}

\subsubsection{AIN DEPOSITION}

AlN films with nominal thicknesses of $300 \mathrm{~nm}$ were reactively sputtered using a CVC 610 DC magnetron sputter deposition system. The substrate used was a (100) p-type, double-side polished, 1-20 $\Omega$-cm resistivity silicon wafer purchased from Wafer World, Inc. (www.waferworld.com). Prior to loading the wafer in the sputtering chamber, the wafer was cleaned in a 5 minute acetone bath, a 5 minute methanol bath, and a 5 minute dip in 10:1 deionized (DI) water to J.T. Baker $49 \%$ hydrofluoric acid (HF). The wafer was rinsed in a cascading bath of DI water for 10 minutes, blown dry with nitrogen gas, and baked in a gravity oven for 10 minutes at $110^{\circ} \mathrm{C}$.

A $50 \mathrm{~mm}$-diameter, $99.999 \%$ pure Al target was to deposit the AlN film. Prior to deposition, the sputtering chamber was pumped to a base pressure below $5 \times 10^{-6}$ Torr using a CTI-8 cryopump. Since the Al target was known to oxidize when stored in an ambient environment, a two step pre-sputter was performed to remove the surface 
oxidation/contamination and coat the target with a thin layer of AlN. The pre-sputter was immediately followed by the film deposition. All pre-sputters and depositions were performed with a magnetron power of $100 \mathrm{~W}$ and a working pressure of $45 \mathrm{mTorr}$.

\subsubsection{IDT DEPOSITION}

Interdigitated transducers with a line and space of $2 \mu \mathrm{m}$ were deposited AlN film using image reversal photolithograpy. HexaMethylDiSilazane (HMDS) was spun onto the wafer for 30 seconds at $4000 \mathrm{rpm}$ to promote adhesion. Next, AZ5214-E photoresist was spun onto the wafer for 30 seconds at $4000 \mathrm{rpm}$ to form a $1.4 \mu \mathrm{m}$ thick layer of photoresist on the surface of the AlN film. The wafer was placed on a hotplate for 30 seconds at $95^{\circ} \mathrm{C}$ to remove excess solvents in the film. Once the photoresist was dried enough not to be sticky, the wafer was loaded into the mask aligner. The IDT pattern was transferred to the photoresist by exposing it through the mask to ultraviolet light. To perform image reversal, the exposed wafer was placed on a hotplate for 120 seconds at $120^{\circ} \mathrm{C}$ and then was exposed under an ultraviolet flood lamp for 82 seconds. The photoresist was developed in MIF-300 developer. The wafer was loaded into the sputtering chamber and platinum was sputtered onto the patterned side. The wafer was removed from the chamber and placed in a bath of acetone to perform lift-off. The remaining photoresist dissolved in the solvent leaving the IDTs behind. The wafer was cleaned in a 5 minute bath in acetone and then a 5 minute bath in methanol and was blown dry in nitrogen gas. The resulting structure is shown in Figure 5.9.

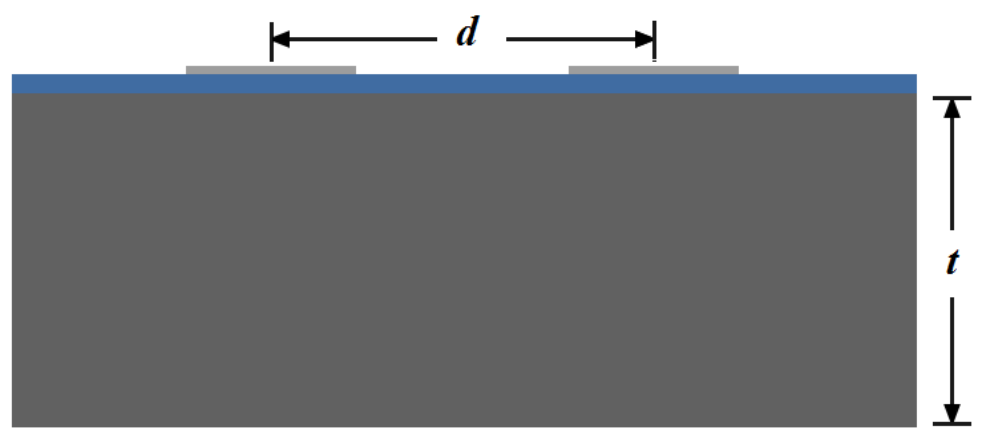

Figure 5.9. Rayleigh-wave mode $S A W$ delay line fabricated in this work. 
SEM images of the AlN devices were taken. Figure 5.10(a)-(b) shows IDTs in which all of the metal between the fingers was not completely removed during lift-off. The development and lift-off processes were found to be extremely sensitive to variations in the photolithography process. Thus, much care had to be taken to produce functional IDTs.

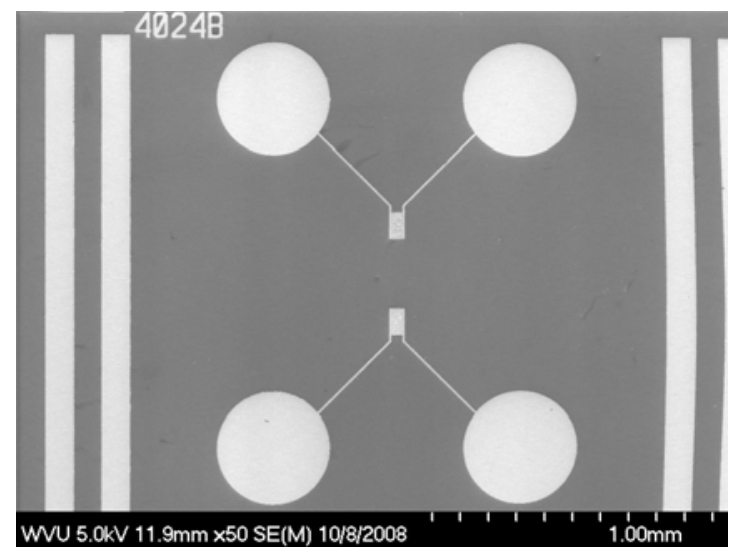

(a)

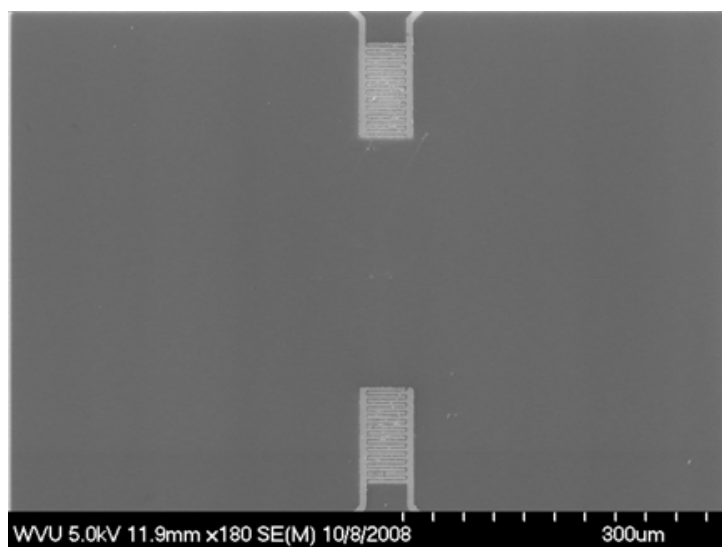

(b)

Figure 5.10. SEM images of an AlN SAW delay line with shorted IDTs. (a) 50 times magnification of entire device and IDTs and (b) 180 times magnification of IDTs.

Figure 5.11(a)-(b) shows a pair of IDTs that turned out well. The width of the platinum fingers can be seen to be approximately equal to the finger spacing. In addition, no shorts between IDT fingers were observed. 


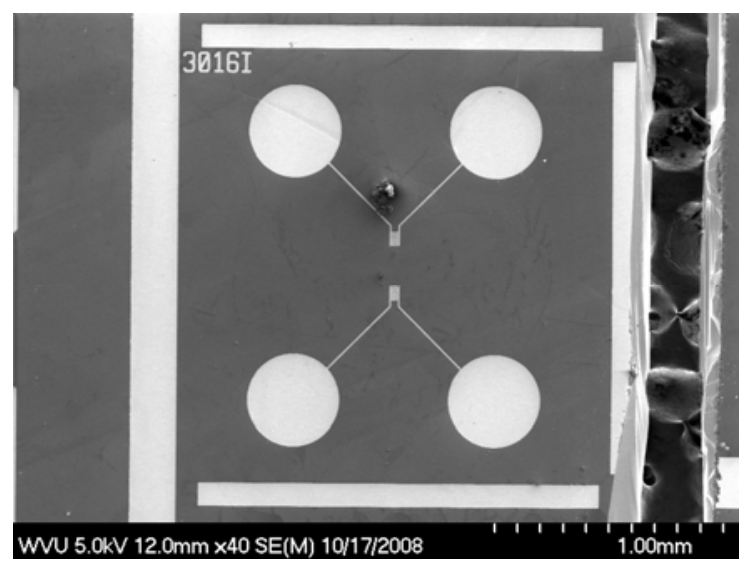

(a)

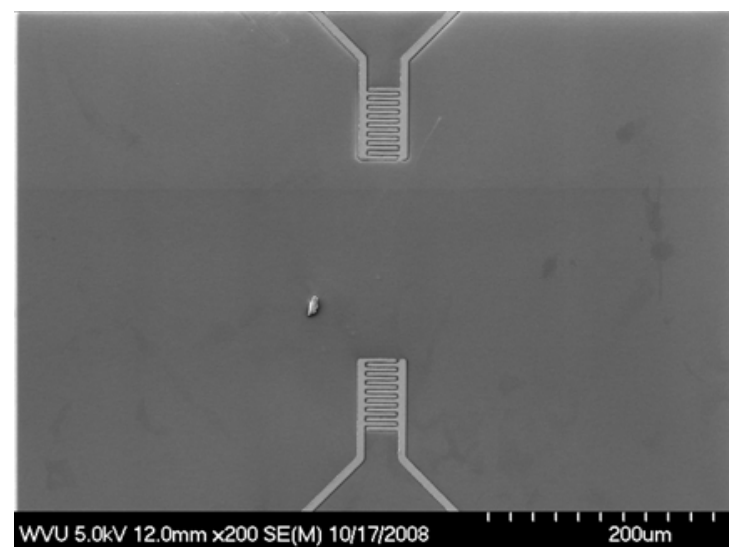

(b)

Figure 5.11. SEM images of AlN SAW delay line. (a) 40 times magnification of contacts and IDTs and (b) 200 times magnification of IDTs.

SEM images of the IDTs were also taken a magnification of $1 \mathrm{k}$ times as shown in Figure 5.12(a)-(b).



(a)

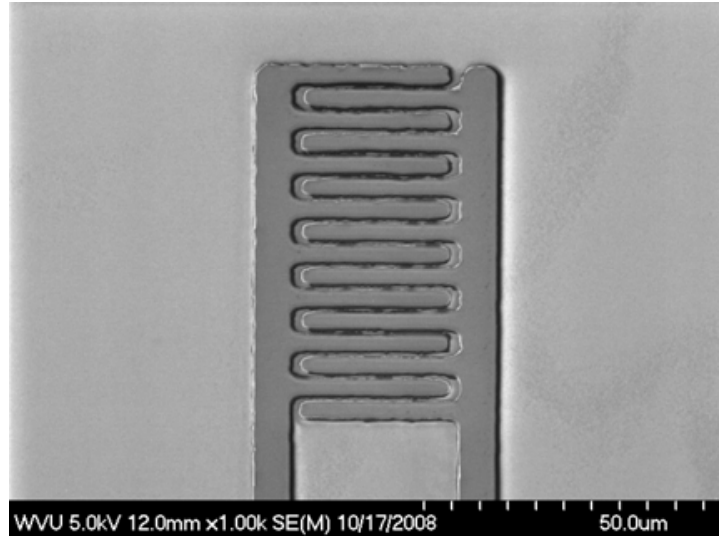

(b)

Figure 5.12. SEM images IDTs on AlN. (a) $1.00 \mathrm{k}$ times magnification of top IDT and (b) $1.00 \mathrm{k}$ times magnification of bottom IDT.

\subsubsection{DYNAMIC MEASUREMENTS}

Dynamic measurements were taken with a Tektronix AFG3102 function generator and Tektronix TDS2024B oscilloscope. Four Signatone S-745-CXM probes were used on a Signatone S-1160B-5N probe station. A sine of 5 periods, frequency of $50 \mathrm{MHz}$, and 
amplitude of $5 \mathrm{~V}$ was used. The IDT (4016I) had number of IDT pairs $=16$, finger spacing $=2 \mu \mathrm{m}$, finger length $=40 \mu \mathrm{m}$, and separation $=180 \mu \mathrm{m}$. The output signal (transmitted) and the input signal (received) are shown in Figure 5.13. The time delay between the two signals was used to calculate the phase velocity.

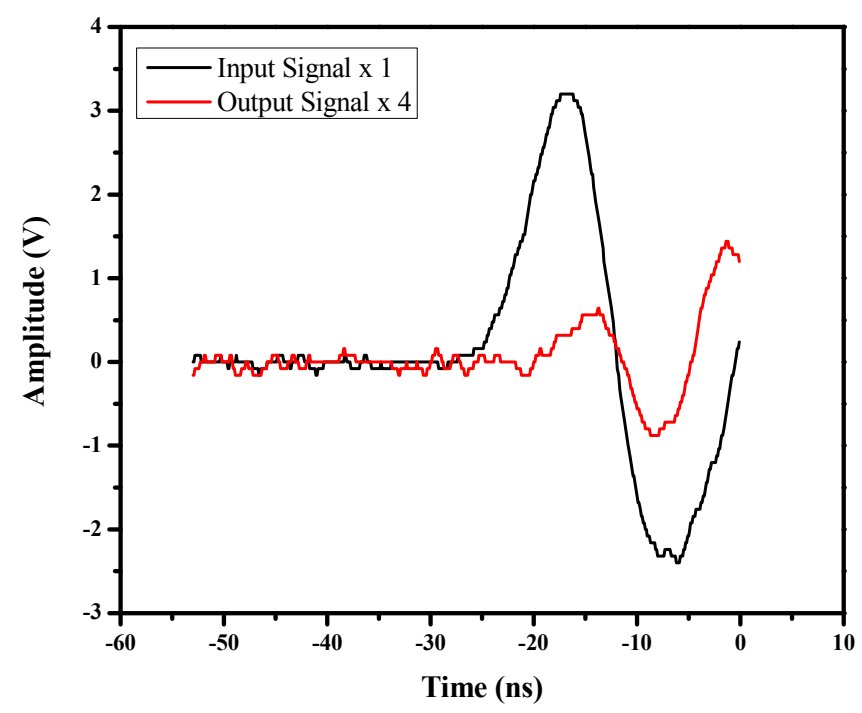

Figure 5.13. I/O response of a SAW delay line consisting of a $300 \mathrm{~nm}$ AlN thin film, freq $=$ $50 \mathrm{MHz}$.

Using the same setup as described previously, a pulse with period of $1 \mu \mathrm{s}$, duty cycle of $2 \%$, amplitude of $5 \mathrm{~V}$, and dc offset of $2.5 \mathrm{~V}$ was transmitted. The output signal (transmitted) and the input signal (received) are shown in Figure 5.14. The same plot is shown in Figure 5.15 with the $x$-axis range decreased. 


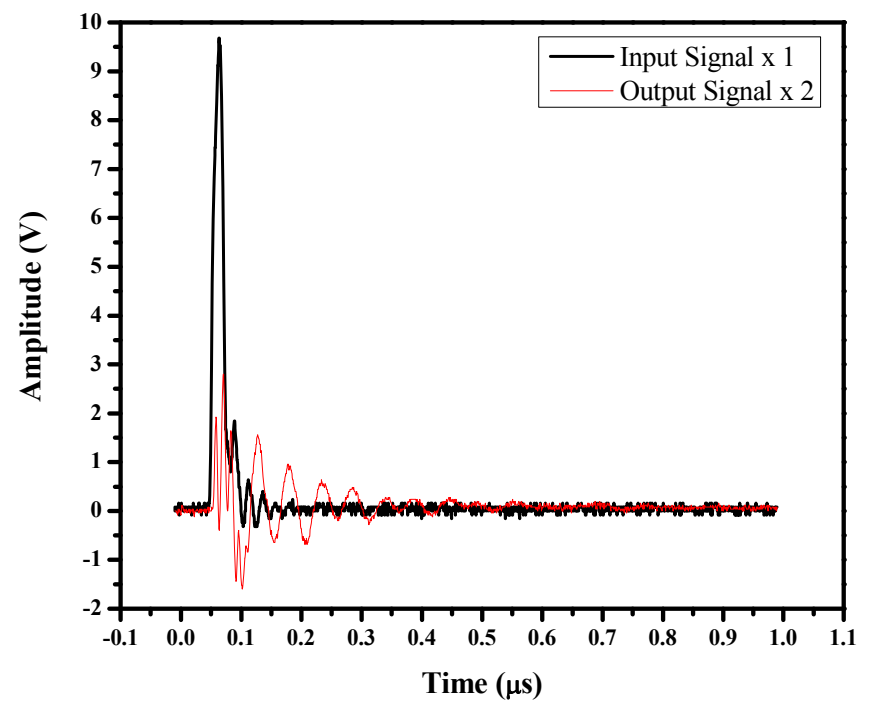

Figure 5.14. I/O response of a SAW delay line consisting of a $300 \mathrm{~nm}$ AlN thin film, $20 \mathrm{~ns}$ pulse.

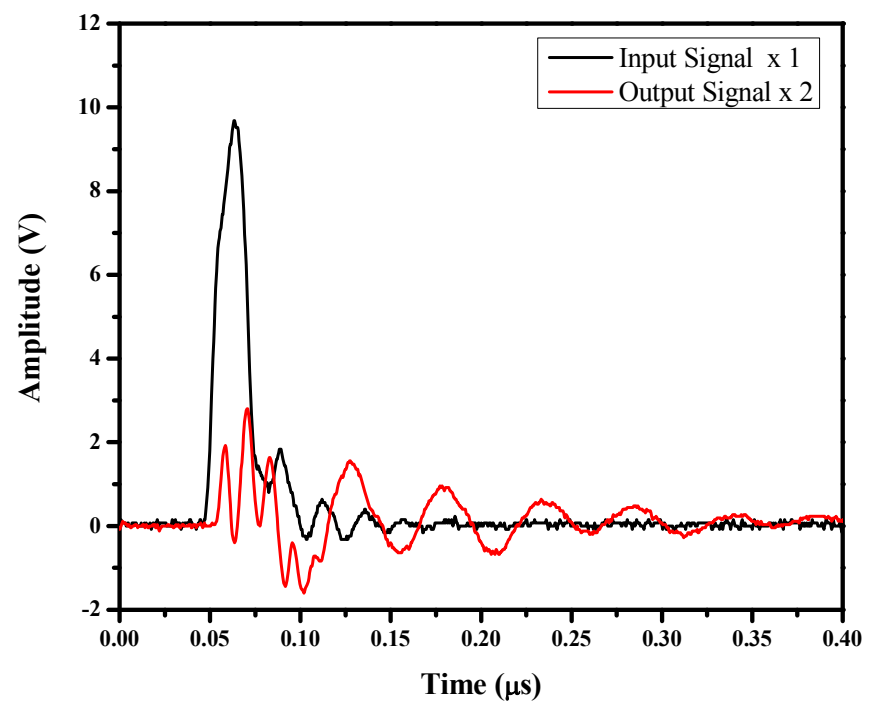

Figure 5.15. I/O response of a SAW delay line consisting of a $300 \mathrm{~nm}$ AlN thin film, $20 \mathrm{~ns}$ pulse (zoomed).

A continuous sine wave with frequency of $100 \mathrm{kHz}$ and amplitude of $5 \mathrm{~V}$ was used. The IDT (3016I) had number of IDT pairs $=16$, finger spacing $=2 \mu \mathrm{m}$, finger length $=30$ 
$\mu \mathrm{m}$, and separation $=180 \mu \mathrm{m}$. The output signal (transmitted) and the input signal (received) are shown in Figure 5.16.

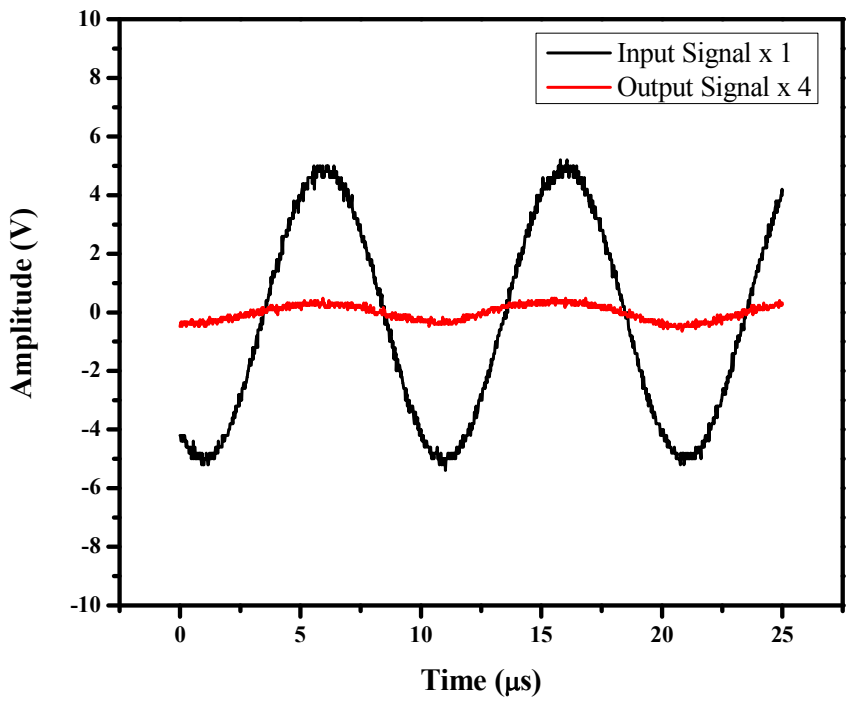

Figure 5.16. I/O response of a SAW delay line consisting of a $300 \mathrm{~nm}$ AlN thin film, freq $=$ $100 \mathrm{kHz}$.

The ratio of input signal to output signal, $A_{v}$, was determined from the $\mathrm{I} / \mathrm{O}$ data and was calculated to be 0.028 .

\subsubsection{Platinum Electrode StRIPS}

Platinum electrode strips with thicknesses of $70 \mathrm{~nm}$ were deposited between the IDTs. The distance between each of the IDTs and the platinum electrode was $15 \mathrm{~nm}$. The electrodes were deposited on SAW delay lines using a similar procedure to that used to deposit the IDTs. SEM images of the AIN SAW devices with platinum electrode strips are shown in Figure 5.17. 


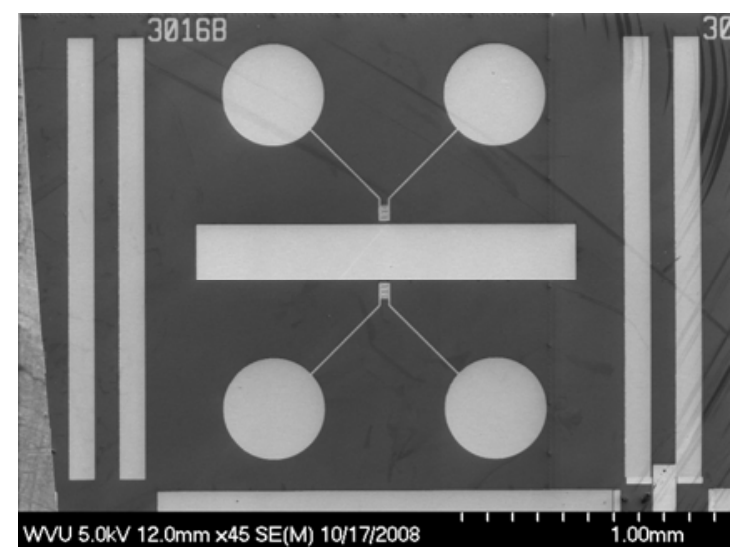

(a)

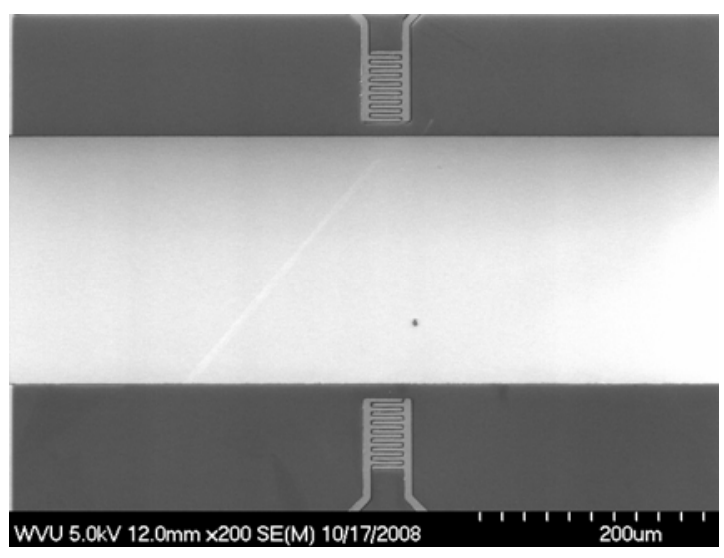

(b)

Figure 5.17. SEM images of an AlN SAW delay line with a platinum electrode strip. (a) 45 times magnification of entire device and (b) 200 times magnification of IDTs.

Using the same setup as described previously, a continuous sine wave with frequency of $100 \mathrm{kHz}$ and amplitude of 5V was used. The IDT (3016G) had number of IDT pairs $=16$, finger spacing $=2 \mu \mathrm{m}$, finger length $=30 \mu \mathrm{m}$, and separation $=250 \mu \mathrm{m}$. The output signal (transmitted) and the input signal (received) are shown in Figure 5.18.

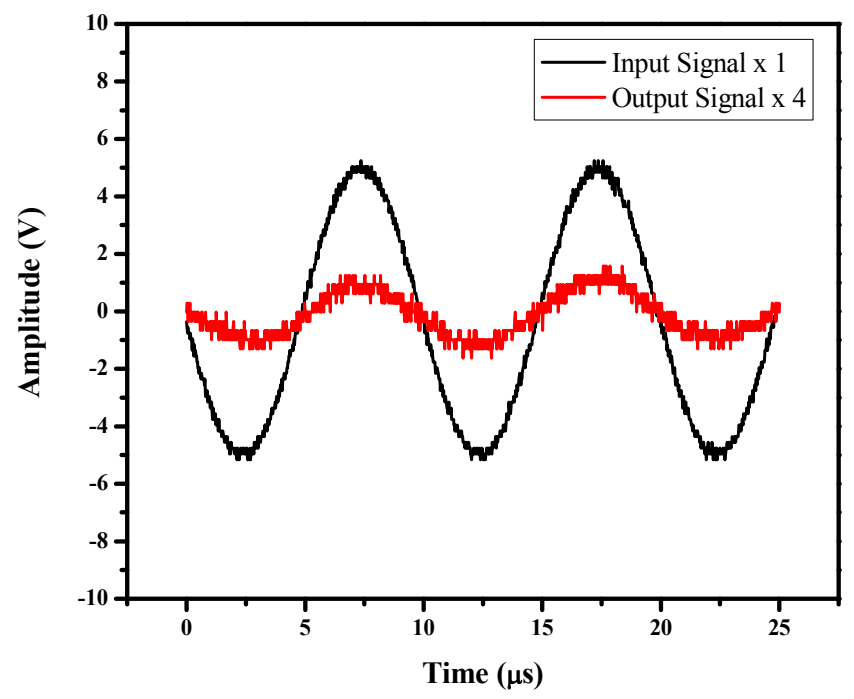

Figure 5.18. I/O response of a $S A W$ delay line consisting of a $300 \mathrm{~nm}$ AlN thin film and a $70 \mathrm{~nm}$ platinum electrode strip, $250 \mu \mathrm{m}$ separation, freq $=100 \mathrm{kHz}$. 
The ratio of input signal to output signal, $A_{v}$, was calculated for this measurement to be 0.0797 . Using the same setup as described previously, a continuous sine wave with frequency of $100 \mathrm{kHz}$ and amplitude of 5V was used. The IDT had (3016K) number of IDT pairs $=16$, finger spacing $=2 \mu \mathrm{m}$, finger length $=30 \mu \mathrm{m}$, and separation $=180 \mu \mathrm{m}$. The output signal (transmitted) and the input signal (received) are shown in Figure 5.19.

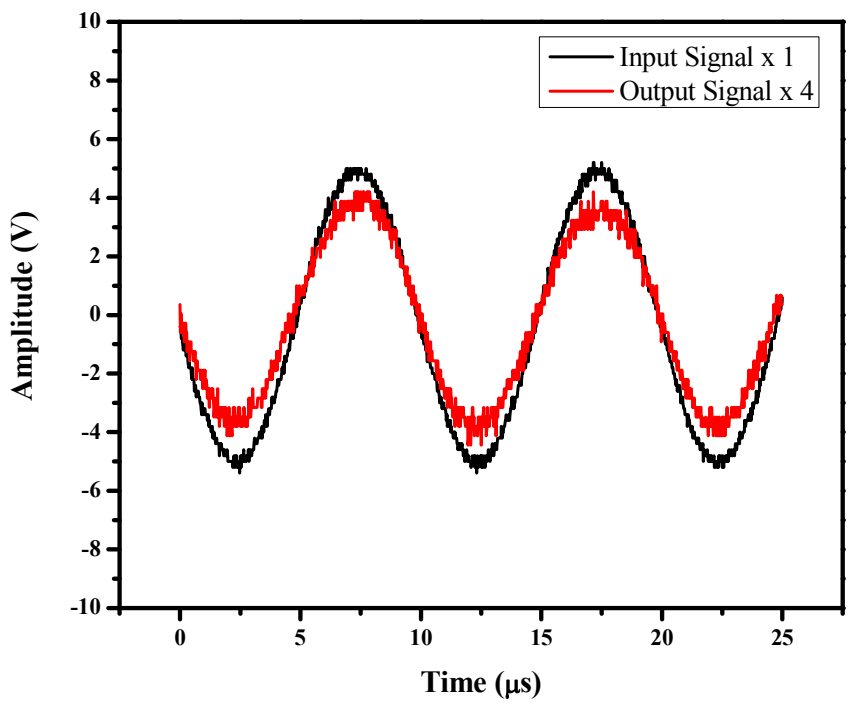

Figure 5.19. I/O response of a $S A W$ delay line consisting of a $300 \mathrm{~nm}$ AlN thin film and a $70 \mathrm{~nm}$ platinum electrode strip, $180 \mu \mathrm{m}$ separation, freq $=100 \mathrm{kHz}$.

The ratio of input signal to output signal, $A_{v}$, was calculated for this measurement to be 0.216 . The SAW delay line with a platinum electrode strip resembles an acoustoelectric convolver. To rule out the possibility that the increased response was due to a bias on the electrode, measurements were also taken with the strip grounded $(0 \mathrm{~V})$ and biased $(25 \mathrm{~V})$. However, the output signal was approximately the same for both biases as shown in Figure 5.20 . 


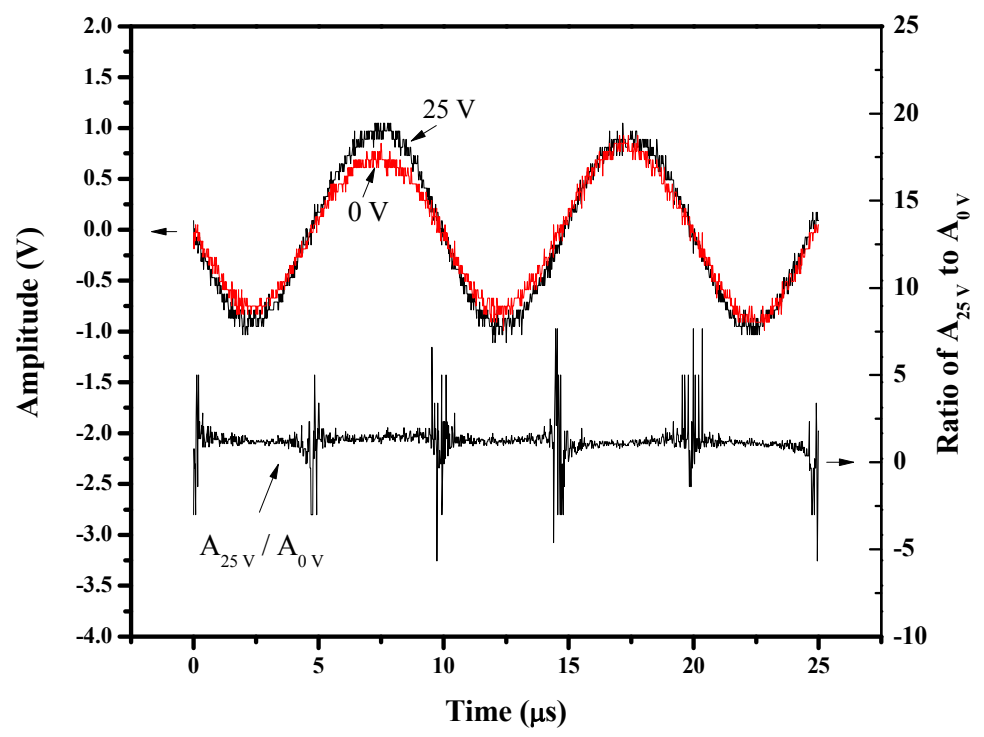

Figure 5.20. I/O response of a SAW delay line consisting of a $300 \mathrm{~nm}$ AlN thin film and a $70 \mathrm{~nm}$ platinum electrode strip, $180 \mu \mathrm{m}$ separation, freq $=100 \mathrm{kHz}$. The sinusoidal signals are the normalized output when the platinum strip is biased at $0 \mathrm{~V}$ and $25 \mathrm{~V}$. The ratio of these signals is also plotted.

The calculated gain for these three cases is plotted in Figure 5.21. It is believed that decrease in attenuation is due, at least in part, by the built-in electric field produced in the AlN film by the Pt strip (see Section 3.1.2.2). Further work needs to be performed to ensure that the lower attenuation is not accounted for solely by the change in the electromechanical coupling coefficient due to differences in the free and short-circuited velocities. 


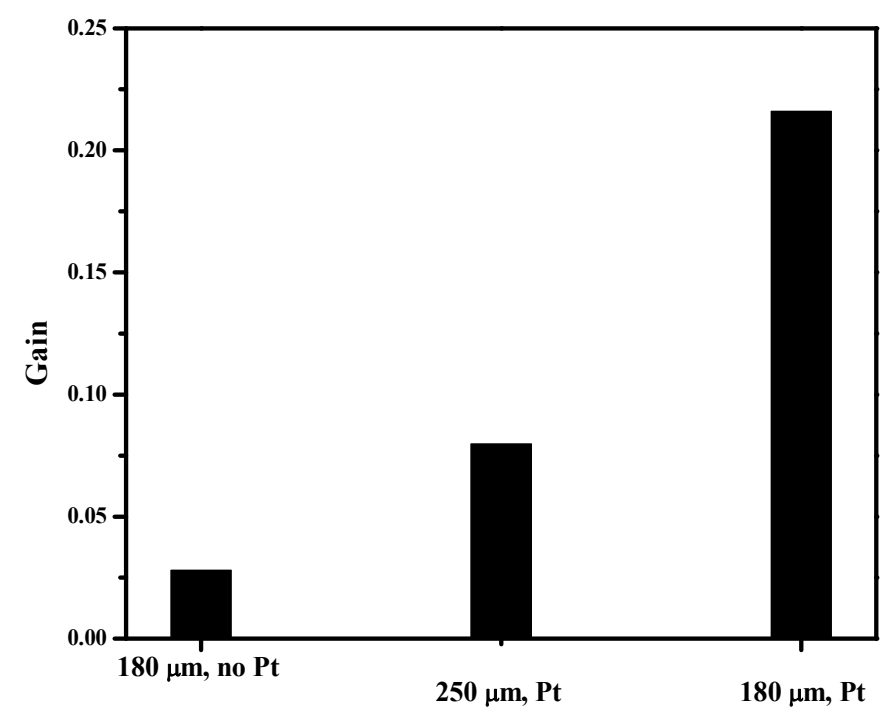

Figure 5.21. Comparison of SAW devices with and without a platinum electrode strip.

\subsubsection{SUMMARY}

The development of highly sensitive and selective chemical and biological sensors is of great importance for medical diagnosis and for biosecurity. Surface acoustic wave sensors are known to be very sensitive to their environment [Hur, 2005]. As discussed previously, SAW sensors can be fabricated by coating the region between the IDTs in a delay line SAW sensor with a layer sensitive to the chemical or biological substance to be detected. One of the advantages of using acoustic wave sensors for the detection of biomolecules is that they do not need to be labeled prior to being detected [Hur, 2005]. These devices have been used to detect proteins, nucleic acids, whole cells, membranes and vesicles, and polymers, among others [Gronewold, 2007].

In this work, AIN surface acoustic wave delay lines have successfully been fabricated. Additionally, a method for increasing the amplitude of the output signal was reported. In delay line SAW sensors, the waves traveling between the IDTs are affected by events occurring on the detection area. The input and output electrical signals are compared and the phase change and/or frequency change are correlated to a change in mass or a change in the mechanical properties [Gronewold, 2007]. AlN SAW platforms have 
much promise in the development of chemical and biological sensors due to AlN's high velocity. 


\section{CONCLUSIONS AND FUTURE DIRECTIONS}

In this work, the deposition parameters for reactively sputtering AlN thin films were optimized. Undoped and Er-doped AlN films were characterized using a variety of techniques including VASE ${ }^{\circledR}$, AFM, LDV, and XPS. C-V measurements were performed on AIN MIS structures to better understand the mechanism behind the piezoelectric enhancement found when using high work function metal contacts. Preliminary results suggest that the internal electric field in the AIN layer is indeed influenced by the contact metal used. Ultrananocrystalline diamond thin films show promise for increasing the performance of SAW devices for a given IDT linewidth. Therefore, UNCD films were characterized using $\mathrm{VASE}^{\circledR}$ and AFM. However, the UNCD films were found to have moderate surface roughness which may limit their use in SAW devices.

Free-standing $\mathrm{AlN} / \mathrm{SiO}_{2}$ and $\mathrm{Si}$ diaphragms were fabricated using bulk micromaching. The diaphragms were found to be robust; thus, they should be useful in the development of diaphragm based microvalves. AlN bimorph structures were simulated using ANSYS ${ }^{\circledR}$ 11.0. The deflection of the beam was found to be a function of contact geometry and position. As expected, the maximum tip deflection was produced when contacts spanning the entire length and width of the bimorph were used. However, the deflection along the "Mid" path, "Edge" path, and "Tip" path were noticeably different between the simulated contact geometries.

AlN SAW delay lines were developed and tested at low frequencies $(<50 \mathrm{MHz})$. The measured results agreed with the theoretical predictions. A reduction in attenuation was observed when a Pt electrode strip was deposited between the two IDTs. It is believed that the seven-fold enhancement in amplitude is due, at least in part, to the internal electric field developed across the AlN film when the Pt layer is present. A high frequency setup (shown in Appendix C) was designed and assembled. This setup has the capability of measuring the response of the SAW delay lines at frequencies between $20 \mathrm{MHz}$ to $20 \mathrm{GHz}$ in a low noise environment. The measurements should allow the material properties (such as piezoelectric coefficients, stiffness/compliance coefficients, acoustic velocity, etc.) to be extracted. 
Future directions are numerous. Of particular interest is the development of Lambwave mode SAW delay lines. This mode can provide increased sensitivity in comparison to the Rayleigh-wave mode. To fabricate devices of this type, it is necessary to reduce the thickness of the substrate material. One method is to combine the results in this work on free-standing diaphragms with the work on the Rayleigh-wave mode SAW delay lines. Furthermore, e-beam lithography can be used to create IDTs with finger widths much smaller than possible with conventional photolithography allowing higher frequency (and thus higher sensitivity) SAW devices to be developed. In addition to higher frequency operation, a lower SAW insertion loss is desirable. It has been observed that platinum may reduce the SAW attenuation through the delay line. Preliminary results also indicate that Er-doped AlN films also have a lower insertion loss than their undoped counterparts. Thus, it would be interesting to pursue these topics and possibly combine them to see if the insertion loss can be further reduced.

Finally, AlN bimorphs were simulated for the static case (dc). However, it is not known if the resonant frequency of the cantilever will change as a function of contact geometry when excited with an ac signal. Clearly, the reduced mass of contacts with a surface area less than complete coverage will lead to an increase in the resonant frequency. But, it is not understood if driving the piezoelectric using these different electrode configurations will affect the bimorphs resonant frequencies. Thus, this topic is also of interest. 


\section{BIBLIOGRAPHY}

[Adam, 2001] T. Adam, J. Kolodzey, C. P. Swann, M. W. Tsao, and J. F. Rabolt, Applied Surface Science, 175-176 (2001)

[Agilent Technologies, 2009] Agilent Technologies, "Agilent | Application Notes: E8362B PNA Series [Discontinued | Currently Supported]," Accessed Online April 6, 2009.

$<\mathrm{http}: /$ www.home.agilent.com/agilent/techSupport.jspx?pid=72279\&pageMode=AN\&cc= US\&lc $=$ eng $>$

[Akiyama, 2004] M. Akiyama, K. Nagao, N. Ueno, H. Tateyama, T. Yamada, Vacuum, 74 (2004)

[Aldraihem, 2003] O. J. Aldraihem and A. A. Khdeir, Composite Structures, 60, 2 (2003)

[Allen, 2004] R. Allen, Electronic Design (2004)

[Andrei, 2008] A. Andrei, K. Krupa, M. Jozwik, P. Delobelle, L. Hirsinger, C. Gorecki, L. Nieradko, and C. Meunier, Sensors and Actuators A, 141 (2008)

[ANSYS, 2007] ANSYS ${ }^{\circledR}$ Academic Research, Release 11.0, Help System, Elements Reference, ANSYS, Inc. (2007)

[Ashcroft, 1976] N. W. Ashcroft and N. D. Mermin, "Solid State Physics," Thomson Learning (1976)

[Assouar, 2004] M. B. Assouar, O. Elmazria, M. Elhakiki, and P. Alnot, The Journal of Vacuum Science and Technology B, 22, 4 (2004)

[Baborowski, 2004] J. Baborowski, Journal of Electroceramics, 12 (2004)

[Benes, 1998] E. Benes, M. Gröschl, and F. Seifert, IEEE Transactions on Ultrasonics, Ferroelectrics, and Frequency Control, 45, 5 (1998)

[Benetti, 2005] M. Benetti, D. Cannatà, F. D. Pietrantonio, and E. Verona, IEEE

Transactions on Ultrasonics, Ferroelectrics, and Frequency Control, 52, 10 (2005)

[Benetti, 2007] M. Benetti, D. Cannatà, F. D. Pietrantonio, and E. Verona, Proceedings of the IEEE Ultrasonics Symposium (2007)

[Bernhardt, 2002] P. V. Bernhardt, B. M. Flanagan, M. J. Riley, and B. J. Wood, Journal of Electron Spectroscopy and Related Phenomena, 124 (2002) 
[Boeshore, 2005] S. E. Boeshore, E. R. Parker, V. Lughi, N. C. MacDonald, and M. Bingert, Proceedings of the IEEE Ultrasonics Symposium (2005)

[Branch, 2004] D. W. Branch and S. M. Brozik, Biosensors and Bioelectronics, 19 (2004)

[Brien, 2007] V. Brien, P. Miska, H. Rinnert, D. Genève, and P. Pigeat, Materials Science and Engineering $B$ (2008)

[Bryant, 1982] A. Bryant, M. Poirier, D. L. Lee, and J. F. Vetelino, $36^{\text {th }}$ Annual Frequency Control Symposium (1982)

[Bu, 2004] G. Bu, D. Ciplys, M. Shur, L. J. Schowalter, S. Schujman, R. Gaska, Applied Physics Letters, 84, 23 (2004)

[Caliendo, 1993] C. Caliendo, G. Saggio, P. Veradi, and E. Verona, Proceedings of the IEEE Ultrasonics Symposium (1993)

[Campbell, 2001] S. A. Campbell, "The Science and Engineering of Microelectronic Fabrication," Oxford University Press (2001)

[Carlisle, 2003] J. A. Carlisle and O. Auciello, "Ultrananocrystalline Diamond Properties and Applications in Biomedical Devices," Interface (2003)

[Carlotti, 1995] G. Carlotti, F. S. Hickernell, H. M. Liaw, L. Palmieri, G. Socino, and E. Verona, Proceedings of the IEEE Ultrasonics Symposium (1995)

[Carrillo, 2003] D. Carrillo and K. Robinson, Surface Mount Technology (2003)

[Cascade Microtech, 2007a] Cascade Microtech, Inc., "MPH Microprobe Holder Technical and Ordering Information," (2007)

[Cascade Microtech, 2007b] Cascade Microtech, Inc., "CASCADE MICROTECH 2007," (2007)

[Cascade Microtech, 2009a] Cascade Microtech, Inc., "WinCal XE Specification Sheet," (2009)

[Cascade Microtech, 2009b] Cascade Microtech, Inc., Accessed Online May 6, 2009. $<$ http://www.cmicro.com/products/engineering-probes/rf-microwave/acp-probe/aircoplanar-probe-series $>$

[Chapuis, 2006] F. Chapuis, F. Bastien, J. F. Manceau, F. Casset, and P. L. Charvet, IEEE $7^{\text {th }}$ Int. Conf. on Thermal, Mechanical and Multiphysics Simulation and Experiments in Micro-Electronics and Micro-Systems, EuroSimE (2006) 
[Cheeke, 2002] J. D. N. Cheeke, "Fundamentals and Applications of Ultrasonic Waves," CRC Press (2002)

[Cheng, 2003] H. Cheng, Y. Sun, and P. Hing, Thin Solid Films, 434 (2003)

[Chowdhury, 1997] E. A. Chowdhury, J. Kolodzey, J. O. Olowolafe, G. Qiu, G. Katulka, D. Hits, M. Dashiell, D. van der Weide, C. P. Swann, and K. M. Unruh, Applied Physics Letters, 70, 20 (1997)

[Clement, 2003] M. Clement, E. Iborra, J. Sangrador, A. Sanz-Hervas, L. Vergara, and M. Aguilar, Journal of Applied Physics, 94, 1 (2003)

[D'Amico, 1982] A. D'Amico, A. Palma, and E. Verona, Applied Physics Letters, 41, 3 (1982)

[Deger, 1998] C. Deger, E. Born, H. Angerer, O. Ambacher, M. Stutzmann, J.Hornsteiner, E. Riha, and G. Fischerauer, Applied Physics Letters, 72, 19 (1998)

[Dogheche, 1999] E. Dogheche, D. Remiens, A. Boudrioua, and J. C. Loulergue, Applied Physics Letters, 74, 9 (1999)

[Dorenbos, 2006] P. Dorenbos and E. van der Kolk, Applied Physics Letters, 89, 061122 (2006)

[Drafts, 2000] B. Drafts, Sensors (2000)

[Dubois, 1998] M.-A. Dubois, P. Muralt, H. Matsumoto, and V. Plessky, Proceedings of the IEEE Ultrasonics Symposium (1998)

[Dubois, 1999] M.-A. Dubois and P. Muralt, Applied Physics Letters, 74, 20 (1999)

[Dubois, 2001] M.-A. Dubois and P. Muralt, Applied Physics Letters, 89, 11 (2001)

[Elmazria, 2003] O. Elmazria, M. B. Assouar, P. Renard, and P. Alnot, Physica Status Solidi (a), 196, 2, (2003)

[Engelmark, 2000] F. Engelmark, G. Fucntes, I. V. Katardjiev, A. Harsta, U. Smith, and S. Berg, The Journal of Vacuum Science and Technology A, 18, 4 (2000)

[Engelmark, 2001] F. Engelmark, G. F. Iriarte, I. V. Katardjiev, M. Ottosson, P. Muralt, and S. Berg, The Journal of Vacuum Science and Technology A, 19, 5 (2001)

[Fansler, 2008] C. Fansler, “Aluminum Nitride Thin Films,” VDM Verlag Dr. Müller (2008) 
[Farrell, 2009] R. Farrell, A. Kabulski, V. R. Pagán, S. Yeldandi, X. A. Cao, P. Famouri, J. Peter Hensel, and D. Korkakis, Materials Research Society Proceedings, Accepted (2009)

[Fujiki, 2000] M. Fujiki, M. Takahashi, S. Kikkawa, and F. Kanamaru, Journal of Material Science Letters, 19 (2000)

[Gorla, 1999] C. R. Gorla, N. W. Emanetoglu, S. Liang, W. E. Mayo, Y. Lu, M. Wraback, and H. Shen, Journal of Applied Physics, 85, 5 (1999)

[Gray, 2007] J. L. Gray and M. McLennan, “Adept,” (2007)

[Gronewold, 2007] T. M. A. Gronewold, Analytica Chimica Acta, 603 (2007)

[Gualtieri, 1994] J. G. Gualtieri, J. A. Kosinski, and A. Ballato, IEEE Transactions on Ultrasonics, Ferroelectrics, and Frequency Control, 41, 1 (1994)

[Guy, 1999] I. L. Guy, S. Muensit, and E. M. Goldys, Applied Physics Letters, 75, 26 (1999)

[Harman, 2008] J. Harman, A. Kabulski, V. R. Pagán, P. Famouri, K. R. Kasarla, L. E. Rodak, J. Peter Hensel, and D. Korakakis, The Journal of Vacuum Science and Technology $B, 26,4$ (2008)

[Hickernell, 1995] F. S. Hickernell and H. M. Liaw, Proceedings of the IEEE Ultrasonics Symposium (1995)

[Hickernell, 1996] F. S. Hickernell, Proceedings of the IEEE Ultrasonics Symposium (1996)

[Huang, 2004] C. Huang, Y. Y. Lin, and T. A. Tang, Journal of Micromechanics and Microengineering, 14 (2004)

[Hur, 2005] Y. Hur, J. Han, J. Seon, Y. E. Pak, and Y. Roh, Sensors and Actuators A, 120 (2005)

[Hwang, 2002] B.-H. Hwang, Material Science and Engineering A, 325 (2002)

[Iborra, 2004] E. Iborra, J. Olivares, M. Clement, L. Vergara, A. Sanz-Hervás, and J. Sangrador, Sensors and Actuators A, 115, (2004)

[Iborra, 2005] E. Iborra, A. Sanz-Hervás, M. Clement, L. Vergara, J. Olivares, and J. Sangrador, Proceedings of the IEEE Ultrasonics Symposium (2005)

[Iriarte, 2003] G. F. Iriarte, Journal of Applied Physics, 93, 12 (2003) 
[Iriarte, 2003] G. Iriarte, “AlN Thin Film Electroacoustic Devices,” Dissertation, ACTA Uppsala University (2003)

[Jadwisienczak, 2000] W. M. Jadwisienczak, H. J. Lozykowski, F. Perjeru, H. Chen, M. Kordesch, and I. G. Brown, Applied Physics Letters, 76, 23 (2000)

[Jaffe, 1971] B. Jaffe, W. R. Cook, Jr., and H. Jaffe, "Piezoelectric Ceramics,” Academic Press (1971)

[Jaffe, 1965] H. Jaffe and D. A. Berlincourt, Proceedings of the IEEE, 53, 10 (1965)

[Jergel, 2003] M. Jergel, C. Falcony, M. Aguilar-Frutis, M. A. Auger, O. Sanchez, and J. M. Albella, Superficies y Vacio, 16, 1 (2003)

[Jones, 1999] D. J. Jones, R. H. French, H. Müllejans, S. Loughlin, A. D. Dorneich, and P. F. Carcia, Journal of Materials Research, 14, 11 (1999)

[Kabulski, 2009] A. Kabulski, V. Pagán, and D. Korakakis, Materials Research Society Proceedings, Accepted (2009)

[Kabulski, 2009] A. Kabulski, V. Pagán, D. Cortes, R. Burda, O. M. Mukdadi, and D. Korakakis, Materials Research Society Proceedings, Accepted (2009)

[Kalantar-Zadeh, 2001] K. Kalantar-Zadeh, Y. Y. Chen, B. N. Fry, A. Trinchi, and W. Wlodarski, Proceedings of the IEEE Ultrasonics Symposium (2001)

[Kallard, 1971] T. Kallard, “Acoustic Surface Wave and Acousto-Optic Devices,” Optosonic Press (1971)

[Kao, 2002] K.-S. Kao, C.-C. Cheng, and Y.-C. Chen, IEEE Transactions on Ultrasonics, Ferroelectrics, and Frequency Control, 49, 3 (2002)

[Kar, 2004] J. P. Kar, G. Bose, and S. Tuli, Surface and Coatings Technology, 198 (2005)

[Koch, 1996] M. Koch, A. G. R. Evans, and A. Brunnschweiler, Colloquim on Microengineered Components for Fluids (1996)

[Krautkrämer, 1990] J. Krautkrämer and H. Krautkrämer, "Ultrasonic Testing of Materials, $4^{\text {th }}$ Edition," Springer-Verlag (1990)

[Länge, 2008] K. Länge, B. E. Rapp, and M. Rapp, Analytical and Bioanalytical Chemistry, 391 (2008)

[Lappalainen, 1999] J. Lappalainen, J. Frantti, and V. Lantto, Journal of the American Ceramic Society, 82, 4 (1999) 
[Lee, 2005] Y. S. Lee, D. S. Yoon, and T. S. Kim, Integrated Ferroelectrics, 69 (2005)

[Leung, 2003] T. T. Leung and C. W. Ong, Integrated Ferroelectrics, 57 (2003)

[Liufu, 1998] D. Liufu and K.C. Cao, The Journal of Vacuum Science and Technology A, 16, 4 (1998)

[MacKenzie, 1996] J. D. MacKenzie, C. R. Abernathy, S. J. Pearton, U. Hömmerich, X. Wu, R. N. Schwartz, R. G. Wilson, and J. M. Zavada, Applied Physics Letters, 69, 14 (1996)

[Mahmood, 2003] A. Mahmood, R. Machorro, S. Muhl, J. Heiras, F. F. Castillón M. H. Farías, and E. Andrade, Diamond and Related Materials, 12 (2003)

[Martin, 2004] F. Martin, P. Muralt, M.-A. Dubois, and A. Pezous, The Journal of Vacuum Science and Technology A, 22, 2 (2004)

[McGuire, 1973] G. E. McGuire, G. K. Schweitzer, and T. A. Carlson, Inorganic Chemistry, 12, 10 (1973)

[McNeil, 1993] L. E. McNeil, M. Grimsditch, and R. H. French, Journal of the American Ceramic Society, 76, 5 (1993)

[Meng, 1994] W. J. Meng, “Properties of Group III Nitrides,” INSPEC, Ed. J. H. Edgar (1994)

[Mortet, 2002] V. Mortet, O. Elmazria, M. Nesladek, M. B. Assouar, G. Vanhoyland, J. D’Haen, M. D'Olieslaeger, and P. Alnot, Applied Physics Letters, 81, 9 (2002)

[Moulder, 1995] J. F. Moulder, W. F. Stickle, P. E. Sobol, and K. D. Bomben, "Handbook of X-ray Photoelectron Spectroscopy,” Eds. J. Chastain and R. C. King, Jr., ULVAC-PHI, Inc. (1995)

[Muhl, 1997] S. Muhl, J. A. Zapient, J. M. Mendez, and E. Andrade, Journal of Physics D: Applied Physics, 30, 15 (1997)

[Muller, 2003] R. S. Muller and T. I. Kamins, "Device Electronics for Integrated Circuits, Third Edition," John Wiley \& Sons (2003)

[Muralt, 1997] P. Muralt, Integrated Ferroelectrics, 17 (1997)

[Nanoscience Instruments, 2008] NanoScience Instruments, "Atomic Force Microscopy," Accessed April 16, 2009 (2008)

$<$ http://www.nanoscience.com/education/AFM.html $>$ 
[Nakahata, 1995] H. Nakahata, K. Higaki, S. Fujii, A. Hachigo, H. Kitabayashi, K. Tanabe, Y. Seki, and S. Shikata, Proceedings of the IEEE Ultrasonics Symposium (1995)

[Olivares, 2005] J. Olivares, M. Clement, E. Iborra, L. Vergara, J. L. Sánchez-Rojas, J. Vázquez, and P. Sanz, Smart Sensors, Actuators, and MEMS II, 5836 (2005)

[Pagán, 2009] V. R. Pagán and D. Korakakis, Materials Research Society Proceedings, Accepted (2009)

[Park, 2004] J. Y. Park, H. C. Lee, and J. U. Bu, $34^{\text {th }}$ European Microwave Conference (2004)

[Pashley, 1964] D. W. Pashley, M. J. Stowell, M. H. Jacobs, and T. J. Law, Advances in Physics, 10, 103 (1964)

[Pearce, 1981] L.G. Pearce, R. L. Gunshor, and R. F. Pierret, Proceedings of the IEEE Ultrasonics Symposium (1981)

[Porowski, 2002] S. Porowski, I. Grzegory, D. Kolesnikov, W. Lojkowski, V. Jager, W. Jager, V. Bogdanov, T. Suski, and S. Krukowski, Journal of Physics: Condensed Matter, 14 (2002)

[Prume, 2004] K. Prume, P. Gerber, C. Kügeler, A. Roelofs, U. Böttger, R. Waser, T. Schmitz-Kempen, and S. Tiedke, IEEE International Ultrasonics, Ferroelectrics, and Frequency Control Joint $50^{\text {th }}$ Anniversary Conference (2004)

[Shannigrahi, 2004] S. R. Shannigrahi, F. E. H. Tay, K. Yao, R. N. P. Choudhary, Journal of the European Ceramic Society, 24 (2004)

[Sharma, 2003] R. K. Sharma, J. Ning, F. Hanhua, and R. Gopalakrishman, Materials Research Society Symposium Proceedings (2003)

[Shibata, 1995] Y. Shibata, K. Kaya, K. Akashi, M. Kanai, T. Kawai, and S. Kawai, Journal of Applied Physics, 77, 4 (1995)

[Shimada, 1998] K. Shimada, T. Sota, and K. Suzuki, Journal of Applied Physics, 84, 9 (1998)

[Smits, 1991] J. G. Smits and W.-S. Choi, IEEE Transaction on Ultrasonics, Ferroelectrics, and Frequency Control, 38, 3 (1991)

[Thornton, 1974] J. A. Thornton, The Journal of Vacuum Science and Technology, 11, 4 (1974) 
[Thornton, 1977] J. A. Thornton, Annual Reviews, 7 (1977)

[Tonisch, 2006] K. Tonisch, V. Cimalla, Ch. Foerster, D. Dontsov, and O. Ambacher, Physica Status Solidi (c), 3, 6 (2006)

[Tonisch, 2006] K. Tonisch, V. Cimalla, Ch. Foerster, H. Romanus, O. Ambacher, and D. Dontsov, Sensors and Actuators A, 132 (2006)

[Trolier-McKinstry, 2004] S. Trolier-McKinstry and P. Muralt, Journal of Electroceramics, 12 (2004)

[Tsubouchi, 1981] K. Tsubouchi, K. Sugai, and N. Mikoshiba, Proceedings of the IEEE Ultrasonics Symposium (1981)

[Tsubouchi, 1985] K. Tsubouchi and N. Mikoshiba, IEEE Transactions on Sonics and Ultrasonics, SU-32, 5 (1985)

[Uehara, 2005] K. Uehara, Y. Aota, T. Shibata, S. Kameda, H. Nakase, Y. Isota, and K. Tsubouchi, Japanese Journal of Applied Physics, 44, 6B (2005)

[Walraven, 2003] J. A. Walraven, ITC International Test Conference (2003)

[Wang, 2005] L.-P. Wang, D. S. Shim, Q. Ma, V. R. Rao, E. Ginsburg, and A. Talalyevsky, The Journal of Vacuum Science and Technology A, 23, 4 (2005)

[Watanabe, 2000] W. Watanabe, Y. Hara, T. Tokuda, N. Kitazawa, and Y. Nakamura, Surface Engineering, 16, 3 (2000)

[Welch, 1996] W. Welsch, C. Klein, M. von Schickfus, and S. Hunklinger, Analytical Chemistry, 68, 13 (1996)

[Wilson, 1994] R. G. Wilson, R. N. Schwartz, C. R. Abernathy, S. J. Pearton, N. Newman, M. Rubin, T. Fu, and J. M. Zavada, Applied Physics Letters., 65, 8 (1994)

[Woollam, 2000] J. A. Woollam, J. N. Hilfiker, T. E. Tiwald, C. L. Bungay, R. A. Synowicki, D. E. Meyer, C. M. Herzinger, G. L. Pfeiffer, G. T. Cooney, and S. E. Green, SPIE Proceedings (2000)

[Woollam, 2001] J. A. Woollam Co., Inc., "A Short Course in Ellipsometry,” (2001)

[Xu, 2002] F. Xu, R. A. Wolf, T. Yoshimura, and S. Trolier-McKinstry, $11^{\text {th }}$ International Symposium on Electrets (2002)

[Yao, 2004] K. Yao, S. Shannigrahi, and F. E. H. Tay, Sensors and Actuators A, 112 (2004) 
[Yu, 2005] Y. Yu, T.-L. Ren, and L.-T. Liu, Integrated Ferroelectrics, 69 (2005)

[Zaitsev, 2001] A. M. Zaitsev, "Optical Properties of Diamond: A Data Handbook," Springer (2001)

[Zhao, 2009] J. L. Zhao, S. T. Tan, S. Iwan, X. W. Sun, W. Liu, and S. J. Chua, Applied Physics Letters, 94, 093506 (2009)

[Zhang, 2003] Q. Q. Zhang, S. J. Gross, S. Tadigadapa, T. N. Jackson, F. T. Djuth, and S. Trolier-McKinstry, Sensors and Actuators A, 105, 1 (2003) 


\section{APPENDIX A: LIST OF ACRONYMS}

$\begin{array}{lll}\text { AES } & : & \text { Auger Electron Spectroscopy } \\ \text { AFM } & : & \text { Atomic Force Microscopy } \\ \text { AT } & : & \text { Attenuation Length } \\ \text { BAW } & : & \text { Bulk Acoustic Wave } \\ \text { DC-RMS } & : & \text { DC Reactive Magnetron Sputtering } \\ \text { DUT } & : & \text { Device Under Test } \\ \text { ED } & : & \text { Escape Depth } \\ \text { EDAX } & : & \text { Energy Dispersive Analysis of X-rays } \\ \text { EDXS } & : & \text { Energy Dispersive X-ray Spectroscopy } \\ \text { ESCA } & : & \text { Electron Spectroscopy for Chemical Analysis } \\ \text { FTIR } & : & \text { Fourier Transform Infrared Spectroscopy } \\ \text { FWHM } & : & \text { Full Width Half Maximum } \\ \text { IMFP } & : & \text { Inelastic Mean Free Path } \\ \text { ID } & : & \text { Information Depth } \\ \text { IDT } & : & \text { Interdigitated or Interdigital Transducer } \\ \text { MEMS } & : & \text { Microelectromechanical System } \\ \text { MIM } & : & \text { Metal-Insulator-Metal } \\ \text { MIS } & : & \text { Metal-Insulator-Semiconductor } \\ \text { PL } & : & \text { Photoluminescence } \\ \text { PNA } & : & \text { Portable Network Analyzer } \\ \text { RSAW } & : & \text { "Rayleigh-type” SAW } \\ \text { SAW } & : & \text { Surface Acoustic Wave } \\ \text { SEM } & : & \text { Scanning Electron Microscopy } \\ \text { SCCM } & : & \text { Standard Cubic Centimeters per Minute } \\ \text { SIMS } & : & \text { Secondary Ion Mass Spectrometry } \\ \text { SIS } & : & \text { Semiconductor-Insulator-Semiconductor } \\ \text { SMR } & : & \text { Solidly Mounted Resonator } \\ \text { SNR } & : & \text { Signal-to-Noise Ratio } \\ \text { TCD } & : & \text { Temperature Coefficient of Delay Time } \\ \text { TCF } & : \text { Temperature Coefficient of Frequency } \\ \text { TFBAR } & : \text { Thin-Film Bulk Acoustic Resonator } \\ \text { UNCD } & : & \text { Ultrananocrystalline Diamond } \\ \text { UV } & : & \text { Ultraviolet } \\ \text { XPS } & : & \text { X-Ray Photoelectron Spectroscopy } \\ \text { XRD } & : & \text { X-Ray Diffraction } \\ & & \end{array}$


APPENDIX B: PHOTOMASKS

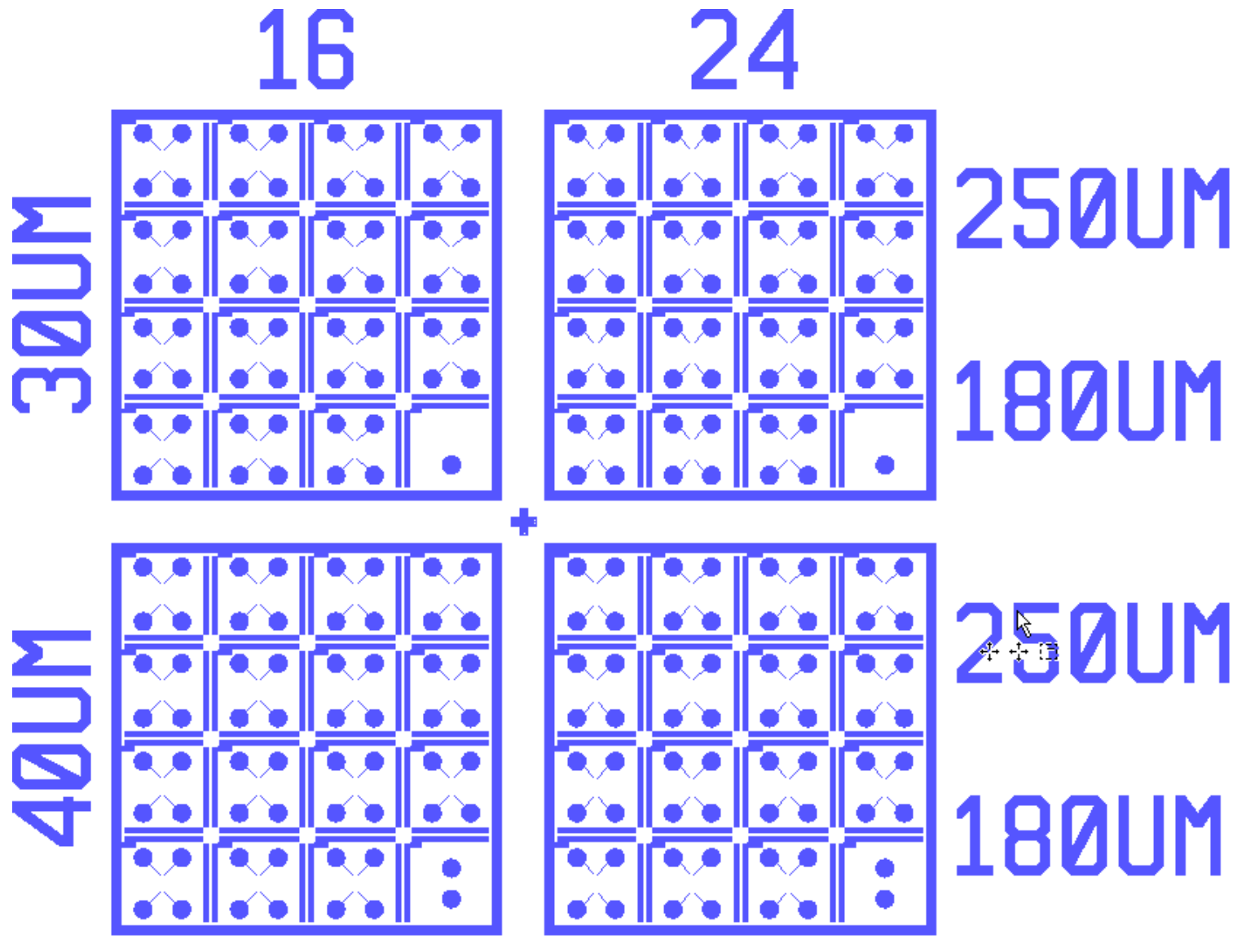

Figure B.1. Low frequency interdigitated transducer mask.



Figure B.2. Low frequency interdigitated transducer mask (zoomed). 


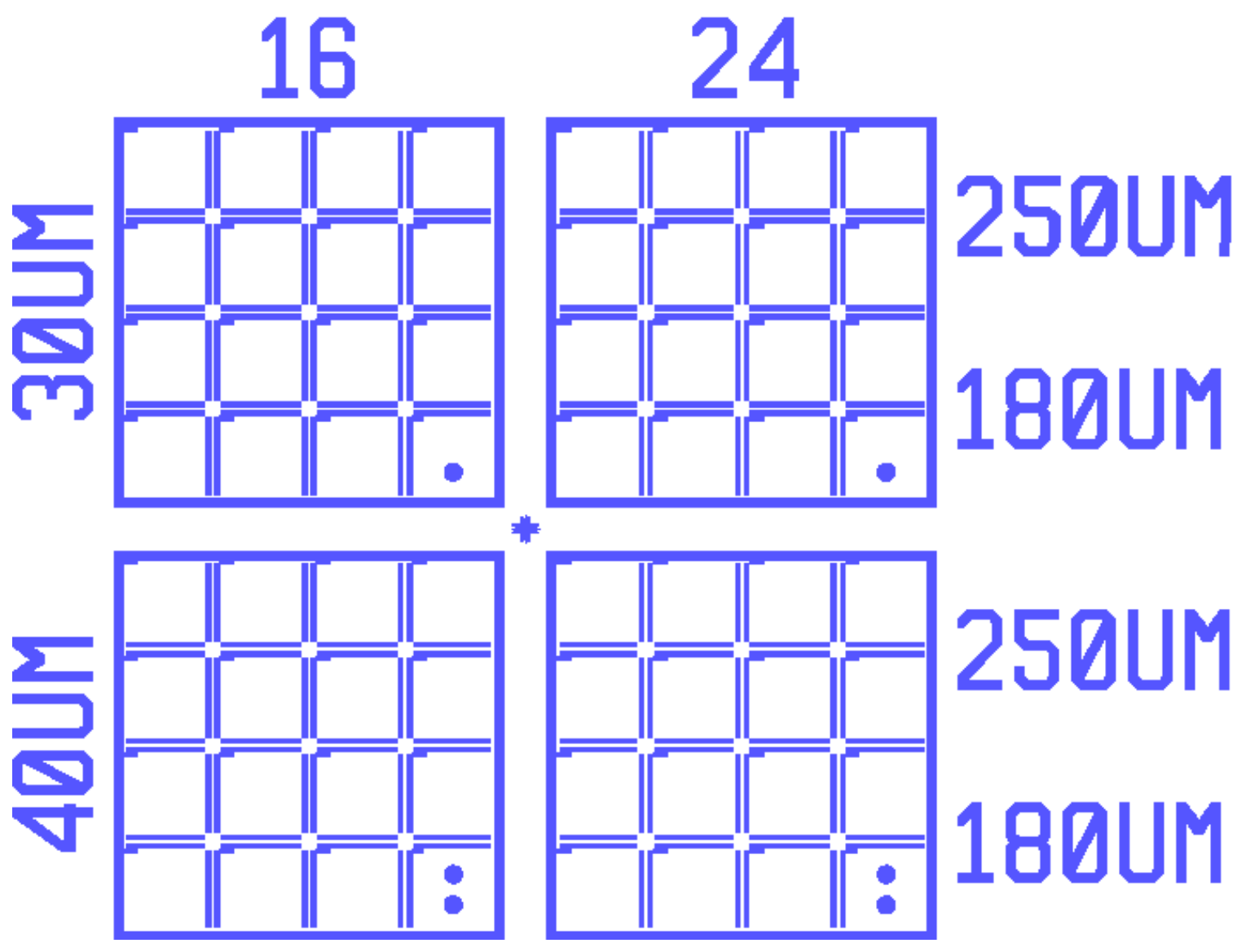

Figure B.3. High frequency interdigitated transducer mask.
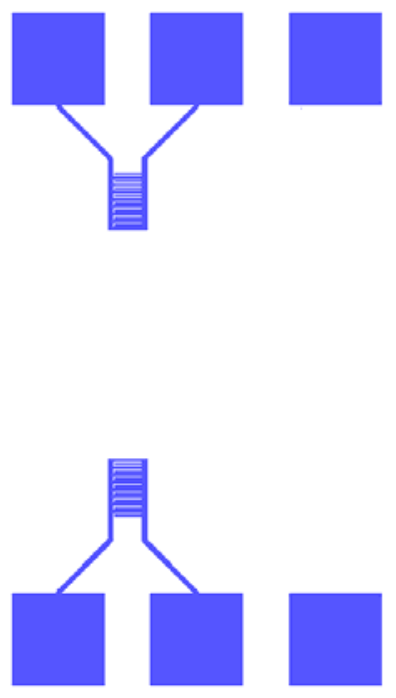

Figure B.4. High frequency interdigitated transducer mask (zoomed). 


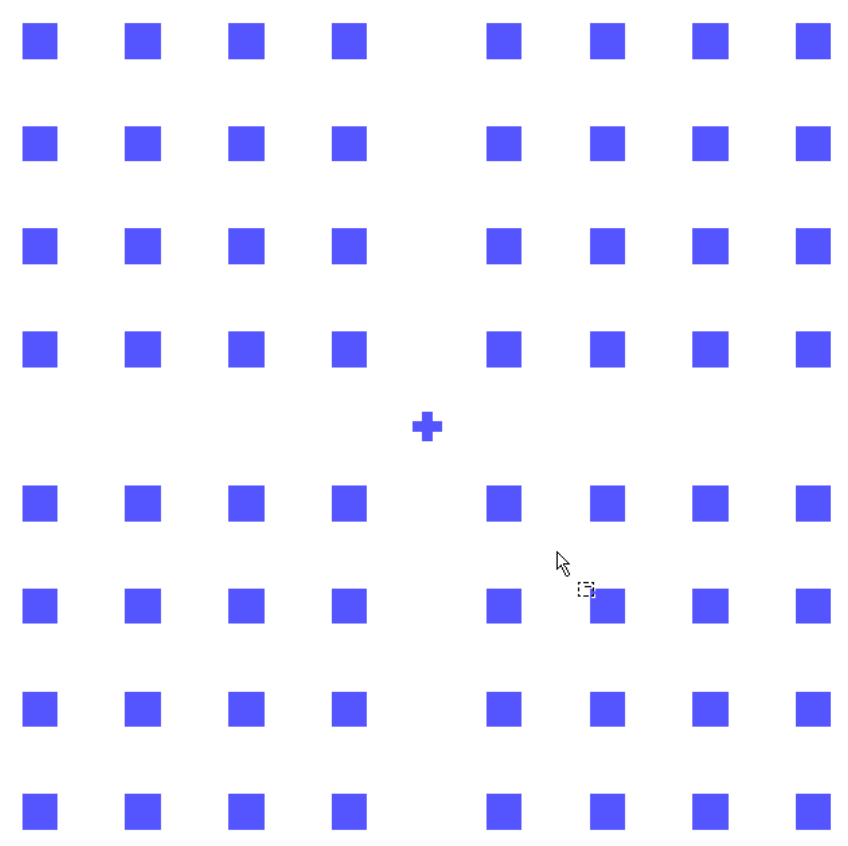

Figure B.5. Diaphragm mask. 


\section{HigH FREQUENCY SETUP}

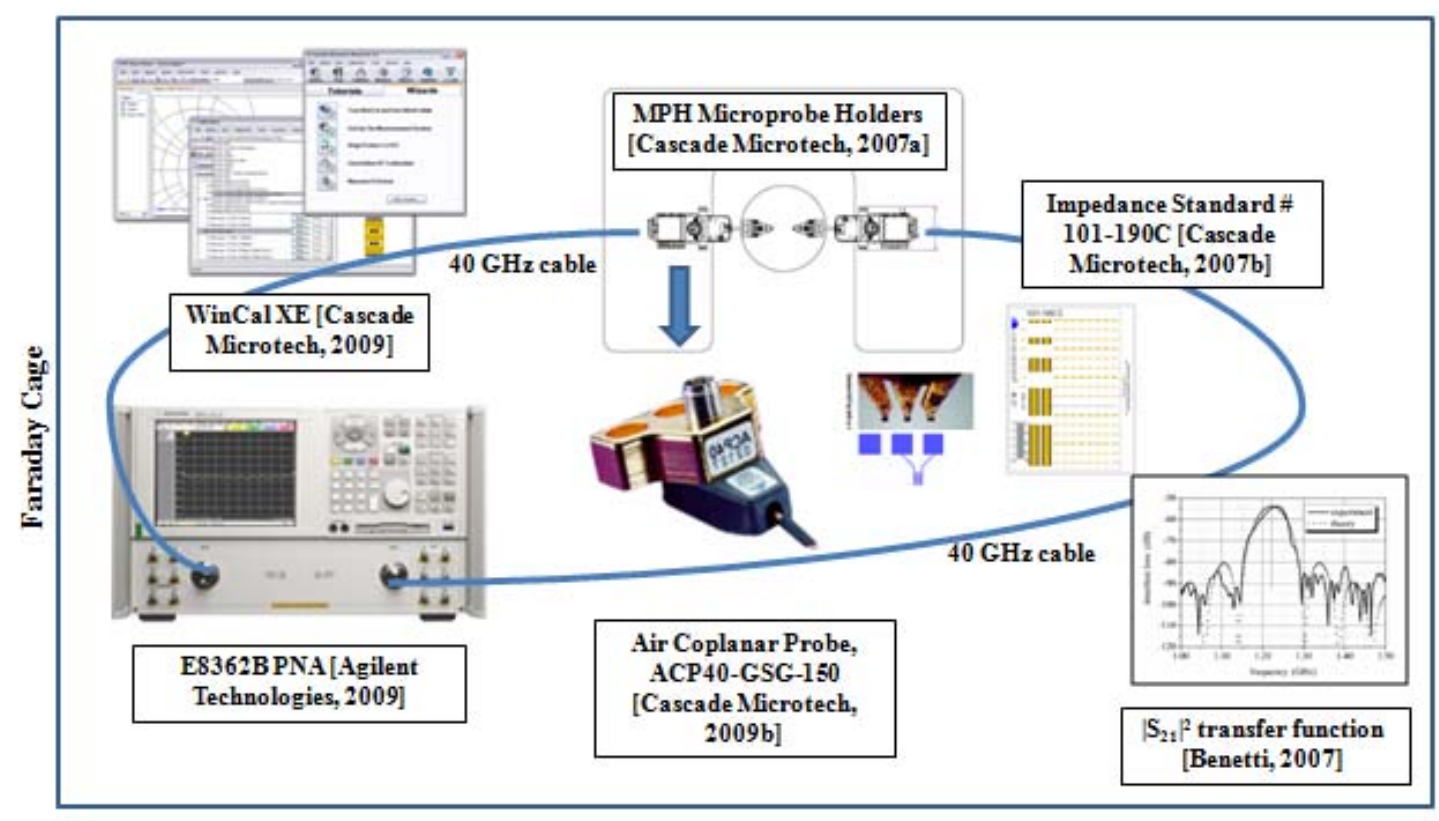

HF setup should provide the capability of measuring the SAW platforms up to $20 \mathrm{GHz}$.

\begin{tabular}{|c|l|}
\hline Quantity & Description \\
\hline 2 & ACP40-GSG-150, 40GHz probe, GSG, BeCu \\
\hline 1 & $\begin{array}{l}\text { MPHM-L, Micro probe holder, magnetic base } \\
\text { Left position }\end{array}$ \\
\hline 1 & $\begin{array}{l}\text { MPHM-R, Micro probe holder, magnetic base } \\
\text { Right position }\end{array}$ \\
\hline 1 & $101-190$, LRM impedance standard, GSG \\
\hline 2 & $101-162 \mathrm{~B}$, 40GHz flexible coax cable, $\mathrm{K}$ connectors \\
\hline 1 & $142-171$, WinCal XE software \\
\hline
\end{tabular}




\section{APPENDIX D: MATLAB CODE}

$\%$ Matlab written by Vincent R. Pagan

\% Copyright 2009

\% Material properties of AlN from [Tsubouchi, 1981]

$\%$ Stiffness coefficients

$\mathrm{c} 11=345 \mathrm{E} 9$

$\mathrm{c} 12=125 \mathrm{E} 9$

$\mathrm{c} 13=120 \mathrm{E} 9$

$\mathrm{c} 33=395 \mathrm{E} 9$

$\mathrm{c} 44=118 \mathrm{E} 9 ;$

$\mathrm{c} 66=110 \mathrm{E} 9 ;$

$\%$ Stiffness matrix for $6 \mathrm{~mm}$ crystal class


c44 $0 ; 0 \begin{array}{lllllll}0 & 0 & 0 & 0 & 0 & 2 *(\mathrm{c} 11-\mathrm{c} 12)]\end{array}$

$\%$ Compliance matrix

$\mathrm{S}=\mathrm{C}^{\wedge}-1$

$\%$ Piezoelectric stress constants

$\mathrm{e} 15=-0.48$

$\mathrm{e} 31=-0.58$

$\mathrm{e} 33=1.55$;

$\%$ Piezoelectric matrix

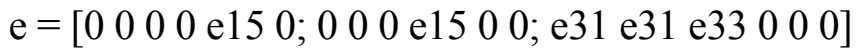

$\%$ Piezoelectric strain constants

$\mathrm{d}=\mathrm{e}^{*} \mathrm{~S}^{\prime}$

$\%$ Output results: $d 33$ and s33,E

$\mathrm{d} 33=\mathrm{d}(3,3) * 10^{\wedge} 12$

fprintf('pm/V\n')

$\mathrm{s} 33 \mathrm{E}=\mathrm{S}(3,3)$

fprintf('Pa^-1\n') 
APPENDIX E: LASER ABLATION CODE

;PROGRAM ABLATE_CANTILEVERS

; this program was written to cut three edges out of a square diaphragm

DVAR \$ZSTART ;set starting z position

DVAR SDISTX ;set x position

DVAR \$DISTY ;set y position

DVAR \$DISTZ ;set z position

DVAR \$SPEED ; ;set drill speed

DVAR \$NUMPASS ; set number of passes

\$ZSTART $=81.250 \quad$;set when DOF is found

$\$ S P E E D=5 \quad$;set the speed to $5 \mathrm{~mm} / \mathrm{s}$

\$NUMPASS $=1 \quad$;set the number of passes to 1

SDISTX $=0.01 \quad$;set the $\mathrm{x}$ dist to $10 \mathrm{um}$

$\$ D I S T Y=0.01 \quad$;set the y dist to $10 \mathrm{um}$

$\$ D I S T Z=0 \quad$;set the $\mathrm{z}$ dist to 0

ENABLE X Y Z

G90

G71

G92 X0 Y0

REPEAT \$NUMPASS

OPENSHUTTER

G1 X+\$DISTX Y-0 Z-0 F\$SPEED

G1 X-0 Y-\$DISTY Z-0 F\$SPEED

G1 X-\$DISTX Y-0 Z-0 F\$SPEED

CLOSESHUTTER

G1 X-0 Y+\$DISTY Z-0 F\$SPEED

ENDREPEAT

M2 


\section{APPENDIX F: ADEPT INFILE}

*TITLE aln with pt contacts

mesh $n x=250$, xres $=1.0$

bc $\mathrm{mbc}=2$,wdfrt $=1.6, \mathrm{wdbck}=1.6$

*LAYER silicon substrate

layer $\mathrm{tm}=3.5, \mathrm{na}=5.8513 \mathrm{e} 16, \mathrm{eg}=1.12, \mathrm{nc}=3.2216 \mathrm{e} 19$

$+\mathrm{nv}=1.8187 \mathrm{e} 19, \mathrm{ks}=11.7, \mathrm{chi}=4.05$, un $=1350$., up $=480$.

+ eaa $=0.045$,taup. shr $=1.0 \mathrm{e}-9$,taun.. hr $=1.0 \mathrm{e}-9$

+ et.shr $=0.0, \mathrm{vsatn}=1.0 \mathrm{e} 7, \mathrm{vsatp}=1.0 \mathrm{e} 7$

*LAYER aln thin film

layer $\mathrm{tm}=0.7, \mathrm{na}=0.0, \mathrm{eg}=6.2, \mathrm{nc}=6.2354 \mathrm{e} 18$

$+\mathrm{nv}=4.8844 \mathrm{e} 20, \mathrm{ks}=8.5, \mathrm{chi}=0.6, \mathrm{un}=300 . \mathrm{up}=14$.

+ ead $=0.17$,taup. shr $=1.0 \mathrm{e}-9$,taun. shr $=1.0 \mathrm{e}-9$

+ et.shr $=0.0, \mathrm{vsatn}=1.0 \mathrm{e} 7, \mathrm{vsatp}=1.0 \mathrm{e} 7$

bc $\mathrm{mbc}=2, \mathrm{wdfrt}=5.05, \mathrm{wdbck}=5.05$

solve itmax $=100$

$\mathrm{i}-\mathrm{v}$ vstart $=-5.0, \mathrm{vstop}=0.0, \mathrm{dv}=1.0$

output info $=5$,step $=1$ 


\section{APPENDIX G: SAMPLE ANSYS BATCH FILE}

!/batch,list

! $* * * * * * * * * * * * * * * * * * * * * * * * * * * * * * * * * * * * * * * * * * * * * * * * * * * * * * *$

! Copyright 2008, Vincent Paga'n

! Last updated June 11, 2008

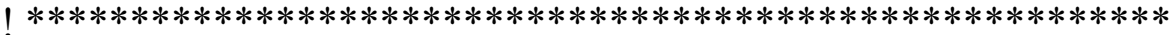

/title,Analysis of a Piezoelectric Bimorph Beam

/nopr

/com,

/PREP7

! **********************************************************

! SIMULATION PARAMETERS - PLEASE MAKE MODIFICATIONS

HERE!!!

! 3" wafer

radius $=1.5$

thickness $=50 \mathrm{e}-6$

! Specify the thickness of each film

! For silicon, the height is the thickness / 2

Hsi=thickness $/ 2$

Hsi3n4=400e-9

Haln $=1$ e- 6

! Specify applied DC voltage

$\mathrm{V}=50$

!length

$\mathrm{L}=1.4142 *$ radius $* 25.4 \mathrm{e}-3$

! Calculate simulation clamped length

$\mathrm{Lc}=\mathrm{L} / 3$

! Set model parameters (used in forming areas)

Ldivsi $=10$

Ldivaln $=40$

Ldivsi3n4=30

! Set mesh parameters (used to refine mesh)

mesher $=10$ 


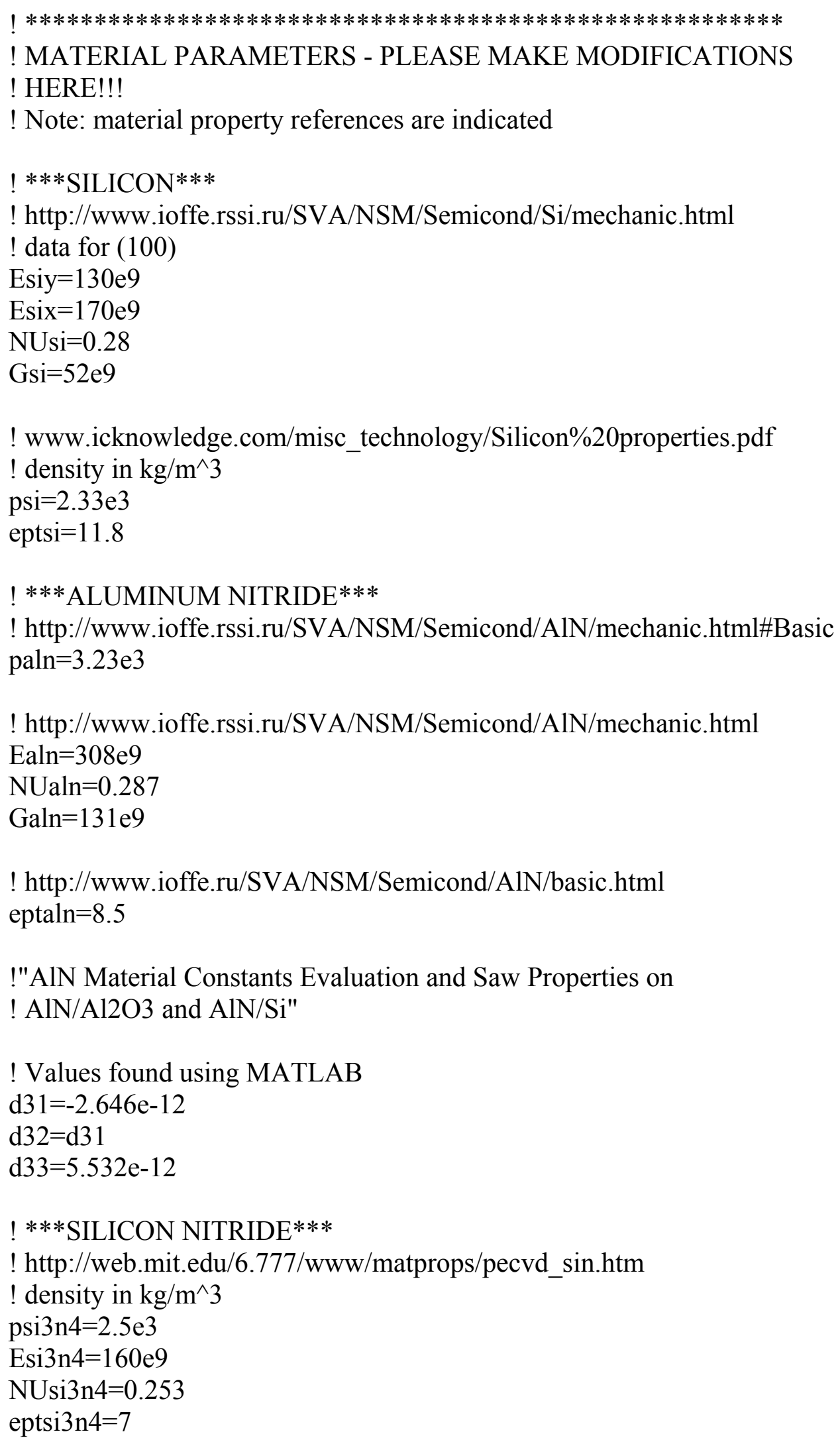

!"AlN Material Constants Evaluation and Saw Properties on ! AlN/A12O3 and AlN/Si"

! Values found using MATLAB

$\mathrm{d} 31=-2.646 \mathrm{e}-12$

$\mathrm{d} 32=\mathrm{d} 31$

$\mathrm{d} 33=5.532 \mathrm{e}-12$

!***SILICON NITRIDE***

! http://web.mit.edu/6.777/www/matprops/pecvd_sin.htm

! density in $\mathrm{kg} / \mathrm{m}^{\wedge} 3$

psi3n $4=2.5 \mathrm{e} 3$

Esi3n4=160e9

NUsi3n $4=0.253$

eptsi3n $4=7$ 


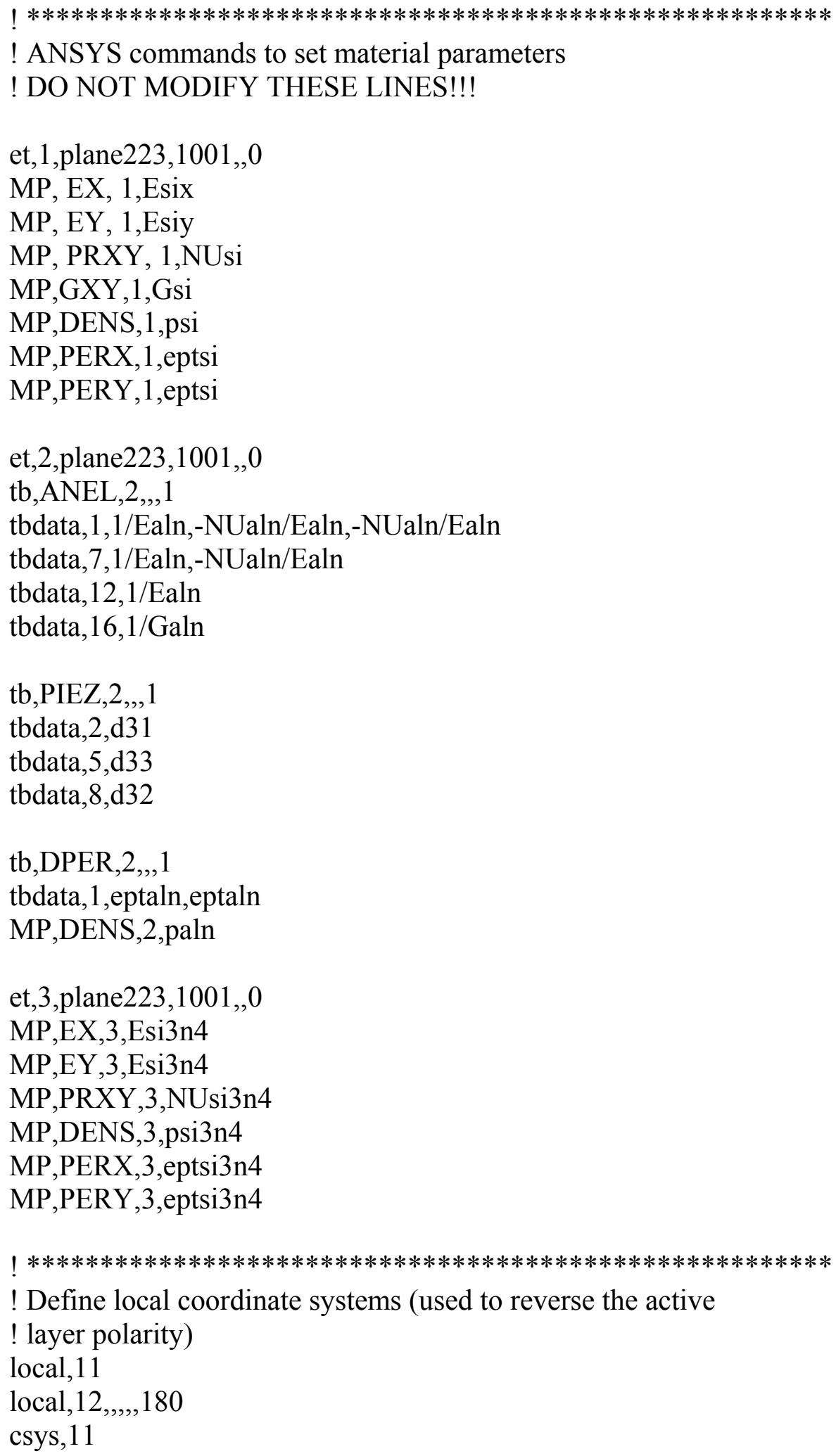




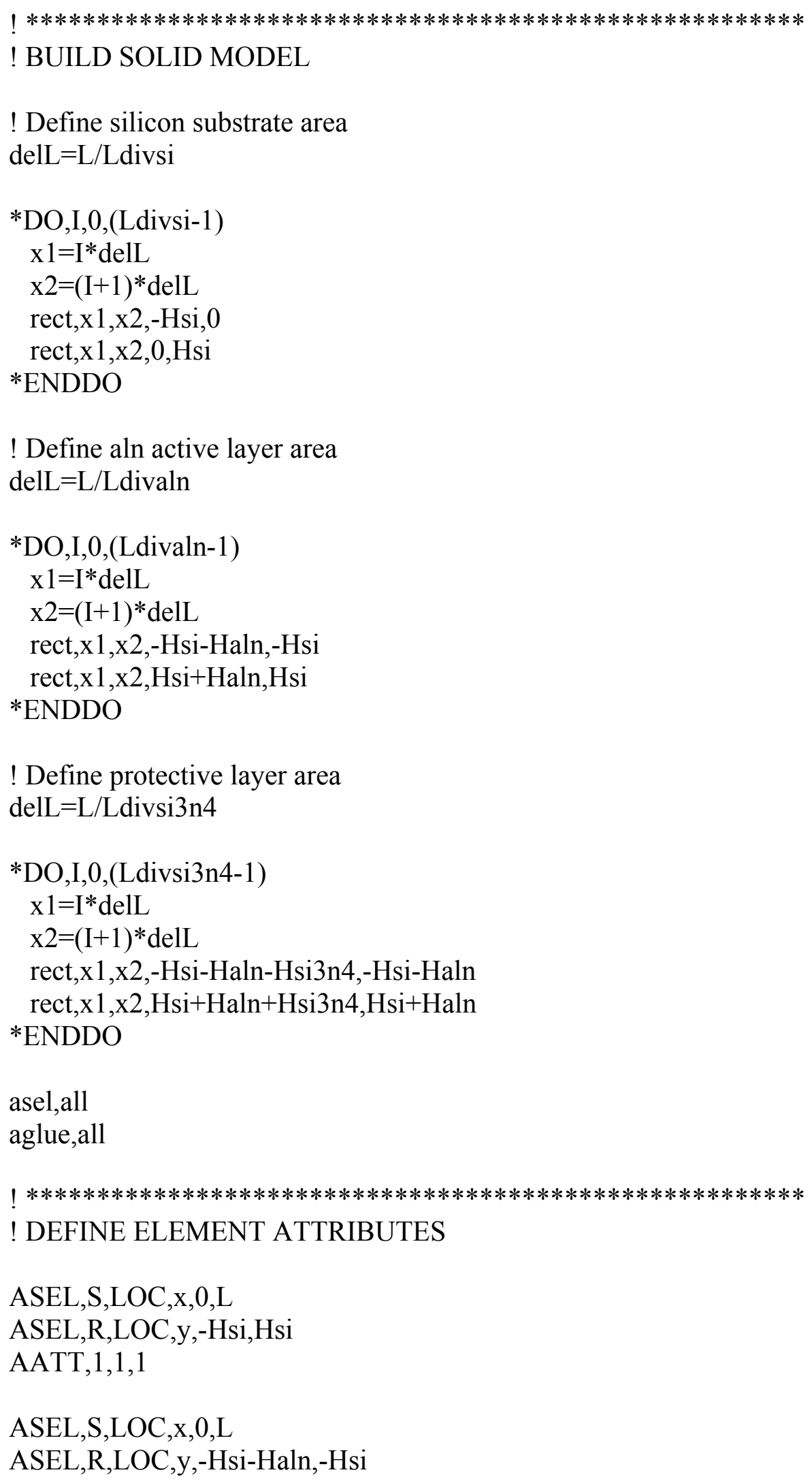


AATT,2,2,2

ASEL,S,LOC,x,0,L

ASEL,R,LOC,y,Hsi,Hsi+Haln

AATT, $2,2,2$

ASEL,S,LOC,X,0,L

ASEL,R,LOC,y,-Hsi-Haln-Hsi3n4,-Hsi-Haln

AATT, 3,3,3

ASEL,S,LOC,x,0,L

ASEL,R,LOC,y,Hsi+Haln,Hsi+Haln+Hsi3n4

AATT, 3,3,3

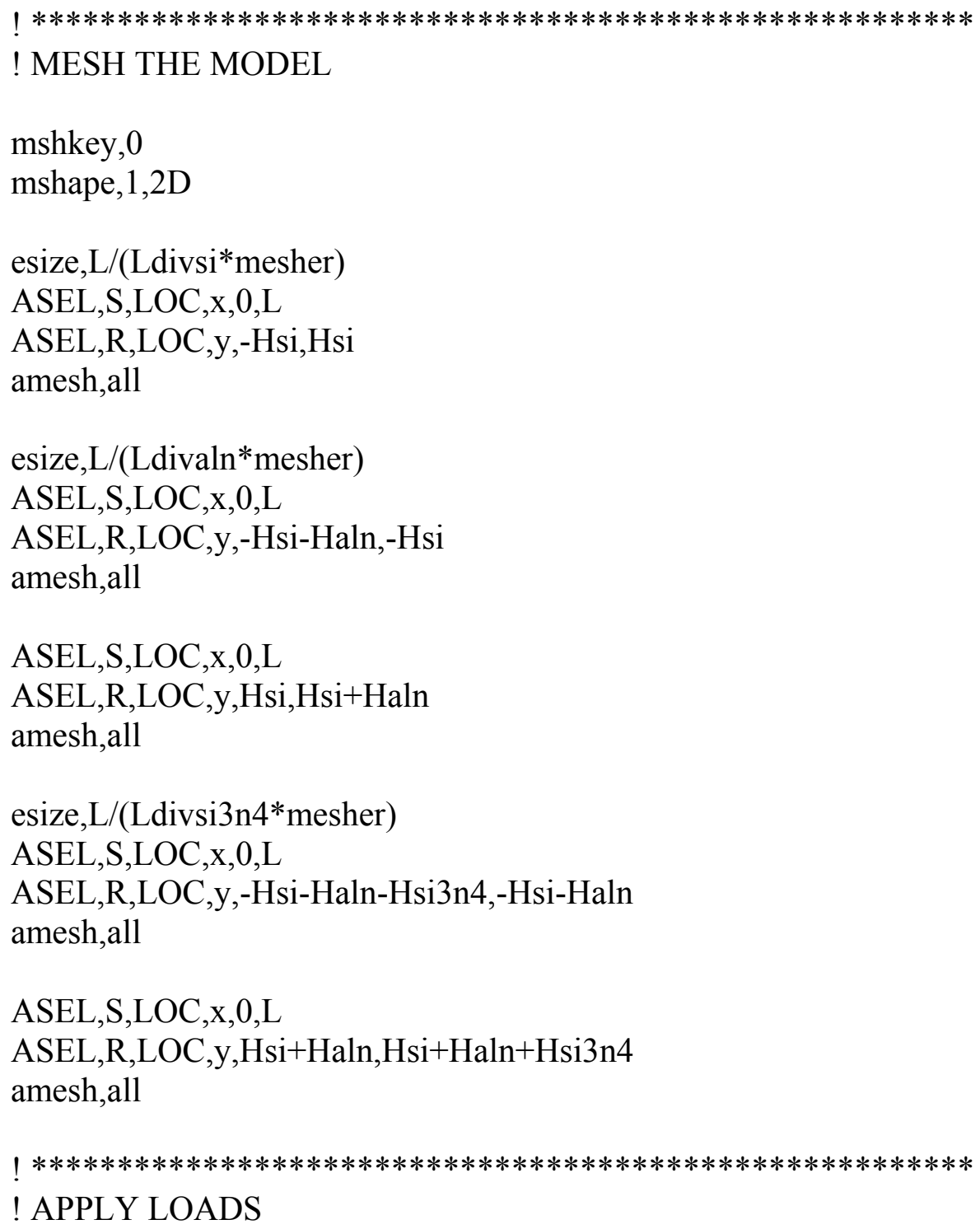




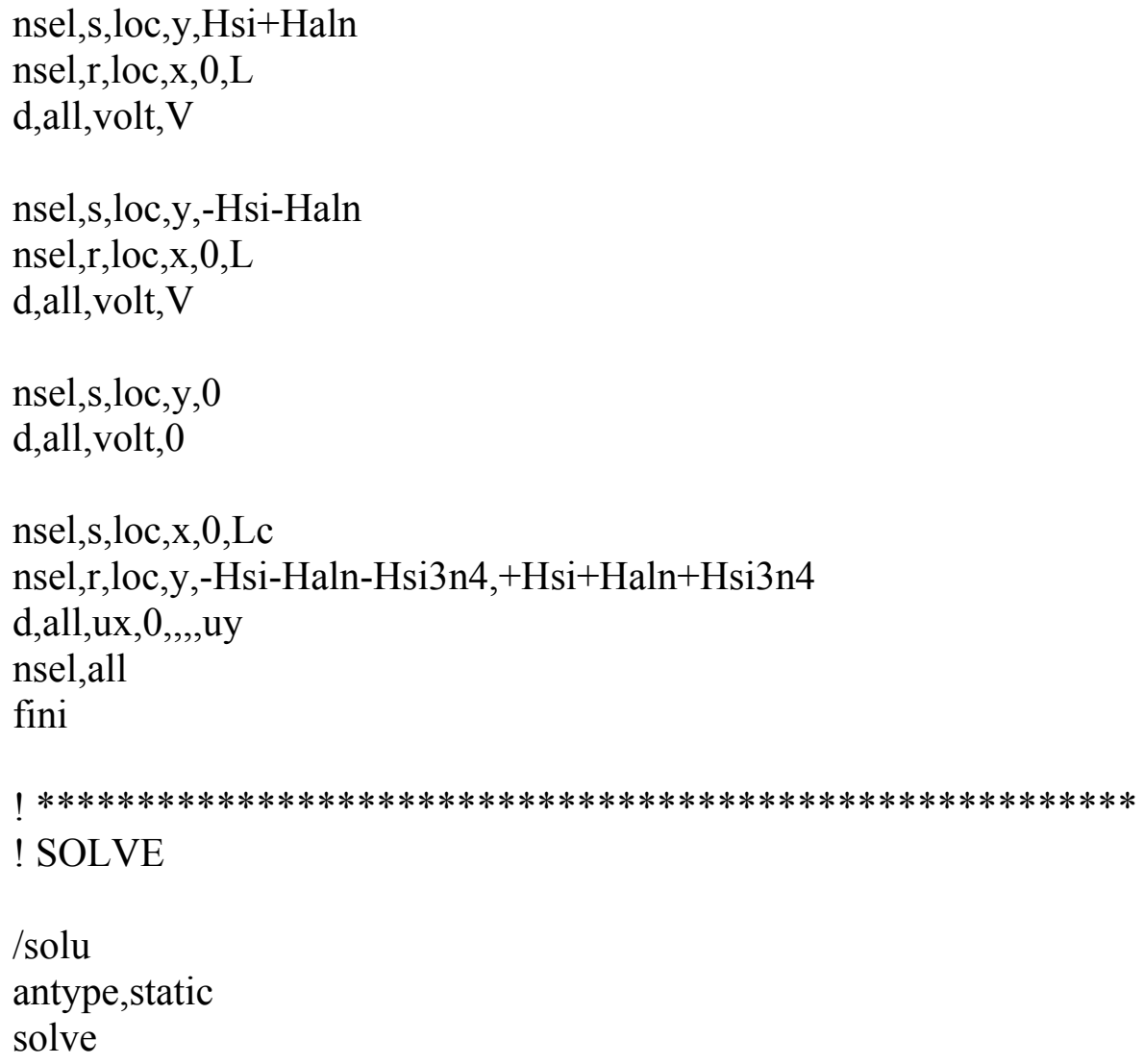

